\title{
Studies of the Use of Electrochemical Impedance Spectroscopy to Characterize and Assess the Performance of Lacquers Used to Protect Aluminum Sheet and Can Ends
}

\author{
by
}

\section{Mohammad Ali}

A thesis submitted to the Faculty of Graduate and Postdoctoral Affairs in partial fulfillment of the requirements for the degree of

\author{
Master of Applied Science \\ in \\ Department of Mechanical and Aerospace Engineering \\ Carleton University \\ Ottawa, Ontario
}

The Master of Applied Science in Materials Engineering is a joint program with the University of Ottawa, administered by Ottawa-Carleton Institute for Mechanical and Aerospace Engineering

(C) 2013

Mohammad Ali 
Library and Archives

Canada

Published Heritage

Branch

395 Wellington Street

Ottawa ON K1A ON4

Canada
Bibliothèque et

Archives Canada

Direction du

Patrimoine de l'édition

395 , rue Wellington

Ottawa ON K1A ON4

Canada
Your file Votre référence

ISBN: $978-0-494-94255-0$

Our file Notre référence

ISBN: $978-0-494-94255-0$
NOTICE:

The author has granted a nonexclusive license allowing Library and Archives Canada to reproduce, publish, archive, preserve, conserve, communicate to the public by telecommunication or on the Internet, loan, distrbute and sell theses worldwide, for commercial or noncommercial purposes, in microform, paper, electronic and/or any other formats.

The author retains copyright ownership and moral rights in this thesis. Neither the thesis nor substantial extracts from it may be printed or otherwise reproduced without the author's permission.
AVIS:

L'auteur a accordé une licence non exclusive permettant à la Bibliothèque et Archives Canada de reproduire, publier, archiver, sauvegarder, conserver, transmettre au public par télécommunication ou par l'Internet, prêter, distribuer et vendre des thèses partout dans le monde, à des fins commerciales ou autres, sur support microforme, papier, électronique et/ou autres formats.

L'auteur conserve la propriété du droit d'auteur et des droits moraux qui protege cette thèse. $\mathrm{Ni}$ la thèse ni des extraits substantiels de celle-ci ne doivent être imprimés ou autrement reproduits sans son autorisation.
In compliance with the Canadian Privacy Act some supporting forms may have been removed from this thesis.

While these forms may be included in the document page count, their removal does not represent any loss of content from the thesis.
Conformément à la loi canadienne sur la protection de la vie privée, quelques formulaires secondaires ont été enlevés de cette thèse.

Bien que ces formulaires aient inclus dans la pagination, il n'y aura aucun contenu manquant. 


\begin{abstract}
This study involved investigating the feasibility of using Electrochemical Impedance Spectroscopy to assess the performance of coatings used to protect aluminum in beverage containers, and developing an accelerated testing procedure. In the preliminary investigation, tests were performed to ensure that the EIS systems at hand are capable, functional and consistent. This was followed by EIS testing of kitchen-aluminum foil and high-impedance epoxy polymer as a baseline for chemically-active and chemically-inert systems. The ability of EIS to differentiate between intact and flawed coatings was tested by investigating deliberately damaged coatings. The effects of varying the $\mathrm{pH}$ and oxygen content on the performance of the coated aluminum samples were also tested. From this investigation, it has been concluded that EIS can be used to differentiate between intact and flawed coatings and detect corrosion before it is visually observable. Signatures of corrosion have been recorded and a preliminary testing procedure has been drawn.
\end{abstract}




\section{Acknowledgements}

I would like to extend my deepest gratitude to my thesis supervisor Professor Glenn McRae and Dr. Dave McCracken for their advice, guidance, support and patience during the difficult process of carrying out my research. I could not have completed this work without them.

I would like to thank Dr. John Hunter of Novelis.

I would like to thank my mother, father, sister, brother and my fiancé for their constant encouragement and moral support.

I would also like to thank all of my friends for helping me through all the tough times.

Finally, I would like to express my deep appreciation for NSERC for their financial support, without which this research would not be possible. 


\section{Table of Contents}

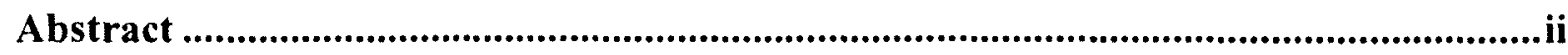

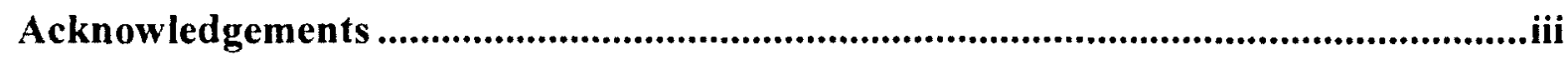

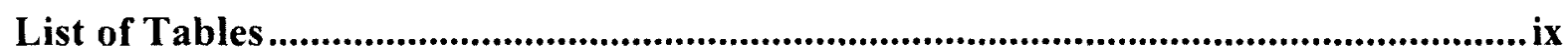

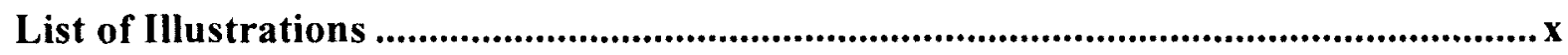

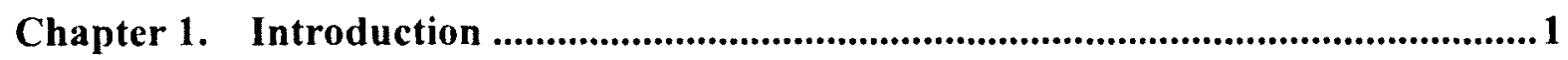

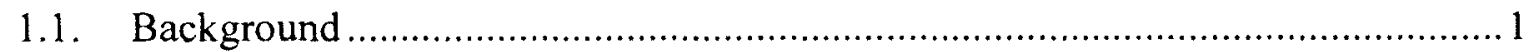

1.2. Problem Definition............................................................................ 2

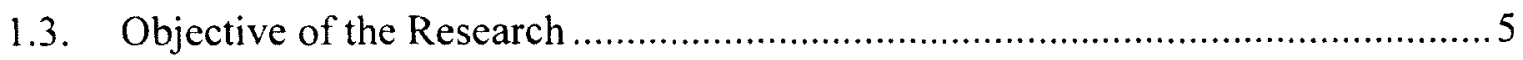

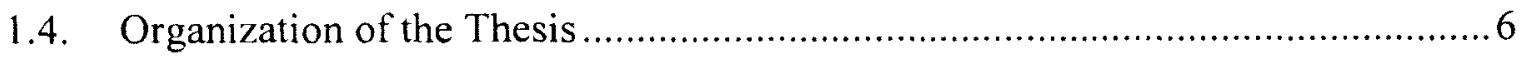

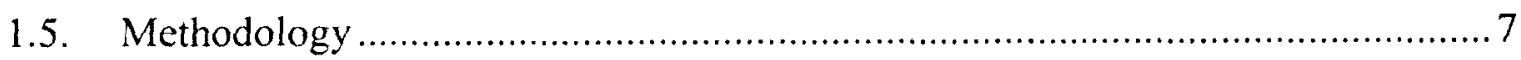

Chapter 2. Electrochemical Impedance Spectroscopy (EIS) .................................9

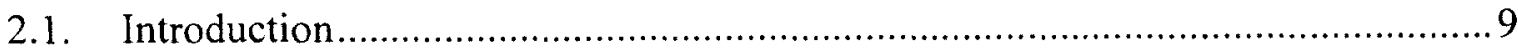

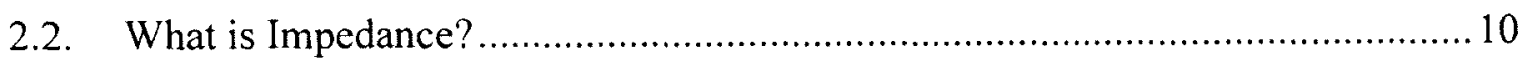

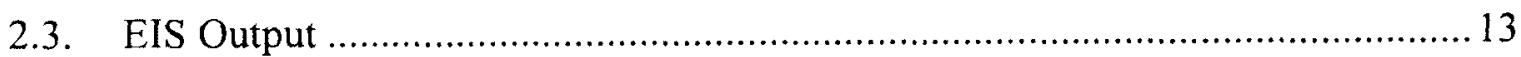

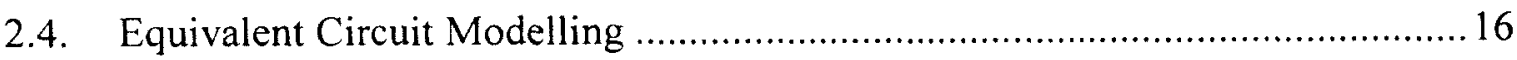

2.5. The Use of EIS to Study Barrier Coatings ............................................. 22

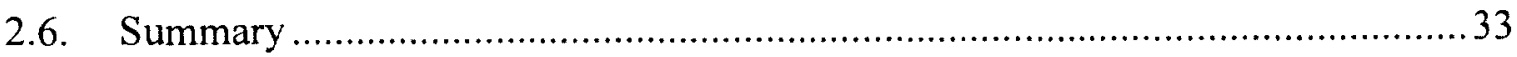

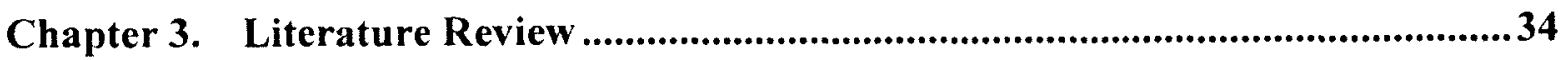

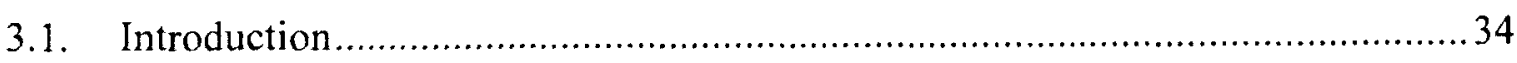

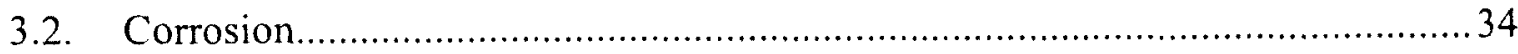

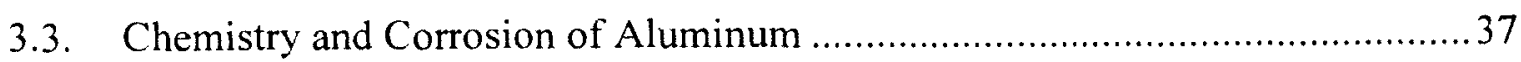

3.3.1. Chemistry of Aluminum (Aluminum Oxide) ................................... 37

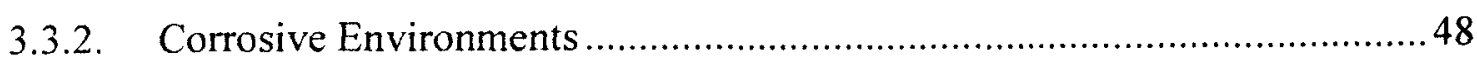




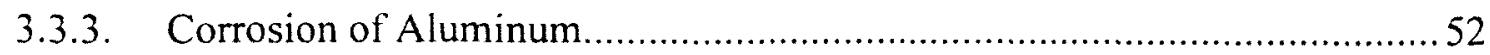

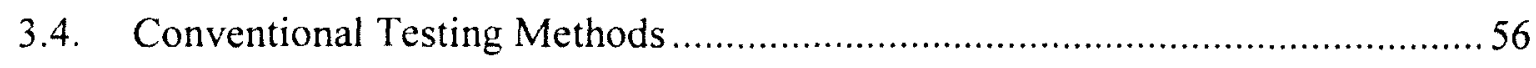

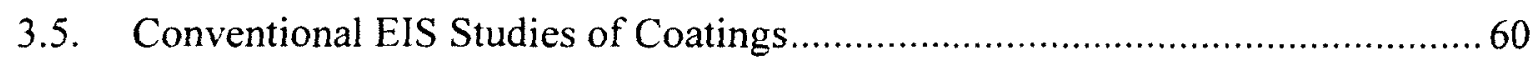

3.6. Previous Studies on Aluminum Food/Beverage Containers...............................65

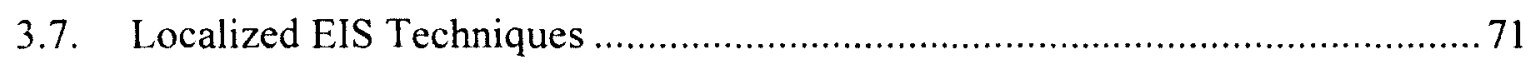

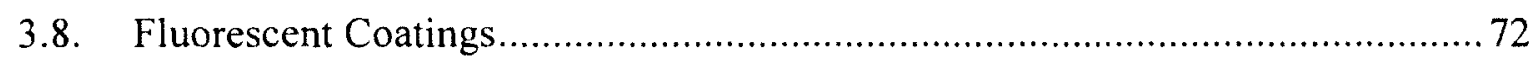

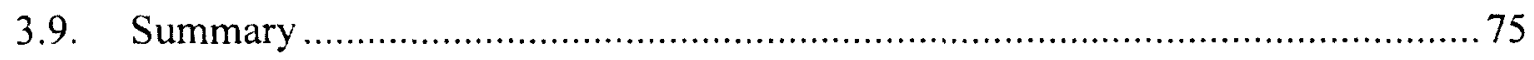

Chapter 4. Experimental Setup and Equipment Used .................................................77

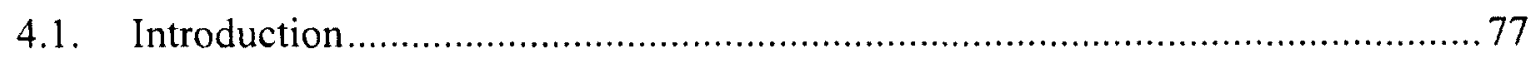

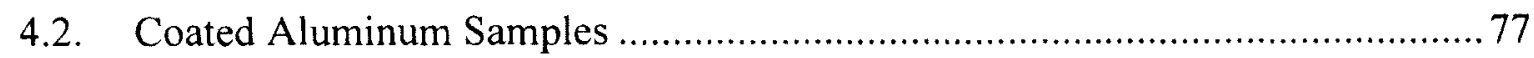

4.3. Experimental Procedure .............................................................................. 77

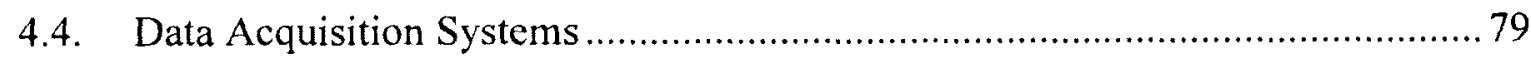

4.5. Noise Elimination in Solartron Instruments .................................................. 79

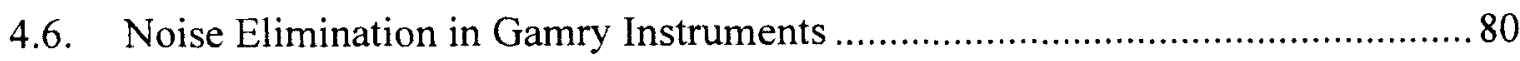

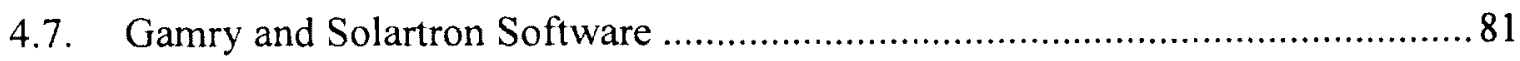

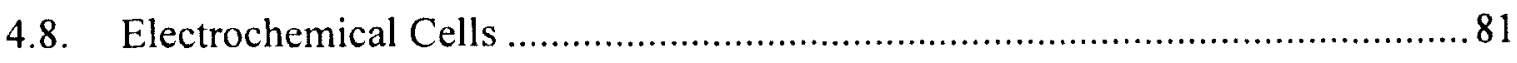

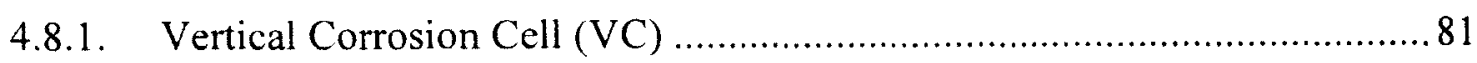

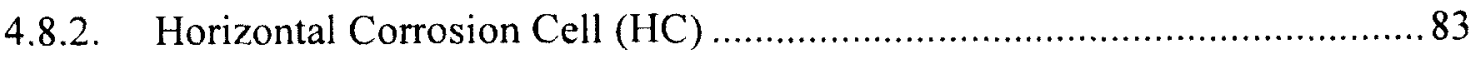

Chapter 5. Preliminary Experiments..........................................................................84

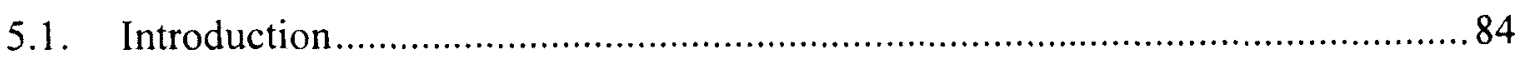

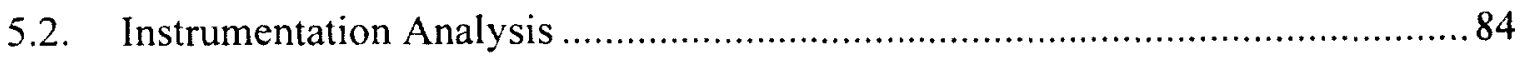

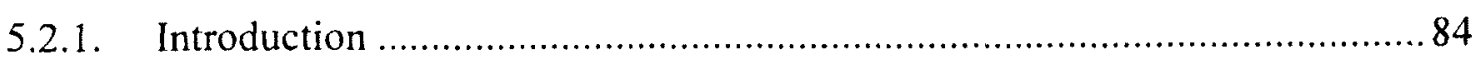

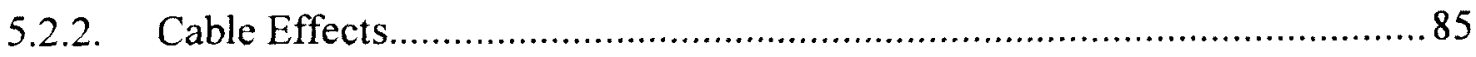

5.2.3. Electrical Noise Interference and Instrument Limits.................................90 


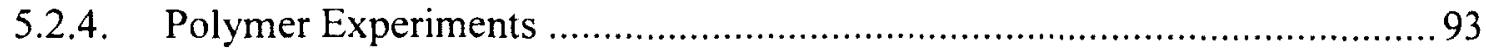

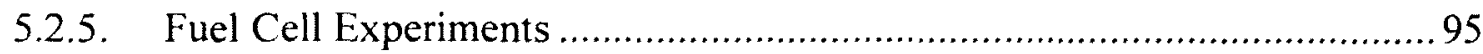

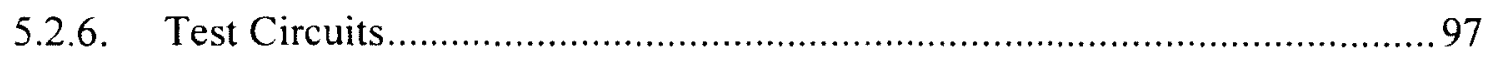

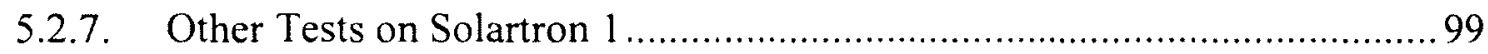

5.2.8. Coated Aluminum Samples .............................................................. 101

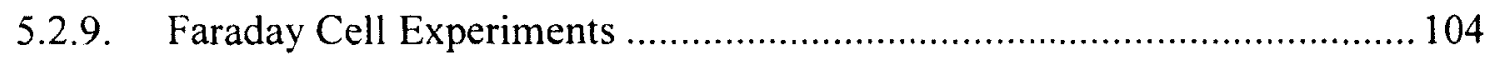

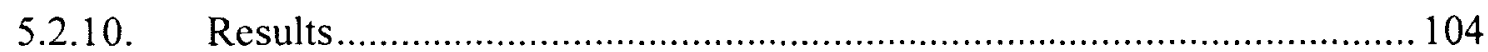

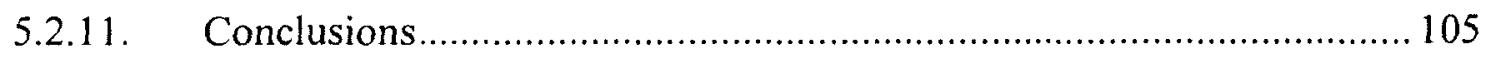

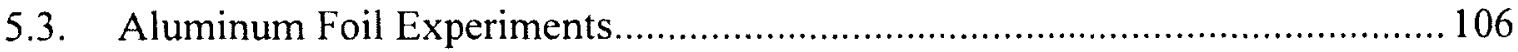

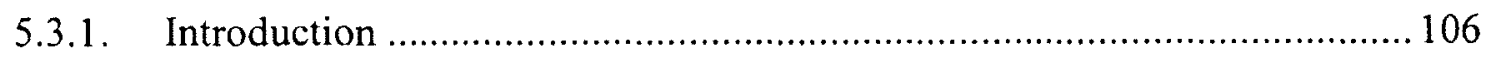

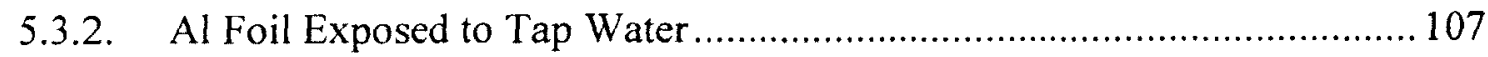

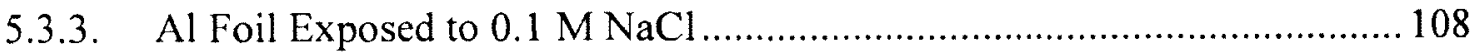

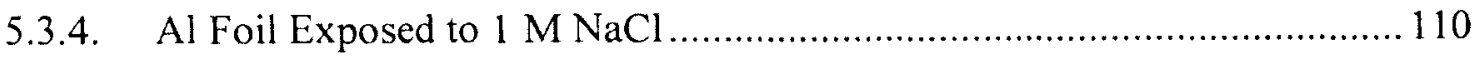

5.3.5. DC Polarization Experiments …………............................................. 114

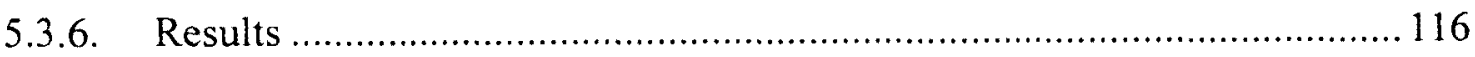

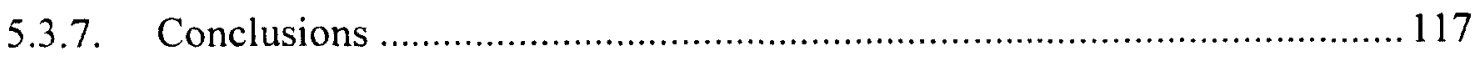

5.4. The Effect of Varying the Sample Size and Electrolyte Concentration ...........118

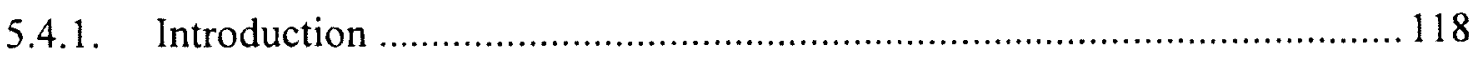

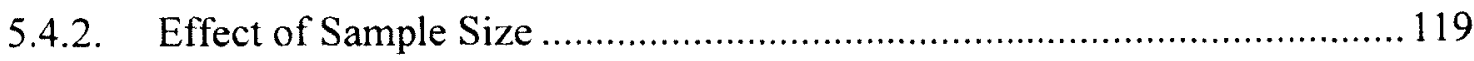

5.4.3. Differences in Bode Plot for Good and Poor Coatings ............................122

5.4.4. Effect of Changing Electrolyte Concentration ........................................ 125

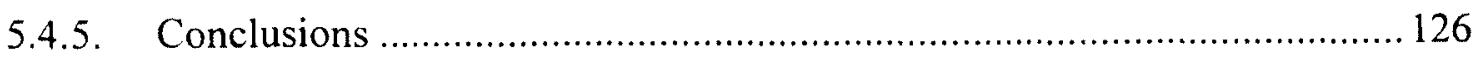

Chapter 6. Results for EIS of Coated Aluminum Samples ........................................ 128

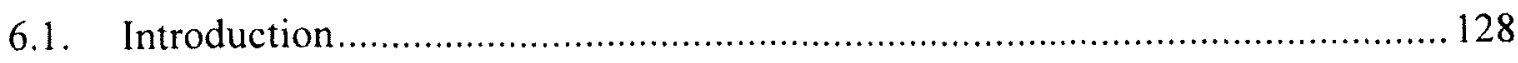




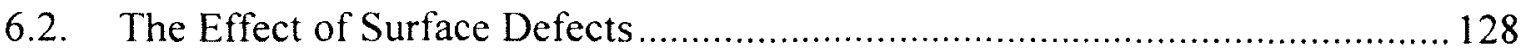

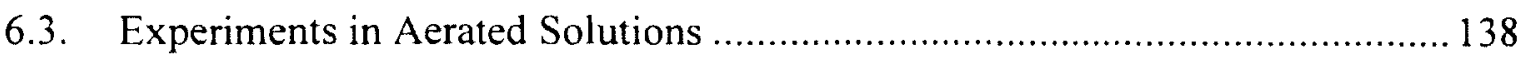

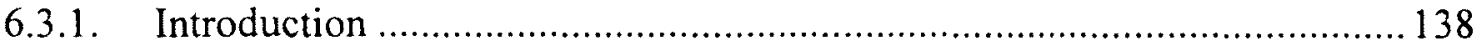

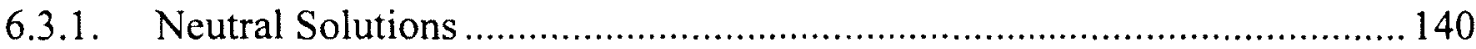

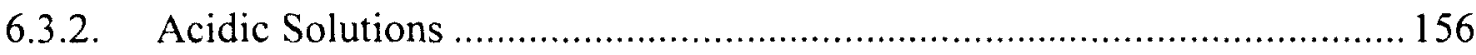

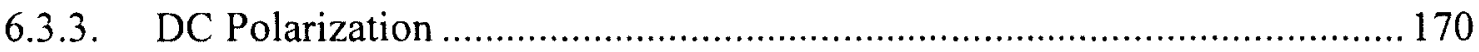

6.4. Experiments in Deaerated Solutions ......................................................... 172

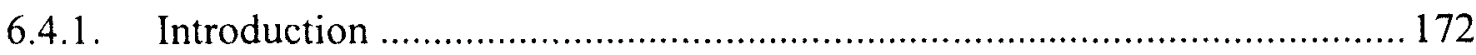

6.4.2. $1 \mathrm{M} \mathrm{NaCl}(\mathrm{pH} 8)$ Deaerated with Dry Ice ............................................ 173

6.4.3. $1 \mathrm{M} \mathrm{NaCl}(\mathrm{pH}$ 8) Deaerated with Nitrogen Gas ……….......................... 175

6.4.4. $1 \mathrm{M} \mathrm{HCl}(\mathrm{pH} 0.1)$ Deaerated with Dry Ice .......................................... 179

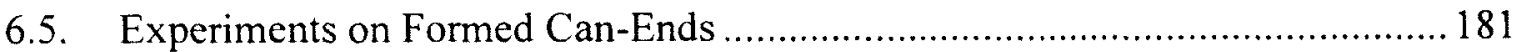

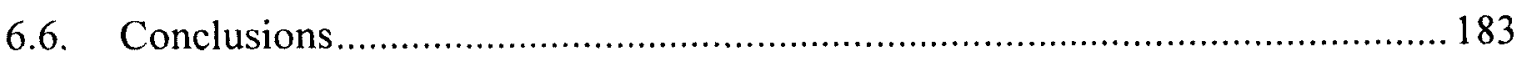

6.6.1. Conclusions from the Deliberate Flaws Experiments .............................. 183

6.6.2. Conclusions from Experiments in Aerated Solutions............................... 185

6.6.3. Conclusions from Experiments in Deaerated Solutions ............................ 186

6.6.4. Conclusions from Can-End Experiments ............................................. 186

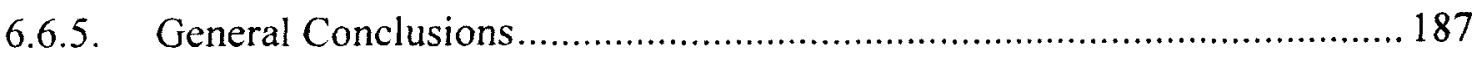

Chapter 7. Conclusions and Recommendations.......................................................... 189

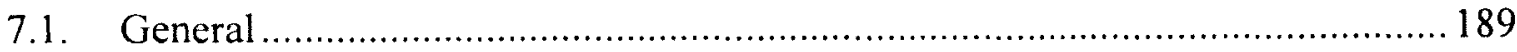

7.2. Conclusions from Preliminary Experiments .................................................. 191

7.3. Conclusions from Experiments in Aerated Solutions ....................................... 193

7.4. Conclusions from Can-End Experiments........................................................ 196

7.5. Conclusions from Experiments in Deaerated Solutions …….......................... 196 


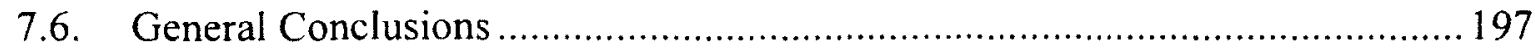

7.7. Recommended Testing Procedure ................................................. 200

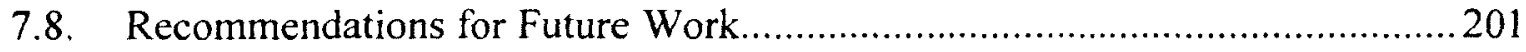

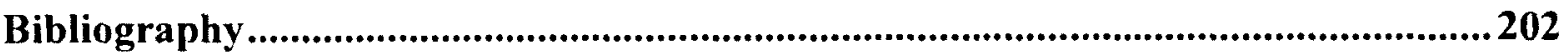




\section{List of Tables}

Table 5-1 Component values and their percentages of error, as fitted to the circuit data

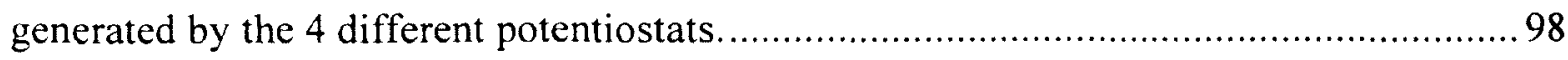
Table 5-2. Calculated capacitance values, using fitting software, for the coated aluminum

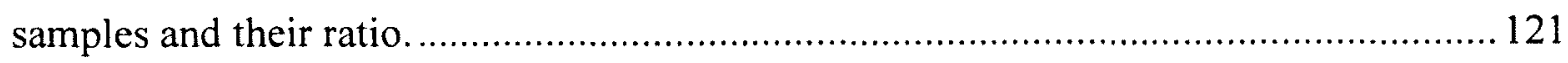

Table 6-1 Observations from placing can ends in $1 \mathrm{M} \mathrm{HCl}$ solution............................ 181 


\section{List of Illustrations}

Figure 2-1 Schematic of an electrochemical cell used to carry out EIS measurements on coatings. 10

Figure 2-2 A small sinusoidal potential excitation and its current response in an $\mathrm{AC}$ system [5]. 11

Figure 2-3 Typical Bode plot of an EIS scan. 14

Figure 2-4 Vector representation of EIS data. 15

Figure 2-5 Nyquist representation of EIS data shown in Figure 2-3. 16

Figure 2-6 A resistor and a capacitor in series

Figure 2-7 A resistor and a capacitor in parallel

Figure 2-8 EIS data for a coated aluminum sample exposed to $1 \mathrm{M} \mathrm{NaCl}(\mathrm{pH} 8)$ for 160 hours. As penetration of the coating occurs, the impedance at low frequencies continuously drops, changing from a capacitor to a resistor at ever higher frequencies. The phase angle, originally $-90^{\circ}$ across the spectrum, increases from $-90^{\circ}$ to $0^{\circ}$ at ever higher frequencies.

Figure 2-9 Schematic of the Randles circuit 24

Figure 2-10 Simulated Bode plot for an intact coating represented with a Randles circuit with $\mathrm{R}_{\mathrm{sol}}=10 \Omega, \mathrm{C}_{\mathrm{c}}=1 \mathrm{nF}$ and $\mathrm{R}_{\mathrm{c}}=1 \mathrm{~T} \Omega$. 26

Figure 2-11 Simulated Nyquist plot for an intact coating represented with a Randles circuit with $\mathrm{R}_{\text {sol }}=10 \Omega, \mathrm{C}_{\mathrm{c}}=1 \mathrm{nF}$ and $\mathrm{R}_{\mathrm{c}}=1 \mathrm{~T} \Omega$. 27

Figure 2-12 Simulated Bode plot for a coating with solution penetrating the pores represented with a Randles circuit with $\mathrm{R}_{\mathrm{sol}}=10 \Omega, \mathrm{C}_{\mathrm{c}}=10 \mathrm{nF}$ and $\mathrm{R}_{\mathrm{c}}=100 \mathrm{M} \Omega$. 28 
Figure 2-13 Simulated Nyquist plot for a coating with solution penetrating the pores represented with a Randles circuit with $R_{\text {sol }}=10 \Omega, C_{c}=10 \mathrm{nF}$ and $R_{c}=100 \mathrm{M} \Omega$.

Figure 2-14 Electrical circuit model for a failed coating.

Figure 2-15 Simulated Bode plot for a coating with a freely corroding substrate represented by the circuit shown in Figure 2-14 with $R_{\text {sol }}=10 \Omega, \quad C_{c}=10 \mathrm{nF}, R_{c}=100$ $\mathrm{k} \Omega, \mathrm{C}_{\mathrm{dl}}=100 \mathrm{nF}$ and $\mathrm{R}_{\mathrm{ct}}=1 \mathrm{M} \Omega$. 30

Figure 2-16 Simulated Nyquist plot for a coating with a freely corroding substrate represented by the circuit shown in Figure $2-14$ with $R_{s o l}=10 \Omega, \quad C_{c}=10 \mathrm{nF}, R_{c}=100$ $\mathrm{k} \Omega, \mathrm{Cdl}=100 \mathrm{nF}$ and $\mathrm{R}_{\mathrm{ct}}=1 \mathrm{M} \Omega$. 30

Figure 2-17 Simulated Bode plot for a severely damaged coating represented by the circuit shown in Figure 2-14 with $R_{\text {sol }}=10 \Omega, C_{c}=10 n F, R_{c}=1 \mathrm{k} \Omega, \quad C_{d l}=1 \mu F$ and $R_{c t}=10$ $\mathrm{k} \Omega$. 32

Figure 2-18 Simulated Nyquist plot for a severely damaged coating represented by the circuit shown in Figure 2-14 with $R_{\text {sol }}=10 \Omega, C_{c}=10 \mathrm{nF}, R_{c}=1 \mathrm{k} \Omega, \quad C_{\mathrm{dl}}=1 \mu \mathrm{F}$ and $R_{\mathrm{ct}}=10$ $\mathrm{k} \Omega$. 32

Figure 3-1 Schematic representation of pitting corrosion of an aluminum sample [17] ...36 Figure 3-2 Pourbaix diagram for aluminum in water at $25^{\circ} \mathrm{C}$ [15]. 38

Figure 3-3 Schematic representation of typical polarization curves: (a) cathodic and anodic half-cell reactions demonstrating half-cell potentials $e_{\mathrm{H}+/ \mathrm{H} 2}$ and $\mathrm{e}_{\mathrm{M} / \mathrm{M}+}$, and halfcell exchange current densities $\mathrm{i}_{\mathrm{O}, \mathrm{H}+/ \mathrm{H} 2}$ and $\mathrm{i}_{\mathrm{O}, \mathrm{M} / \mathrm{M}+}$. These give a corrosion potential $\mathrm{E}_{\mathrm{corr}}$ and corrosion current $i_{\text {corr }}$ with an overpotential of $\varepsilon_{c}$ compared to the mixedpotential/equilibrium (corrosion) potential; (b) simulated experimental polarization curves derived from (a) [19]. 
Figure 3-4 Evans diagram for active-passive metals like aluminum [21]. .46

Figure 3-5 Effect of oxidizer concentration on the electrochemical behavior of an activepassive metal [19]. 50

Figure 3-6 Schematic representation showing the relationship between $\mathrm{pH}$ of several solutions and the corresponding corrosion rate of aluminum [15]. .52

Figure 3-7 Sample polarization curve demonstrating the formation of pits [20]. .54

Figure 3-8 Circuits used to model EIS data of coatings on metals: (a) intact coating; (b) damaged coating where the electrolyte can reach the metal surface; (c) using constantphase elements (CPE) instead of pure capacitors to model non-uniform distribution of the coatings and surface roughness; (d) addition of a Warburg element to model the impedance associated with diffusion and mass transfer processes [9].

Figure 3-9 Simulated Bode plot using circuits represented in Figure 3-8 [9]. Note the increase in complexity in the plot with sample damage

Figure 3-10 Simulated Nyquist plot using circuits represented in Figure 3-8 [9]. Note the appearance of a second semicircle with the sample damage. .64

Figure 3-11 Schematic representation of a Bode plot showing the break-point frequencies. 66

Figure 3-12 Bode plot representing the \% ideal method used to represent EIS data [30].67 Figure 3-13 Impedance spectra for coated aluminum before and after introducing a defect [31] .68

Figure 3-14 Bode phase-angle plots for uncoated and coated (with defect at 193h) aluminum [31]. Note the appearance of positive phase angles at the low end of the frequency spectrum which is discussed in section 5.3 and chapter 6 .

69 
Figure 3-15 Circuit model proposed to represent coated aluminum in chloride containing aqueous media [31].

Figure 3-16 Images of an aluminum sample coated with a FD1-containing clear epoxy coating after (a) 2 days and (b) 3 days of exposure to $3.5 \% \mathrm{NaCl}$ solution. Top row: digital camera images taken through the microscope eyepiece under UV light. Bottom row: images of the same area taken through the microscope eyepiece under natural light [18] 74

Figure 4-1 Vertical corrosion cell. The sample is shown under the black O-ring at the bottom of the glass cylinder. The sample diameter is $6.35 \mathrm{~cm}$. 82

Figure 4-2 Horizontal corrosion cell. The volume of the cell is $250 \mathrm{ml}$ and the working electrode area is $1 \mathrm{~cm}^{2}$. 83

Figure 5-1 Impedance $(\Omega)$ measurements $\left(\mathrm{HC}, 1.0 \pm 0.1 \mathrm{~cm}^{2}\right.$ ) for coated aluminum using Solartron 1 with different cables. 86

Figure 5-2 Phase angle measurements (HC, $1.0 \pm 0.1 \mathrm{~cm}^{2}$ ) for coated aluminum using Solartron 1 with different cables. 87

Figure 5-3 Impedance $(\Omega)$ measurements $\left(\mathrm{HC}, 1.0 \pm 0.1 \mathrm{~cm}^{2}\right.$ ) for polymer using Gamry 1 with good and bad cables. 88

Figure 5-4 Impedance $(\Omega)$ measurements $\left(\mathrm{HC}, 1.0 \pm 0.1 \mathrm{~cm}^{2}\right)$ for polymer using Gamry 2 with good and bad cables. .88 Figure 5-5 Phase angle measurements $\left(\mathrm{HC}, 1.0 \pm 0.1 \mathrm{~cm}^{2}\right)$ for polymer using Gamry 1 with good and bad cables. 89

Figure 5-6 Phase angle measurements $\left(\mathrm{HC}, 1.0 \pm 0.1 \mathrm{~cm}^{2}\right)$ for polymer using Gamry 2 with good and bad cables. 
Figure 5-7 Open-lead spectra for Solartron 1 and Solartron 2................................. 91

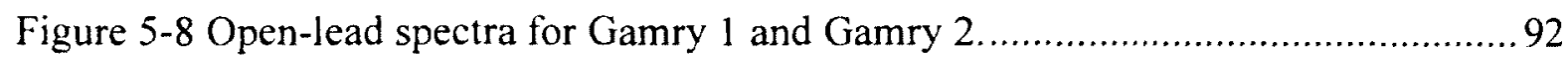

Figure 5-9 Open-lead spectra for Gamry 1 at location 1 and location 2...................92

Figure 5-10 Impedance $(\Omega)$ measurements $\left(\mathrm{HC}, 1.0 \pm 0.1 \mathrm{~cm}^{2}\right)$ for polymer in water using

Solartron 1, Solartron 2, Gamry 1 and Gamry 2............................................ 93

Figure 5-11 Phase angle measurements $\left(\mathrm{HC}, 1.0 \pm 0.1 \mathrm{~cm}^{2}\right)$ for polymer in water using

Solartron 1, Solartron 2, Gamry 1 and Gamry 2. ......................................... 94

Figure 5-12 Impedance $(\Omega)$ measurements for fuel cell...................................... 96

Figure 5-13 Phase angle measurements for fuel cell ....................................... 96

Figure 5-14 Components of test circuit. ........................................................... 97

Figure 5-15 Impedance $(\Omega)$ measurements for test circuit.................................. 99

Figure 5-16 Phase angle measurements for test circuit. ....................................... 99

Figure 5-17 Phase Angle measurements for circuit elements using Solartron 1.......... 100

Figure 5-18 Impedance $(\Omega)$ measurements for circuit elements using Solartron $1 \ldots \ldots . .100$

Figure 5-19 Impedance $(\Omega)$ measurements $\left(\mathrm{HC}, 1.0 \pm 0.1 \mathrm{~cm}^{2}\right)$ for intact coated aluminum sample.

Figure 5-20 Phase angle measurements $\left(\mathrm{HC}, 1.0 \pm 0.1 \mathrm{~cm}^{2}\right)$ for intact coated aluminum sample.

Figure 5-21 A Bode plot (using Solartron 1, VC, $34.0 \pm 0.5 \mathrm{~cm}^{2}$ ) showing the impedance and phase angle for aluminum kitchen foil exposed to tap water. Compare this with data for an uncoated sample in Figure 3-14. 108

Figure 5-22 A Bode plot (using Solartron 1, VC, $34.0 \pm 0.5 \mathrm{~cm}^{2}$ ) showing the impedance and phase angle for aluminum kitchen foil exposed to $0.1 \mathrm{M} \mathrm{NaCl}$. 110 
Figure 5-23 Impedance $(\Omega)$ measurements (using Solartron 1, VC, $34.0 \pm 0.5 \mathrm{~cm}^{2}$ ) for aluminum kitchen foil exposed to $1 \mathrm{M} \mathrm{NaCl}(\mathrm{pH}$ 8). Note the increase in the high frequency impedance and drop in the low frequency impedance. 111

Figure 5-24 Phase angle measurements (using Solartron 1, VC, $34.0 \pm 0.5 \mathrm{~cm}^{2}$ ) for aluminum kitchen foil exposed to $1 \mathrm{M} \mathrm{NaCl}(\mathrm{pH} \mathrm{8}$ ). (the positive phase angle at the highest frequencies is attributed to inductance in the cables as previously discussed in section 5.2.2) 112

Figure 5-25 Nyquist plot (using Solartron 1, VC, $34.0 \pm 0.5 \mathrm{~cm}^{2}$ ) for aluminum kitchen foil exposed to $1 \mathrm{M} \mathrm{NaCl}(\mathrm{pH}$ 8). Note the inductive effects (positive imaginary impedance) appearing with sample degradation.

Figure 5-26 Photograph showing pinholes (numerous white dots) in aluminum foil after exposure to $1 \mathrm{M} \mathrm{NaCl}$. 114

Figure 5-27 Bode plot (using Solartron 1, VC, $34.0 \pm 0.5 \mathrm{~cm}^{2}$ ) showing the effects of the application of small $\mathrm{DC}$ voltages to aluminum kitchen foil in $1 \mathrm{M} \mathrm{NaCl}$ 115 Figure 5-28 Impedance $(\Omega)$ measurements for coated-aluminum samples to show the effect of varying the sample surface area. 120 Figure 5-29 Potentiodynamic scan using Solartron 2 for the high-impedance epoxy polymer 124

Figure 5-30 Potentiodynamic scan using Solartron 2 for an intact coated-aluminum sample. 125

Figure 5-31 Bode plot using Gamry $1\left(\mathrm{HC}, 1.0 \pm 0.1 \mathrm{~cm}^{2}\right)$ for intact coated aluminum sample in two solutions, $1 \mathrm{M} \mathrm{NaCl}$ and $1 \mathrm{M} \mathrm{HCl}$. 126 
Figure 6-1 Bode plot (using Solartron 2, $\mathrm{HC}, 1.0 \pm 0.1 \mathrm{~cm}^{2}$ ) for a coated aluminum sample, immersed in $0.1 \mathrm{M} \mathrm{NaCl}(\mathrm{pH} 8)$, before introducing a surface defect. Note the very high impedance at low frequencies, and the constant phase angle of $-90^{\circ}$, indicating that this is a good capacitor, over the complete frequency range. 129

Figure 6-2 Potentiodynamic scan using Solartron $2\left(\mathrm{HC}, 1.0 \pm 0.1 \mathrm{~cm}^{2}\right)$ for a coated aluminum sample in Figure 6-1 (before introducing a surface defect). This is a low current density, at the detection level of the potentiostat. 130

Figure 6-3 Impedance $(\Omega)$ plot using Solartron $2\left(\mathrm{HC}, 1.0 \pm 0.1 \mathrm{~cm}^{2}\right)$ for a coated aluminum sample before a surface scratch in $0.1 \mathrm{M} \mathrm{NaCl}(\mathrm{pH} 8)$, and after introducing a surface scratch (in $0.1 \mathrm{M} \mathrm{NaCl}(\mathrm{pH} 8)$ and $1 \mathrm{M} \mathrm{NaCl}(\mathrm{pH} \mathrm{8})$ ). Note the drop in impedance by several orders of magnitude after the scratch, and by changing solution concentration.132 Figure 6-4 Phase angle plot using Solartron $2\left(\mathrm{HC}, 1.0 \pm 0.1 \mathrm{~cm}^{2}\right)$ for a coated aluminum sample before and after a surface scratch, and after changing the electrolyte from $0.1 \mathrm{M}$ $\mathrm{NaCl}(\mathrm{pH} 8)$ to $1 \mathrm{M} \mathrm{NaCl}(\mathrm{pH}$ 8). Note that the magnitude of the positive phase angle increased with increasing solution conductivity. 132

Figure 6-5 Potentiodynamic scan using Solartron $2\left(\mathrm{HC}, 1.0 \pm 0.1 \mathrm{~cm}^{2}\right)$ for a coated aluminum sample, in $0.1 \mathrm{M} \mathrm{NaCl}(\mathrm{pH} 8)$ before scratch and in $1 \mathrm{M} \mathrm{NaCl}(\mathrm{pH} \mathrm{8)}$ after a surface scratch. 133

Figure 6-6 Nyquist plot using Solartron $2\left(\mathrm{HC}, 1.0 \pm 0.1 \mathrm{~cm}^{2}\right)$ for a coated aluminum sample before and after a surface scratch in $0.1 \mathrm{M} \mathrm{NaCl}(\mathrm{pH}$ 8) and in $1 \mathrm{M} \mathrm{NaCl}(\mathrm{pH}$ 8) after a surface scratch. The unscratched sample behaves like a capacitor [12]. See also Figure 6-7 for a better view of the plots for the scratched sample. 137 
Figure 6-7 Nyquist plot using Solartron $2\left(\mathrm{HC}, 1.0 \pm 0.1 \mathrm{~cm}^{2}\right)$ for a coated aluminum sample after a surface scratch in $0.1 \mathrm{M} \mathrm{NaCl}(\mathrm{pH} \mathrm{8)}$ and in $1 \mathrm{M} \mathrm{NaCl}(\mathrm{pH} \mathrm{8})$. Note the pseudo-inductive effects (positive Zimag) observed in the plot. Note the decrease in magnitude of both Zreal and Zimg when the electrolyte was more concentrated.......... 138

Figure 6-8 SEM image of an intact coated-aluminum sample.

Figure 6-9 Impedance $(\Omega)$ measurements using Gamry $1\left(\mathrm{HC}, 1.0 \pm 0.1 \mathrm{~cm}^{2}\right)$ for coated aluminum sample in $1 \mathrm{M} \mathrm{NaCl}(\mathrm{pH} 8)$. Note the passivation occurring that leads to an increase in the impedance.

Figure 6-10 Phase angle measurements using Gamry $1\left(\mathrm{HC}, 1.0 \pm 0.1 \mathrm{~cm}^{2}\right)$ for coated aluminum sample in $1 \mathrm{M} \mathrm{NaCl}(\mathrm{pH} 8)$. Note the disappearance of spikes and the purely capacitive plot after 1 hour of exposure. 142

Figure 6-11 Impedance $(\Omega)$ measurements using Gamry $1\left(\mathrm{HC}, 1.0 \pm 0.1 \mathrm{~cm}^{2}\right)$ for coated aluminum sample in $1 \mathrm{M} \mathrm{NaCl}(\mathrm{pH}$ 8). Note the rising and falling impedance indicating some intermittent passivation occurring on the surface of the metal substrate and intermittent blocking of the conductive paths.

Figure 6-12 Phase angle measurements using Gamry 1 ( $\mathrm{HC}, 1.0 \pm 0.1 \mathrm{~cm}^{2}$ ) for coated aluminum sample in $1 \mathrm{M} \mathrm{NaCl}$. Note the intermittent passivation occurring on the surface of the metal indicated by changes in the phase angles from capacitive to resistive, and back to capacitive. 144

Figure 6-13 Impedance $(\Omega)$ measurements using Gamry $1\left(\mathrm{HC}, 1.0 \pm 0.1 \mathrm{~cm}^{2}\right)$ for coated aluminum sample in $1 \mathrm{M} \mathrm{NaCl}(\mathrm{pH} \mathrm{8})$. Note the rise and fall in impedance with exposure and the increasing capacitive-to-resistive transition frequency. 145 
Figure 6-14 Phase angle measurements using Gamry $1\left(\mathrm{HC}, 1.0 \pm 0.1 \mathrm{~cm}^{2}\right)$ for coated aluminum sample in $1 \mathrm{M} \mathrm{NaCl}(\mathrm{pH} 8)$. Note the increasing capacitive-to-resistive transition frequency with exposure time. 146

Figure 6-15 Oxide cloud observed in the horizontal cell (Figure 4-2) during the immersion of a coated aluminum sample in $1 \mathrm{M} \mathrm{NaCl}(\mathrm{pH} 8)$.

Figure 6-16 Bubbles on the sample are observed in the horizontal cell (Figure 4-2) during the exposure of a coated aluminum sample in $1 \mathrm{M} \mathrm{NaCl}(\mathrm{pH} 8)$. 148

Figure 6-17 Condition of a coated aluminum sample after exposure to $1 \mathrm{M} \mathrm{NaCl}(\mathrm{pH} 8)$ for $161 \mathrm{hrs}$ in the horizontal cell (Figure 4-2).

Figure 6-18 Impedance $(\Omega)$ measurements using Solartron $1\left(\mathrm{VC}, 34.0 \pm 0.5 \mathrm{~cm}^{2}\right.$ ) for a coated aluminum sample in $0.1 \mathrm{M} \mathrm{NaCl}(\mathrm{pH} 8)$. Note the sudden increase in impedance at $7 \mathrm{hrs}$, indicating passivation. 150

Figure 6-19 Phase angle measurements using Solartron $1\left(\mathrm{VC}, 34.0 \pm 0.5 \mathrm{~cm}^{2}\right)$ for a coated aluminum sample in $0.1 \mathrm{M} \mathrm{NaCl}(\mathrm{pH} 8)$.

Figure 6-20 Impedance $(\Omega)$ measurements using Solartron $1\left(\mathrm{VC}, 34.0 \pm 0.5 \mathrm{~cm}^{2}\right)$ for a coated aluminum sample in $0.1 \mathrm{M} \mathrm{NaCl}(\mathrm{pH} 8)$. Note the sudden drop in impedance at 26 hrs suggesting the opening of a conductive path in the coating. 151 Figure 6-21 Phase angle measurements using Solartron $1\left(\mathrm{VC}, 34.0 \pm 0.5 \mathrm{~cm}^{2}\right)$ for a coated aluminum sample in $0.1 \mathrm{M} \mathrm{NaCl}(\mathrm{pH} 8)$.

Figure 6-22 Oxide trees observed to emerge from pitting sites, in the vertical cell (Figure 4-1), during the immersion of a coated aluminum sample in $0.1 \mathrm{M} \mathrm{NaCl}(\mathrm{pH} \mathrm{8})$. Some of the oxide was seen flaoting in the solution. 153 
Figure 6-23 Condition of a coated aluminum sample after exposure to $0.1 \mathrm{M} \mathrm{NaCl}(\mathrm{pH} 8)$ for 330 hours in the vertical cell (Figure 4-1). Pits and through holes were found on the surface of the sample. 154

Figure 6-24 SEM image of a pit in the sample exposed to $0.1 \mathrm{M} \mathrm{NaCl}(\mathrm{pH} 8) \ldots \ldots \ldots \ldots . .155$

Figure 6-25 SEM image of a pit in the sample exposed to $0.1 \mathrm{M} \mathrm{NaCl}(\mathrm{pH} 8)$............. 156 Figure 6-26 Bode plot (using Gamry 1) for sample in $1 \mathrm{M} \mathrm{HCl}(\mathrm{pH} \mathrm{0.1)}$ in $\mathrm{HC}(1.0 \pm 0.1$ $\mathrm{cm}^{2}$ ). No changes were observed in the Bode plot for 850 hours. 157

Figure 6-27 Nyquist plot (using Gamry 1) for sample in $1 \mathrm{M} \mathrm{HCl} \mathrm{(pH} \mathrm{0.1)} \mathrm{in} \mathrm{HC}$ $\left(1.0 \pm 0.1 \mathrm{~cm}^{2}\right)$ 158

Figure 6-28 Impedance $(\Omega)$ measurements using Gamry $1\left(\mathrm{VC}, 34.0 \pm 0.5 \mathrm{~cm}^{2}\right.$ ) for a coated aluminum sample in $1 \mathrm{M} \mathrm{HCl}(\mathrm{pH} 0.1)$. 159

Figure 6-29 Phase angle measurements using Gamry $1\left(\mathrm{VC}, 34.0 \pm 0.5 \mathrm{~cm}^{2}\right.$ ) for coated aluminum sample in $1 \mathrm{M} \mathrm{HCl}(\mathrm{pH} 0.1)$ 160

Figure 6-30 Bubbles starting to form on the surface of a sample exposed to $1 \mathrm{M} \mathrm{HCl}(\mathrm{pH}$ 0.1 ) in the vertical cell (Figure 4-1) 161

Figure 6-31 Bubbles on the surface of a sample exposed to $1 \mathrm{M} \mathrm{HCl}(\mathrm{pH} 0.1)$ in the vertical cell (Figure 4-1). 161

Figure 6-32 Condition of a coated aluminum sample after exposure to $1 \mathrm{M} \mathrm{HCl}(\mathrm{pH} 0.1)$ for 1200 hours in the vertical cell (Figure 4-1). Many pits and through holes formed on the surface. 162

Figure 6-33 SEM image of a pit in the sample exposed to $1 \mathrm{M} \mathrm{HCl}(\mathrm{pH} 0.1)$. Note the state of the coating around the pitting site: Some of the coating appears to be missing. 163 
Figure 6-34 Impedance $(\Omega)$ measurements using Solartron $2\left(\mathrm{HC}, 1.0 \pm 0.1 \mathrm{~cm}^{2}\right)$ for coated aluminum sample in $\mathrm{HCl}(\mathrm{pH} 2.6)$. Note the drop in impedance at 53 hours of exposure, probably indicating a pre-existing flaw. 165

Figure 6-35 Phase angle measurements using Solartron $2\left(\mathrm{HC}, 1.0 \pm 0.1 \mathrm{~cm}^{2}\right)$ for coated aluminum sample in $\mathrm{HCl}(\mathrm{pH} 2.6)$. 165

Figure 6-36 Impedance $(\Omega)$ measurements using Solartron $2\left(\mathrm{HC}, 1.0 \pm 0.1 \mathrm{~cm}^{2}\right)$ for coated aluminum sample in $\mathrm{HCl}(\mathrm{pH} 2.6)$. Note the drop in impedance (breach in the oxide) at $138 \mathrm{hrs}$ and the increase in impedance (passivation) at $155 \mathrm{hrs}$. 166

Figure 6-37 Phase angle measurements using Solartron $2\left(\mathrm{HC}, 1.0 \pm 0.1 \mathrm{~cm}^{2}\right)$ for coated aluminum sample in $\mathrm{HCl}(\mathrm{pH} 2.6)$. 166

Figure 6-38 Impedance $(\Omega)$ measurements using Solartron $2\left(\mathrm{HC}, 1.0 \pm 0.1 \mathrm{~cm}^{2}\right)$ for coated aluminum sample in $\mathrm{HCl}(\mathrm{pH} 2.6)$. Note the increase in high-frequency impedance at $288 \mathrm{hrs}$ and the increasing complexity of the plot. 168

Figure 6-39 Phase angle measurements using Solartron $2\left(\mathrm{HC}, 1.0 \pm 0.1 \mathrm{~cm}^{2}\right)$ for coated aluminum sample in $\mathrm{HCl}(\mathrm{pH} 2.6)$. Note the increasing complexity in the phase plot... 169 Figure 6-40 Condition of a coated aluminum sample after exposure to $\mathrm{pH} 2.6 \mathrm{HCl}$ for 650 hours in the horizontal cell (Figure 4-2). Note the oxide formed in the pits. There was no penetration.

170

Figure 6-41 Impedance $(\Omega)$ measurements using Gamry 1 (HC, $1.0 \pm 0.1 \mathrm{~cm}^{2}$ ) demonstrating the effects of applying small DC voltages to coated aluminum in $\mathrm{pH} 0.2$ $\mathrm{HCl}$. Note the increase in impedance with the application of a cathodic (negative) voltage. 
Figure 6-42 Phase angle measurements using Gamry $1\left(\mathrm{HC}, 1.0 \pm 0.1 \mathrm{~cm}^{2}\right)$ demonstrating the effects of applying small DC voltages to coated aluminum in $\mathrm{pH} 0.2 \mathrm{HCl}$. With the application of a cathodic (negative) voltage, spikes disappeared and the phase plots are more capacitive 172

Figure 6-43 Impedance $(\Omega)$ measurements, using Solartron $1\left(\mathrm{HC}, 1.0 \pm 0.1 \mathrm{~cm}^{2}\right)$, for coated aluminum sample in deaerated $1 \mathrm{M} \mathrm{NaCl}(\mathrm{pH} 8)$. Note the downward shift in the impedance plot with exposure. 174

Figure 6-44 Phase angle measurements, using Solartron $1\left(\mathrm{HC}, 1.0 \pm 0.1 \mathrm{~cm}^{2}\right)$, for coated aluminum sample in deaerated $1 \mathrm{M} \mathrm{NaCl}(\mathrm{pH} \sim 8)$. 175

Figure 6-45 Impedance $(\Omega)$ measurements, using Solartron $1\left(\mathrm{HC}, 1.0 \pm 0.1 \mathrm{~cm}^{2}\right)$, for coated aluminum sample in deaerated $1 \mathrm{M} \mathrm{NaCl}(\mathrm{pH} 8)$. Note the downward shift in impedance between 0 and 1650 hours of exposure. After aeration of the solution at 1657 hours, a large downward shift is observed in the impedance plot, 1657 hours, after which it shifted back. 176

Figure 6-46 Phase angle measurements, using Solartron $1\left(\mathrm{HC}, 1.0 \pm 0.1 \mathrm{~cm}^{2}\right)$, for coated aluminum sample in deaerated $1 \mathrm{M} \mathrm{NaCl}(\mathrm{pH} 8)$. After aeration of the solution, a more resistive phase plot is observed, 1657 hours, which went back to being capacitive at 1660 hours. 177

Figure 6-47 Condition of a coated aluminum sample after exposure to $1 \mathrm{M} \mathrm{NaCl}(\mathrm{pH} 8)$ for 2500 hours in the horizontal cell (Figure 4-2). Initially the solution was deaerated with $\mathrm{N}_{2}$ gas, then aerated after 1650 hours. Note that all the aluminum corroded away and only the coating on both sides was left. This is consistent with the build up of an acid solution between the two coating layers. 178 
Figure 6-48 Impedance $(\Omega)$ measurements, using Solartron $1\left(\mathrm{VC}, 34.0 \pm 0.5 \mathrm{~cm}^{2}\right.$ ), for coated aluminum sample in deaerated $1 \mathrm{M} \mathrm{HCl}(\mathrm{pH} 0.1) \ldots$ 180

Figure 6-49 Phase angle measurements, using Solartron 1 (VC, $34.0 \pm 0.5 \mathrm{~cm}^{2}$ ), for coated aluminum sample in deaerated $1 \mathrm{M} \mathrm{HCl}(\mathrm{pH} 0.1)$. 180

Figure 6-50 Sample with coating 1 showed the start of corrosion within 3 days. The corrosion sites, indicated by gas bubbles, are all in areas stressed by stamping of the can end. 182

Figure 6-51 Sample with coating 2 showed discolouration after 3 days and pitting after 17 days. 183 


\section{Chapter 1. Introduction}

\subsection{Background}

When aluminum cans are manufactured, they have a coating applied to the inside and outside to protect the aluminum from corrosive environments.

An aluminum, beverage can is made with two pieces - the can body and the can end (or lid). The manufacturing process starts with coils of aluminum. Can plants use large quantities of aluminum coil every day to make can bodies or ends. Each coil typically weighs about $11,340 \mathrm{~kg}(25,000$ pounds) and, when rolled out flat, can be anywhere from $6,100 \mathrm{~m}$ to $9,100 \mathrm{~m}(20,000$ feet to 30,000 feet) long and $1.5 \mathrm{~m}$ to $1.8 \mathrm{~m}$ (5 to 6 feet) wide [1].

The aluminum coils arrive at the can plant and are loaded one at a time onto an "uncoiler", a machine that unrolls the strip of aluminum at the beginning of the canmaking line and feeds it to the line, where it is first lubricated. Lubrication helps the aluminum flow smoothly during the can shaping processes that follow. A large machine called a cupping press starts the can shaping process. The press cuts circular discs from the aluminum sheet and forms them into shallow cups after which they drop onto the cup conveyor. These two metal-forming operations are performed at high speeds and make 2,500 to 3,750 cups per minute. The scrap (or skeleton) aluminum left over from these operations is removed and recycled [1].

From the cupping press, the cups are drawn into longer cups through a series of iron rings; now the aluminum starts to look like a can. The tops are trimmed off to make them even; each can is the same height and width. A washer cleans and dries the can bodies so they can be decorated, and the cans proceed to a printer, where six to eight 
colors of ink may be placed on a can at the same time. The can spins around as the label is applied. Finally, a coating is applied that makes the outside of the can shiny and protects the newly applied paint and the can's exterior [1].

Next, the can goes to an oven, where the paint and coating are baked onto the can to prevent chipping. The can's interior is then coated with a spray to prevent what is in the can from touching or reacting with the metal, and the can is baked in an oven again to seal the coating onto the can. The top of the can is now made narrow for the can lids to be fitted after the can is filled. A lip is formed, called a flange, which helps seal the lid in place after the soft drink is put in the can. The bottom of the can is also reformed at this point. A machine makes a small dome that helps improve the strength of the container [1].

Finally, all finished cans are tested for leaks. A light tester can find holes smaller than a human hair. The cans are placed on pallets and shipped to soft drink companies. The lids of the cans, called can ends, are made separately and shipped separately to the soft drink companies. As with the can-body manufacturing, the can-end making process starts with a coil of aluminum. The aluminum is uncoiled, lubricated and fed to a machine that makes it into a round shell. The shells are coated with a protective coating and dried. This way, none of the soft drink will actually touch the metal. Next, a machine makes a button on the end where an easy-open tab can be secured into place [1].

\subsection{Problem Definition}

The integrity of an aluminum beverage or food can depends on a polymeric coating (lacquer) that protects the can from a chemical environment containing highly aggressive 
solutions. Without this protective coating, cans would rapidly fail by corrosion. Epoxy resins are used as a protective coating in metal-based food and beverage cans [2].

Bisphenol-A (BPA) is the common name for 2,2-(4,4'-dihydroxydiphenyl) propane, 4,4'-isopropylidenediphenol, or 2,2'-bis(4-hydroxyphenyl)propane [2]. It is used as an intermediate in the production of epoxy resins which are used in the internal coating for food and beverage cans to protect the food from direct contact with metal. BPA can migrate from cans with epoxy coatings into foods, especially at elevated temperatures (for example, for hot-fill or heat-processed canned foods). BPA is one of the 23,000 chemical substances on the CEPA (Canadian Environmental Protection Act) Domestic Substance List (DSL) identified for further evaluation under government of Canada's Chemical Management Plan (CMP) [2].

BPA-coatings prevent both corrosion of the can and contamination of food and beverages with dissolved metals. It plays an important role in preserving the quality and safety of the canned food. Plastics and resins containing Bisphenol-A can also be used in a range of other products including [2]:

- medical devices (e.g. blood oxygenators, incubators, and respiratory devices);

- dental sealants;

- sporting and safety equipment (e.g. hockey helmets);

- electrical (e.g. alarm devices, mobile phone housings, and computers); and

- automotive parts (e.g. headlights, bumpers, and inside lights).

Bisphenol-A is not found naturally in the environment. Bisphenol-A, along with approximately 200 other chemicals, was identified as a high priority under the Government's Chemicals Management Plan; it was identified as a chemical that could 
affect reproduction. Health Canada analyzed the scientific literature, as well as recent assessments by other organizations, and confirmed that the potential health effects were reproductive and developmental [2].

The risk assessment performed by Health Canada confirms that exposure levels are below those that could cause health effects. However, due to the uncertainty raised in some studies relating to the potential effects of low levels of Bisphenol-A, the Government of Canada is taking action to enhance the protection against BPA [2].

Besides the effects of exposure to BPA, Environment Canada has identified concerns about the levels of Bisphenol-A that are being released to the environment. One of the assessments shows that there is the potential for long-term adverse effects to organisms from Bisphenol-A. In addition, these effects can occur at levels that are currently found in the environment close to point sources, such as municipal wastewater treatment plants [3].

The Government is looking at ways to manage the levels of Bisphenol-A being released to the environment at safe concentrations for fish and other aquatic life. The current scientific evidence indicates that the general public need not be concerned as levels of BPA present in food do not pose a health risk [2].

As a precautionary measure to address the environmental concerns, Environment Canada has issued a regulation which requires manufacturers to produce a pollution prevention plan for lowering the levels of BPA in industrial effluents [3]. The regulation would also require facilities using Bisphenol-A to implement best management practices to ensure that it is handled and disposed of safely. These actions will keep the levels of 
Bisphenol-A being released to the environment at safe concentrations for fish and other aquatic life [3].

Environment Canada will work with municipal and provincial counterparts to monitor Bisphenol-A and is currently studying landfills to increase our understanding of trends and how the life cycle of products containing Bisphenol-A affect the environment. There are still many unanswered questions about Bisphenol-A. Therefore, the Government will begin an aggressive research plan focussed on mothers, the fetus, newborns and infants as well as other areas of potential harmful effects, such as prostate/breast cancer, to better define sources of exposure and key points in time when exposures may cause effects [2].

\subsection{Objective of the Research}

Due to the potential harmful effects of Bisphenol-A, new regulations are currently being implemented and Health Canada's Food Directorate continues to place a high priority on the timely evaluation of pre-market submissions for BPA-free can coatings. As these alternative coatings become commercially available, they are anticipated to be gradually used in the packaging of more foods for the general population [2]. This would mean that the coatings currently used to protect food and beverage must be replaced by new formulations. At present there is no fast and convenient method to ratify coatings before or during service, or to test new formulations.

This has initiated a need to develop, test and use new formulations of lacquers, which are as yet untested. The objective of this on-going research is primarily to develop detailed, standardized, accelerated-testing techniques and protocols using 
Electrochemical Impedance Spectroscopy (EIS), along with other electrochemical techniques, to evaluate the durability of Can End Stock (flat stock and converted ends).

EIS is a very powerful technique that can be used to study the structure of very high impedance coatings on materials, and monitor their degradation and failure. Cracks, holes, pores and blisters are all detectable with EIS from earlier in their history than by any non-electrochemical method. EIS will enable evaluation of new coatings in a much shorter timeframe (weeks) than can currently be done with other techniques. The signatures in EIS spectra and polarization experiments that are quantitative indicators of coating failure and corrosion will be identified, and robust laboratory procedures to perform these measurements will be developed.

A future aim of this research will be to extend this technology to develop an online sampling and monitoring system that can be used for quality assurance during the manufacturing process, hence adding to the quality assurance of a product. No such monitor exists at present.

\subsection{Organization of the Thesis}

This thesis is composed of 7 chapters. Chapter 1 introduces the reader to the problem definition and the objectives of the research. Chapter 2 provides an introduction to EIS, followed by an in-depth literature review in which the latest research on using EIS to study coated-aluminum is presented in Chapter 3 .

The testing procedure, equipment, software and corrosion cells utilized to carry out this research are explained in Chapter 4. The preliminary experiments carried out to optimize the systems at hand and to ensure the proper-functioning of the equipment are presented in Chapter 5. 
Chapter 6 presents the experiments performed on the coated-aluminum samples in aerated and de-aerated environments as well as the preliminary tests on the formed canends. The conclusions and recommendations for future experiments are presented in chapter 7 .

\subsection{Methodology}

The main experimental method used in this research is EIS, complemented with potentiodynamic scans. The research was carried out in three main stages:

1) Preliminary experiments: The main objective of this stage was to determine if the EIS systems at hand are functional and produce consistent data. It was important for each EIS instrument to establish the limits within which it gives accurate data. This was followed by EIS testing of kitchen-aluminum foil and high-impedance epoxy polymer to obtain respectively baselines for chemically-active and chemically-inert systems. Early signatures for corrosion and deteriorating coatings were recorded.

2) Experiments in aerated solutions: the effects on the coated-aluminum samples of continuous exposure to different aggressive solutions, containing varied $\mathrm{pH}$ levels and salt concentrations were investigated. The ability of EIS to differentiate between intact and flawed coatings was also tested by investigating deliberately damaged coatings using AC (EIS) and DC (potentiodynamic) methods. The early signatures for corrosion were confirmed. These studies also established the background for the development of an accelerated testing procedure for aluminum lacquers. 
3) Experiments in de-aerated solutions: during prior testing, samples failed in times varying from a few days to a few weeks, while in practice, in the field, similar cans last a very long time before they fail. Since during service conditions, the coated-aluminum samples are exposed to deaerated environments, the effects of such conditions were explored to help understand the prolonged service life of aluminum beverage cans. Once again signatures for corrosion were recorded. 


\section{Chapter 2. Electrochemical Impedance Spectroscopy (EIS)}

\subsection{Introduction}

Electrochemical Impedance Spectroscopy (EIS) is an electrochemical technique with a vast range of applications. Performing a simple search for electrochemical impedance spectroscopy yields over 20,000 publications in many different fields, such as corrosion science, biosensors, battery development, fuel cell development, physical electrochemistry and coating/paint characterization [4]. In the early 1970's, the power of electrochemical impedance spectroscopy as a tool for studying difficult and complicated systems was realized by research electrochemists and materials scientists [5]. The main reason behind its popularity is its ability to provide sensitive, qualitative and quantitative information about the physical and chemical properties and changes in an electrochemical system [4].

EIS involves the application of a small, sinusoidal, alternating current (AC) or voltage to the sample undergoing investigation. The AC perturbation (either voltage or current) is applied over a range of frequencies, and the electrical response of the system is measured and analysed over this range of frequencies [4]. An EIS setup consists of a working electrode (WE), a counter electrode (CE), and a reference electrode (RE). The working electrode, generally the material under investigation, is where the corrosion reaction(s) take place in an electrochemical system. The counter or auxiliary electrode is the electrode in the cell that provides the main current source, and the reference electrode serves as an experimental voltage reference point.

Electrochemical cells can be configured as either a two-electrode, three-electrode or four-electrode electrochemical cell setup. In two-electrode setups, the counter and 
reference electrodes are connected. In all of the experiments described in this thesis, the two-electrode setup was utilized. In the three-electrode setups, the counter and reference electrodes are separated. The potential changes of the working electrode are measured independently of any changes that may occur at the counter electrode in three-electrode setup. Four-electrode setups are relatively uncommon and will not be discussed [6]. Figure 2-1 shows a three-electrode setup for carrying out EIS measurements.

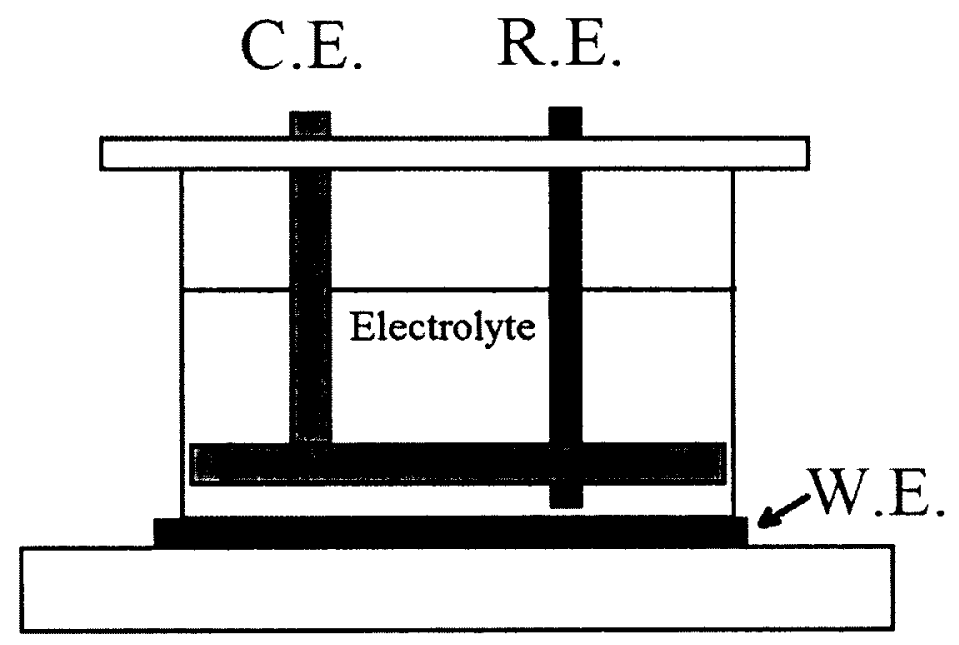

Figure 2-1 Schematic of an electrochemical cell used to carry out EIS measurements on coatings.

\subsection{What is Impedance?}

The concept of electrical resistance is the opposition to the passage of electrical current through an electrical element, defined by Ohm's law as [5]:

$$
R=\frac{V}{l}
$$

where $I$ is the current [A], $V$ is the voltage or the potential $[\mathrm{V}]$, and $R$ is the electrical resistance $[\Omega]$. However, this relationship is limited to one circuit element, the resistor. In the real world, other passive components such as the capacitors, inductors and constant 
phase elements exhibit a much more complex behavior. In place of resistance we use impedance, $Z$, which is a measure of a circuit's tendency to resist (or impede) the flow of an alternating electrical current. The equivalent mathematical expression is [5]:

$$
Z=\frac{V_{a c}}{I_{a c}}
$$

This equation only applies to the time varying, alternating, or $\mathrm{AC}$, components of the current and voltage. The magnitude and time relationships between the voltage and current nearly always depend on the frequency of the alternating current and voltage. Figure 2-2 shows a sine wave voltage applied to an electrochemical cell and the current response [5].

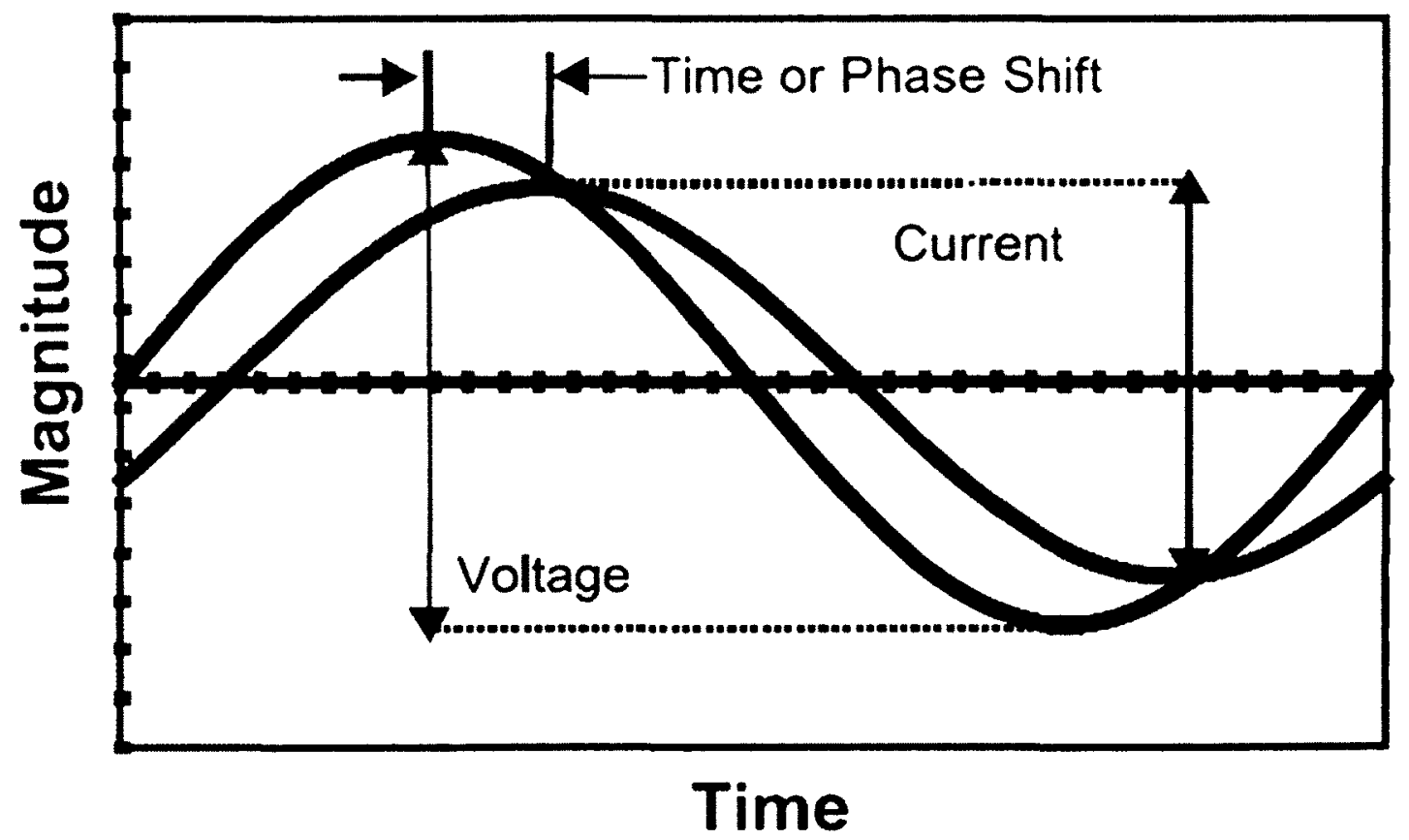

Figure 2-2 A small sinusoidal potential excitation and its current response in an AC system [5].

In Figure 2-2, sine waves representing $\mathrm{AC}$ voltage and $\mathrm{AC}$ current are plotted, but they are shifted in time relative to each other, due to electrical components storing charge 
and energy. The voltage is said to lead the current; i.e., the peak voltage occurs before the peak current. It can also be said that current the lags the voltage. This time shift may be expressed as an angle, the phase angle shift of the current response, or simply the phase angle, $\varphi$. If one cycle $\left(360^{\circ}\right)$ of the sine wave takes $1 \mathrm{~s}$, and the time shift between the current and voltage sine waves is $0.1 \mathrm{~s}$, then the phase angle is $36^{\circ}$. In the case of Figure 2-2, the phase angle would be defined as positive, whereas if the current leads the voltage, the phase angle is considered negative [4].

The magnitude of the impedance $(\Omega)$ of this system can be expressed by taking the ratio of the amplitude of the voltage sine wave (in volts) to that of the current sine wave (in amperes). The magnitude of the impedance, $|Z|$, is sometimes called the modulus of the impedance. To characterize the impedance, $Z$. one must specify its magnitude, $|Z|$, and phase, $\varphi$, as well as the frequency, $f$ (in cycles per second, or Hertz), at which it was measured [5]. These three parameters are often plotted on what is known as a Bode plot, shown in Figure 2-3.

The concept of electrical impedance was introduced by Oliver Heaviside in the 1880 's, after which it was developed in terms of vector diagrams and complex representation by A. E. Kennelly and C. P. Steinmetz [7].

An AC system can be described in terms of complex arithmetical functions. In this representation, the excitation potential and the response current are given by Eq.2-3 and Eq.2-4, respectively [8].

$$
\begin{gathered}
V_{a c}=V_{t}=V_{0} e^{j \omega t} \\
I_{t}=I_{0} e^{j \omega t-j \varphi}
\end{gathered}
$$


where $V_{t}$ is the potential at time $t, I_{t}$ is the current at time $t, V_{0}$ is the amplitude of the voltage, $I_{0}$ is the amplitude of the current, $\varphi$ is the phase shift in the current, $j=\sqrt{-1}$, and $\omega$ is defined in Eq.2-5.as

$$
\omega=2 \pi f
$$

Using Ohm's law, the impedance can be represented as a complex function [4]:

$$
Z=\frac{V_{t}}{I_{t}}=\frac{V_{0} e^{j \omega t}}{I_{0} e^{j \omega t-j \varphi}}=Z_{0} e^{j \varphi}
$$

Applying Euler's relationship [8]:

$$
e^{j \varphi}=\cos (\varphi)+j \sin (\varphi)
$$

The following relationship for impedance can be obtained.

$$
Z=Z_{0}(\cos (\varphi)+j \sin (\varphi))
$$

The impedance is now resolved into real and imaginary components [7],

$$
\begin{aligned}
& Z_{\text {real }}=Z^{\prime}=Z_{0} \cos (\varphi) \\
& Z_{\text {img }}=Z^{\prime \prime}=Z_{0} \sin (\varphi)
\end{aligned}
$$

With a phase angle

$$
\varphi=\tan ^{-1} \frac{Z^{\prime \prime}}{Z^{\prime}}
$$

And a modulus

$$
|Z|=\left[\left(Z^{\prime}\right)^{2}+\left(Z^{\prime \prime}\right)^{2}\right]^{1 / 2}
$$

\subsection{EIS Output}

Three parameters, the magnitude $|Z|$, and phase angle $\varphi$, as well as the frequency $f$ are needed to characterize the impedance of a system. These parameters are plotted on what is known as a Bode plot, shown in Figure 2-3. For the experiments reported here, the 
frequency can range from more than $100,000 \mathrm{~Hz}$ down to $0.001 \mathrm{~Hz}$ or less. Therefore, the frequency axis (the horizontal axis) is plotted logarithmically. Since $|Z|$ can also change by a factor of a million or more in these studies, the $|\mathrm{Z}|$-axis (the vertical axis) is also plotted logarithmically. Bode plots are often used to display EIS data [5].
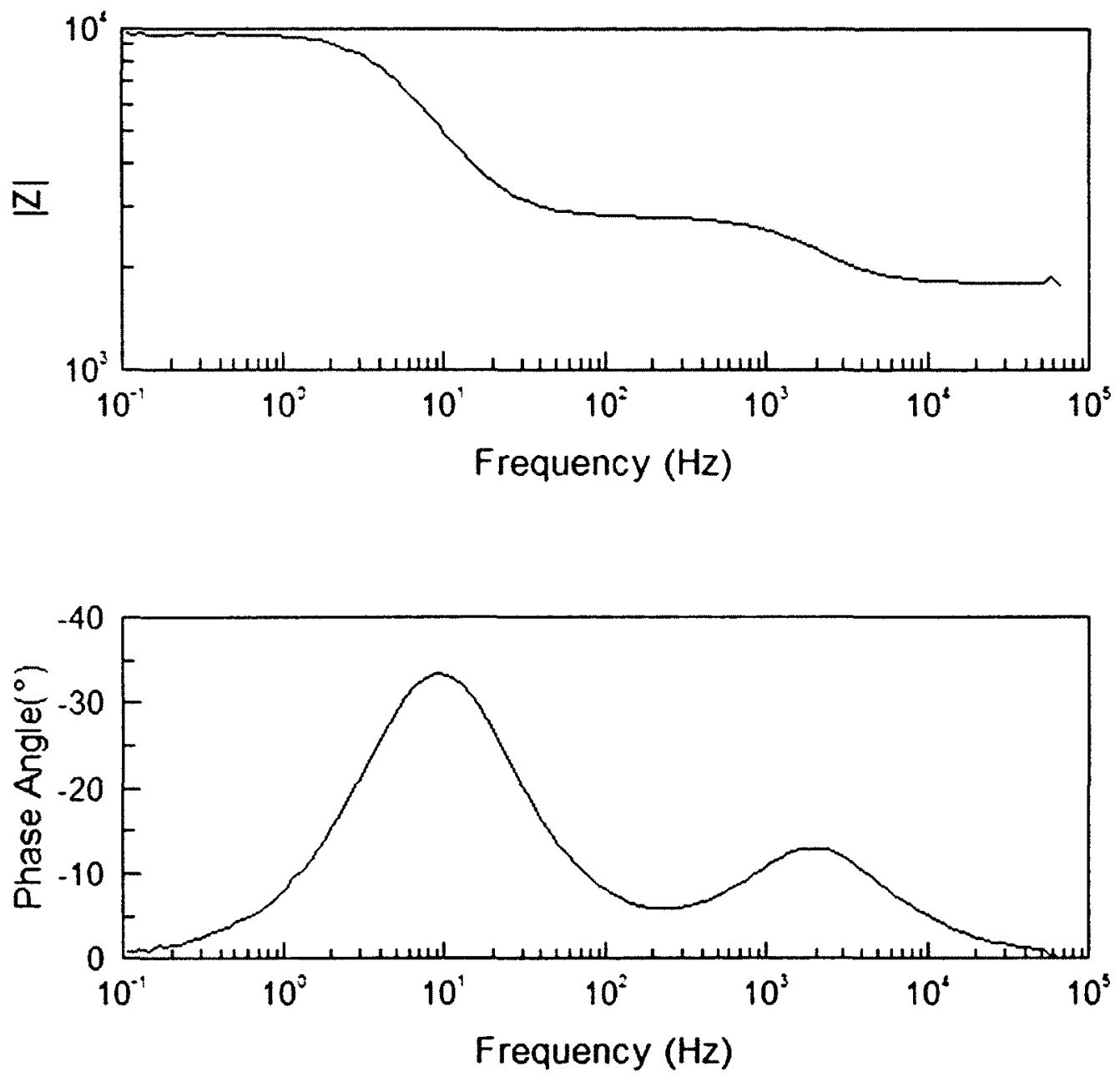

Figure 2-3 Typical Bode plot of an EIS scan.

Other representations for EIS data include the vector representation (polar coordinates) and the complex plane representation known as the Nyquist plot. In the vector representation, Figure $2-4$, the length of the vector is $|Z|$ and the rotation of the vector is the phase angle $(\varphi)$. 


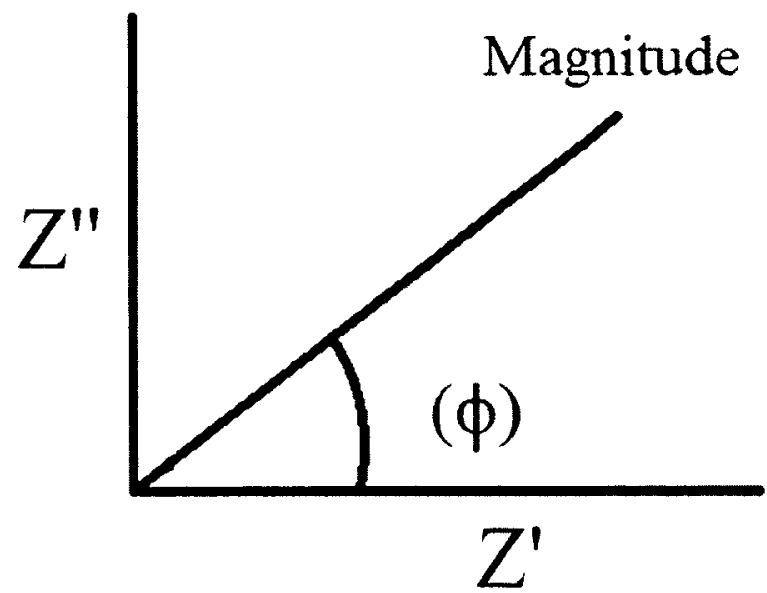

Figure 2-4 Vector representation of EIS data.

In the Nyquist representation, Figure $2-5, Z_{\text {reat }}\left(Z^{\prime}\right)$ is on the horizontal axis and $Z_{i m g}\left(Z^{\prime \prime}\right)$ on the vertical axis. In this thesis, mainly the Bode-plot representation (e.g. Figure 2-3) is utilized. 


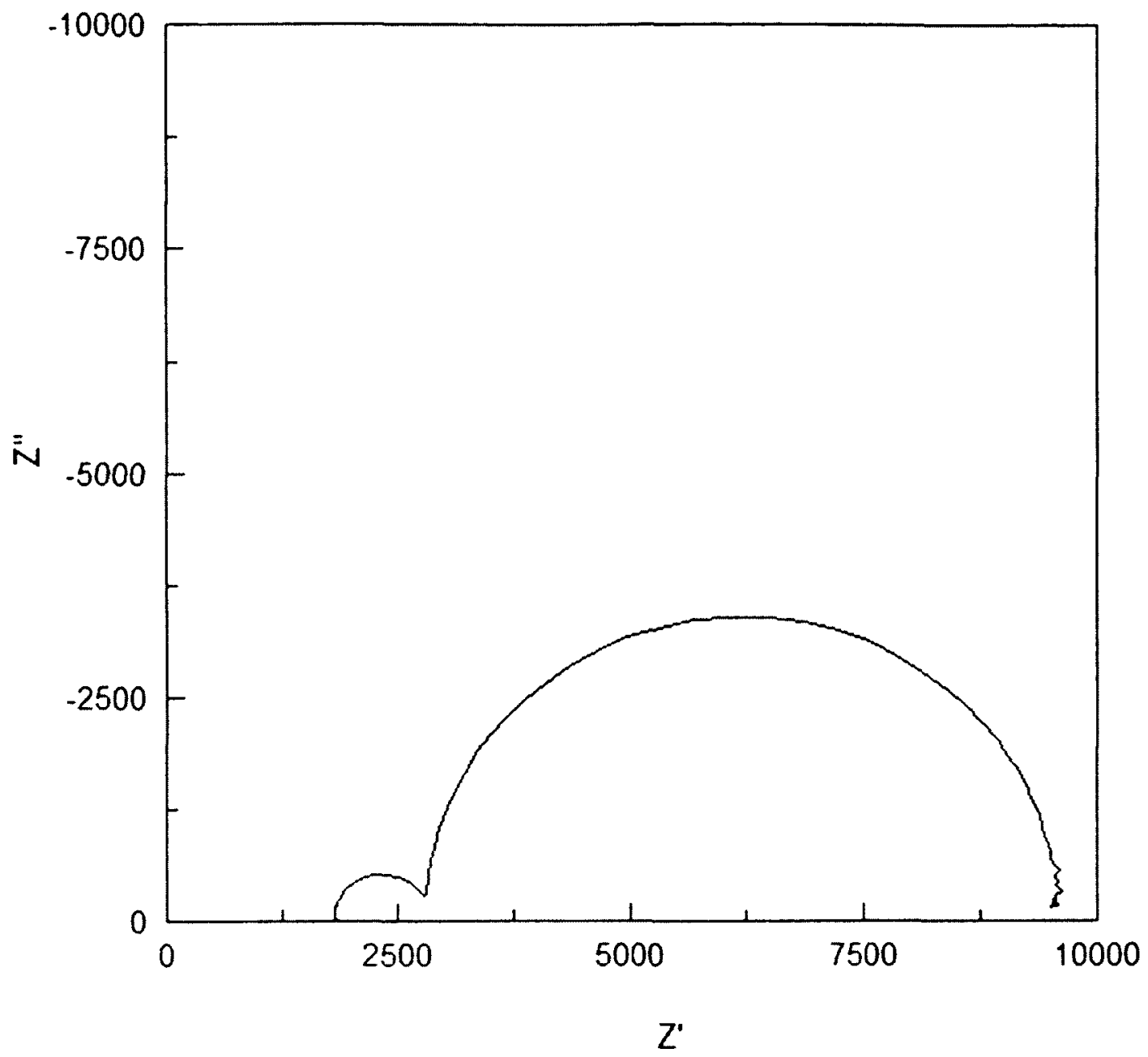

Figure 2-5 Nyquist representation of EIS data shown in Figure 2-3.

\subsection{Equivalent Circuit Modelling}

Electrochemical systems such as coated surfaces or corroding metals often behave like simple electrical circuits [7] so EIS data are commonly analyzed by fitting them to an equivalent electrical-circuit model. This procedure is known as Equivalent Circuit Analysis (ECA) [4]. Provided that the model is physically representative of the actual measured system, the variation of the model components can be tracked with respect to time so that changes in the physical system can be quantified as a function of time [5]. 
The measured data are fitted to an assumed model to obtain the magnitudes of appropriate circuit elements. It is possible to derive quantitative information on the thickness, porosity and integrity of a coating by performing such analysis [4].

It is important to first understand the basic passive circuit elements and their combinations before trying to understand the proposed models for coated metals. The basic circuit elements, relevant to this thesis, are the resistor, capacitor, inductor and constant phase element.

If a sine wave voltage is applied across an ideal resistor, the current will also be a sine wave in phase with the voltage with no time lag; it has a phase angle of $0^{\circ}$. The current through a resistor reacts instantaneously to any change in the voltage applied across the resistor. For an ideal resistor, this is true at all frequencies. Also, the amplitude of the current sine wave does not depend upon the frequency; it depends only on the Resistance $\mathrm{R}$ (in $\Omega$ ) and the amplitude of the voltage sine wave. The impedance of a resistor is straightforward to write as a complex number. In complex coordinates the phase angle is always $0^{\circ}$, the "vector" always lies on the real axis and the Y (imaginary) component is always zero [5].

$$
\begin{aligned}
Z_{\text {resistor }} & =Z_{\text {real }}+j Z_{i m g} \\
& =R+j 0 \\
& =R
\end{aligned}
$$

Since the impedance of a resistor is independent of frequency, the Bode magnitude plot is just a horizontal straight line (slope equals 0 ) and the Bode phase plot is always zero. An example of a resistance in electrochemistry is the resistance of the 
electrolyte in the electrochemical cell. The resistance of a column of electrolyte can be calculated by [5]:

$$
R=\frac{\rho L}{A}
$$

where $L$ is the length of the column, $A$ is the cross-sectional area, and $\rho$ is the resistivity of the electrolyte $(\Omega-\mathrm{cm})$.

Another simple, yet useful, electric circuit element is the capacitor. Sandwiching a piece of non-conducting plastic or polymer (called a dielectric) between two metallic conducting plates makes a capacitor. A capacitor is a passive circuit element that can store energy. The process of storing energy in the capacitor is known as "charging" and involves the separation of electric charges, which build up on each plate, with equal magnitude but opposite polarity [8].

Unlike the resistor, the current is $-90^{\circ}$ out-of-phase with the voltage. A sine-wave voltage waveform leads to a cosine-current waveform. The current depends on the frequency and reaches a maximum when the voltage is changing the fastest; as it crosses through zero. The higher the frequency, the more rapidly the voltage changes, and the higher is the magnitude of the current. Since the impedance is the ratio of voltage to current, a larger current at higher frequencies leads to a smaller impedance. At zero frequency (DC), the current is zero since there is a non-conductor between the plates. As you approach zero frequency, current approaches zero and the impedance, $Z$, becomes infinitely large. The impedance of a capacitor can be expressed as [5]: 


$$
\begin{gathered}
Z_{\text {capacitor }}=Z_{\text {real }}+j Z_{\text {img }} \\
=0+j\left[\frac{-1}{2 \pi f C}\right] \\
=j\left[\frac{-1}{2 \pi f C}\right]=\frac{1}{j \omega C}
\end{gathered}
$$

Where $Z_{\text {real }}$ is the real part of the impedance and $Z_{\text {ing }}$ is the imaginary part.

Since the impedance of a capacitor varies with the inverse of the frequency, the Bode magnitude plot for a pure capacitor is a straight line with a slope of -1 , and the Bode phase-angle plot is a horizontal line at $-90^{\circ}$. In the case of a metal coated with an impervious coating and immersed in an electrolyte, the metal substrate forms one plate of the capacitor and the conducting electrolyte forms the other plate. The impervious coating separating the two is the dielectric of the capacitor. The capacitance can be calculated from the dimensions of the capacitor, and the nature of the material separating the plates using [8]:

$$
C=\frac{\varepsilon \varepsilon_{0} A}{d}
$$

Where $C$ is the capacitance $(\mathrm{F}), A$ is the plate area, $\varepsilon$ is the dielectric constant of material separating the plates, and $d$ is the thickness of the material; $\varepsilon_{0}$ is $8.85 \times 10^{-14} \mathrm{~F} / \mathrm{cm}$. For a $5 \mu \mathrm{m}$ coating, capacitances can be on the order of $\sim 1 \mathrm{nF} / \mathrm{cm}^{2}[5]$.

An inductor, $L$, is another passive circuit element, traits of which will be observed in this thesis. Unlike capacitors, the voltage leads the current in inductors, leading to a phase-angle of $+90^{\circ}$ in a Bode plot. The impedance of an inductor is given by: 


$$
\begin{aligned}
Z_{\text {inductor }} & =Z_{\text {real }}+j Z_{\text {img }} \\
= & 0+j \omega L \\
& =j \omega L
\end{aligned}
$$

Since the impedance of an inductor varies linearly with frequency, the Bode magnitude plot for a pure inductor is a straight line with a slope of +1 on a log-log plot.

During the application of coatings, non-uniform distribution of the coatings can cause surface roughness and internal inhomogeneities, which can lead to inhomogeneous distributions of the current. In such cases, ideal capacitors are not able to model experimental data, and EIS data are commonly modelled using constant-phase elements (CPE) instead of pure capacitors [9].

A constant phase element (CPE) is another passive circuit element (not a resistor, capacitor or inductor). It can sometimes be viewed as a non-ideal capacitor, which represents a real-world inhomogeneous system. The impedance of a CPE is given by [8]:

$$
Z=\frac{1}{Q(j \omega)^{n}}
$$

When the exponent, $n$, is equal to 1 , the CPE is equivalent to a perfect capacitor $(Q=C)$, when it is zero it is equivalent to a resistor, and when it is -1 it is equivalent to an inductor. The phase angle of a CPE is constant, and is given by [8]:

$$
\varphi_{C P E}=\frac{-n \pi}{2}
$$

A CPE becomes a Warburg element when ionic diffusion limits the current flow. In this case, $n$ has a value of 0.5 . It is commonly used when the diffusion of species through the pores of the coating or corrosion products controls the corrosion rate 
producing a distinctive $45^{\circ}$ tail in the low-frequency region of the Nyquist plot [9]. For real systems containing an imperfect layer of material, $\mathrm{n}$ is usually between 0.5 and 1 [8].

Different passive circuit elements can be combined together in several ways to develop equivalent circuits that are representative of the electrochemical system under investigation. Different equivalent electric circuits can be constructed from elements in series, Figure 2-6, or in parallel, Figure 2-7, or from combinations of series and parallel elements.

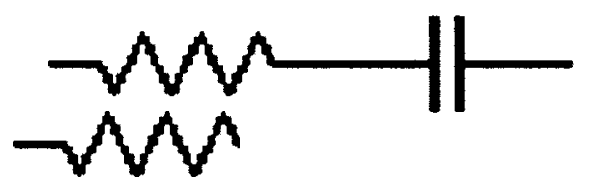

Figure 2-6 A resistor and a capacitor in series

For this system, which consists of a resistor and capacitor in series, the impedance is calculated as:

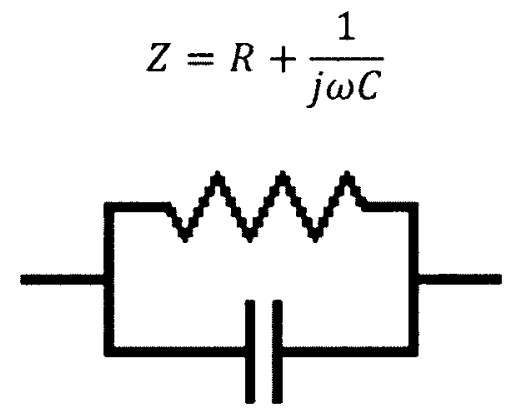

Figure 2-7 A resistor and a capacitor in parallel

For a system which consists of a resistor and capacitor in parallel, the impedance is calculated as:

$$
\frac{1}{Z}=\frac{1}{R}+\frac{1}{\frac{1}{j \omega C}}
$$




$$
Z=\frac{R}{1+R j \omega C}=\frac{R}{1+(R \omega C)^{2}}-j \frac{R \omega C}{1+(R \omega C)^{2}}
$$

\subsection{The Use of EIS to Study Barrier Coatings}

Corrosion is an electrochemical process. If the flow of electrons or ions is stopped, corrosion will be prevented. Therefore, the electrical properties of the coating are germane to the ability of the coating to be an effective protection from corrosion; a good coating acts as a perfect electrical and chemical barrier to protect the underlying substrate. It prevents any chemical species in the environment from passing through to the vulnerable substrate $[10]$, thus preventing electrochemical corrosion. Assuming that the chemical barrier properties of the coatings are indeed related to the electrical barrier properties, EIS can be used to establish quantitatively the integrity and performance of a barrier coating.

A perfect barrier coating behaves like a perfect capacitor [8]. Charge builds up upon its surface but cannot pass through it. On the other hand, a bad barrier coating that contains surface defects such as scratches, cracks, holes or pores, becomes conductive when exposed to an aqueous medium, and allows chemically undesirable liquid or gas to access the vulnerable underlying surface.

The impedance is measured at different frequencies, and the system's response over the desired range of frequencies is monitored. Any changes in the spectra (i.e., $Z_{\text {real, }}$ $Z_{\text {img }},|Z|$ and $\left.\varphi\right)$, over time and in different chemical conditions, are recorded and analysed in terms of underlying degradation mechanisms. The general trend for a degrading coating, as seen in Figure 2-8, is a continual decrease in the impedance and a phase angle approaching $0^{\circ}$ at higher frequencies. The data shown in Figure 2-8 were 
collected during this present investigation for a coated aluminum sample exposed to $1 \mathrm{M} \mathrm{NaCl}$ with a $\mathrm{pH}$ of 8 for 160 hours.
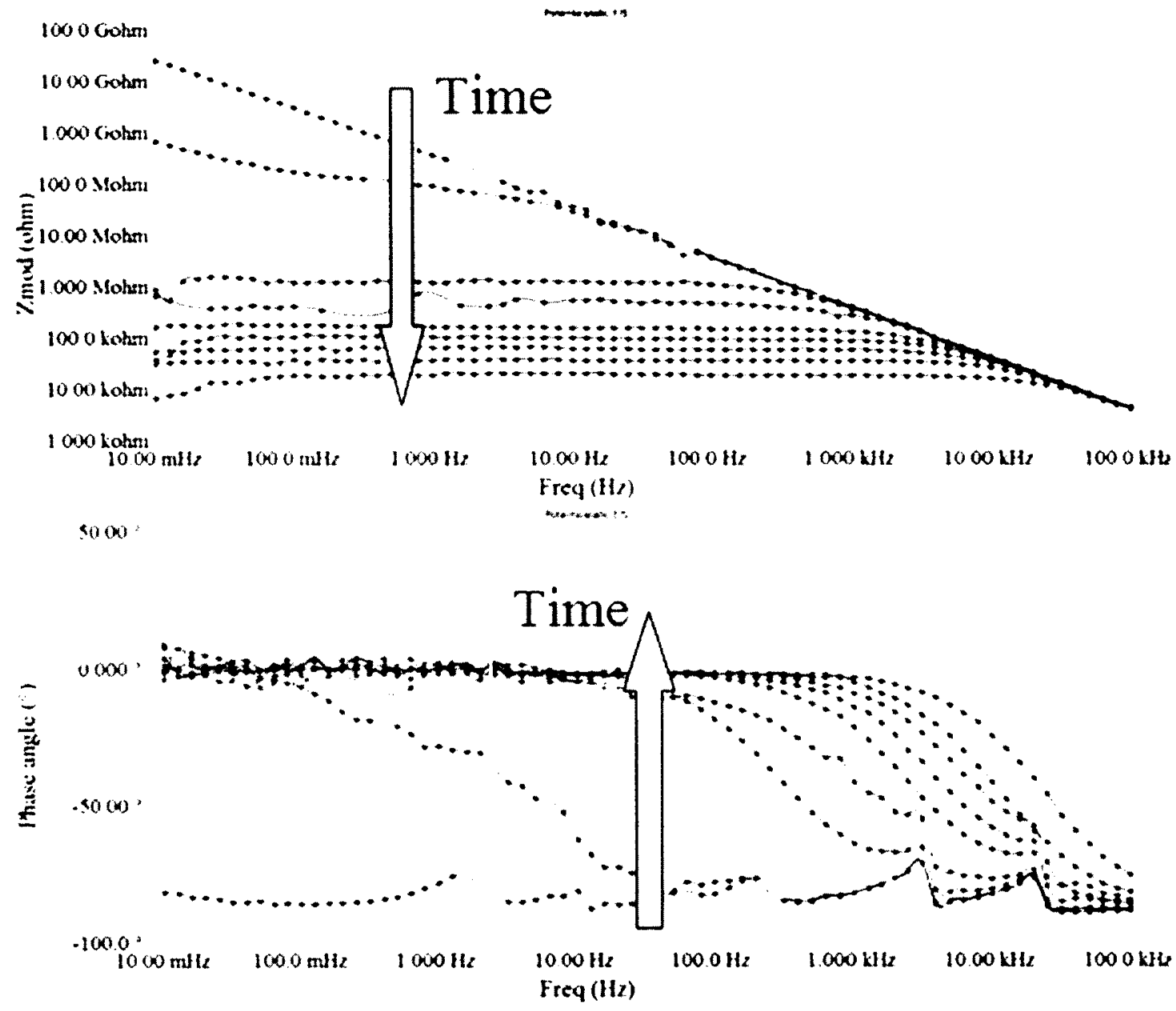

Figure 2-8 EIS data for a coated aluminum sample exposed to $1 \mathrm{M} \mathrm{NaCl}(\mathrm{pH} 8)$ for 160 hours. As penetration of the coating occurs, the impedance at low frequencies continuously drops, changing from a capacitor to a resistor at ever higher frequencies. The phase angle, originally $-90^{\circ}$ across the spectrum, increases from $-90^{\circ}$ to $0^{\circ}$ at ever higher frequencies.

As mentioned earlier in section 2.4 , the data can be fitted to equivalent circuit models to interpret and quantify the physical properties and efficacy of the coating, and can be used to model its deterioration with time. To use such a technique, the following guidelines must be met [11]: 
- All elements of the proposed circuit must have a clear physical meaning and must be associated with physical properties of the system that can generate this kind of electrical response.

- The error between the generated data and the experimental data must be small (as defined by the user/modeller) and not depend on external factors, such as the frequency. The equivalent electrical circuit has to be as simple as possible. If by eliminating an element, the error is still acceptable, then the circuit must be simplified.

One of the simplest equivalent circuits for modeling an intact capacitive coating is the Randles circuit illustrated in Figure 2-9. This electrical circuit can be used to represent a coating or a corroding metal [5].

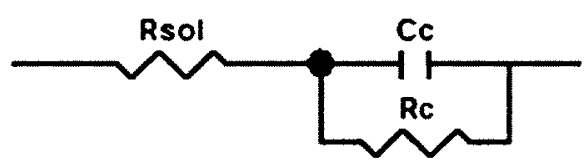

Figure 2-9 Schematic of the Randles circuit

This model consists of a resistor in series with a parallel RC. The resistor $\left(\mathrm{R}_{\mathrm{sol}}\right)$ represents the solution resistance of the electrolyte in the electrochemical cell. The capacitor $\left(\mathrm{C}_{\mathrm{c}}\right)$ is the capacitance of the coating and the resistor $\left(\mathrm{R}_{\mathrm{c}}\right)$ represents the resistance of ionic conduction paths that traverse the coating. This is sometimes referred to as pore resistance. Coating resistances can be quite high, greater than $10^{10} \Omega \mathrm{cm}^{2}$, for a good epoxy coating [12]. The total impedance of this circuit is calculated as follows:

$$
Z_{\text {Randles }}=R_{\text {sol }}+Z_{R C}
$$

Where the impedance of the parallel components $Z_{R C}$ is given by equation 2-22. 


$$
Z_{\text {Randles }}=R_{\text {sol }}+\frac{R_{c}}{1+\left(R_{c} \omega C_{c}\right)^{2}}-j \frac{R_{c} \omega C}{1+\left(R_{c} \omega C_{c}\right)^{2}}
$$

While the impedance of a resistor is constant, the impedance of a capacitor is frequency dependent. As the frequency increases, the impedance due to $C_{c}$ decreases. At the high frequency region, the impedance of the capacitor, at some frequency, will become smaller than $R_{\text {sol }}$. Thus, the high frequency behavior of the Randles circuit is controlled almost entirely by $R_{\text {sol }}$.

As the frequency decreases, the impedance due to $C_{c}$ increases. Since $C_{c}$ is in parallel with $R_{c}$, when the capacitor is small it can effectively remove the resistor from the circuit. Eventually, at some frequency, the impedance of $C_{c}$ will be higher than $R_{c}$ and the capacitor ultimately acts as an open circuit and is effectively removed from the circuit. The impedance of the Randles circuit is then the combined resistance values of the two series resistors, $R_{\text {sol }}$ and $R_{c}$. Thus, in both the high and low frequency regions, the Randles circuit behaves primarily as a resistor, so that the imaginary component is very small, the phase angle is close to $0^{\circ}$, and the impedance does not change with frequency.

At intermediate frequencies, the capacitor's impedance is important and the cell acts like a capacitor. The imaginary component becomes significant, the phase angle approaches $-90^{\circ}$ and the cell impedance becomes linearly frequency dependent (on a log$\log$ scale).

Generally, when a protective coating is first immersed in an electrolyte, the impedance produced by the coating capacitance will be smaller than the solution resistance at the high frequency end, and greater than the pore or coating resistance at the low frequency end of the spectrum. Over a large frequency range, the Bode impedance plot will be a straight line with a slope of -1 and the phase angle of the system will be 
$-90^{\circ}$ as in Figure 2-10. The resistive behaviour of the Randles circuit at the extremities of the frequency spectrum may or may not be observed in the Bode plot depending on the measuring capabilities of the EIS system at hand.

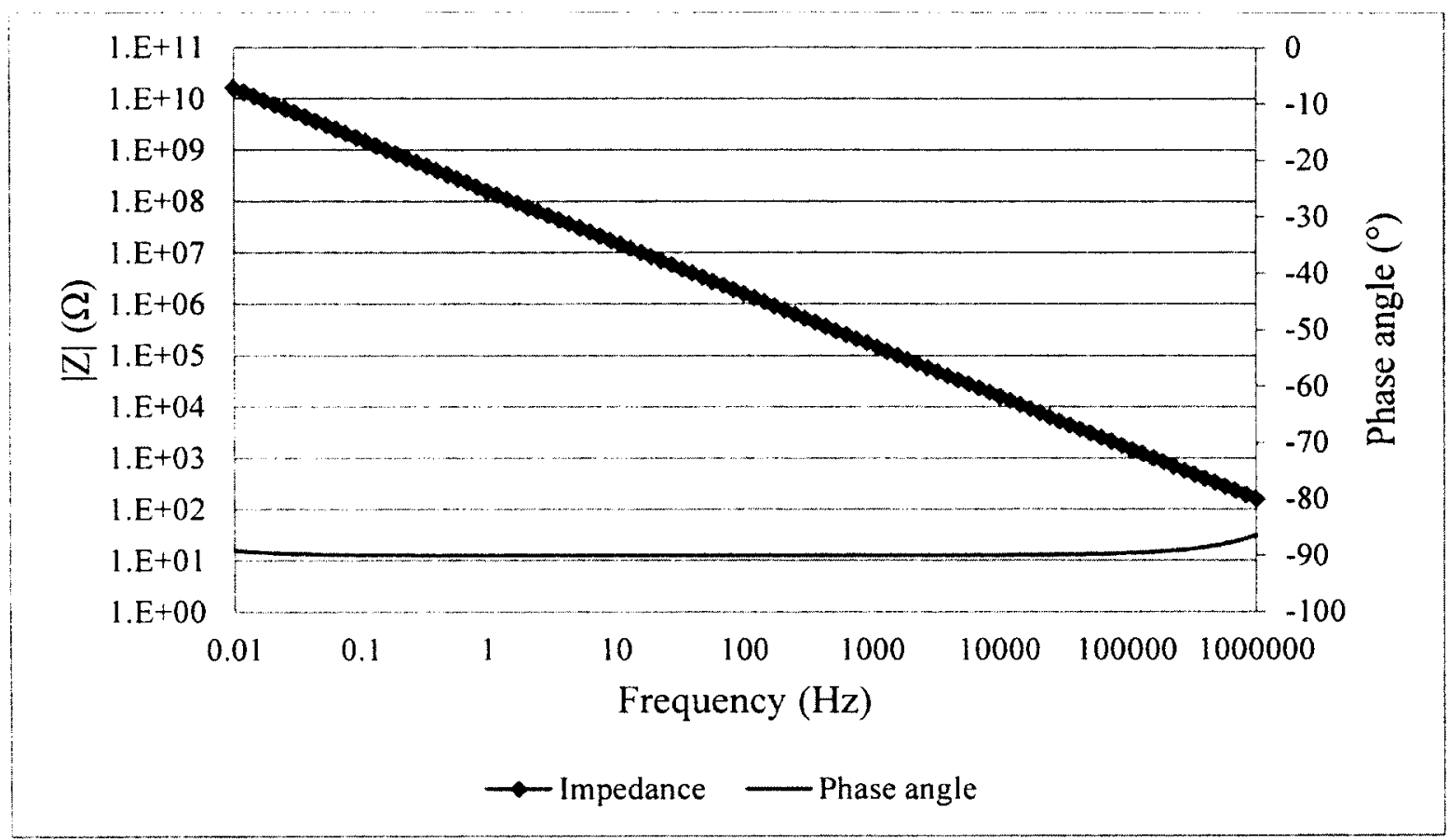

Figure 2-10 Simulated Bode plot for an intact coating represented with a Randles circuit with $R_{\text {sol }}=10 \Omega, C_{c}=1 \mathrm{nF}$ and $R_{c}=1 \mathrm{~T} \Omega$.

In a Nyquist plot, a capacitive element will appear as a vertical line. By introducing $R_{c}$ and $R_{\text {sol }}$, the Nyquist plot will start to draw a semi-circle as seen in Figure 2-11; the Nyquist curve intercepts the real-axis at the value of $\mathrm{R}_{\text {sol }}$ near the origin. 


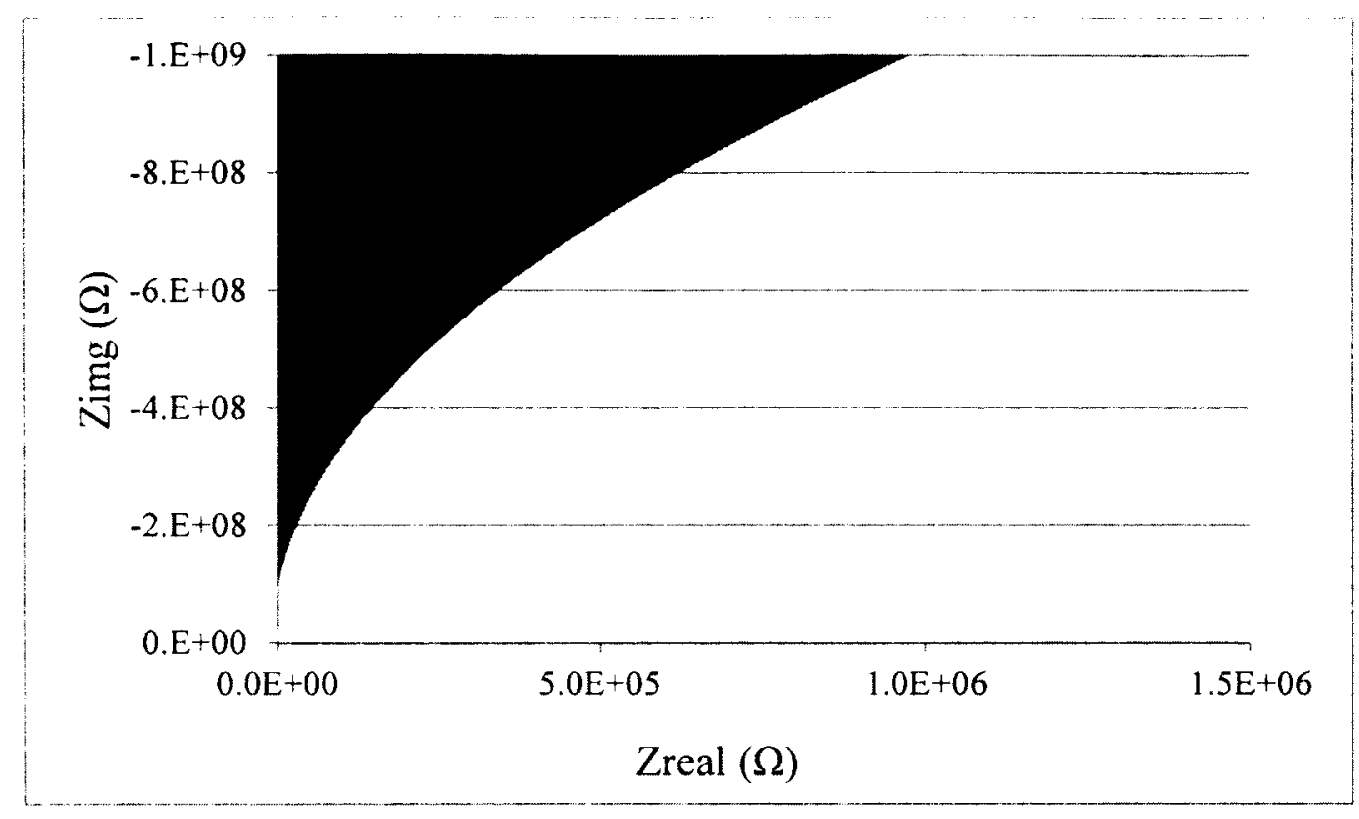

Figure 2-11 Simulated Nyquist plot for an intact coating represented with a Randles circuit with $R_{\text {sol }}=10 \Omega, C_{c}=1 n F$ and $R_{c}=1 T \Omega$.

For some polymers, but not all, at some undefined time, the ionic species from the electrolyte can diffuse into the microporosity within the polymer coating, resulting in a drop in the coating or pore resistance, so that a measureable pore resistance now comes into play. As shown in Figure 2-12, at the low end of the frequency spectrum, the impedance plot is horizontal and the phase angle approaches $0^{\circ}$. At this instant, the semicircle is more apparent in the Nyquist plot, Figure 2-13; the Nyquist curve intercepts the real-axis at the value of $R_{\text {sol }}$ and $R_{c}+R_{\text {sol }}$. 


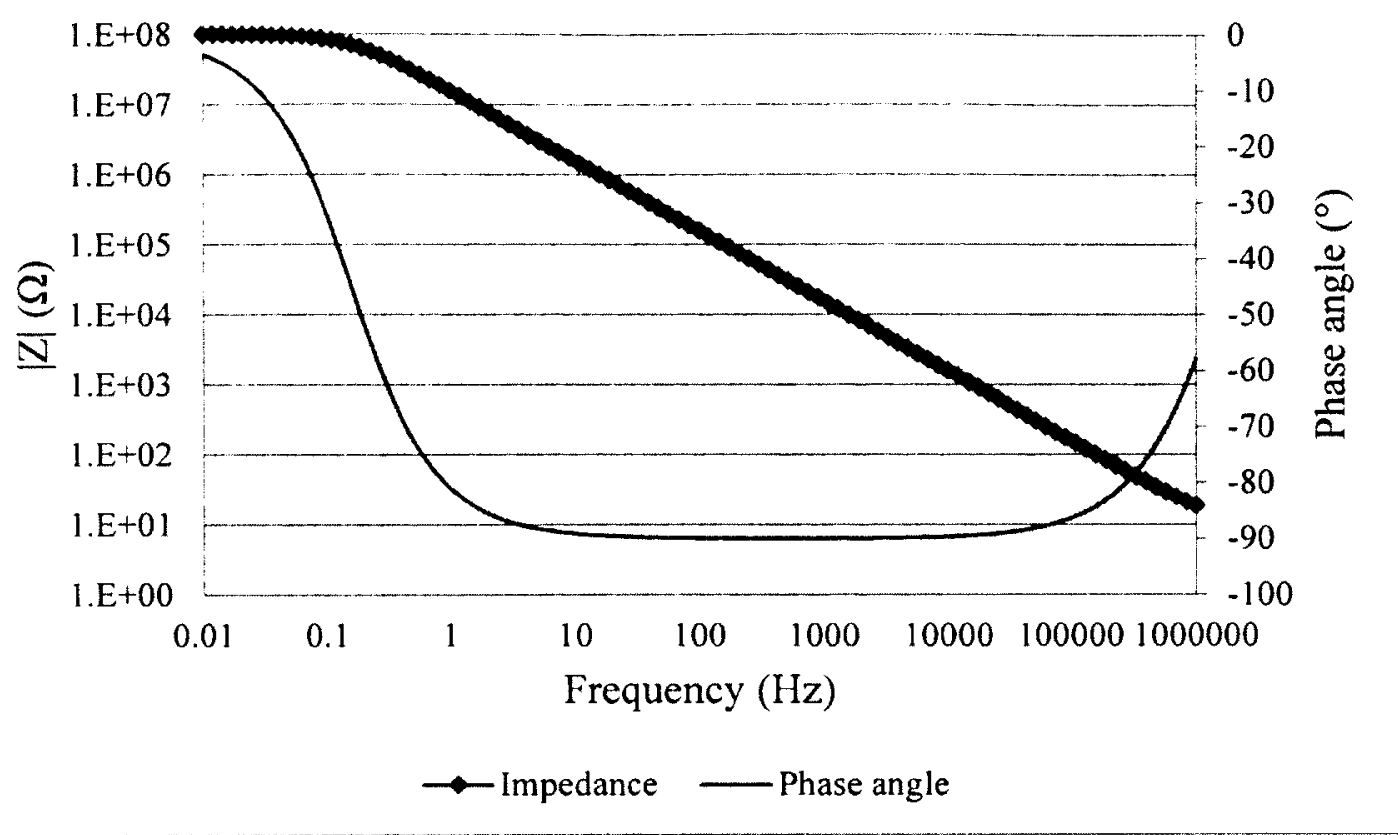

Figure 2-12 Simulated Bode plot for a coating with solution penetrating the pores represented with a Randles circuit with $R_{\text {sol }}=10 \Omega, C_{c}=10 \mathrm{nF}$ and $\mathbf{R}_{\mathrm{c}}=\mathbf{1 0 0} \mathrm{M} \Omega$.

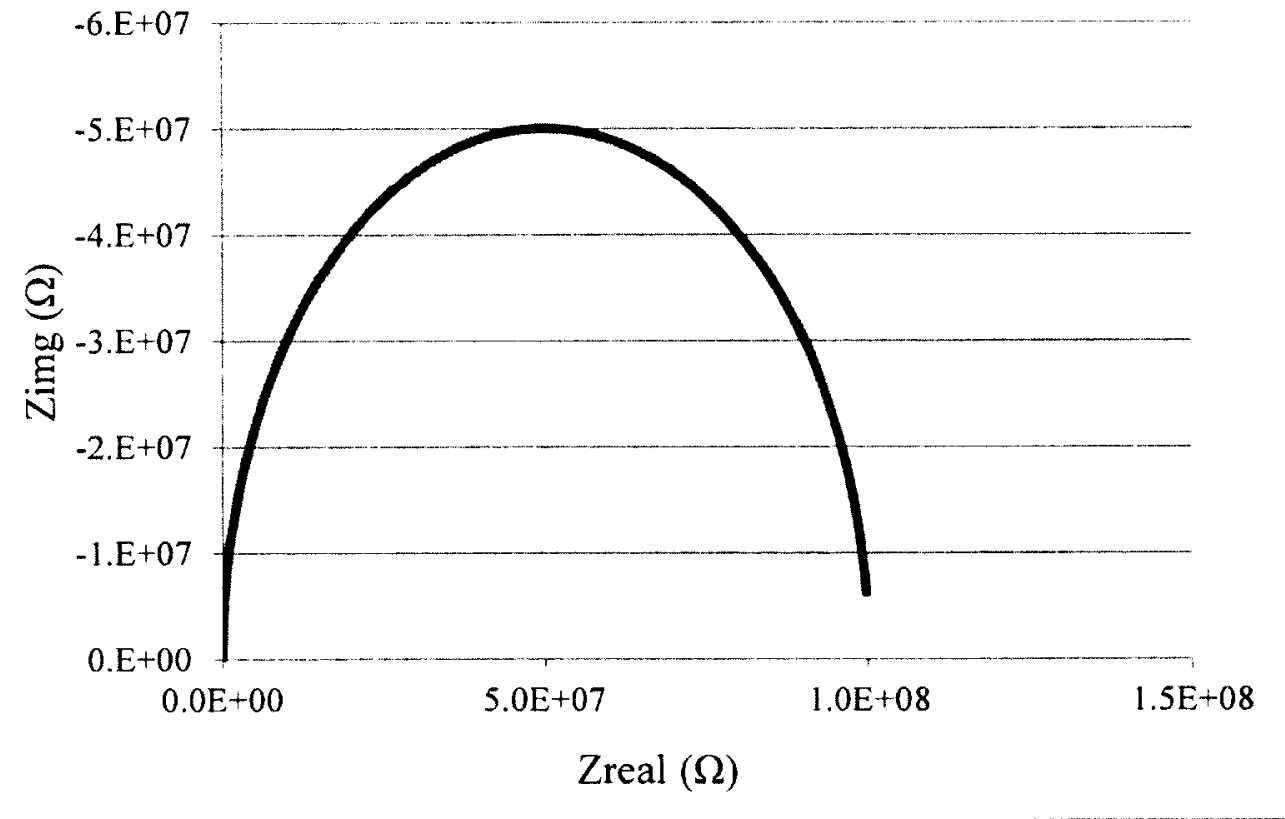

Figure 2-13 Simulated Nyquist plot for a coating with solution penetrating the pores represented with a Randles circuit with $R_{\text {sol }}=10 \Omega, C_{c}=10 \mathrm{nF}$ and $\mathrm{R}_{\mathrm{c}}=100 \mathrm{M} \Omega$. 
As coatings degrade, they may show an increasingly complicated behaviour [13]. A model for a failed coating in which ions and solution come into contact with the metal substrate is illustrated in Figure 2-14 [13].

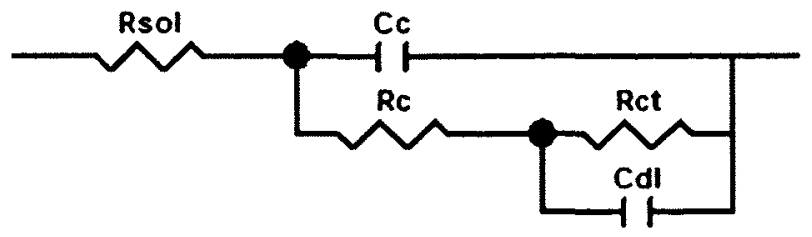

Figure 2-14 Electrical circuit model for a failed coating.

In one of the interpretations of this model, an assumption is made that the coating has partially delaminated from the metal substrate and the delaminated area is filled with solution. This electrolyte solution can have very different chemistry than the bulk solution outside the coating. The interface between this pocket of solution and the bare metal is modeled as a double layer capacitance, $C_{d l}$, in parallel with a kinetically controlled charge transfer reaction element represented by the charge transfer resistance $R_{\mathrm{ct}}$. It is sometimes also represented as a polarization resistance $R_{p}$, an element representing the resistive corrosion product layer on the metal plate [8]. Simulation of the electrical circuit in Figure 2-14 is shown in Figure 2-15 and Figure 2-16. In the Nyquist plot, a second semicircle starts to appear. The first semicircle intercepts the real-axis, near the origin, at the value of the $R_{\text {sol }}$ and ends at a value of $R_{c}+R_{s o l}$, where the second semicircle starts. The second semicircle ends at a value of $R_{c}+R_{s o l}+R_{c t}$. 


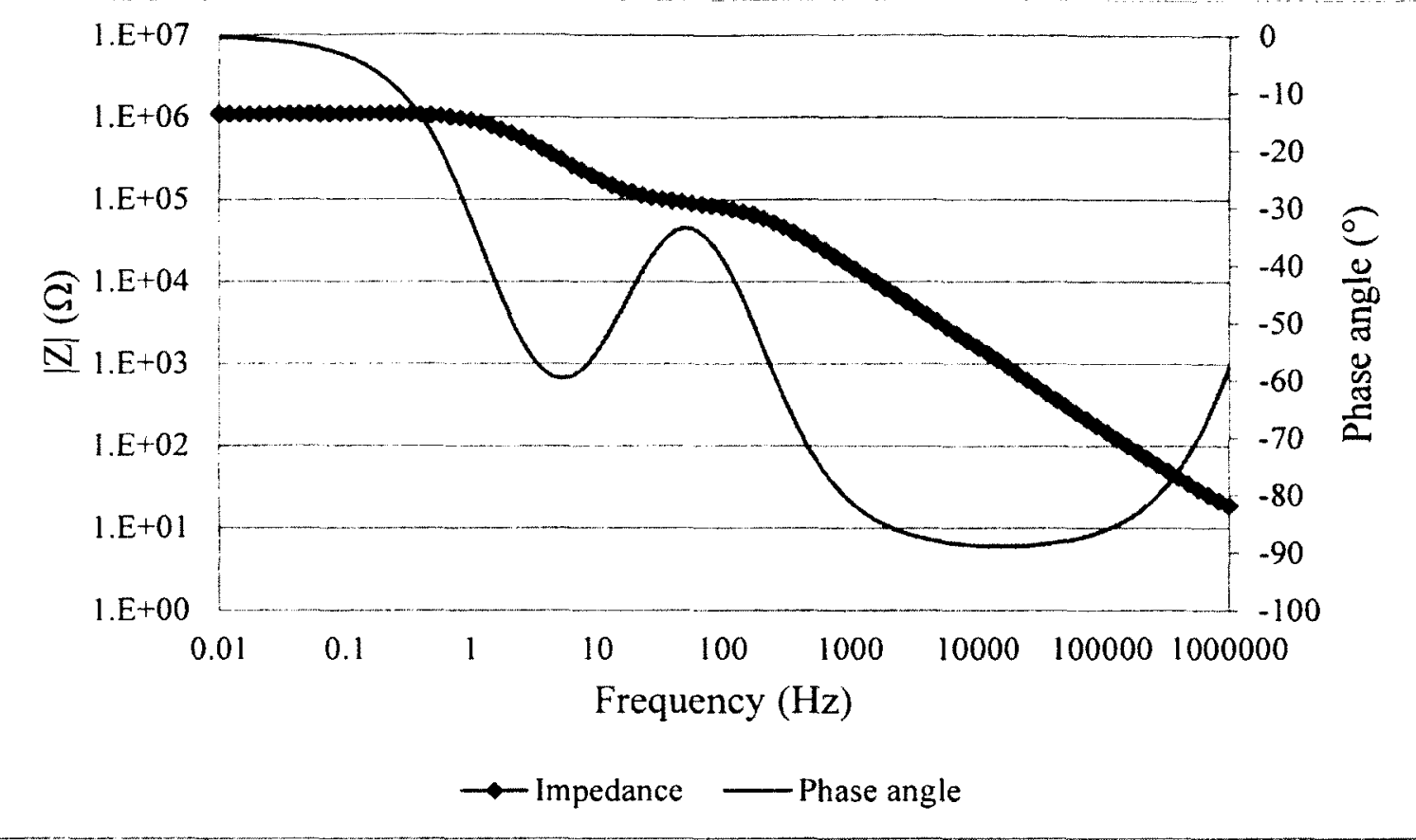

Figure 2-15 Simulated Bode plot for a coating with a freely corroding substrate represented by the circuit shown in Figure 2-14 with $R_{\text {sol }}=10 \Omega$, $C_{c}=10 \mathrm{nF}, R_{c}=100 \mathrm{k} \Omega, C_{d l}=100 \mathrm{nF}$ and $R_{c t}=1 \mathrm{M} \Omega$.

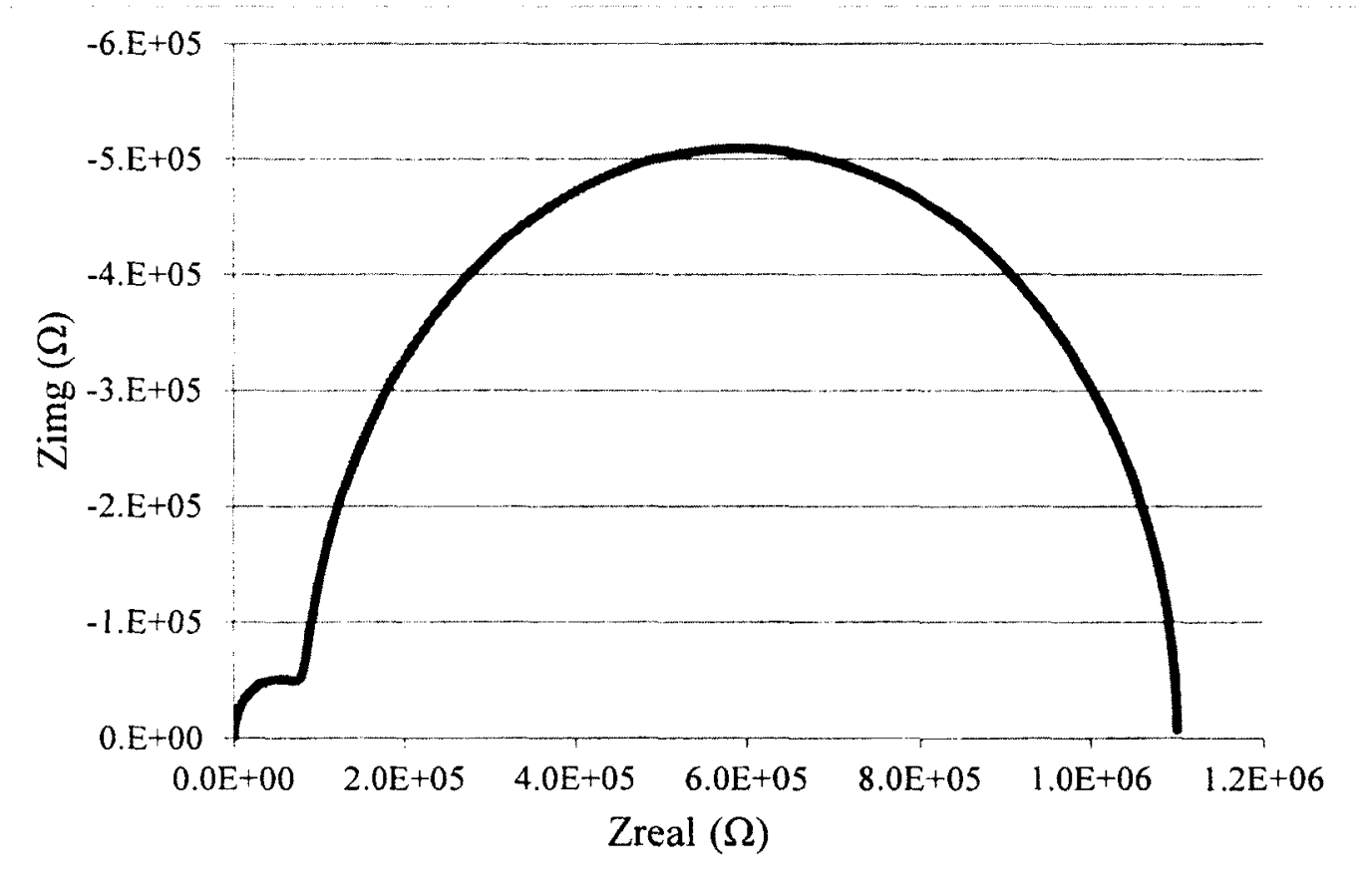

Figure 2-16 Simulated Nyquist plot for a coating with a freely corroding substrate represented by the circuit shown in Figure $2-14$ with $R_{\text {sol }}=10 \Omega$, $C_{c}=10 \mathrm{nF}, R_{c}=100 \mathrm{k} \Omega, C d l=100 \mathrm{nF}$ and $R_{c t}=1 \mathrm{M} \Omega$. 
$R_{c t}$ is inversely proportional to the corrosion rate. A typical charge transfer resistance for a bare metal is $5000 \Omega \mathrm{cm}^{2}$, and the value of $\mathrm{C}_{\mathrm{dl}}$ is usually in the range of $10-40 \mu \mathrm{F} / \mathrm{cm}^{2}$ [12]. This capacitance is much higher than $C_{c}\left(\sim 1 \mathrm{nF} / \mathrm{cm}^{2}\right)$, so the $C_{d l}$ of even a small defect will be apparent in the EIS response. A defect, such as a scratch, that exposes only $0.005 \%$ of the total sample area to the electrolyte has the same capacitance $\left(20 \mu \mathrm{F} / \mathrm{cm}^{2} * 0.005 \%=1 \mathrm{nF} / \mathrm{cm}^{2}\right)$ as the entire intact area of the coating $(1 \mathrm{nF})$. Note that $R_{\mathfrak{c} 1}$ and $C_{d l}$ must be normalized because they are electrode-area dependent [12].

With continued exposure to a corrosive medium and the substrate actively corroding, total failure of the coating will surely happen. Figure 2-17 and Figure 2-18 show the Bode and Nyquist plots of a severely failed coating. In the Nyquist plot, the first semicircle intercepts the real-axis, near the origin, at the value of the $R_{\text {sol }}$ and ends at a value of $R_{c}+R_{\text {sol }}$, where the second semicircle starts. The second semicircle ends at a value of $R_{c}+R_{\text {sol }}+R_{c t}$. 


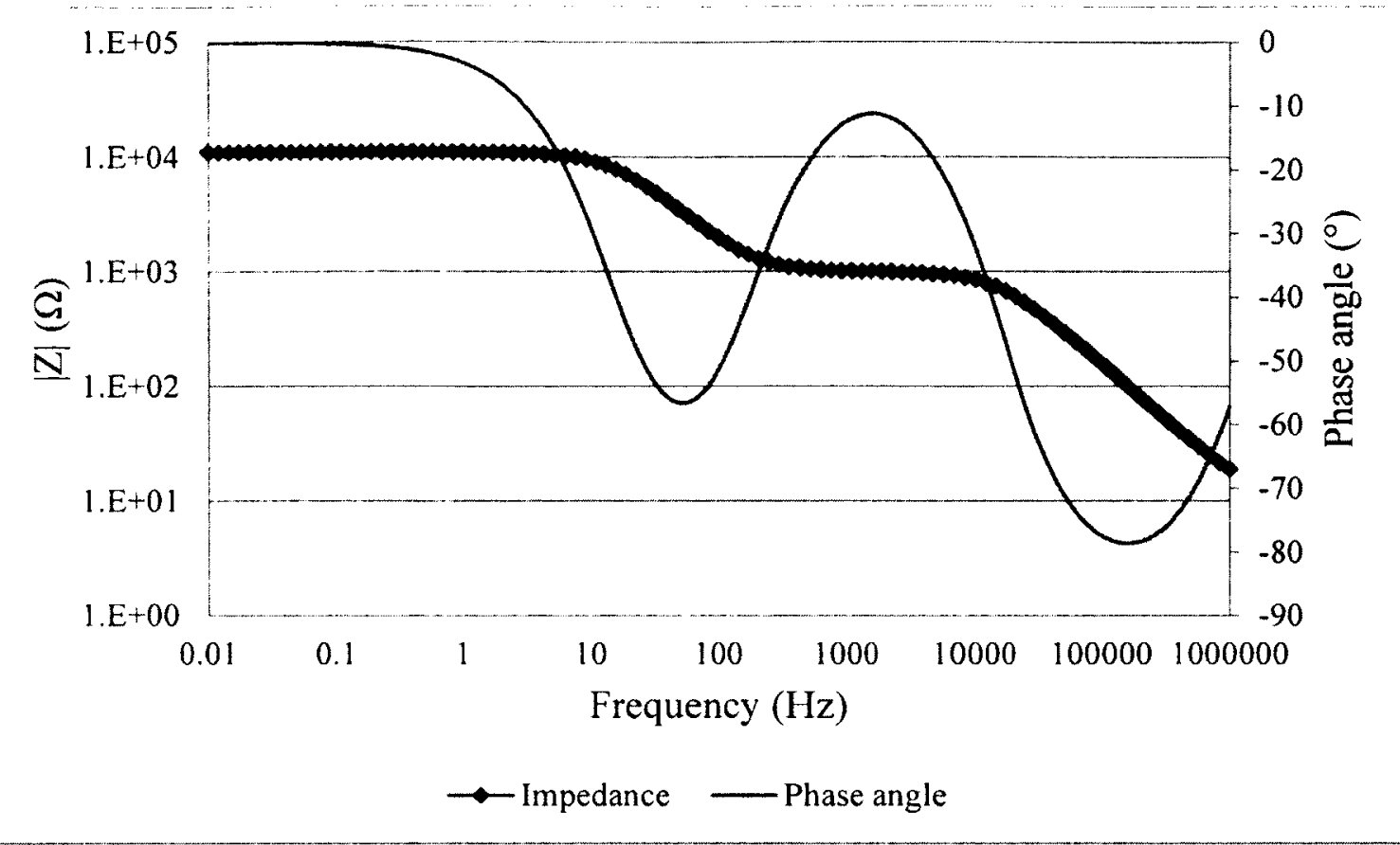

Figure 2-17 Simulated Bode plot for a severely damaged coating represented by the circuit shown in Figure 2-14 with $R_{s 0 l}=10 \Omega, C_{c}=10 \mathrm{nF}, R_{c}=1 \mathrm{k} \Omega$, $C_{\mathrm{dl}}=1 \mu \mathrm{F}$ and $\mathrm{R}_{\mathrm{ct}}=10 \mathrm{k} \Omega$.

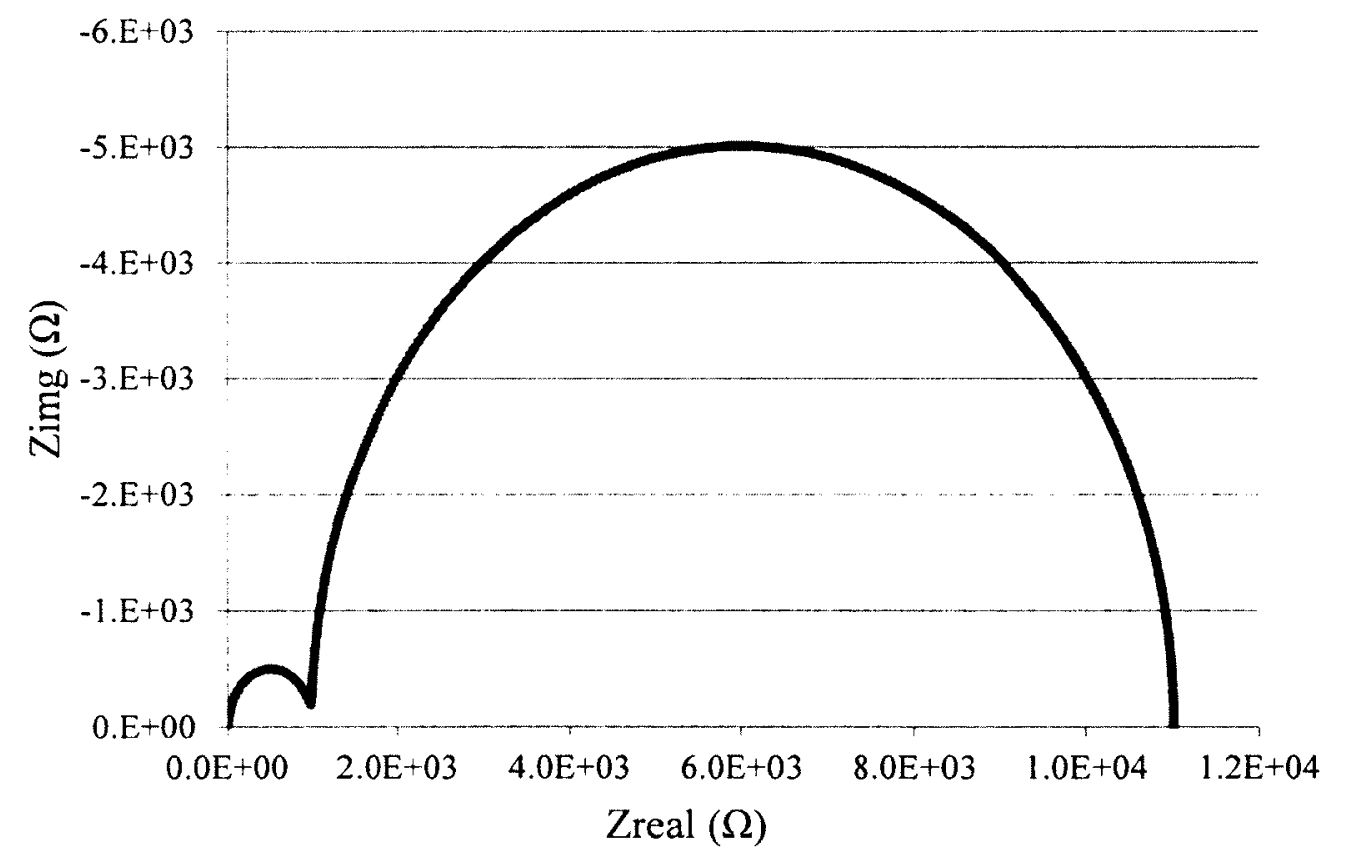

Figure 2-18 Simulated Nyquist plot for a severely damaged coating represented by the circuit shown in Figure 2-14 with $R_{\text {sol }}=10 \Omega, C_{c}=10 \mathrm{nF}, R_{c}=1 \mathrm{k} \Omega$, $C_{d l}=1 \mu F$ and $R_{c t}=10 \mathrm{k} \Omega$. 


\subsection{Summary}

A good coating is one which fully protects the underlying substrate material by preventing the flow of external damaging species. EIS is a technique that measures the impedance of an electrochemical system, and by inference, it can measure how well the coating protects the substrate. A good protective barrier will have a capacitive Bode plot while a breached coating will have resistive traits at the low-frequency end of the EIS spectrum.

Even though equivalent circuit modelling is extensively used to interpret EIS data, the physical meaning of each component in a circuit model is subject to debate. It is crucial that the modeller is able to relate realistic circuit elements to real-world physical and chemical properties in the electrochemical cell.

The simple models described above do not work in every case; section 3.5 contains a literature review where many different circuit models are presented. It is important to always remember that a model which gives an excellent fit to the data is not a good model unless it is a good representation of the real physical system.

Complex equivalent circuits were not used in detail to interpret EIS data in this thesis. Equivalent circuits may provide quantitative information to differentiate between an intact coating and a breached coating but can have difficulties in providing quantitative information on the corrosion occurring in the specimens. The aim of this study is to observe EIS data for protected and corroding samples and correlate the observations with processes occurring in the experimental corrosion cells. 


\section{Chapter 3. Literature Review}

\subsection{Introduction}

This chapter provides a description of the chemistry, general corrosion and pitting corrosion of aluminum, and a review of conventional and new testing methods utilized to assess the efficacy of protective coatings on metals.

\subsection{Corrosion}

Corrosion can be defined as the chemical or electrochemical reaction of a metal with its environment leading to its deterioration [14]. When two dissimilar electrical conductors (electrodes) are immersed in an electrolyte, a galvanic cell is formed, in which chemical energy is converted into electrical energy. Within the electrolyte, current is carried by negative and positive carriers, called ions. The electrode at which positive current enters and chemical reduction occurs is known as the cathode. The electrode at which positive electricity leaves and chemical oxidation occurs is known as the anode. The anode is where the corrosion of metals occurs [14].

Pitting corrosion is a localized form of corrosion by which cavities or "holes" are produced in the material. Pitting is considered to be more dangerous than uniform corrosion damage because it is more difficult to detect, predict and design against. Corrosion products often cover the pits. A small, narrow pit with minimal overall metal loss can lead to the failure of an entire engineering system [14].

Pitting corrosion is initiated by many factors, of which the main ones will be

discussed. Metals such as aluminum, even when uncoated, have a protective oxide film on the surface (discussed later in section 3.3.1). Localized chemical or mechanical 
damage to the protective oxide film can lead to pitting corrosion of the underlying metal. The poor application of a coating or localized damage to a protective coating can leave the underlying substrate susceptible to localized attack and localized corrosion. Also, the presence of non-uniformities in the metal structure of the component, e.g. metallic or non-metallic inclusions can lead to pitting corrosion [14].

When the bare surface of a metal becomes exposed to an aggressive aqueous solution, positively charged metal ions tend to pass from the metal into the solution, leaving electrons behind on the metal $[15,16]$.

$$
M \rightarrow M^{n+}+n e^{-}
$$

The accumulation of negative charge on the metal due to the residual electrons leads to an increase in the potential difference between the metal and the solution. This potential difference is called the electrode potential, or simply, the potential of the metal, which in this case has become more negative. In acid solutions, electrons can react with hydrogen ions, adsorbed on the metal surface from the solution, to produce hydrogen gas [16].

$$
2 H^{+}+2 e^{-} \rightarrow H_{2}
$$

In neutral and alkaline solutions, the reaction to produce hydrogen gas is as follows:

$$
2 \mathrm{H}_{2} \mathrm{O}+2 e^{-} \rightarrow \mathrm{H}_{2}+2 \mathrm{OH}^{-}
$$

The occurrence of the above reactions permits the continued passage of an equivalent quantity of metal ions into solution, leading to corrosion of the metal. On the other hand, the hydrogen ions' concentration can be too low to allow the previous reaction to occur at a significant rate, but electrons in the metal can preferentially react 
with oxygen molecules, adsorbed on the metal surface from air dissolved in the solution, to produce hydroxyl ions [16]. In neutral or alkaline solutions the reaction is as follows:

$$
\mathrm{O}_{2}+2 \mathrm{H}_{2} \mathrm{O}+4 e^{-} \rightarrow 4 \mathrm{OH}^{-}
$$

While in acidic solutions:

$$
\mathrm{O}_{2}+4 \mathrm{H}^{+}+4 e^{-} \rightarrow 2 \mathrm{H}_{2} \mathrm{O}
$$

The process of oxidation involves a loss of electrons by the reacting species in accompanying anodic reactions. The process of reduction involves a gain in electrons in accompanying cathodic reactions. The metal dissolution reaction is the anodic reaction while the reduction of hydrogen ions and oxygen are the cathodic reactions [16]. Figure 3-1 illustrates pitting corrosion on an aluminum sample; this was observed in the coated aluminum samples discussed in Chapter 6 .

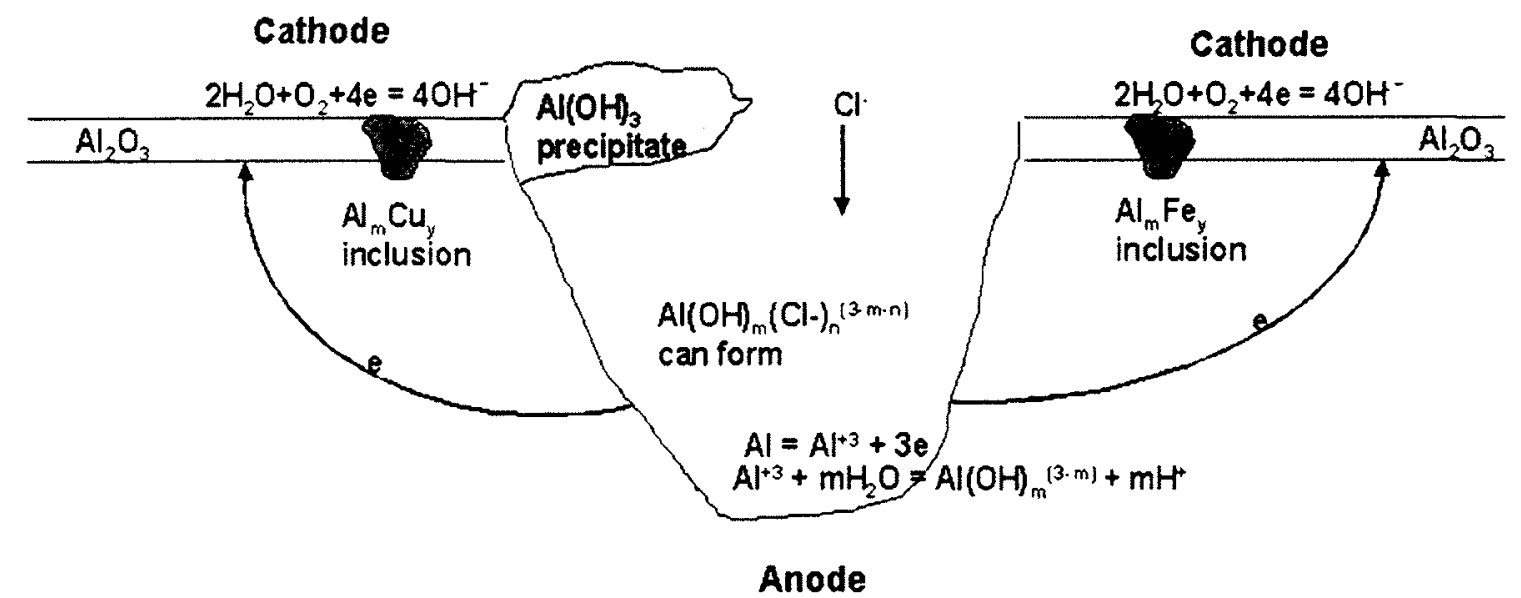

Figure 3-1 Schematic representation of pitting corrosion of an aluminum sample [17].

During pit propagation the $\mathrm{pH}$ inside the pit decreases and becomes more acidic, and when a metal is in a salt water environment, the chloride ions will migrate to the pit to 
balance the positive charge. Effectively a solution of hydrogen chloride is formed which further corrodes the metal and accelerates pit propagation [18].

\subsection{Chemistry and Corrosion of Aluminum}

The following sections discuss the chemistry of aluminum, the environments corrosive to aluminum metal and the mechanisms by which aluminum corrodes.

\subsubsection{Chemistry of Aluminum (Aluminum Oxide)}

Aluminum is an active metal and its resistance to corrosion depends on a layer of oxide, a few nanometers thick, that forms on the surface. The conditions under which such a film is developed can be inferred from a Pourbaix diagram, which is a thermodynamic equilibrium diagram. To use a Pourbaix diagram, the $\mathrm{pH}$ of the solution is assumed to be known and constant in the bulk and at the surface. Pourbaix diagrams are not significantly affected by changes in pressures but are relatively sensitive to temperature changes [15]. Figure 3-2 demonstrates the Pourbaix diagram for Aluminum at $25^{\circ} \mathrm{C}[16]$. 


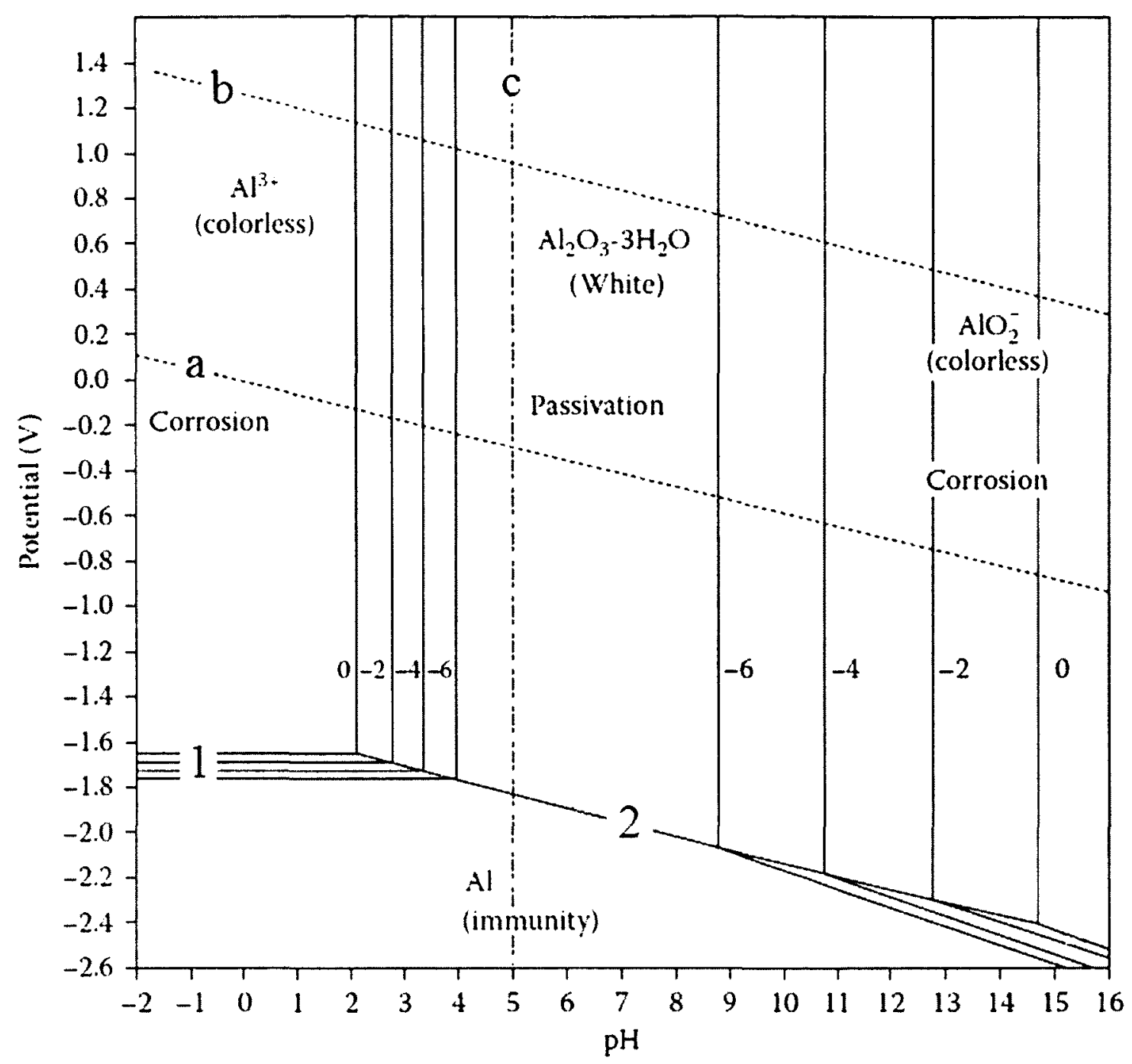

Figure 3-2 Pourbaix diagram for aluminum in water at $25^{\circ} \mathrm{C}$ [15].

Lines ' $a$ ' and ' $b$ ' represent the stability lines for water as a function of $\mathrm{pH}$ and potential; they are present in all Pourbaix diagrams. For potentials above line ' $b$ ', oxygen is evolved at the surface of an immersed electrode according to the half reaction:

$$
\mathrm{H}_{2} \mathrm{O} \rightarrow \frac{1}{2} \mathrm{O}_{2}+2 \mathrm{H}^{+}+2 e^{-}
$$

Below line ' $a$ ', hydrogen gas is evolved from the surface of an immersed electrode according to the half reaction: 


$$
2 \mathrm{H}^{+}+2 e^{-} \rightarrow \mathrm{H}_{2}
$$

Between lines ' $a$ ' and ' $b$ ' water is stable [15].

The vertical and horizontal lines ( 1 and 2$)$ in the Pourbaix diagram represent $\log \left(\right.$ activity $\left.^{1}\right)$ of the soluble species that exist at the boundaries between soluble and solid species. Variation of the activity/concentration leads to several parallel lines. For most applications, when the logarithm (base 10) of the activity is less than -6 , the metal can be considered immune to corrosion [19].

As seen in the Pourbaix diagram, there are four different general regions: an immunity region, a passivation region and two corrosion regions. In the immunity region bounded above by lines 1 and 2, a metal is defined to be immune from corrosion attack and generally safe to use. Line 1 represents the following equilibrium reaction:

$$
3 e^{-}+A l^{3+} \leftrightarrow A l
$$

And line 2 represents:

$$
2 \mathrm{Al}+4 \mathrm{H}_{2} \mathrm{O} \leftrightarrow \mathrm{Al}_{2} \mathrm{O}_{3} \mathrm{H}_{2} \mathrm{O}+6 e^{-}+6 \mathrm{H}^{+}
$$

Corrosion of metals can be reduced with cathodic protection which brings the potential of a metal into the immunity region by forcing a cathodic (negative) shift [16].

In the passivation region, above line 2 and between the vertical lines for soluble species in acid and alkali, a metal tends to become coated with an insoluble oxide or hydroxide that may form on the metal surface. The form of aluminum oxide produced depends on the corrosion conditions; at lower temperatures, aluminum trihydroxide $\mathrm{Al}(\mathrm{OH})_{3}$ is produced [2]. This layer may either be a porous deposit which only partially prevents contact between the metal and the environment, or may be a compact and

\footnotetext{
${ }^{1}$ Activity can be defined as the effective concentration, and is given by: $a=\gamma c$ where $a$ is the activity, $\gamma$ is the activity coefficient and $c$ is the concentration. For dilute solutions, $\gamma$ approaches 1 [14].
} 
adherent film, effectively preventing all direct contact between the metal and the environment [16].

In the third region, corrosion region, thermodynamic calculations indicate that a metal is most stable as an ion in solution and the solid metal is therefore susceptible to corrosion attack [14-16]. At low $\mathrm{pH}$ values, soluble $\mathrm{Al}^{3+}$ ions are formed while at high $\mathrm{pH}$ values soluble $\mathrm{AlO}_{2}{ }^{-}$ions are formed according to the following equations [15]:

$$
\begin{gathered}
\mathrm{Al}_{2} \mathrm{O}_{3} \mathrm{H}_{2} \mathrm{O}+6 \mathrm{H}^{+} \leftrightarrow 2 \mathrm{Al}^{3+}+4 \mathrm{H}_{2} \mathrm{O} \\
2 \mathrm{AlO}_{2}^{-}+2 \mathrm{H}_{2} \mathrm{O} \leftrightarrow \mathrm{Al}_{2} \mathrm{O}_{3} \mathrm{H}_{2} \mathrm{O}+2 \mathrm{OH}^{-}
\end{gathered}
$$

It can be said, for example, that a stable oxide film only occurs when aluminum is in the $\mathrm{pH}$ range of $4 \sim 9$. The point where soluble species are in equilibrium with each other, but at extremely low activity, corresponds to a $\mathrm{pH}$ of approximately 5 (line ' $\mathrm{c}$ '), where the oxide is most stable, and is represented by the following equation [15]:

$$
\mathrm{Al}^{3+}+2 \mathrm{H}_{2} \mathrm{O} \leftrightarrow \mathrm{AlO}_{2}^{-}+4 \mathrm{H}^{+}
$$

If an aluminum sample is in the passive region as shown in the Pourbaix diagram, it does not corrode uniformly but it can still be susceptible to pitting corrosion. In aerated solutions, oxygen reduction is the cathodic reaction, and halide ions, such as chloride ions, accelerate the corrosive anodic reactions. Corrosion will occur in the passive region if the aluminum sample is polarized to its pitting potential [15]. To further understand this phenomenon, one must first understand the polarization/Evans diagram. Pourbaix diagrams only present the thermodynamic conditions for the metal samples, but attention must be paid to the kinetics of corrosion.

The polarization curve is typically presented with potential (V) on the verticalaxis, and current density $\left(\mathrm{A} / \mathrm{mm}^{2}\right)$ on the horizontal-axis of the plot, usually on a 
logarithmic scale [14-16]. The potential-current density relationship of the metal under test can be determined by two main methods, galvanostatic (current control) and potentiodynamic (voltage control) polarization.

In the galvanostatic method, a fixed current is applied from a constant-current power source connected between the working electrode and a counter electrode, and the change in potential induced in the working electrode by the passage of current is measured against the reference electrode [16].

In the potentiodynamic method, the potential of the working electrode is controlled at a fixed value and the current is monitored. When the area of the sample under investigation is taken into account, the 'current density' $\left(\mathrm{A} / \mathrm{mm}^{2}\right)$ is monitored as opposed to current (A). The potentiodynamic method has the advantage that it can follow more closely the behaviour of metals during the formation and breakdown of passivating films, where the behaviour primarily depends on the potential of the metal, and very large changes in currents can occur at constant potential [16].

By the application of current/voltage, the potential of the metal surface is polarized. When the current is applied in a positive direction, it is said to be anodically polarized, and when applied in a negative direction, it is cathodically polarized. The degree of polarization is a measure of how the rates of the anodic and the cathodic reactions are retarded by various environmental and/or surface processes. Environmental factors include concentration of metal ions, dissolved oxygen, $\mathrm{pH}$, etc., in solution; and surface factors may include adsorption, film formation, ease of release of electrons, etc [16]. 
The variation of current as a function of potential (a polarization curve) allows the study of the effect of concentration and activation processes on the rate at which anodic or cathodic reactions can give up or accept electrons. Polarization measurements can thereby determine the rate of the reactions that are involved in the corrosion process - the corrosion rate [14-16].

Figure 3-3 shows anodic and cathodic polarization curves that represent anodic and/or cathodic reactions. The relative values of the "slopes" of the anodic or cathodic polarization curves determine whether anodic, cathodic, or both reactions control the rate of the corrosion process. These polarization/Evans diagrams are used extensively by corrosion scientists and engineers to evaluate the effect of various factors on corrosion rates [14-16].

If the anode and cathode are on the same metal surface, then a current cannot be measured unless the sites are well separated. The corrosion potential can be measured against a convenient reference electrode [15]. 


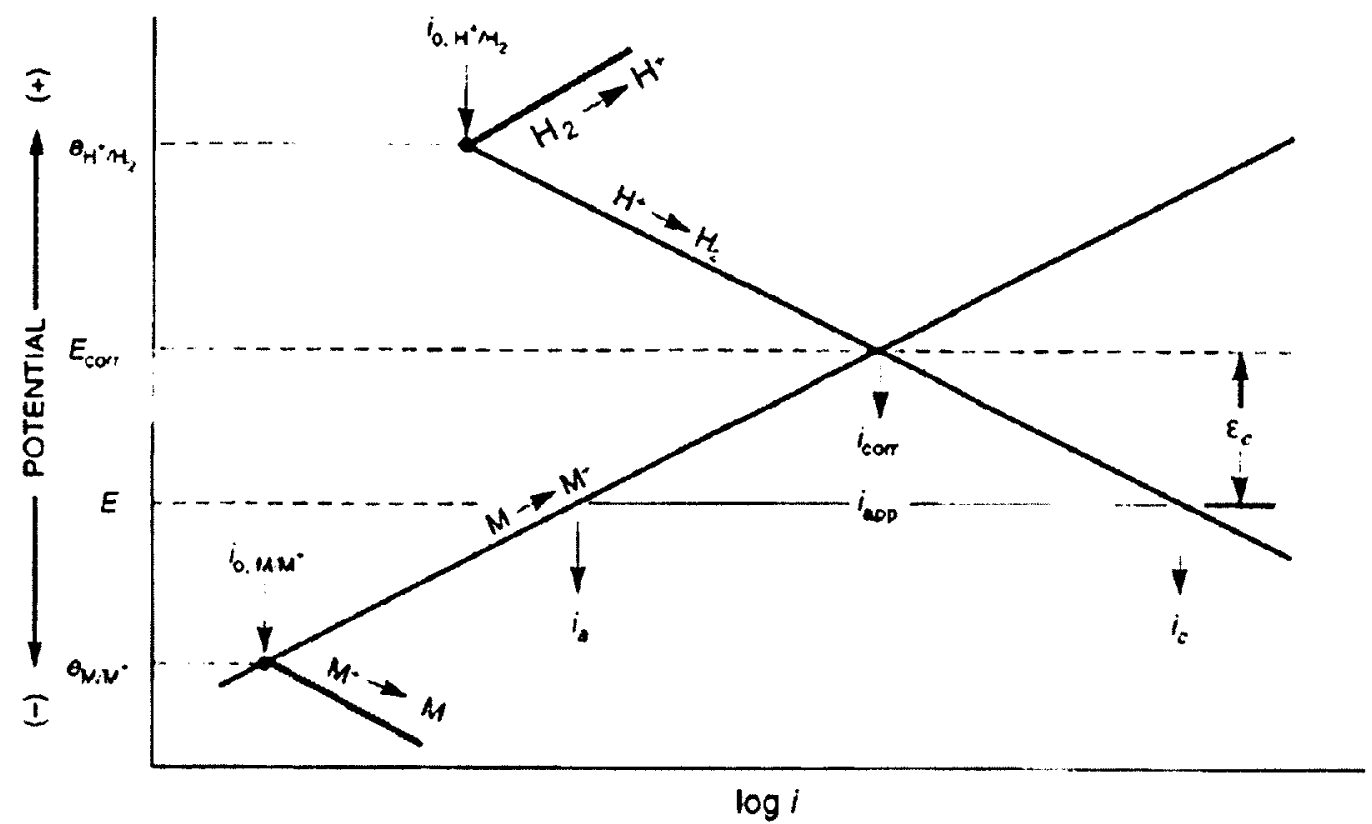

(a)

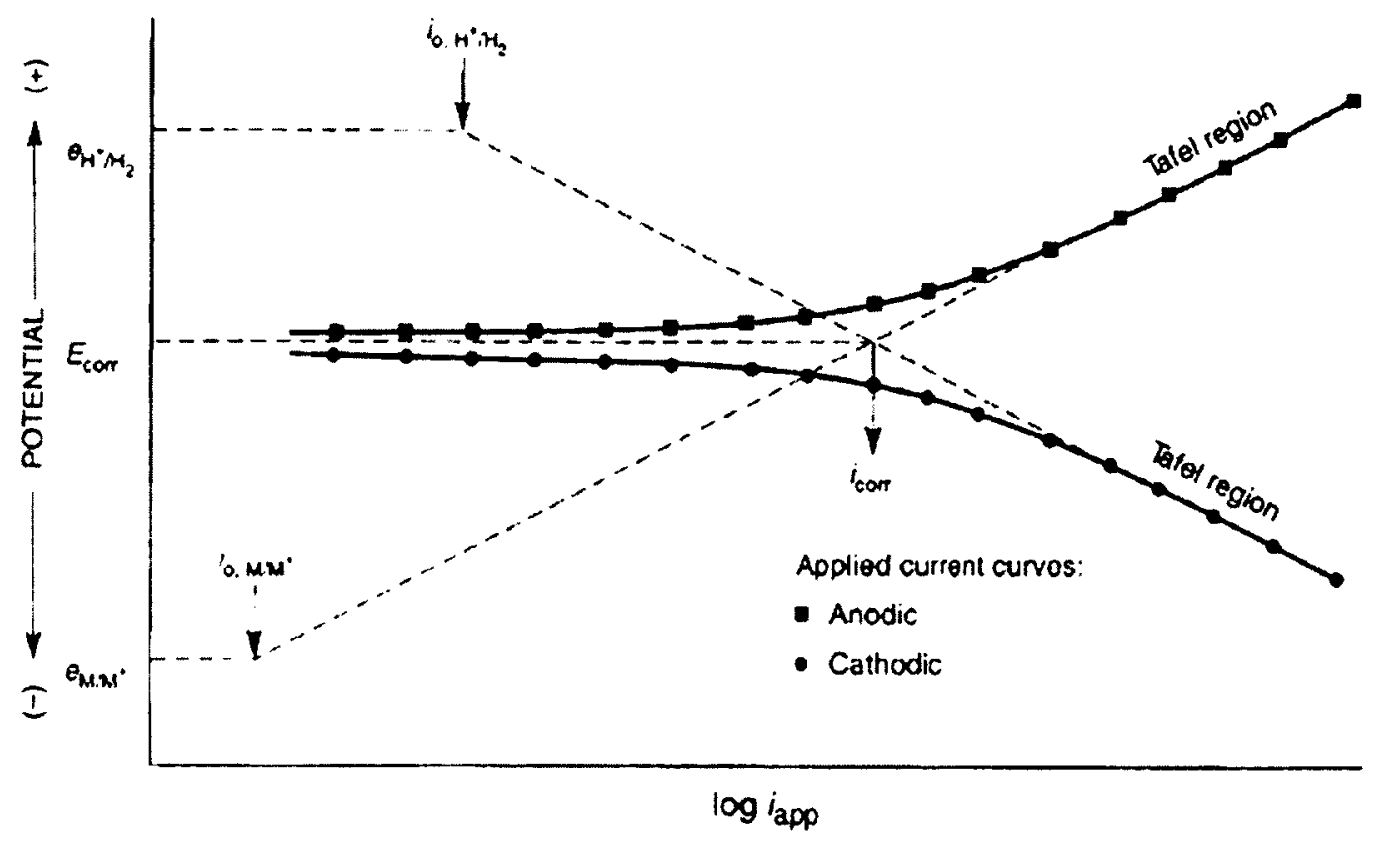

(b)

Figure 3-3 Schematic representation of typical polarization curves: (a) cathodic and anodic half-cell reactions demonstrating half-cell potentials $e_{\mathrm{H}+/ \mathrm{H} 2}$ and

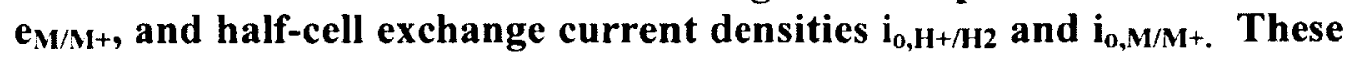
give a corrosion potential $E_{\text {corr }}$ and corrosion current $i_{\text {corr }}$ with an overpotential of $\varepsilon_{c}$ compared to the mixed-potential/equilibrium (corrosion) potential; (b) simulated experimental polarization curves derived from (a) [19]. 
The cathodic and anodic currents are equal at the corrosion potential, $\mathrm{E}_{\text {corr, }}$ where the current is equal to the corrosion current, $\mathrm{i}_{\text {corr }}$. If the potential is shifted positively with respect to this equilibrium value, the anodic reaction will accelerate, in the absence of passivation. If the shift from this equilibrium is negative, the cathodic reaction will accelerate. Figure $3-3 \mathrm{a}$ shows the application of a cathodic current, $\mathrm{i}_{\text {app }}$, with an overvoltage (negative) of $\varepsilon_{c}$.

The overvoltage of a half-cell reaction is the difference between the half-cell voltage $\left(\mathrm{e}_{\mathrm{H}+/ \mathrm{H} 2}\right.$ or $\left.\mathrm{e}_{\mathrm{M} / \mathrm{M}+}\right)$ and the applied voltage $(\mathrm{E})$.

The overpotential or overvoltage of the mixed-potential/equilibrium (corrosion potential), is the difference between the corrosion potential $\left(E_{c o r r}\right)$ and the applied potential $(E)[19]$.

$$
E_{\text {corr }}-E=\varepsilon_{c}
$$

The measured current density, $i$, is proportional to the reaction rate and is composed of an anodic current density $i_{a}$ and a cathodic current density $i_{c}$. When $i_{a}>\left|i_{c}\right|, i$ is positive and there is a positive overpotential, and vice versa [20].

A corroding metal in a solution takes up a potential, the corrosion potential, such that the rate of the anodic reaction is equal to the rate of the cathodic reaction. If the potential of the corroding metal is displaced slightly from the corrosion potential, $\mathrm{E}_{\mathrm{cor}}$, either by galvanostatic or potentiodynamic polarization, then the potential $\mathrm{E}$ is initially a linear function of the current density $i$, the current per unit area. The potential-current density plot is approximately linear in a region of within $\pm 10 \mathrm{mV}$ of the corrosion potential. The slope of the linear polarization curve $\mathrm{dE} / \mathrm{di}$ is termed the polarization 
resistance $R_{p}$. The polarization resistance is inversely proportional to the rate of corrosion expressed as the equivalent corrosion current density $i_{\text {corr, }}$ given by [16]:

$$
i_{\text {corr }}=\frac{B}{R_{p}}
$$

where $B$ is a constant.

As the displacement of the potential of the metal from the corrosion potential is increased, the polarization curve increasingly deviates from the linear relationship between potential and current density until a region is reached when the potential exhibits a linear dependence on the logarithm of the current density. This is the Tafel region of Figure $3-3 \mathrm{~b}$. In the region of the corrosion potential, the relationship between the observed potential and current density $i$ is given by [16]:

$$
\eta=b \log \frac{i}{i_{o}}
$$

There are two such relationships: one with a positive slope for the anodic reaction and one with a negative slope for the cathodic reaction. These two lines cross at the corrosion potential where $\varepsilon_{\mathrm{c}}$ is zero. The overvoltage is $\eta$. The term $i_{o}$ is the exchange current density, a measure of the electron exchange rate in the half-cell reaction at the open-circuit anode or open-circuit cathode (redox reactions) potentials. It is the current at the point where the Tafel line intercepts the half-cell voltage (see Figure 3-3b). The constant $b(\mathrm{~V})$, named the Tafel slope, is the slope of the polarization curve; this Tafel slope is related to the kinetics of the corrosion reaction. For the cathodic reactions of hydrogen-ion reduction or oxygen reduction, the value of $b$ is generally about $-120 \mathrm{mV} /$ decade and for metal dissolution reactions from active surfaces (anodic reactions), values of $b$ are generally in the range of $30 \mathrm{mV}$ to $70 \mathrm{mV}$ [16]. 
Metals and alloys that exhibit passive behaviour display a very distinct evolution on the Evans diagram, as shown in Figure 3-4.

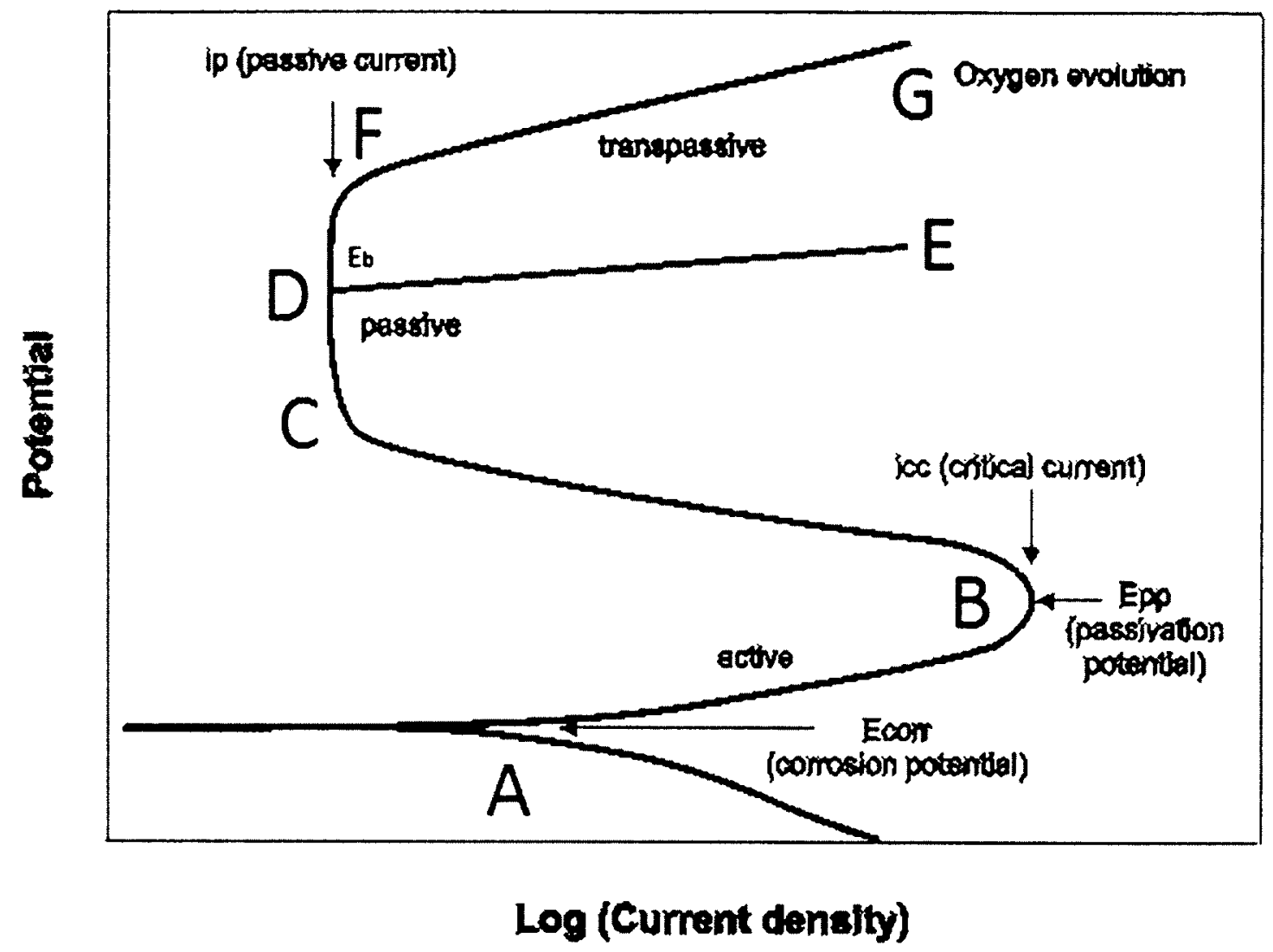

Figure 3-4 Evans diagram for active-passive metals like aluminum [21].

As the potential is increased from the corrosion potential (point A), the current density increases due to an increase in the rate of metal dissolution. The highest rate of corrosion is achieved at a maximum current density known as the critical passivation current density $i_{c c}$ (point $B$ ). The potential corresponding to $i_{c c}$ is called the primary passivation potential $E_{p p}$. This voltage represents the transition of the metal from an active state to a passive state, and defines the beginning of the formation of an oxide film on the metal surface [14-16]. This passivation potential depends on the $\mathrm{pH}$ value of the solution and can also depend on the presence of anions, such as $\mathrm{Cl}^{\circ}$, which may favour or 
hinder passivation; the nature of the anions in solution plays an important role in the stability of the oxide film (see Figure 3-2) [14-16]. The value of the critical passivation current density gives a measure of the ease of passivation; the smaller the current density, the easier is passivation $[15,16]$.

As a result of the formation of an adherent oxide film causing passivity of the metal, the current density starts to decrease, by orders of magnitude, until it reaches the Flade potential (Point C). At this potential, the current density becomes virtually independent of potential and remains virtually stationary and is called the passive current density $i_{p}$. The Flade potential is generally close to the critical passivation potential and depends on the composition of the solution. The passive current density which passes through the passivating film is a measure of the protectiveness of the film. At $i_{p}$, the metal dissolution occurs at a constant rate and the dissolved film is immediately replaced by a new film; a net balance is maintained between the dissolution and film reformation [15, 16].

As the potential of a metal in the passive state is increased, the passive current density does not change (curve $C D$ ). Eventually, the current density begins to rapidly increase with potential, point $D$. Such increase in current density may be attributed to localised breakdown of the oxide film by anions, particularly chloride ions. This oxide breakdown usually occurs at weak points, generally associated with a surface defect such as a discontinuity in the grain boundary, a dislocation or an inclusion in the metal/oxide, leading to exposure of the underlying metal. When the bare metal is exposed to the aggressive anions, it can dissolve, giving rise to an increase in the anodic current. Continued dissolution can lead to the formation of pits $[15,16]$. 
The breakdown of the oxide by anions with the initiation of pitting occurs at a critical potential called the critical breakdown or pitting potential $\mathrm{E}_{\mathrm{b}}$, and the current density then increases rapidly with potential, curve $D E$. The value of $E_{b}$ depends on the ratio of inhibitive anions (which stabilise the passivating oxide film) and aggressive anions (which break down the oxide film). If the ratio of inhibitive to aggressive anions is sufficiently high, the breakdown of the film by the anions may be completely suppressed and no critical breakdown potential is observed. As the ratio of inhibitive to aggressive anions decreases, the breakdown potential is observed to become more negative $[15,16]$.

Sometimes, depending on the composition of the solution, breakdown of the film by aggressive anions does not occur in the passive region but rather at a higher potential, along FG, and oxidation of the metal occurs to produce a soluble ion. This process is known as transpassive dissolution $[15,16]$. Alternatively, curve FG may also be associated with increased current as water is oxidized and oxygen production occurs.

One of the strategies employed in the protection of metals is the anodization process. An anodic potential is applied so that the sample is in the passive state (between B and C), and by choosing the right media and applied current, it is possible to grow very thick oxide layers to protect the metal surface from oxidation. This constitutes anodizing, a well used technique in the protection of aluminum alloys [14-16].

\subsubsection{Corrosive Environments}

The presence of oxygen greatly affects the corrosion of aluminum. In deaerated solutions, the corrosion is slower, while with the presence of oxygen, it is accelerated. In acid solutions; the higher the concentration of oxygen, the greater is the corrosion of the aluminum [15]. Oxygen is an oxidizing agent, and the effect of varying the activity of 
oxidizing agents is shown in Figure 3-5. In this figure, a metal $M$ is immersed in an electrolyte and curves 1 to 7 represent increasing oxidizer activity from 1 to 7 [19].

Initially, at 1 , the metal is in the active region and corrodes at a rate corresponding to point $A$. As the activity of the oxidizer is increased from 1 to 3 , the corresponding corrosion rate increases as well; point $\mathrm{A}$ to $\mathrm{C}$. In this particular range of oxidizer activity, corrosion rate increases with increasing oxidizer activity [19].

By further increasing oxidizer activity, to 4, a rapid transition in corrosion potential from point $\mathrm{D}$ (active state) to point $\mathrm{G}$ (passive state). As oxidizer activity is increased from 4 to 5 , the metal remains in the passive state and its corrosion rate remains low and constant. By increasing the oxidizer activity further, the transpassive region is reached, and corrosion rate rapidly increases; curves 6 and 7 [19].

By reducing oxidizer activity, for example deaerating the electrolytes, the opposite happens. Assuming the system is at an activity of 7 to start with, the metal will be corroding at point $J$. If the activity of the oxidizer is now reduced, corrosion rate will decrease from point $\mathrm{J}$ to point $\mathrm{I}$. Further reductions in the oxidizer activity will result in a system shift into the passive region; points $H, G$ and $F$. By reducing the oxidizer activity down to curve 2, a transition from $\mathrm{E}$ to $\mathrm{B}$ occurs, and as it is further reduced to curve 1 , the corrosion rate will decrease from point $\mathrm{B}$ to point $\mathrm{A}$ [19].

The path during oxidizer additions is $A, B, C, D, G, H, I, J$, and as the oxidizer is reduced, the path followed is J, I, H, G, F, E, B, A [19]. 


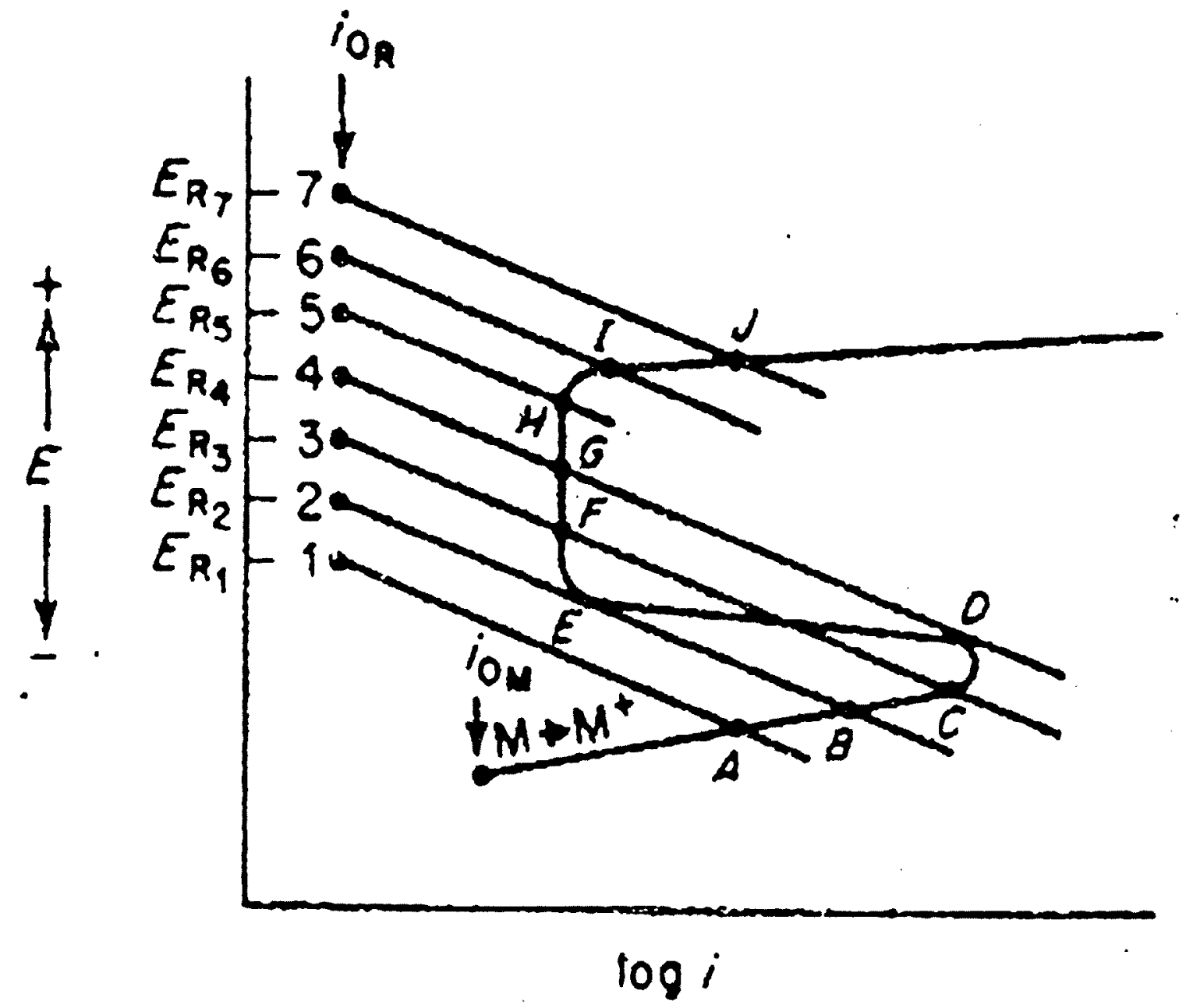

Figure 3-5 Effect of oxidizer concentration on the electrochemical behavior of an active-passive metal [19].

Hydrogen and nitrogen gases have no effect on the corrosion of aluminum, while the presence of carbon dioxide might have a slight inhibiting effect on the corrosion of Aluminum in aqueous solutions [15].

Aluminum corrodes in acidic and alkaline conditions. In acidic conditions, $\mathrm{Al}^{3+}$ ions are produced, while in alkaline conditions, $\mathrm{AlO}_{2}{ }^{-}$ions are produced. A few exceptions exist where the aluminum might not corrode in acidic or alkaline conditions, the oxide layer may not be soluble, or the solution might have an oxidizing nature where 
the oxide film is maintained. In general, there is no specific relation between the $\mathrm{pH}$ and the rate of corrosion of aluminum [15].

Aluminum does not generally uniformly corrode in fresh/distilled water even at elevated temperatures [15]. On the acidic side, phosphoric acid in intermediate concentrations has detrimental effects on aluminum. Even dilute $(<1 \%)$ phosphoric acid solutions result in etching of the aluminum surface. Dilute sulfuric acid solutions, $\sim 10 \%$ in concentration, can lead to significant corrosion of aluminum. Aqueous solutions of hydrofluoric, hydrochloric and hydrobromic acid solutions are also strongly corrosive to aluminum at concentrations higher than $0.1 \%[15]$.

On the higher range of the $\mathrm{pH}$ scale, solutions of sodium hydroxide or potassium hydroxide are very detrimental to aluminum except at extremely low concentrations, $<0.01 \%$. Most inorganic salt solutions at or close to neutral $\mathrm{pH}$ cause negligible uniform corrosion of aluminum at room temperature, but such solutions cause localized corrosion; infamously, pitting corrosion [15]. 


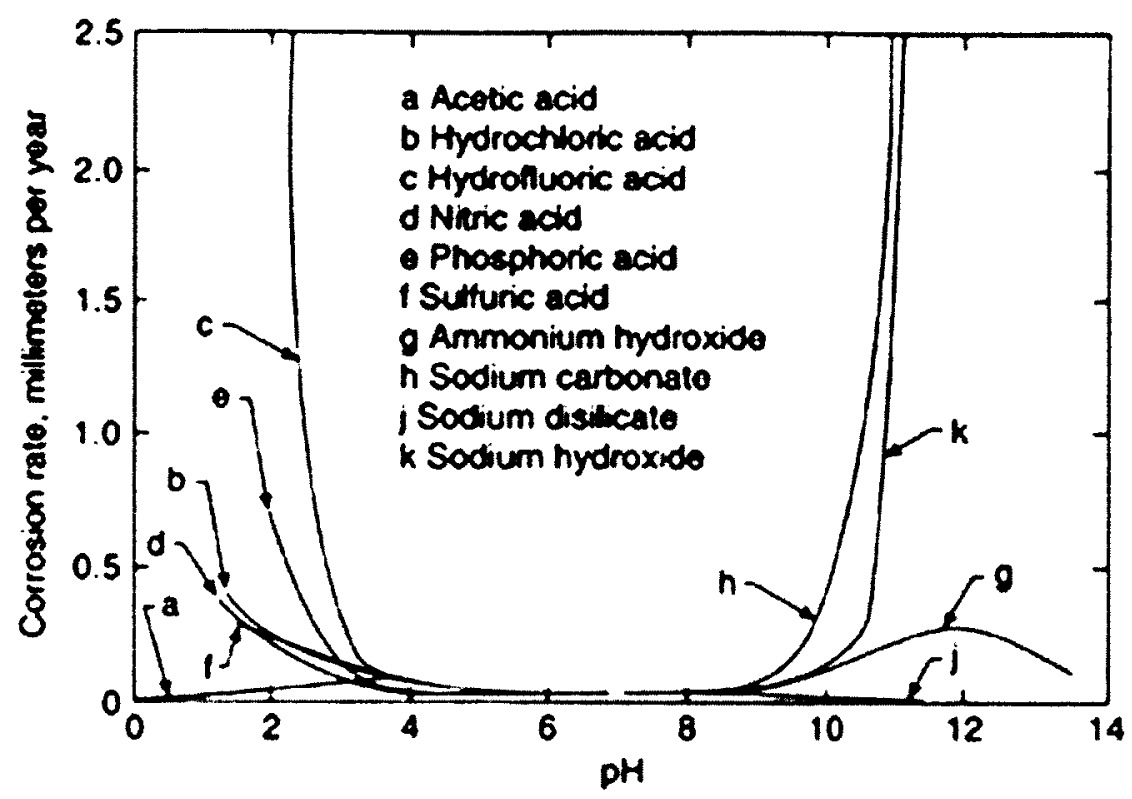

Figure 3-6 Schematic representation showing the relationship between pH of several solutions and the corresponding corrosion rate of aluminum [15].

\subsubsection{Corrosion of Aluminum}

Aluminum and its alloys generally act as the anode in galvanic cells, which means that they may corrode sacrificially to protect other more noble metals to which they are coupled, whether inadvertently (impurities and inclusions in the aluminum itself) or deliberately. As stated earlier in section 3.3.1, the aluminum is protected in the $\mathrm{pH}$ range of $4 \sim 9$ by its oxide, but, aluminum may still corrode due to defects in this oxide film. Resistance to corrosion is greatly improved as the purity of aluminum is increased, but even on the purest aluminum, the oxide film may still contain defects [15].

In pitting corrosion of aluminum, fine, white, gelatinous deposits of aluminum hydroxide often cover deep pits. Pitting corrosion is initiated at weak points of the oxide or hydroxide film [15]. In aerated solutions, the cathodic reaction is oxygen reduction, and the development of such a reaction can be prevented or at least slowed by the 
removal of the oxygen. In the absence of oxygen, the cathodic reactions can be insufficient to polarize aluminum to its pitting potential [15] (see Figure 3-5).

Pitting corrosion of aluminum generally occurs in four stages. Initially it occurs at the boundary of the passive film and the solution. After the penetration of the oxide film, processes occurring within the passive film lead to a formation of so-called metastable (unstable) pits. These metastable pits initiate and grow for a short period of time below the critical pitting potential and then repassivate (an intermediate step in pitting). Above the critical pitting potential, these unstable pits turn into stable pits, and continuous pitting occurs [22], to the detriment of the metal.

Figure 3-7 demonstrates the potentiodynamic scan of a sample, with the arrows showing the start and end points of the curve. Such plots can reveal the formation of pits, observed as spikes on the curve. $E_{b d}$ represents the breakdown potential (also known as the critical pitting potential) at which the surface oxide film breaks down [20]. The metastable pits are very small in size. They grow and repassivate at in a very short amount of time, as little as a few seconds [22]. After the voltage is reduced, on the return path, the current density and corrosion rate stay high, until repassivation starts to occur, and the current density drops, leading to a new higher corrosion potential at $\mathrm{E}_{\mathrm{rp}}$. 


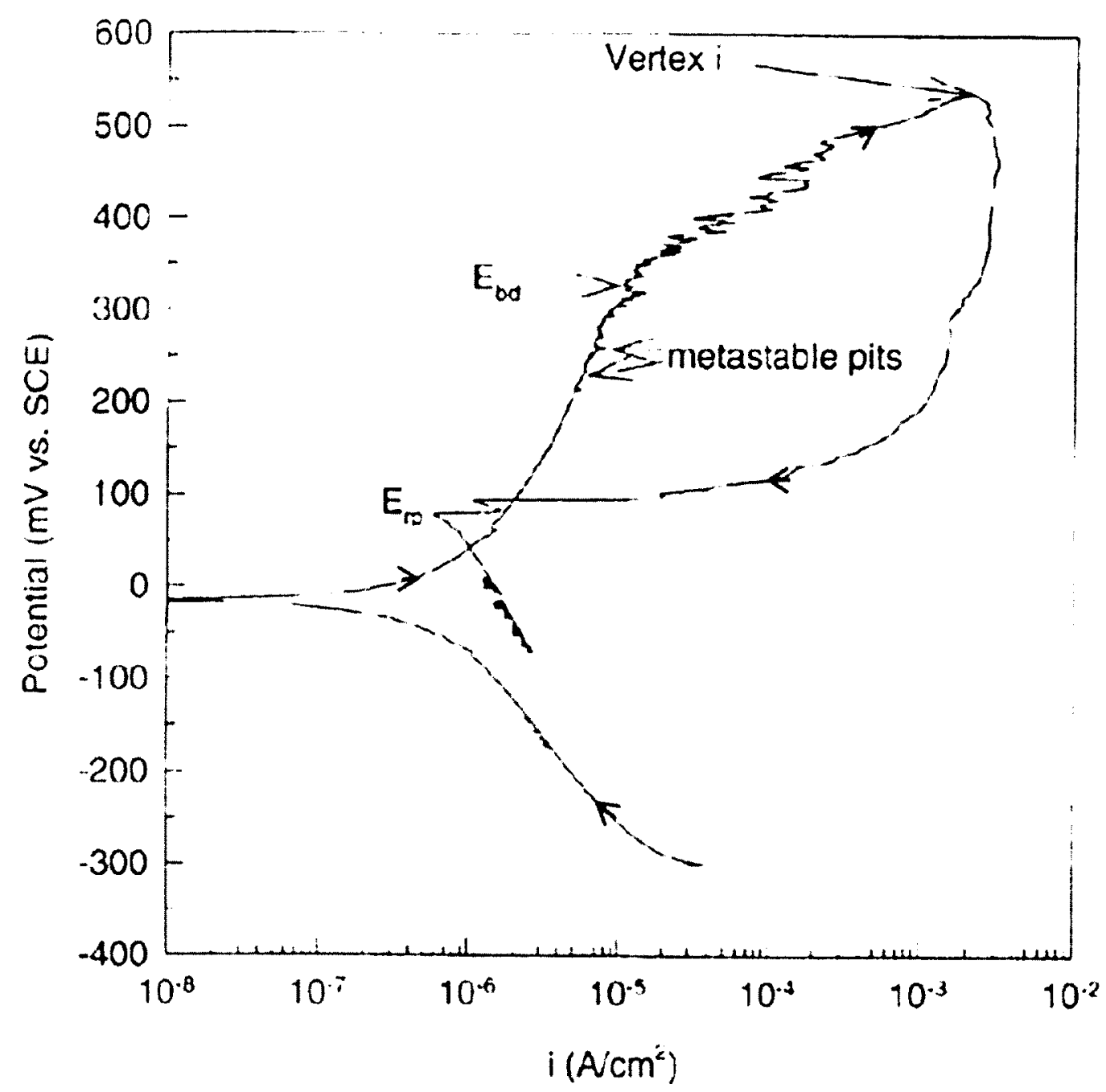

Figure 3-7 Sample polarization curve demonstrating the formation of pits [20].

The current density increases when metastable pits first occur and begin to grow, and then after a short time decreases as the pit repassivates. Metastable pits can form several hundred millivolts below the pitting potential (breakdown potential). Generally, metastable pitting events occur more frequently at potentials close to the pitting potential [22]. For pure aluminum metal, the number of metastable pits can increase for two main reasons: an increase of the anodic potential (up to the pitting potential), and with an increase in the concentration of chloride ions at a constant potential [22]. 
It is generally accepted that a necessary condition for stable pit growth is the presence of a salt film at the pit's bottom [15]. These salt films are a result of high dissolution rate within the pit and the consequent acidification of the solution. There are two types of possible pit salts, aluminum chloride $\left(\mathrm{AlCl}_{3}\right)$, and aluminum oxychlorides $\left(\mathrm{Al}(\mathrm{OH})_{2} \mathrm{Cl}\right.$ and $\left.\mathrm{Al}(\mathrm{OH}) \mathrm{Cl}_{2}\right)$. The chloride salt films are stable only in a highly acidic solution, $\mathrm{pH} \sim 1$ for $\mathrm{AlCl}_{3}$ [15].

The pitting mechanism of aluminum in the presence of chloride ions can be summarized as [15]:

- $\mathrm{Cl}^{-}$adsorption in micro-flaws of the oxide film;

- Slow oxygen reduction at the cathodic sites;

- Dielectric breakdown of the oxide film;

- Fast oxidation of bare aluminum producing soluble chloride and oxychloride complexes at the bottom of the flaws or pits;

- Dissolution of chloride complexes or repassivation of pits;

- Propagation of the micro-pits (formed in the previous step);

- Hydrolysis of soluble chlorides/oxychlorides resulting in acidification of the solution within the pits;

- Hydroxide dissolution inside pits and precipitation of aluminum hydroxide outside pits (in neutral solution) resulting in the formation of tree-like accumulations of corrosion products at the mouths of the pits;

- Aluminum corrosion inside the pits due to the aggressive hydrochloric acid solutions; 
- Possible repassivation and pit death (the chloride/oxychloride film is dissolved and replaced by a passive oxide film).

Some of the micro-pits repassivate, while some propagate to larger pits [14-16]. During the process of aluminum corrosion, some of the anodic reactions are $[15,16]$ :

$$
\begin{gathered}
A l \rightarrow \mathrm{Al}^{3+}+3 e^{-} \\
\mathrm{Al}^{3+}+3 \mathrm{H}_{2} \mathrm{O} \rightarrow \mathrm{Al}(\mathrm{OH})_{3}+3 \mathrm{H}^{+}
\end{gathered}
$$

In neutral solution, the consumption of hydroxide ions at anodic sites can make the $\mathrm{pH}$ more acidic. This can be accompanied by the migration of chloride ions, which facilitate the anodic reactions, forming soluble aluminum chlorides, which in turn give hydroxides and acids by hydrolysis.

$$
\mathrm{AlCl}_{3}+3 \mathrm{H}_{2} \mathrm{O} \rightarrow \mathrm{Al}(\mathrm{OH})_{3}+3 \mathrm{HCl}
$$

Cathodic sites are frequently more alkaline because of the consumption of acid and formation of hydroxides locally. The reactions taking place at the cathode depend on the $\mathrm{pH}$ and oxygen concentration and are $[15,16]$ :

$$
\begin{gathered}
3 \mathrm{H}^{+}+3 e^{-} \rightarrow \frac{3}{2} \mathrm{H}_{2} \\
\frac{3}{4} \mathrm{O}_{2}+\frac{3}{2} \mathrm{H}_{2} \mathrm{O}+3 e^{-} \rightarrow 3 \mathrm{OH}^{-} \\
3 \mathrm{H}_{2} \mathrm{O}+3 e^{-} \rightarrow \frac{3}{2} \mathrm{H}_{2}+3 \mathrm{OH}^{-}
\end{gathered}
$$

\subsection{Conventional Testing Methods}

Several tests and standards have been created for the corrosion testing of metals. Cabinet tests are one of the oldest techniques, used since the 1900's for the evaluation of coatings against corrosion. They were also used as means for performing accelerated corrosion 
testing. As the name suggests, these tests are performed in a chamber, or cabinet, where a corrosive environment is simulated. Some common corrosive environments produced in a test chamber are salt fog, humidity, hot and cold temperatures, ultraviolet exposure, and corrosive gases. These environments may be used individually or in combination with each other. With technology, these tests have evolved from home-made cabinets that operated at fixed temperatures and humidity, to complex computer controlled tests with varying service conditions. There have also been advancements in the size of the cabinets to accommodate various samples. They have evolved from bench-top cabinets to walk-in and even drive-in chambers $[23,24]$.

Salt-Spray (FOG) Tests are the most common type of cabinet tests. In this procedure, the samples are exposed to a fog which is produced by air atomizing of sodium chloride solution, while controlling the cabinet temperature. Salt-spray tests have a widespread use in many industries including but not limited to automotive, aerospace, paints and coatings. These tests can be performed as standalone tests or in conjunction with other tests. The duration of these tests can be up to 5000 hours, during which the chamber has to be closed for the duration, excepting occasions when samples need to be rotated or visual inspected.

The results of the FOG tests are usually interpreted based on visual inspection of the failure of the coatings, or based on the total mass loss of the sample. Not infrequently, such tests have been misapplied and misused. Sometimes there is no clear correlation between salt-spray tests and failures in the natural environment. Strictly, these tests cannot be used as quantitative indicators of corrosion, but can be useful in controlling the quality of a coating process. After a sample is prepared, it is exposed in a FOG chamber, 
and if the sample does not survive a certain number of hours in the chamber, it can provide an indication that the coating process of the sample might not have been done properly $[23,24]$. Unfortunately, the correlation between survival durations in salt spray tests and the life expectancy of coatings is often weak $[25,26]$.

The standard FOG tests have other variations designed for different materials. Certain aluminum alloys, when exposed to marine atmospheres, develop surface blisters followed by metal delamination: exfoliation. Cyclic acidified salt-fog tests are used for exfoliation testing. In these experiments, $5 \%$ sodium chloride solutions, adjusted to $\mathrm{pH}$ values between 2.8 and 3.0 with the use of acetic acid, are utilized. Another variation of this test is the acidified synthetic-seawater (FOG) test. Instead of $5 \%$ sodium chloride solution, a synthetic seawater solution with $\mathrm{pH}$ adjusted to values between 2.8 and 3 is used. Many other variations of the FOG tests exist, often applicable to materials other than aluminum [23].

Humidity tests are categorized into either controlled humidity tests which target a specific relative humidity, or high humidity tests which are conducted at high humidity ranges, typically in excess of $95 \%$. In the humidity range of $70 \%$ to $95 \%$, filiform corrosion, identified by thread like strands, is formed under finishes such as powderclear-coated aluminum. Testing for filiform corrosion is initiated with salt spray exposure for $6 \mathrm{~h}$ followed by exposure to $70-90 \%$ humidity. Visual observations are used to evaluate all the aforementioned tests [23].

As mentioned earlier in section 3.3.3, polarization methods can be used to assess aluminum corrosion. The Ford anodized aluminum corrosion test (FACT) involves the cathodic polarization of the anodized aluminum surface with the use of a small, 
cylindrical, glass clamp-on cell. A $5 \% \mathrm{NaC} 1$ solution containing cupric chloride $\left(\mathrm{CuCl}_{2}\right)$ and acidified with acetic acid is used as the electrolyte, and a voltage is applied across the cell by using a platinum auxiliary electrode. The cathodic polarization creates alkaline conditions which promote dissolution at small defects in the anodized aluminum. The cell voltage is monitored for $3 \mathrm{~min}$ and the cell voltage over time is recorded. After extensive testing, it has been found that some aluminum alloys failed the test but still performed well in service and hence this standard has been withdrawn [23].

The cathodic breakdown test, a similar test, involves cathodic polarization to $-1.6 \mathrm{~V}$ versus saturated calomel electrode (SCE) for a period of $3 \mathrm{~min}$, in acidified $\mathrm{NaCl}$ solution, and the cell voltage over time is recorded. This test was also designed for anodized aluminum alloys. Similarly, the alkali created at the large applied currents will promote the formation of corrosion spots at defects in the anodized film as in the previous test [23].

Another test method for anodized aluminum is the Single Frequency Impedance Test. This method uses a $1 \mathrm{~V}$ and $\mathrm{l} \mathrm{kHz}$ signal to determine the impedance of sealed (post-anodizing treatment) anodized aluminum. The sample is immersed in $3.5 \% \mathrm{NaCl}$ [23].

Electrochemical noise (EN) methods have also been explored as a method to analyze the degradation of polymer-coated metals [23], and this has been in practice since the 1970's [25]. The spontaneous potential and current fluctuations (noise) that occur in electrochemical systems are monitored as a function of time [27]. This technique does not pose artificial disturbance to the system, data collection is simple and the instrumentation is of low cost, which gives it a great advantage. Besides the direct analysis of the shape, 
the size and the distribution of the noise current and noise potential transients in the time domain, three different main approaches have been proposed for the data analysis of the electrochemical noise technique: statistical, spectral and chaos-theory-based methods [25]. These methods will not be explored as they are beyond the scope of this thesis. Although, in principle, information about both the rate and the mechanism of the corrosion process can be extracted [27], this is not easy. The main difficulty which has driven many researchers away from EN method is the considerable difficulty faced in data interpretation [25].

\subsection{Conventional EIS Studies of Coatings}

EIS can provide information concerning water uptake in coatings, changes in the resistive properties of the polymer layer(s), development of coating delamination, and initiation and propagation of corrosion at the metal/coating interface [25].

The most popular method to analyze EIS data is by fitting the spectra to an appropriate equivalent circuit (EC) [28-34]. The components of the circuit and their experimental values can be related to physical features.

It has been established, in many papers [26,35-41], that in EIS studies of coatings, data showing capacitive behaviour and high impedances $\left(>10^{8} \Omega \mathrm{cm}^{2}\right)$ at low frequencies $(<1 \mathrm{~Hz})$, demonstrate good protective coatings. These high impedances can sometimes be difficult to measure. It has also been concluded that poor or damaged coatings display resistive traits with lower impedance values $\left(<10^{6} \Omega \mathrm{cm}^{2}\right)$ in the lower frequency range $(<1 \mathrm{~Hz})$.

Four general models, Figure 3-8, have been created and have been generally used by researchers to model EIS data [9,29-34]. 
(a)

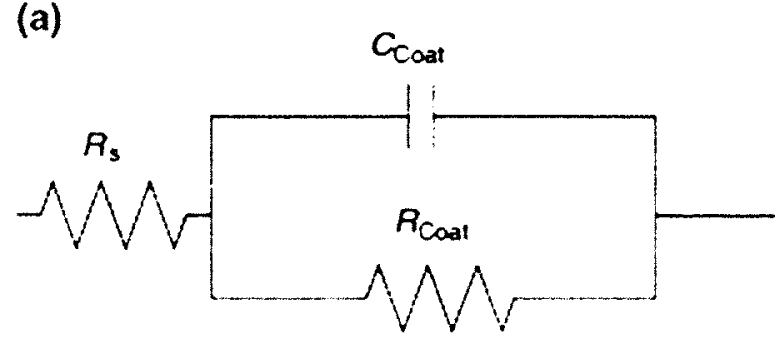

(b) $\quad c_{\text {coal }}$

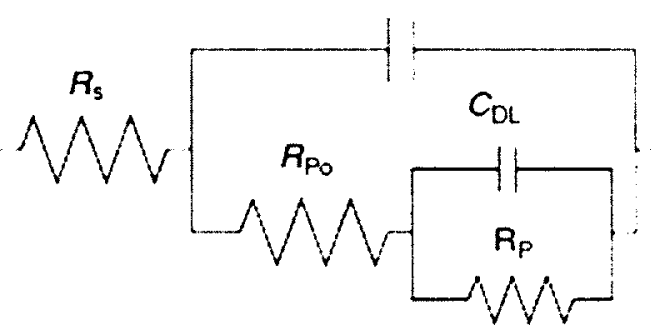

(c) $\quad C P E_{\text {caat }}$

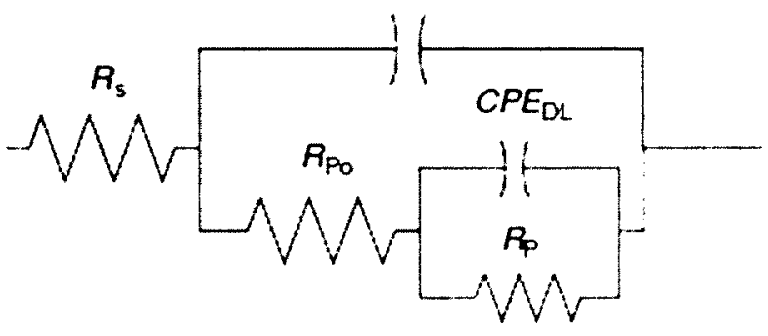

(d) $\quad C_{a}$

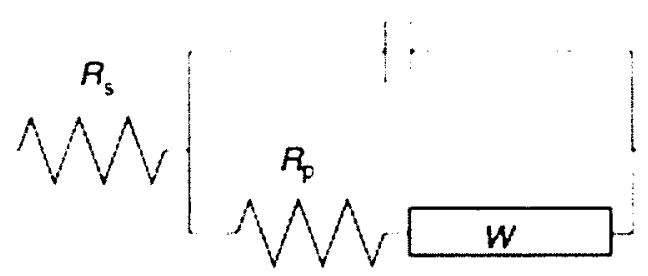

Figure 3-8 Circuits used to model EIS data of coatings on metals: (a) intact coating; (b) damaged coating where the electrolyte can reach the metal surface; (c) using constant-phase elements (CPE) instead of pure capacitors to model non-uniform distribution of the coatings and surface roughness; (d) addition of a Warburg element to model the impedance associated with diffusion and mass transfer processes [9].

Figure 3-8a represents an intact coating [9]. It consists of an equivalent circuit composed of a capacitor $\mathrm{C}_{\text {Coat }}$ representing the capacitance of the coating, in parallel with 
$R_{\text {Coat }}$ corresponding to the resistance of the coating, in series with $R_{s}$ corresponding to the resistance of the electrolyte/solution.

When the coating is damaged and the electrolyte can reach the metal surface, the shape of the Bode and Nyquist plots change, and the electrical equivalent circuit commonly used to model its behaviour is the one shown in Figure 3-8b. In this circuit, $C_{\text {Coat }}$ and $R_{s}$ have the same meaning as above, while $R_{P o}$ models the resistance of ionically conducting paths through the coating; $\mathrm{C}_{\mathrm{DL}}$ models the double-layer capacitance on the metal surface, and $R_{P}$ models the effective resistance of the corrosion process at the metal-electrolyte interface [9].

During the application of coatings, non-uniform distribution of the coatings and surface roughness lead to inhomogeneous distributions of the current. In such cases, ideal capacitors are not able to model experimental data, and EIS data are commonly modelled using constant-phase elements (CPE) instead of pure capacitors (Figure 3-8c) [9].

The circuit in Figure 3-8d includes a Warburg element. Warburg impedance is an element that models the impedance associated with diffusion and mass transfer processes. It is commonly used when the rate of diffusion of species through the pores of the coating or through corrosion products controls the corrosion rate, producing a distinctive $45^{\circ}$ tail in the low-frequency region of the Nyquist plot (Figure 3-10). This has been reported extensively in previous research papers $[28,37,42,43]$.

EIS data for the aforementioned circuits have been simulated and plotted, Figure 3-9. It can be seen from the graph, that these circuits produce data that very closely match typical EIS experimental data for coatings, Figure 2-8. 


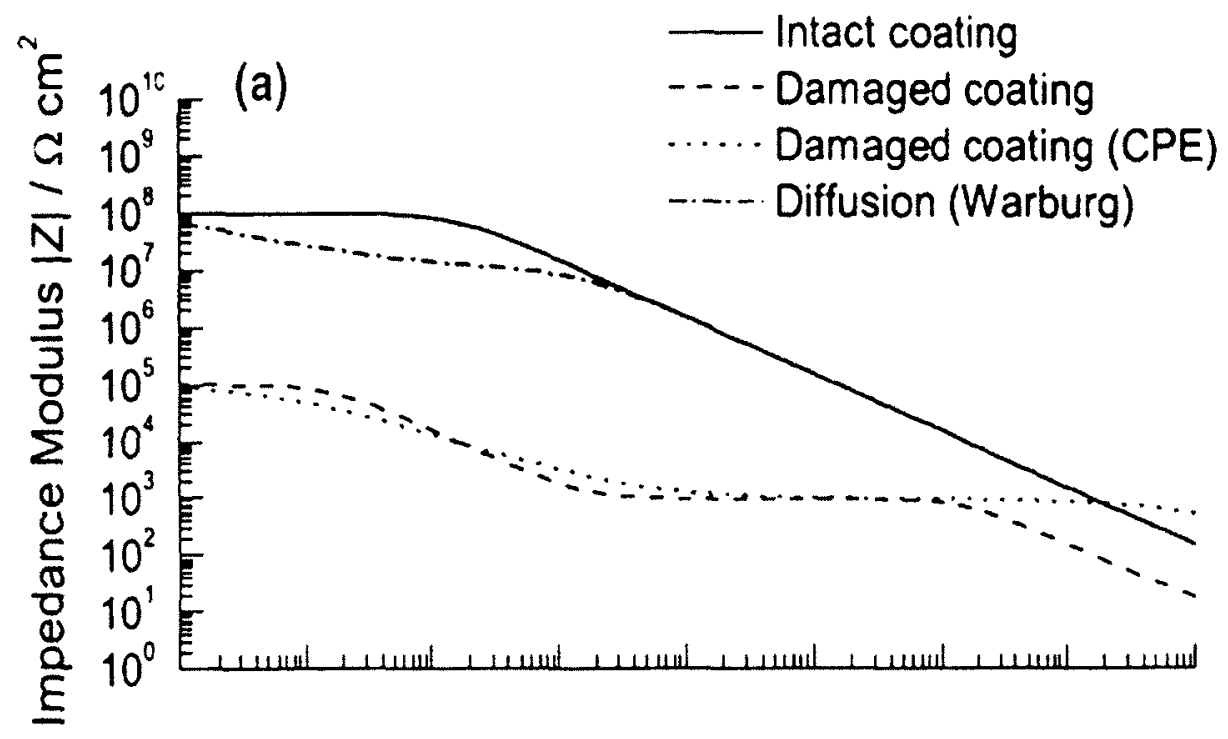

(b)

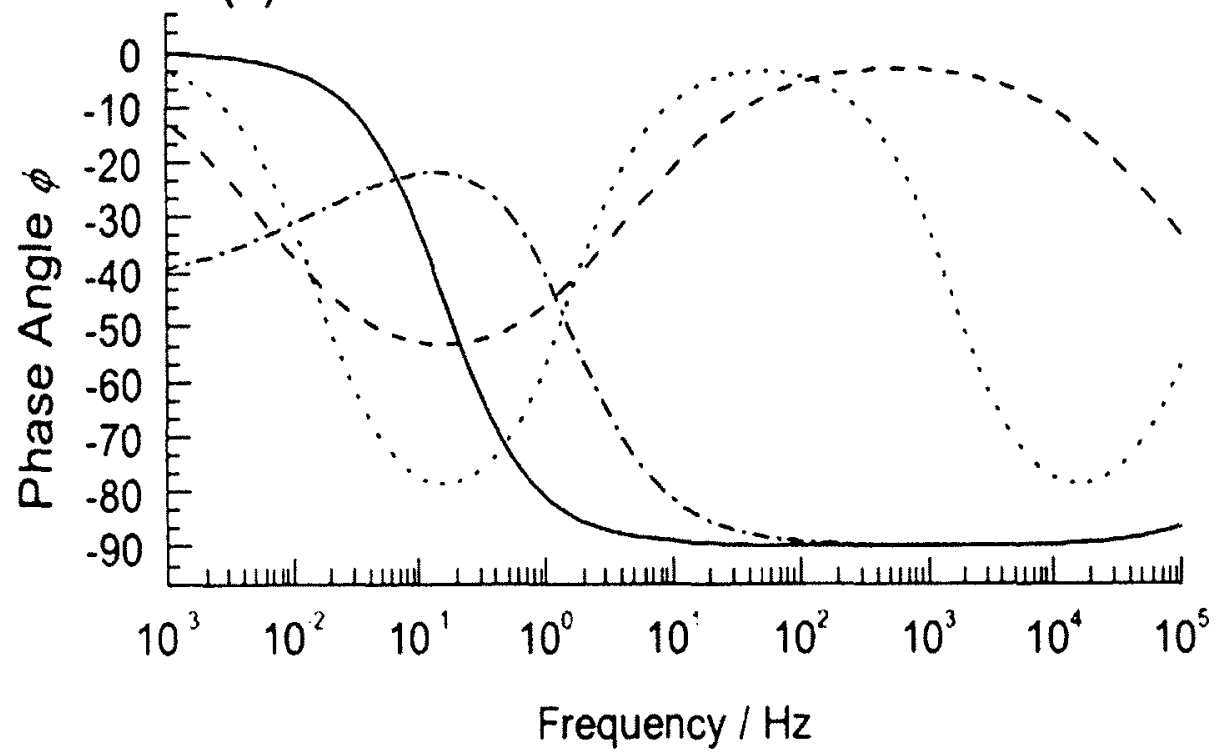

Figure 3-9 Simulated Bode plot using circuits represented in Figure 3-8 [9]. Note the increase in complexity in the plot with sample damage. 

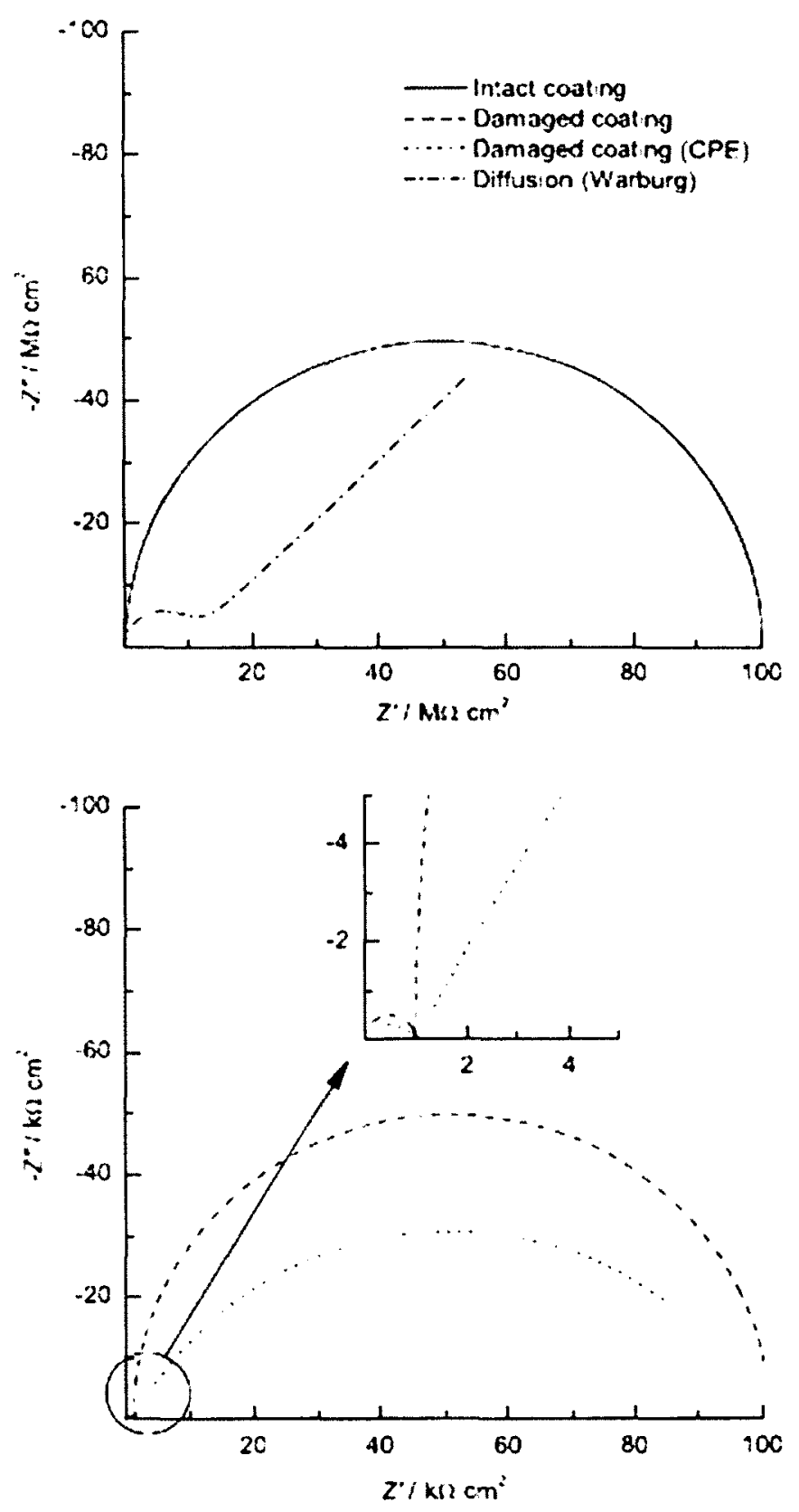

Figure 3-10 Simulated Nyquist plot using circuits represented in Figure 3-8 [9]. Note the appearance of a second semicircle with the sample damage.

The circuits shown are simplified models that, in many cases, can help to explain the experimental results. In many other cases, the EIS data are more complicated, and other more sophisticated electrical circuits have been proposed, such as additional $\mathrm{R}-\mathrm{C}$ sub-circuits [28, 29]; or several $\mathrm{R}-\mathrm{C}$ circuits in parallel, modelling different areas of a 
multilayer coating, which are not easy to interpret. In this situation, the circuit elements may not be easily correlated to real physical or chemical properties.

\subsection{Previous Studies on Aluminum Food/Beverage Containers}

Current test methods for the evaluation of coated aluminum food cans have various shortcomings. An industry standard two-year pack-test, where coated metal containers are filled with electrolytes and stored for two years, is subjective because it relies on visual examination. Other methods such as accelerating the corrosion by using acidic solutions or applying a voltage are not food product specific, but these do reveal weaknesses or potential failure areas in the coatings [30].

In one of the studies, several aluminum food cans were in storage for two years, after which EIS was used to evaluate the different can coatings. Described below are three methods used to evaluate the coatings: low frequency impedance (at $0.04 \mathrm{~Hz}$ ), break-point frequency $\left(\varphi=45^{\circ}\right)$ and percent ideal behavior [30].

The first method assumes a simplified Randles circuit, Figure 2-9, which has been extensively used in organic coatings evaluation. The low frequency impedance (at $0.04 \mathrm{~Hz})$ is approximately equal to the sum of the solution resistance, the coating resistance and the charge transfer resistance, and has been found to correlate with visually observable coating degradation.

The second method, the break-point frequency is also based on the use of a Randles circuit. It is identified when the phase angle of the system reaches $-45^{\circ}$, i.e., half way between restive and capacitive behaviour. In the modified Randles circuit, Figure 2-14, there are two break-point frequencies: the points where capacitive to resistive transitions occur, Figure 3-11. 

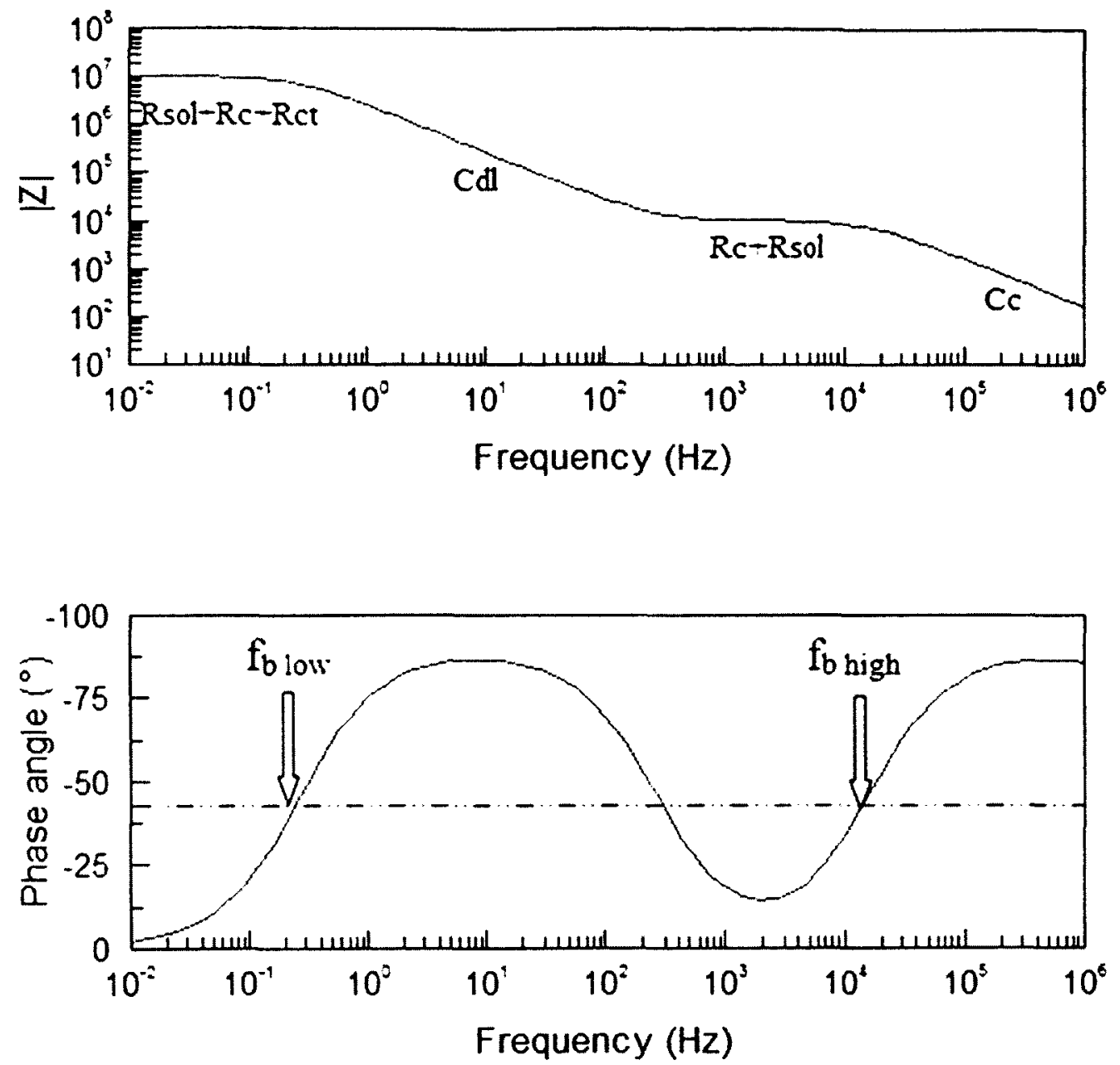

\section{Figure 3-11 Schematic representation of a Bode plot showing the break-point frequencies.}

The higher break-point frequency $\left(f_{b}\right.$ high $)$ occurs at a point where the resistive part of the impedance is equal to the pore resistance plus the solution resistance, while at the lower break-point frequency $\left(\mathrm{f}_{\mathrm{b} \text { low }}\right)$, the resistive part of the impedance is equal to the charge transfer resistance plus the pore resistance and solution resistance. The term $f_{b}$ high corresponds to changes in the coating characteristics, while $f_{b}$ low is related to the metal interface, coating and electrolyte. The higher break-point frequency is not always measurable and so the low break-point frequency is used. An increase in $f_{b}$ (high and/or 
low) over time typically means a decrease in the specific resistance of the coating, or an increase in the delaminated area ratio $[9,29-34,46]$.

In the third method, the 'percent ideal' is the ratio of the area under the Bode curve to the area under the ideal, purely capacitive curve as shown in Figure 3-12 [30]. The advantage of the third method is that data from the entire frequency range is used, whereas in the first two methods, only a small portion of the spectrum is used.

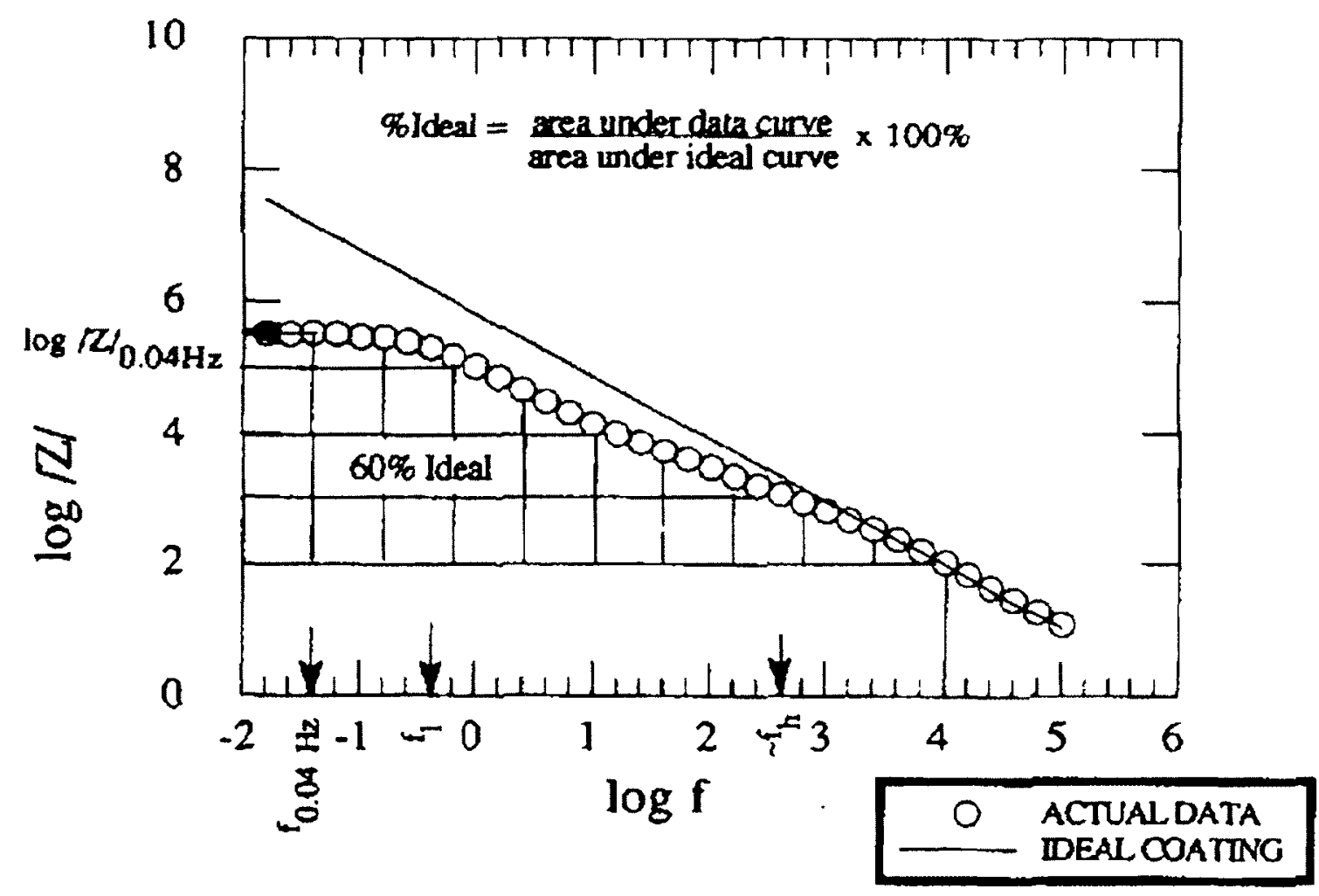

Figure 3-12 Bode plot representing the \% ideal method used to represent EIS data [30].

All three methods mentioned above can be used to rank the coating performances. The first method, low-frequency impedance is the easiest to use since it is easily obtainable from the Bode plot. The second method, break point frequency, is not always easily or accurately obtainable [30]. 
In another two-part study $[31,32]$, the aim was to develop EIS as a method for quantifying coatings on $\mathrm{Al}$ beverage containers. The focus of part 1 was to find a parameter that could be obtained quickly to evaluate performance of coated $\mathrm{Al}$. In part 2 , this parameter was used in a statistical study to evaluate multiple samples with different coatings.

The impedance response of uncoated $\mathrm{Al}$ was compared with that of coated $\mathrm{Al}$ samples (with varying coating thickness) with and without an intentional defect. This initial assessment enabled differentiation between the part of the EIS spectrum associated with the coating and that associated with the solution-oxide-metal interface.

The defect was a $0.1 \mathrm{~mm}$ diameter scribe on the surface of a coated aluminum sample. Before the defect, the EIS spectra were purely capacitive. After producing the defect, impedance values below $10 \mathrm{~Hz}$ decreased with time and more the phase angle plots were observed to increase in complexity [31] (Figure 3-13).
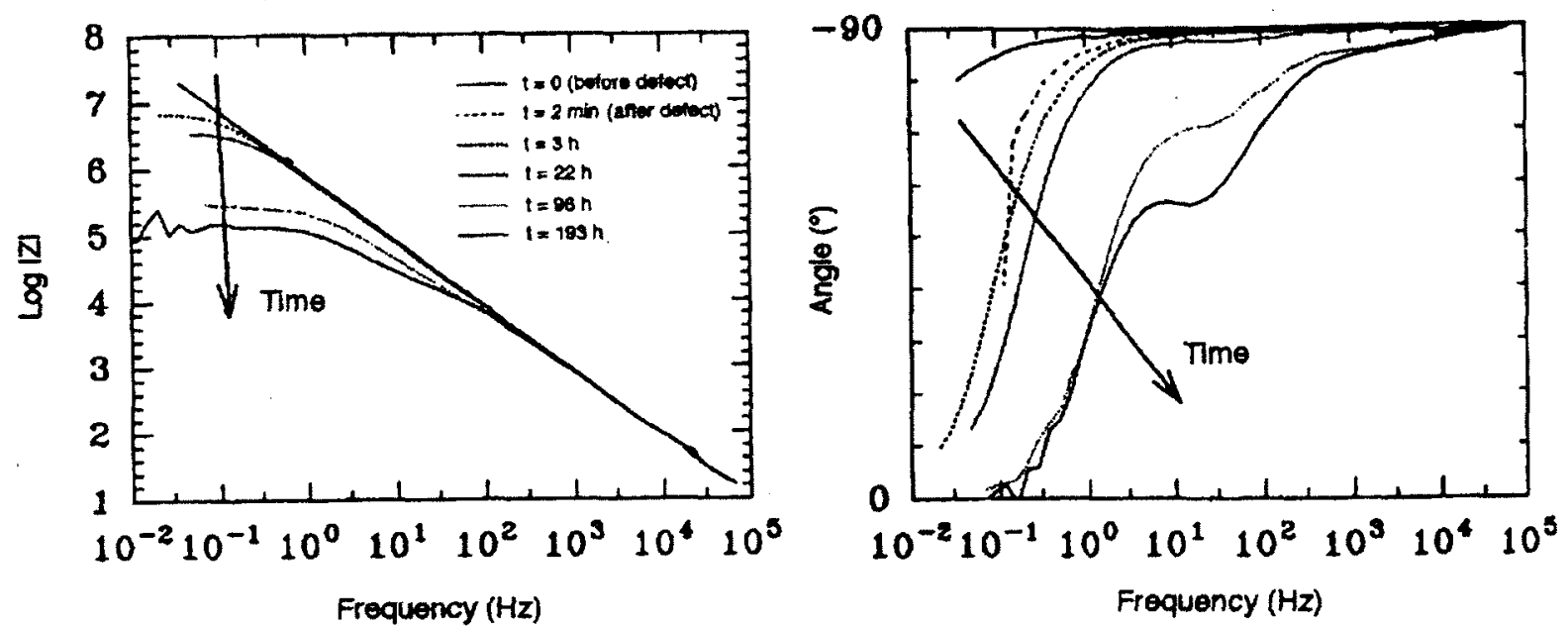

Figure 3-13 Impedance spectra for coated aluminum before and after introducing a defect [31]. 
By comparing the phase plots in Figure 3-14, the high frequency $\left(10^{2} \mathrm{~Hz}-10^{3} \mathrm{~Hz}\right)$ is believed to contain information about the coating, $C_{c}$ and $R_{\text {pore }}$, and the low frequency $(\sim 5 \mathrm{~Hz})$, contains information on the aluminum oxide and the metal surface interactions [31].

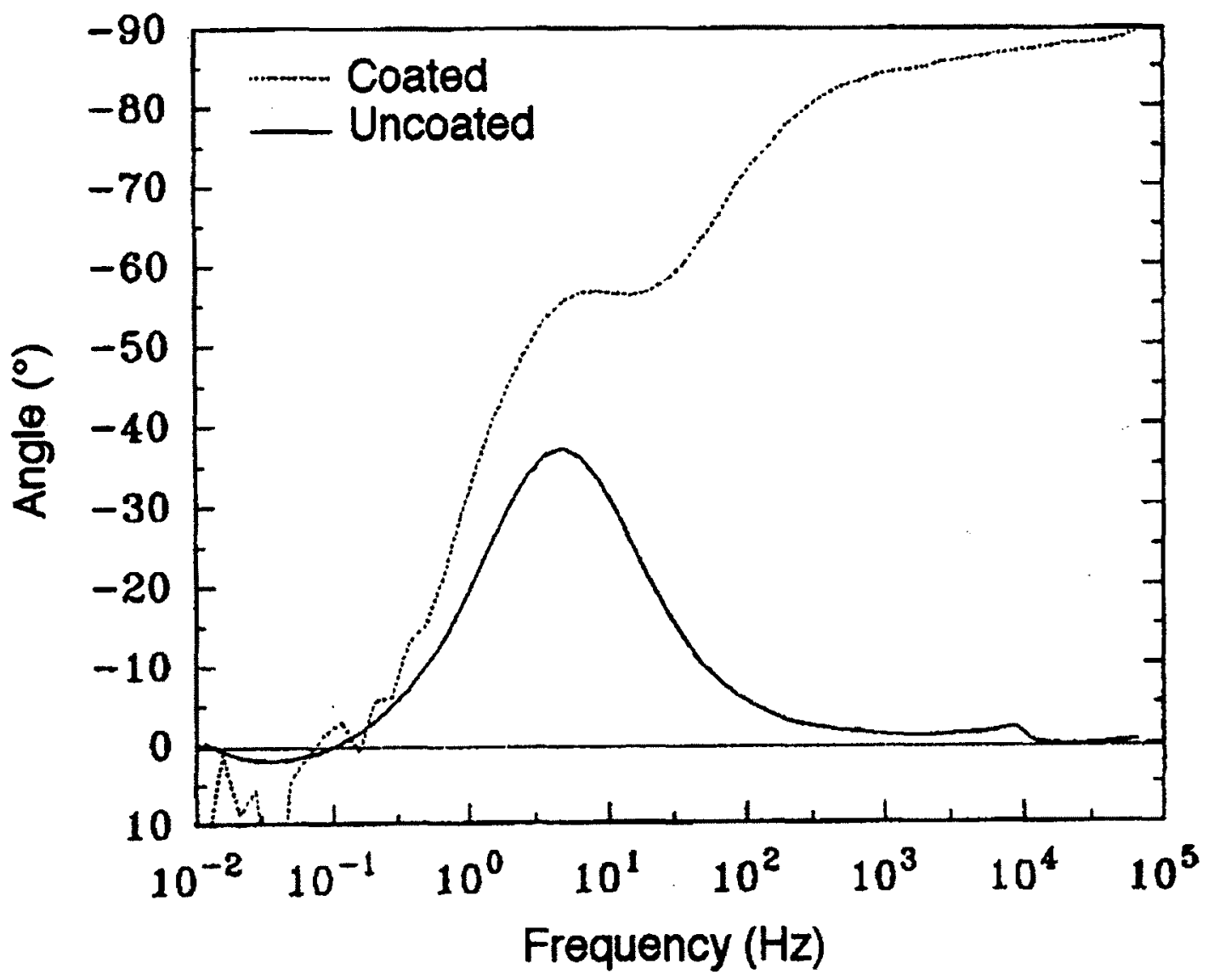

Figure 3-14 Bode phase-angle plots for uncoated and coated (with defect at 193h) aluminum [31]. Note the appearance of positive phase angles at the low end of the frequency spectrum which is discussed in section 5.3 and chapter 6.

EIS spectra for coated and uncoated aluminum samples were also collected at several DC polarization potentials. For the samples held at a potential in the transition region between active and passive ranges, there was scatter in the results and positive 
phase angles at frequencies below $1 \mathrm{~Hz}$. This scatter was attributed to current fluctuations caused by metastable pits forming due to the applied DC voltage.

The proposed model for the corroding coated Al metal is shown in Figure 3-15 [31].

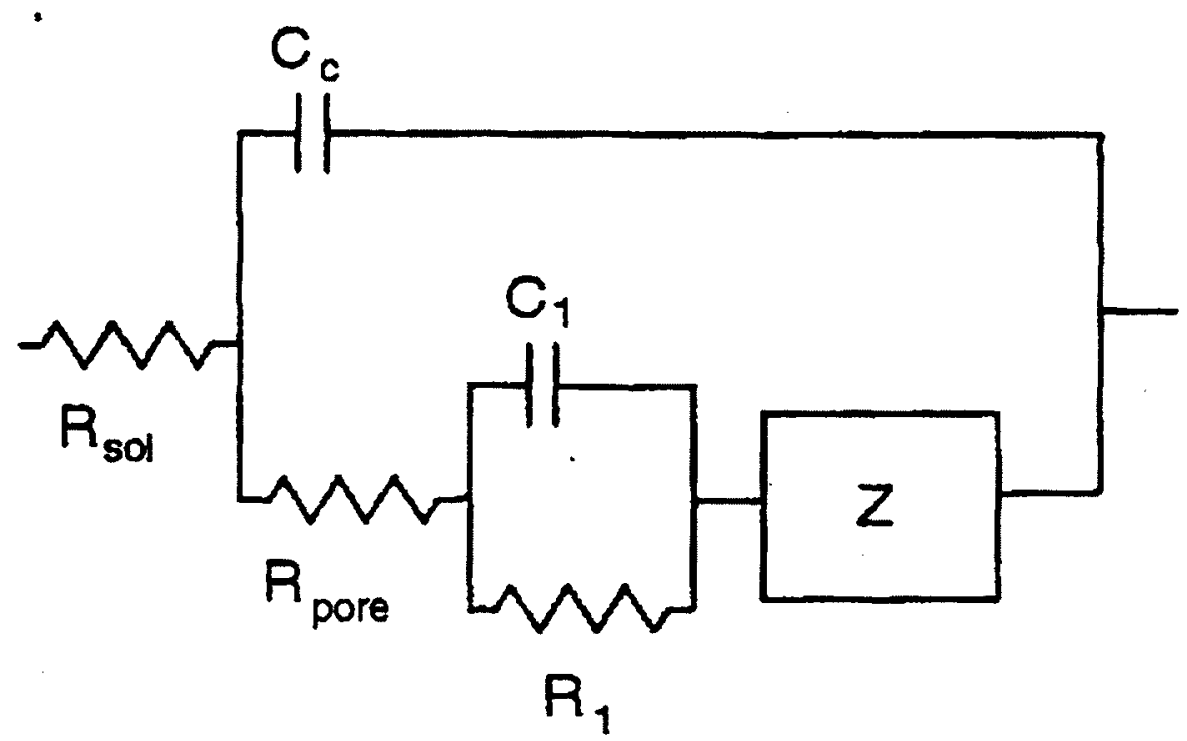

where

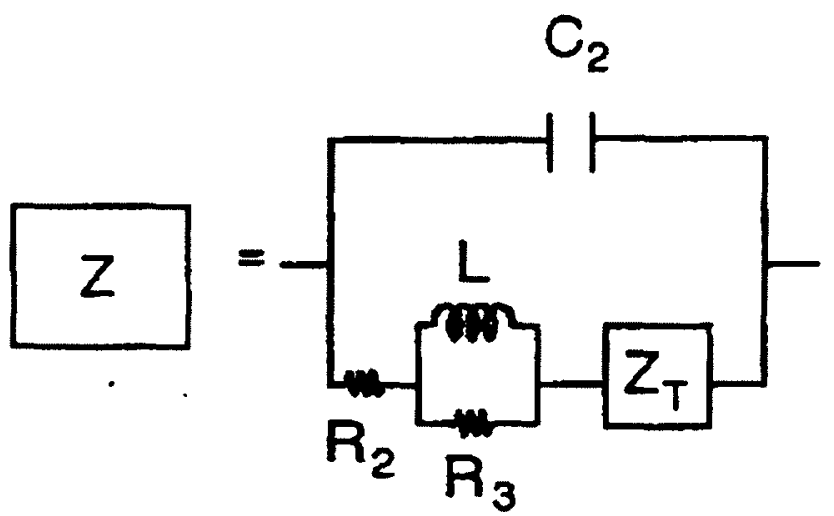

Figure 3-15 Circuit model proposed to represent coated aluminum in chloride containing aqueous media [31].

By fitting data to the model, $\mathrm{C}_{\mathrm{c}}$ values were obtained and plotted against time. There was no direct correlation between the performance of the coatings, after 5 months of exposure, and the calculated $\mathrm{C}_{\mathrm{c}}$ values. Changes in this parameter only occurred when 
degradation was severe, such as in the case of the sample with intentional defect, and there was still no correlation between visual observations and changes in $\mathrm{C}_{\mathrm{c}}$. Therefore, $\mathrm{C}_{\mathrm{c}}$ was not a good measure of coating performance [31].

In a similar manner, EIS data were fitted to the model in Figure 3-15 and $R_{\text {pore }}$ values were estimated and plotted against time. Changes in $R_{\text {pore }}$, over a few days, were consistent with visual changes observed after 5 months of exposure. On the downside, such a technique would be time consuming and hard to execute for a large sample population.

To overcome this obstacle, the low frequency impedance, at $0.05 \mathrm{~Hz}$, was used to rank coatings. The impedance at this frequency included the response of the coating and response of the oxide. Although its value did not give information about the details of coating degradation mechanisms, it is easily obtainable and most importantly it agreed well with visible corrosion observed after a 5 month period of exposure [31].

\subsection{Localized EIS Techniques}

Conventional electrochemical techniques do not provide quantitative information on the delamination of polymeric coatings from metallic substrates, and they cannot provide information from a local area. It is particularly difficult to analyze the delamination of organic coatings in the presence of defects. Conventional electrochemical impedance spectroscopy provides little information on the shape and the extent of the delaminated area [33].

The principles of localized electrochemical impedance spectroscopy (LEIS) are similar to those employed in traditional bulk EIS, in that a small sinusoidal voltage perturbation is applied to a working electrode sample and the resulting current is 
measured to allow the calculation of the impedance. However, rather than measure the bulk current, a small electrochemical probe is scanned close to the surface, measuring the local current in the electrolyte [25].

For the study of defects existing in coatings, scanning electrode techniques are increasingly being used due to their ability to provide information with spatial resolution. Novel information concerning the electrochemical and degradation processes at defects in coated metals is being obtained with the scanning vibrating electrode technique (SVET), the scanning Kelvin-probe (SKP) method, localized electrochemical impedance spectroscopy (LEIS), and scanning electrochemical microscopy (SECM) $[25,50-55]$. They are able to provide map-like surface contour diagrams demonstrating the variations of potentials, impedance or current on the surface, for a specific frequency. Practically, this can only be done for a small area, and is not readily useable for samples that size of aluminum cans.

Such techniques might be useful to find surface variations or defects, for example before and after immersion, but they have many disadvantages: too many variables; they are expensive; they are time consuming [50-55]. A technique that examines larger sample areas is preferable. Owing to their drawbacks, localised EIS techniques have not been used in the current research.

\subsection{Fluorescent Coatings}

It is appropriate to mention a new tool being used to indicate corrosion of coated metals, although we have not employed this technique in our present work. Besides serving as a protective barrier, organic coatings can also be employed to serve as a corrosion sensor by incorporating indicator molecules into coating formulations. Due to interactions of 
indicator molecules with ions generated during corrosion, the onset of corrosion can be detected by a change in color or fluorescence of the indicator molecules. This sensing method is both non-destructive and in-situ [18].

By using acid-base indicators which change their color or fluorescence depending on $\mathrm{pH}$, detection of the localized $\mathrm{pH}$ changes at anodic and cathodic sites of corrosion is possible for aluminum samples. Such indicators are usually weak organic acids or bases and their dissociated form has a different color or fluorescence than their un-dissociated form.

In a study [18], an aluminum corrosion-sensing coating was prepared, and when the sample started to corrode, a color change was indicated by a color change in the coating. Although the $\mathrm{pH}$-sensing approach seemed to work well in clear acrylic and polyurethane coating systems, it has proven to be challenging in epoxy coatings due to the reactivity of its components. In an attempt to use $\mathrm{pH}$-sensitive molecules in an epoxyprimer coating applied onto an aluminum plate, the indicator became prematurely fluorescent. This phenomenon can be ascribed to the premature chemical reaction between the indicator and one of the coating precursors; thus, it would be very difficult to use the acid-base indicator approach in epoxy-based coatings for corrosion detection [18].

An ideal corrosion indicator for aluminum would not possess any functional groups that could be ionized and would not react with the epoxy components in such a way as to alter their fluorescent properties. It would also preferably employ a "turn-on" fluorescence which means that the initially non-fluorescent indicator would become highly fluorescent at the onset of corrosion, before any obvious sign of metal corrosion. 
FD1, a new molecule, has proven to be a successful corrosion indicator for aluminum alloys [18]. It is able to sense localized decreases in $\mathrm{pH}$ at the anodic site of corrosion in epoxy coatings. The onset of pitting corrosion was easily and nondestructively detected by simply shining a handheld UV lamp on the aluminum panels coated with the FD1-containing epoxy coating [18].

Figure 3-16 shows images of an aluminum sample coated with a FD1-containing clear epoxy coating, which changes color after exposure to $3.5 \% \mathrm{NaCl}$ solution. This approach is easier to observe and is much more useful and practical since it is easier to detect small areas that fluoresce (against a background that does not) than to observe a slight decrease in overall fluorescence [18].
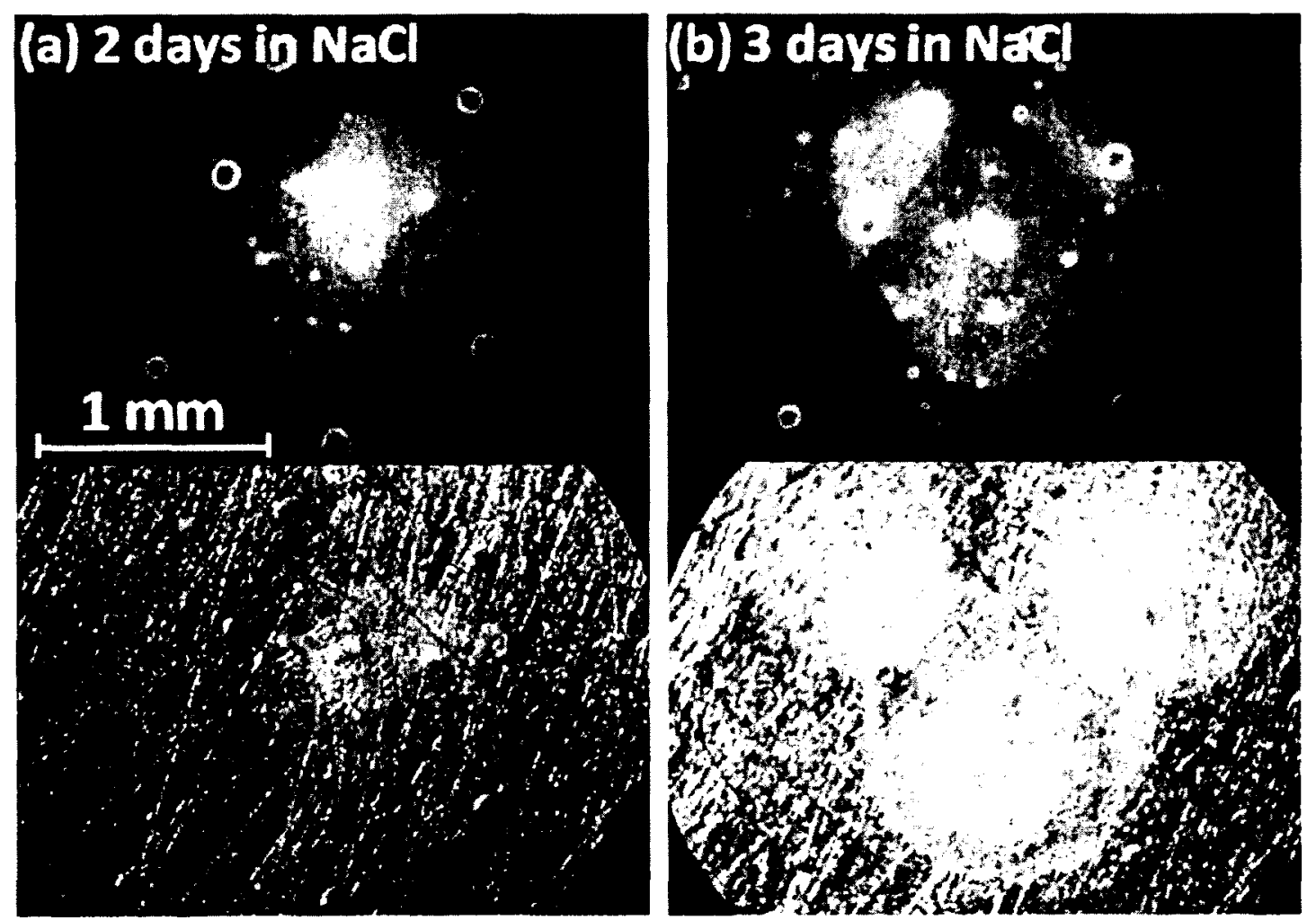

Figure 3-16 Images of an aluminum sample coated with a FD1-containing clear epoxy coating after (a) 2 days and (b) 3 days of exposure to $3.5 \% \mathrm{NaCl}$ solution. Top row: digital camera images taken through the microscope eyepiece under UV light. Bottom row: images of the same area taken through the microscope eyepiece under natural light [18]. 


\subsection{Summary}

For the evaluation of metal coatings, a variety of methods have been utilized. The use of conventional testing methods is very time consuming and only provides qualitative or semi-quantitative data. It has been shown that there is no direct correlation between such methods and corrosion detection; they measure the quality of coating application techniques more so than the performance of the coatings themselves.

Electrochemical impedance spectroscopy is one of the many techniques used for evaluating metal coatings. EIS data are complex. For interpreting such data, parameters such as break-point frequency, low-frequency impedance and coating capacitance $\left(\mathrm{C}_{\mathrm{c}}\right)$ have been used. Such parameters can only serve to distinguish between intact and penetrated coatings; they provide no information on the onset of corrosion or the corrosion process of samples. However, in one of the studies, it was concluded that changes in $R_{\text {pore }}$ over 8 days can be associated with corrosion that is visible after 5 months, thereby providing an early detection method.

Equivalent circuit analysis has also been used to interpret EIS data, but this technique can require very complex models which sometimes have no physical meaning.

Recently, localized EIS techniques have been developed. Such techniques are complicated and expensive when compared to the conventional EIS method. They could only serve to provide information on small specific areas of the samples, and perhaps only at specific frequencies. For doing assessments of large samples, localised techniques are impractical.

The aim of this research is to provide a rapid performance evaluation method for both existing and new coatings, used to protect aluminum containers. None of the 
aforementioned testing methods provide any signatures of corrosion or easily obtainable methods for early detection of the onset of corrosion. It is apparent, however, that EIS has not been fully explored in the area of testing food and beverage containers. The current research provides indications and information that has not been previously used to test these containers. From EIS and polarization data, early signatures of corrosion will be recorded and used as an early detection method for a quick and comprehensive evaluation of coatings. 


\section{Chapter 4. Experimental Setup and Equipment Used}

\subsection{Introduction}

This chapter provides the details of the experimental procedure followed and all the equipment used for the collection of EIS and potentiodynamic data. This includes the different potentiostats, the softwares used, as well as the different electrochemical cells.

\subsection{Coated Aluminum Samples}

Coated aluminum samples were received as flat sheet panels and converted can ends. The formulation of the coatings was not provided as it is proprietary. Converted can ends, with two different coatings were available for testing; they were labelled as coating 1 and coating 2. All the flat sheet panels had coating 1 applied to the surface. The difference between the coatings is that coating 1 is epoxy-free while coating 2 is not. The aluminum samples are coated on both sides, the beverage side (interior coating, $\sim 8$ microns thick) and the public side (exterior coating, $\sim 4$ microns thick).

\subsection{Experimental Procedure}

During service, the interior coating of the aluminum samples is exposed to deaerated acidic solutions with an approximate $\mathrm{pH}$ of 3. As explained in section 3.3.1, the aluminum oxide is stable in the $\mathrm{pH}$ region of $4 \sim 9$. The lower the oxygen content, the slower is the corrosion rate [15].

It was important to explore the effects of varying the $\mathrm{pH}$ and oxygen content on the performance of the coated aluminum samples. This was achieved with the use of aerated and deaerated solutions. EIS scans were continuously recorded until sample 
failure. In some cases experiments ran for as long as 2500 hours. All EIS experiments were performed with an $\mathrm{AC}$ excitation voltage of $20 \mathrm{mV}$ with the exception of the very high-impedance polymer experiments which were run with an $\mathrm{AC}$ voltage of $100 \mathrm{mV}$. All potentiodynamic data were collected at a scan rate of $10 \mathrm{mV} / \mathrm{s}$ [34].

Sodium chloride solutions (MSDS\# 7560-1, CAS\# 7647-14-5) and hydrochloric acid solutions (UN\# 1789, CAS\# 7647-01-0) were utilized for the testing of the coated aluminum samples since they cause rapid corrosion and pitting of aluminum when the protective lacquer coating is breached; discussed in section 3.3.1. The coated samples were investigated under the following conditions:

- Aerated $1 \mathrm{M} \mathrm{NaCl}(\mathrm{pH} 8)$;

- Aerated $0.1 \mathrm{M} \mathrm{NaCl}(\mathrm{pH} 8)$;

- Aerated $\mathrm{HCl}(\mathrm{pH} \mathrm{0.1);}$

- Aerated $\mathrm{HCl}(\mathrm{pH} 2.6)$;

- Deaerated $1 \mathrm{M} \mathrm{NaCl}$ (using dry ice);

- Deaerated $1 \mathrm{M} \mathrm{NaCl}$ (using $\mathrm{N}_{2}$ gas);

- Deaerated $1 \mathrm{M} \mathrm{HCl}$ (using dry ice).

Under each of the aforementioned conditions, for the verification of the results, experiments were performed several times. The $\mathrm{pH}$ of the solutions was measured before and after deaeration with the use of 'Orion 2 Star' bench-top pH meter. It was found that deaerating the solutions did not change the $\mathrm{pH}$. Besides recording EIS data, images of intact and damaged samples were produced with the use of a 'JEOL-JSM840' scanning electron microscope (SEM) for comparison purposes. 


\subsection{Data Acquisition Systems}

Initially, two instruments were used for the gathering of EIS spectra of aluminum-foil samples, high-impedance polymers and beverage-can coated aluminum samples: a Solartron 1260 impedance gain/phase analyzer (referred to as Solartron 1), and a Gamry PC4/300 (referred to as Gamry 1). Later in the investigation, a Solartron 1287 potentiostat with a Solartron 1255 gain/phase analyzer (referred to as Solartron 2) became available, as well as a second Gamry PC4/300 (referred to as Gamry 2). Solartron 2 and Gamry 2 were used for the purpose of data comparison and verifications of results previously obtained on Solartron 1 and Gamry 1, and for performing DC experiments.

The analyzers do not measure impedance directly, but rather they measure $\mathrm{V}$ and I and compute the impedance of the item under test [35]. All of these instruments are generally referred to as potentiostats. All high-performance EIS systems use a fourterminal connection scheme. The four leads that connect to the cell under test are grouped into two pairs. One pair of leads, 'current-carrying leads', conduct the current between the cell and the potentiostat, and 'sense leads', measure the voltage across two points in the cell [36].

\subsection{Noise Elimination in Solartron Instruments}

To reject any external electrochemical noise from the EIS data, an integration function is available in the Solartron instruments. Integration time is the period over which the analyzer measures the input signals. The duration of this period determines the noise rejection ability of the analyzer. Defined in seconds, the time is rounded up or down to cover a whole number of cycles. Integration is simply averaging of repeated cycles at the 
same frequency with 1 cycle at $1 \mathrm{MHz}$ taking a millionth of a second and 1 cycle at $1 \mathrm{mHz}$ taking 1000 seconds. For example, if averaging (integration) was set as 1000 cycles, it would take $1 / 1000$ th of a second to do the $1 \mathrm{MHz}$ measurement and over 11 days to do 1000 cycles at $1 \mathrm{mHz}[35]$.

In many cases integration beyond 1 second is not necessary, but in some cases where the value of impedance gets high resulting in a small current response it may be necessary as the signal to noise ratio of the current signal gets lower. The idea behind the various auto integrations is to only use as much averaging as necessary to get a certain statistical distribution in the measurements. Therefore to reject noise from the measurements it is necessary to increase the integration time. The measurements are increasingly more stable as integration time is increased, but of course the experiment will take longer to run [35].

Several integration times and cycles were tested, and it was found that autointegration function, "Long on V1", provided the shortest measurement times for spectra that were indistinguishable on $\log |\mathrm{Z}|$ versus $\log$ frequency plots. In this mode, the system aims for a standard deviation of $\pm 1 \%$ on analyzer input [35].

\subsection{Noise Elimination in Gamry Instruments}

In the Gamry systems, two noise optimization options are available: speed and accuracy. Speed is the appropriate selection when the cell stability is poor and a spectrum must be measured rapidly, or the system impedance is low and well defined. Accuracy is the appropriate selection when the cell impedance is high or the electrochemical system is noisy [37]. Both options were tested and both options produced acceptable results (i.e., 
indistinguishable spectra on $\log |Z|$ versus $\log$ frequency plots); however, the accuracy option was utilized [37].

\subsection{Gamry and Solartron Software}

With the different potentiostats, different computer programs were utilized to perform the measurements and data analyses. In the case of the Solartron instruments, for performing the EIS experiments, ZPlot (3.2c) was used, and for the viewing and analysis of the data ZView (3.2d) was used. For the Gamry systems, GamryFramework (4.35) was used for the collection of the data while EchemAnalyst (5.3) was used for the data viewing and analysis. For performing the DC experiments and cyclic voltammetry on Solartron 2, CorrWare (3.2c) and CorrView (3.2d) softwares were utilized.

As shown later in Table 5-1 (section 5.2.6) and Table 5-2 (section 5.4.2), to check the accuracy and precision of the potentiostats, values of known circuit elements were estimated by fitting EIS data to equivalent circuit models using non-linear least squares.

\subsection{Electrochemical Cells}

\subsubsection{Vertical Corrosion Cell (VC)}

The Vertical Cell, shown in Figure 4-1, is a Tait cell (EG\&G model K03074) purchased from Princeton Applied Research. It is an electrochemical cell that can be used to investigate the properties of coatings and paints exposed to aggressive solutions. It is comprised of a glass cylinder with two Hastelloy metal end caps and o-ring seals. The assembly is bolted together forming a leak-tight cell. The cell used in this investigation exposes an interior circular sample area of $34.0 \pm 0.5 \mathrm{~cm}^{2}$. Two flat rubber o-rings ensure a 
tight seal between the glass cylinder and the sample, and the glass cylinder and the topend cap. From the top-end cap two metal electrodes are suspended: a counter electrode and a reference electrode. The counter electrode has a wide surface area covering almost the entire cross section of the glass cylinder and is placed $10 \mathrm{~mm}$ above the surface of the coating (i.e., the working electrode) being tested. The large surface area of the counter electrode is required to provide a uniform current to the entire surface area of the coating placed underneath, ensuring that measurements are accurate and representative of the whole surface of the coating. The reference electrode is suspended through a small hole at the centre of the counter electrode, and its tip is placed $5 \mathrm{~mm}$ away from the surface of the coating. Both electrodes are made from Hastelloy steel, which is a special alloy used in highly corrosive environments, where corrosion resistance is required [38].

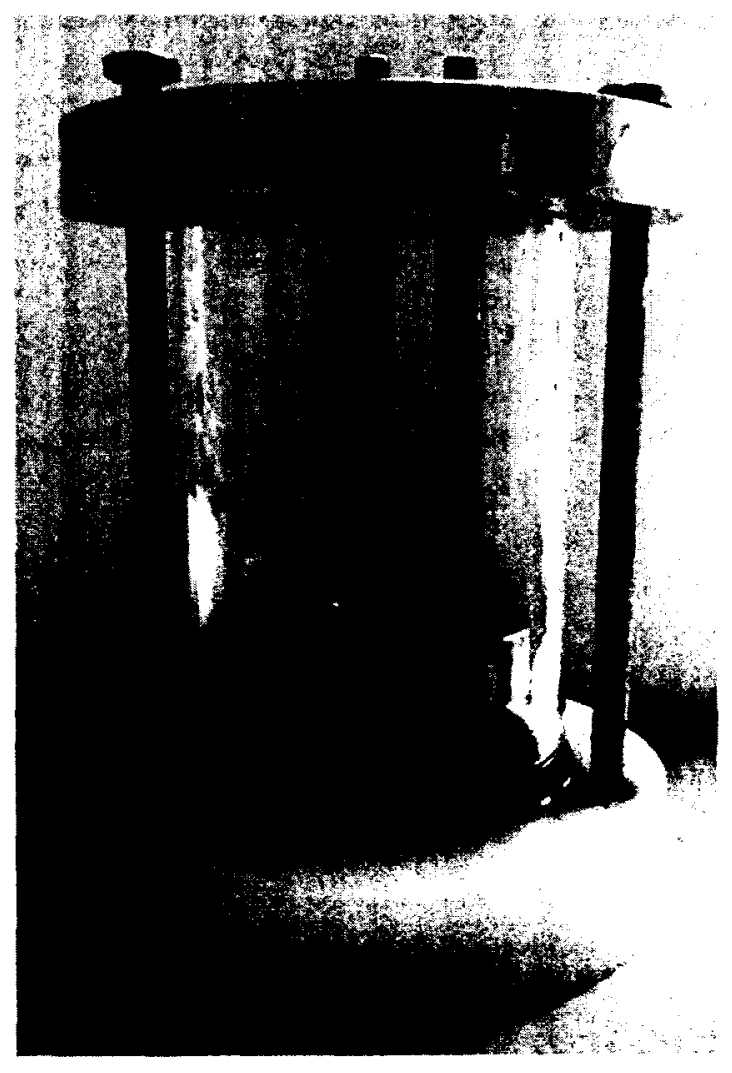

Figure 4-1 Vertical corrosion cell. The sample is shown under the black O-ring at the bottom of the glass cylinder. The sample diameter is $6.35 \mathrm{~cm}$. 


\subsubsection{Horizontal Corrosion Cell (HC)}

The Horizontal Cell, shown in Figure 4-2, is a "Flat cell kit" (EG\&G model K0235) purchased from Princeton Applied Research. It is an electrochemical cell that can also be used to investigate the properties of coatings and paints exposed to aggressive solutions. It is comprised of a Pyrex ${ }^{\mathrm{TM}}$-glass cylinder body with polypropylene end caps. The assembly is bolted together forming a leak-tight cell. The Horizontal cell used in this investigation encloses an interior circular sample area of $1.0 \pm 0.1 \mathrm{~cm}^{2}$. A flat rubber oring ensures a tight seal between the polypropylene end caps and the sample. Two electrodes are suspended through the top, a counter electrode and a reference electrode; both electrodes are made from graphite [39].

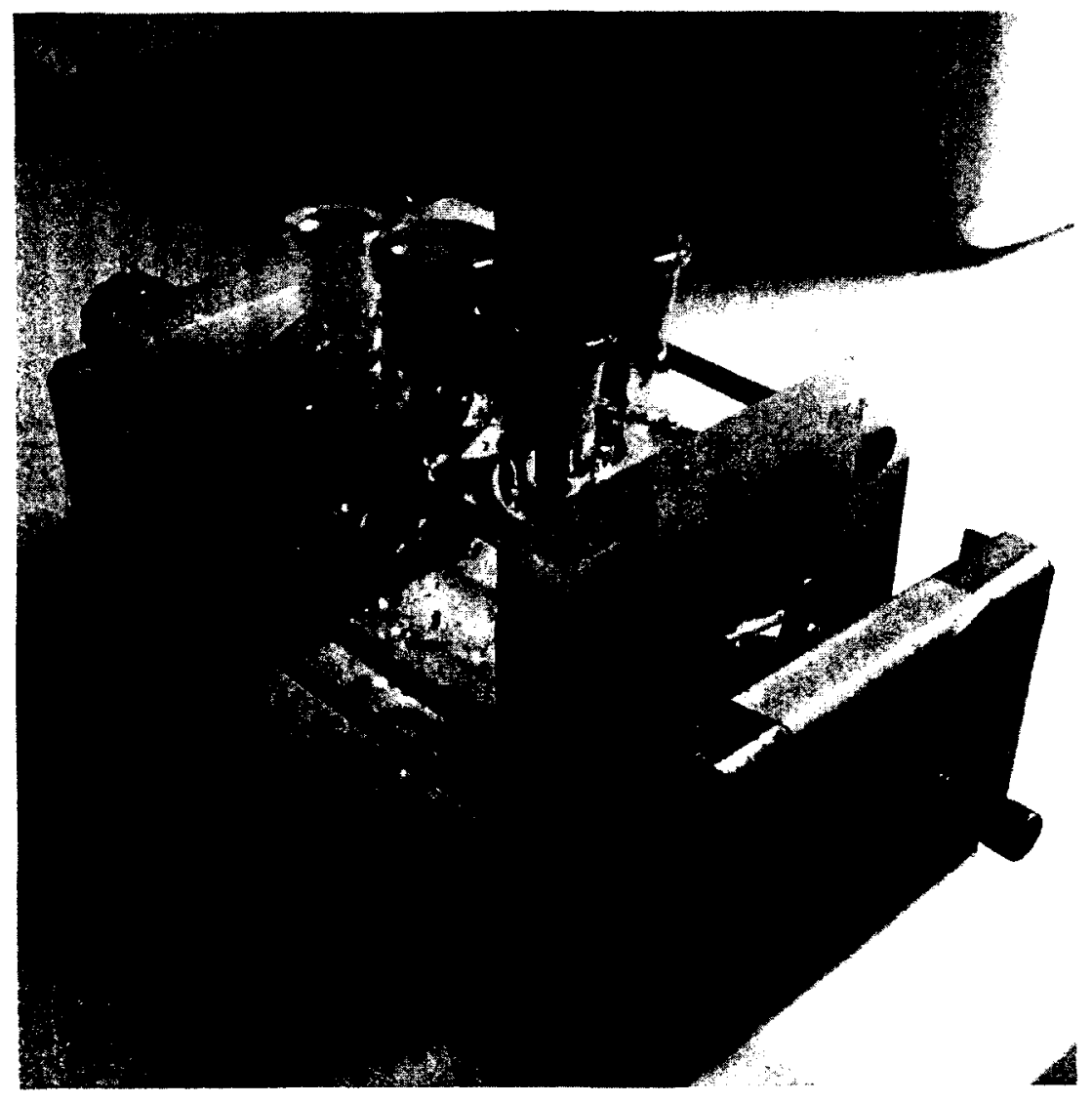

Figure 4-2 Horizontal corrosion cell. The volume of the cell is $250 \mathrm{ml}$ and the working electrode area is $1 \mathrm{~cm}^{2}$. 


\section{Chapter 5. Preliminary Experiments}

\subsection{Introduction}

This chapter presents the preliminary investigations performed prior to performing longterm experiments on the coated-aluminum samples. As shown in Chapters 2 and 3, intact coatings have very high impedances that might be beyond the measuring capabilities of the potentiostats. This issue was investigated, and the first section presents the tests and analysis performed to ensure that the EIS measurement instruments at hand are capable, functional and consistent. This was followed by EIS testing of kitchen-aluminum foil and high-impedance epoxy polymer to obtain a baseline for chemically-active and chemically-inert systems. Finally, the effect of varying the sample size and electrolyte concentration, on EIS data, was investigated.

All EIS experiments were performed with an AC excitation voltage of $20 \mathrm{mV}$ with the exception of the very high-impedance polymer experiments which were run with an $\mathrm{AC}$ voltage of $100 \mathrm{mV}$. It is customary to run EIS spectra from high frequency down to low frequency. When plotting data, the high frequency is normally on the right-hand side of the horizontal-axis, so that all experiments proceed from right to left on graphs. All potentiodynamic data were collected at a scan rate of $10 \mathrm{mV} / \mathrm{s}$.

\subsection{Instrumentation Analysis}

\subsubsection{Introduction}

This section discusses and explains the tests and analysis performed to ensure that the instruments used to perform the EIS measurements were properly functioning and producing meaningful results. 
Prior to investigating the EIS spectra of aluminum foil and coated aluminum samples, the following performance parameters were investigated:

- Cable effects;

- Limits of operation of the machines;

- Performance at high and low frequencies using high impedance coatings as well as basic circuit elements;

- Comparison of the Gamry instruments;

- Comparison of the Solartron instruments;

- The effect of external noise on EIS spectra.

\subsubsection{Cable Effects}

Before investigating the performance limits of the potentiostats and their consistency, the integrity of the experimental set-up was first tested. Several sets of connection cables for each system were available. The cable connecting the instrument and the cell, and placement of the leads connecting to the cell can have a major effect on EIS system performance. The term mutual inductance describes the influence of the magnetic field generated by the current carrying leads on the sense leads. Mutual inductance can limit the ability of an EIS system to make accurate measurements of low impedances at high frequencies. In essence, the current carrying leads are the primary of a transformer and the sense leads are the secondary. The AC current in the primary creates a magnetic field that then couples to the secondary, where it creates an unwanted AC voltage. Mutual inductance errors are more significant with lower cell impedances and higher frequencies $[36]$. 
To test the integrity of the cables, EIS data were collected for high impedance (see below) and low impedance systems (see fuel cell experiments in section 5.2.5). For the high impedance testing, a high-impedance coated aluminum sample was placed in the EIS cell with tap water as the electrolyte. This is a stable system, which always gives highly reproducible results, and any changes in EIS spectra when the cables are changed are due to differing electrical responses of the cables and are not due to changes in cell corrosion or chemistry. First, Solartron 1 and Solartron 2 were tested and their two cables were interchanged. There were no significant differences in the EIS spectra and all the data were consistent: Figure 5-1 and Figure 5-2.

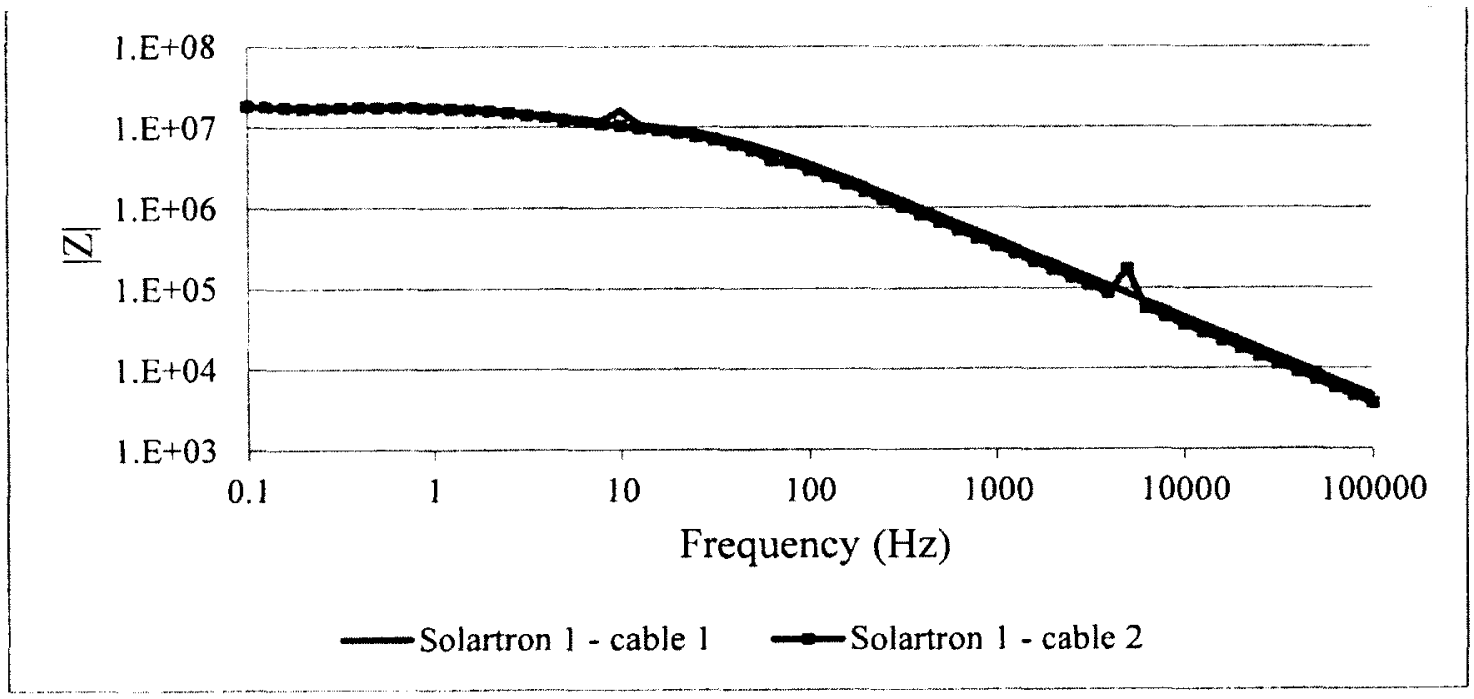

Figure 5-1 Impedance $(\Omega)$ measurements $\left(\mathrm{HC}, 1.0 \pm 0.1 \mathrm{~cm}^{2}\right)$ for coated aluminum using Solartron 1 with different cables. 


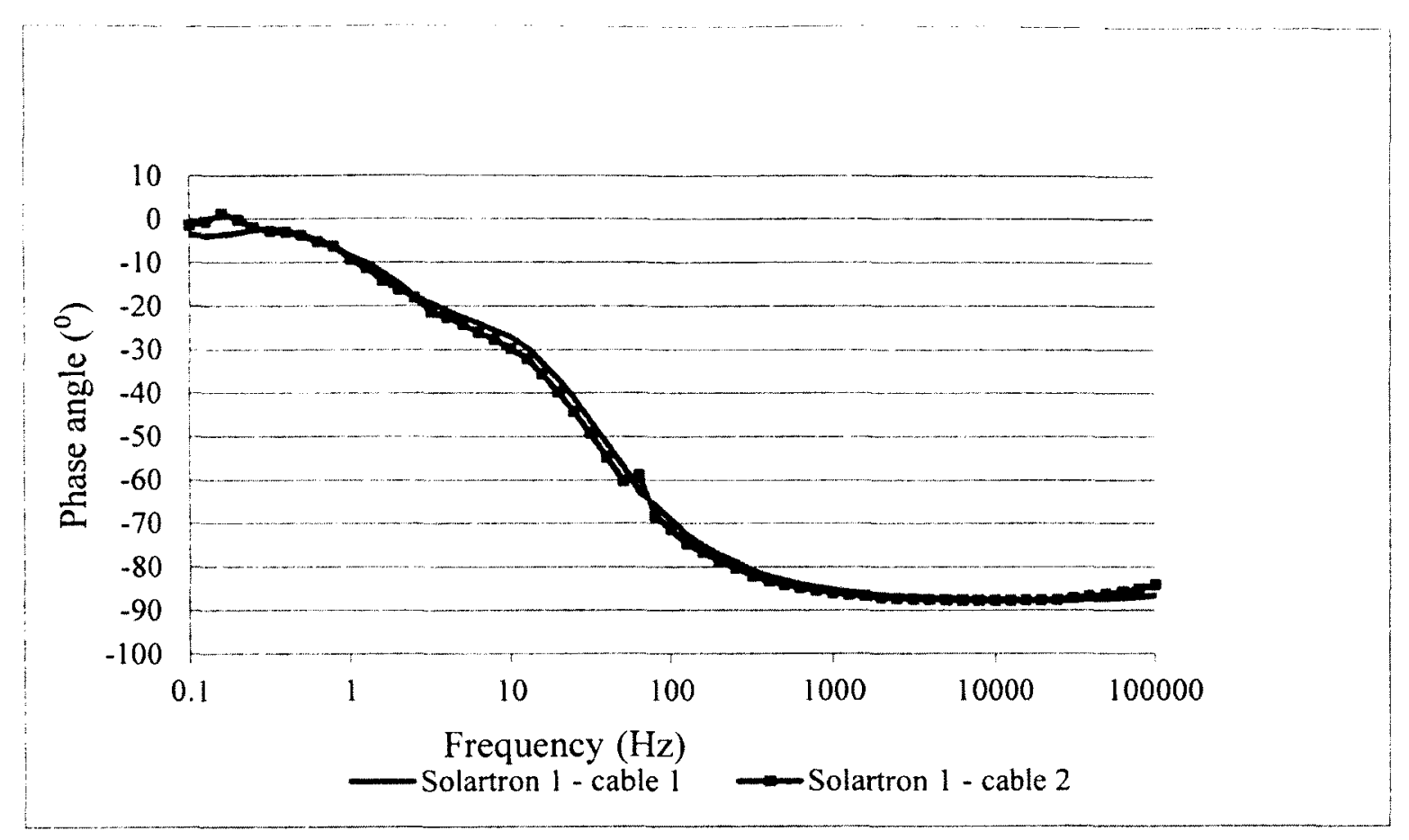

Figure 5-2 Phase angle measurements (HC, $1.0 \pm 0.1 \mathrm{~cm}^{2}$ ) for coated aluminum using Solartron 1 with different cables.

A high-impedance polymer sample was placed in the EIS cell with tap water as the electrolyte. This is an ultra-stable system, which always gives highly reproducible results. This time, differences in the phase-angle EIS spectra using the Gamry instruments with different cables were very noticeable and reproducible (Figure 5-3 to Figure 5-6). 


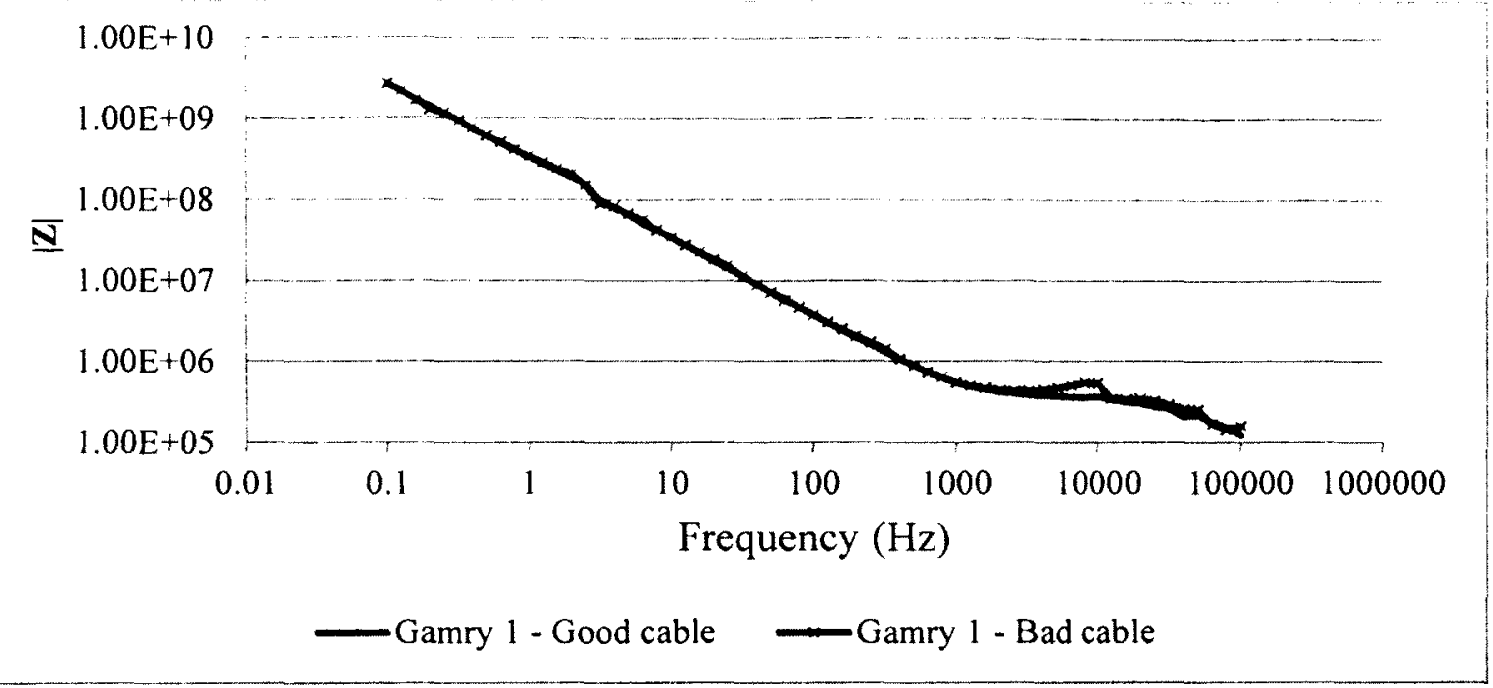

Figure 5-3 Impedance $(\Omega)$ measurements (HC, $1.0 \pm 0.1 \mathrm{~cm}^{2}$ ) for polymer using Gamry 1 with good and bad cables.

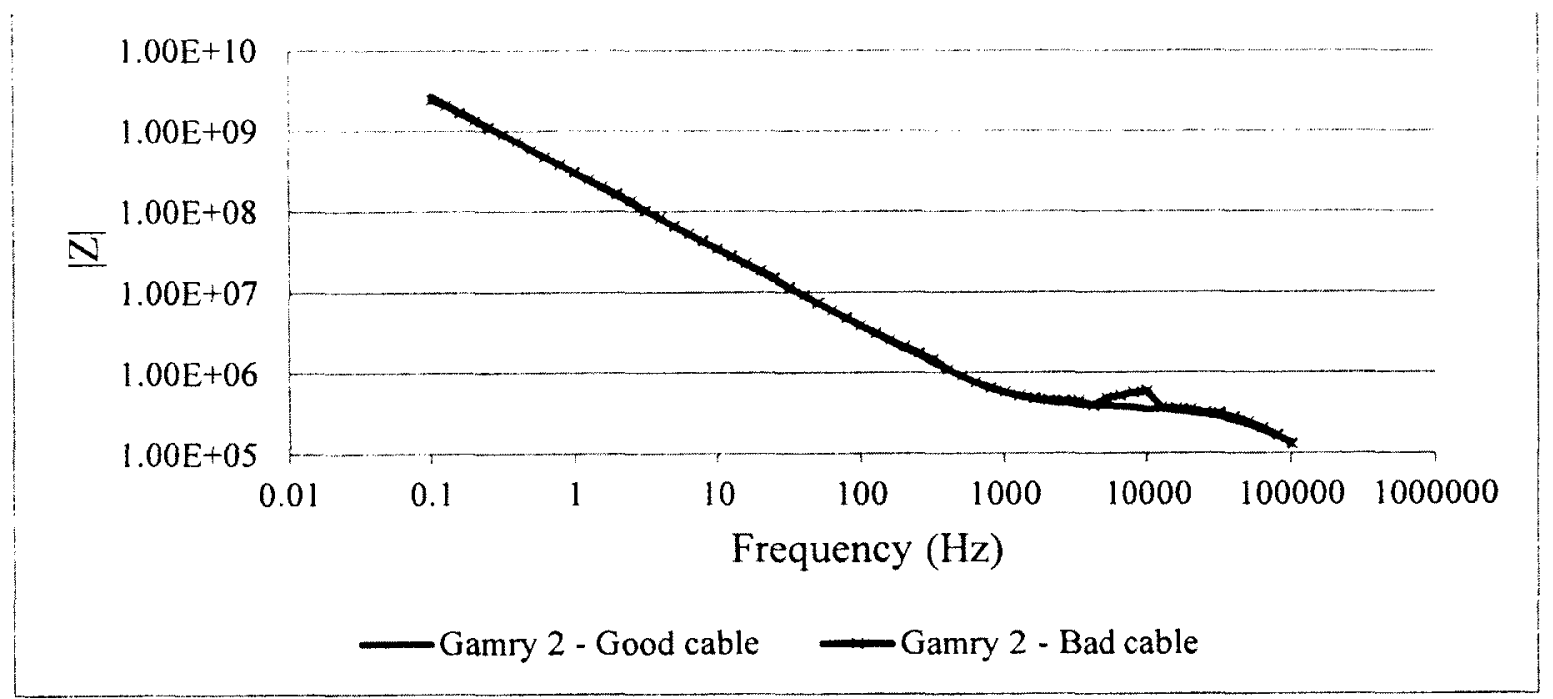

Figure 5-4 Impedance $(\Omega)$ measurements $\left(\mathrm{HC}, 1.0 \pm 0.1 \mathrm{~cm}^{2}\right)$ for polymer using Gamry 2 with good and bad cables.

Looking at Figure 5-3 and Figure 5-4, there is a small observable difference between the impedance measurements when using a bad cable as opposed to a good cable, but this is not really significant and might be ignored. There is a small jump in the impedance at about $10 \mathrm{kHz}$, occurring on both Gamry 1 and Gamry 2 when a bad cable is 
used. The differences are much more apparent and readily observable in the phase angle plots (Figure 5-5 and Figure 5-6).

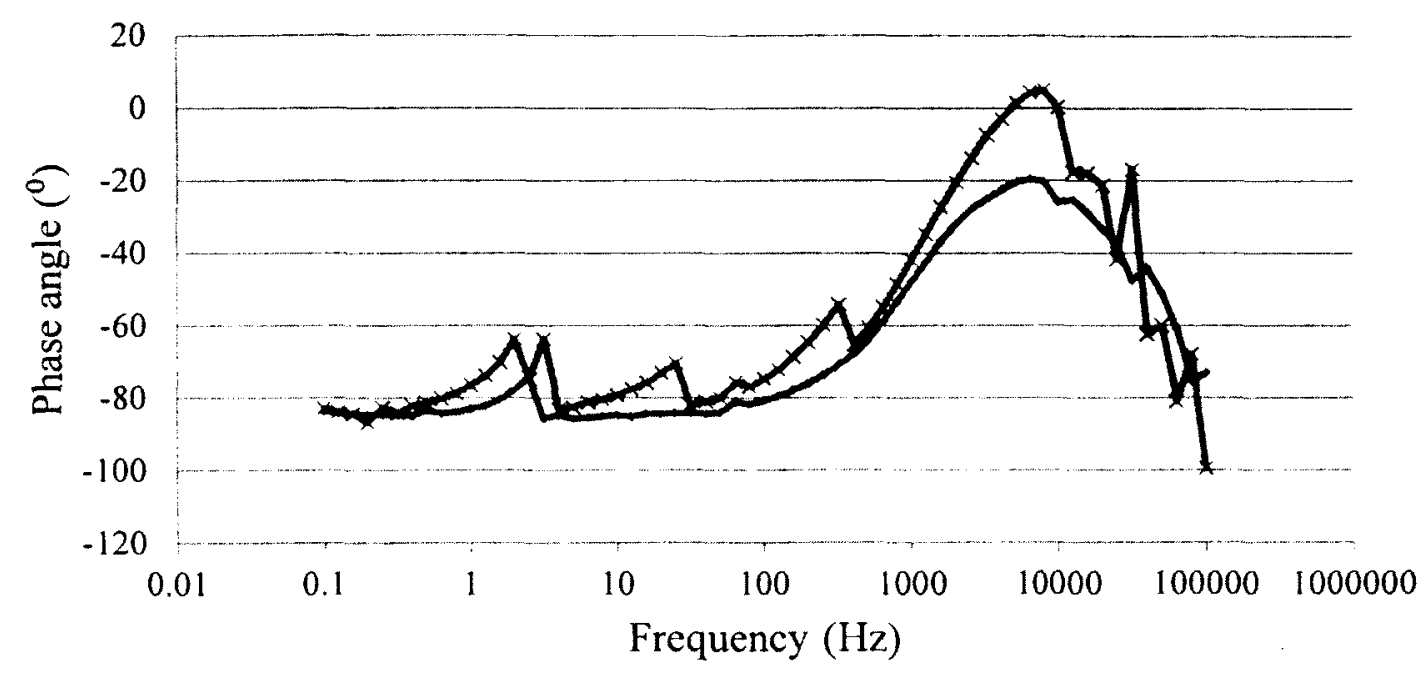

- Gamry 1 - Good cable - Gamry 1 - Bad cable

Figure 5-5 Phase angle measurements $\left(\mathrm{HC}, 1.0 \pm 0.1 \mathrm{~cm}^{2}\right.$ ) for polymer using Gamry 1 with good and bad cables.

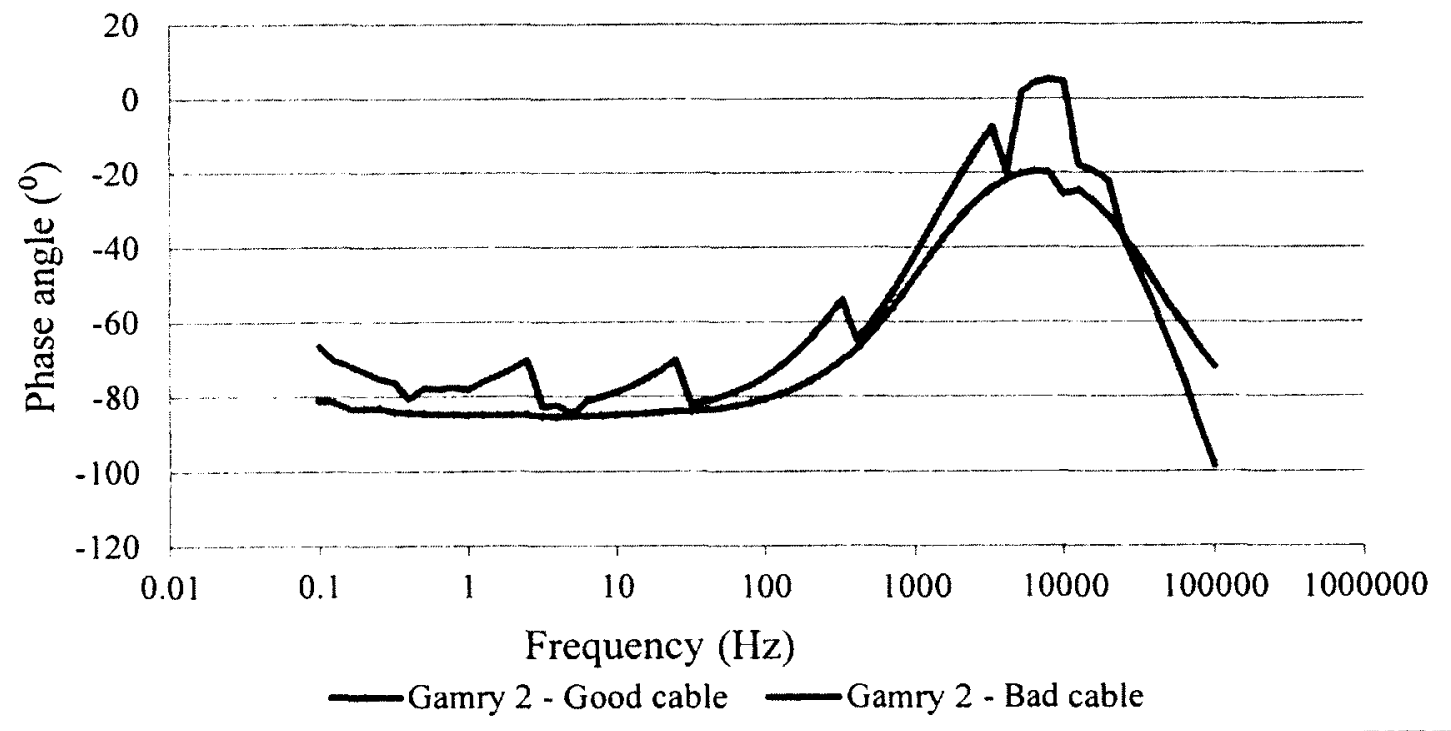

Figure 5-6 Phase angle measurements $\left(\mathrm{HC}, 1.0 \pm 0.1 \mathrm{~cm}^{2}\right)$ for polymer using Gamry 2 with good and bad cables. 
It can be seen from Figure 5-5 and Figure 5-6 that there is a difference in the phase-angle portion of the EIS spectra when a bad cable is used. With decreasing frequency, at $10 \mathrm{kHz}$ there is a step change in the phase angle measurements, suddenly becoming more positive, as was the case in the impedance plot. It can also be noted that the phase angle plot is no longer smooth and is "contaminated" with "phantom" spikes, which occur every decade of frequency.

These spikes are glitches, which appear to be a property of Gamry instruments. They arise every time the gain of the current amplifier changes when studying very high impedances. The phantom spikes are amplified by our "bad" cable, and are very apparent in the phase angle plots for both Gamry 1 and Gamry 2. The use of "good" cables reduces the magnitude of the phase-angle glitches and provides a much smoother curve. In this regard, Gamry 2 appears to perform better than Gamry 1 . Once the cable problem was identified, in all experiments other than those discussed in this section, good cables were utilized for the collection of EIS data.

\subsubsection{Electrical Noise Interference and Instrument Limits}

In order to assess electrical noise interference, several experiments were performed, several times, at two different locations (two different buildings). Some experiments were also performed inside a Faraday cage and repeated outside the cage for comparison (discussed in section 5.2.9). A Faraday cage is an electrically grounded metal box that prevents external electric and electromagnetic fields from interfering with measurements performed inside the box.

The open-lead, open-circuit spectrum test is a simple test to assess the highimpedance capabilities of an EIS instrument. An EIS scan is performed with no cell 
connected to the leads. These "open-lead" spectra demonstrate the highest impedance that the system can measure.

In Figure 5-7, the open-lead spectra for Solartron 1 (at 2 locations) and Solartron 2 were measured. All the variables such as applied voltage and integration methods were kept the same for measuring EIS spectra, so as to give a meaningful comparison.

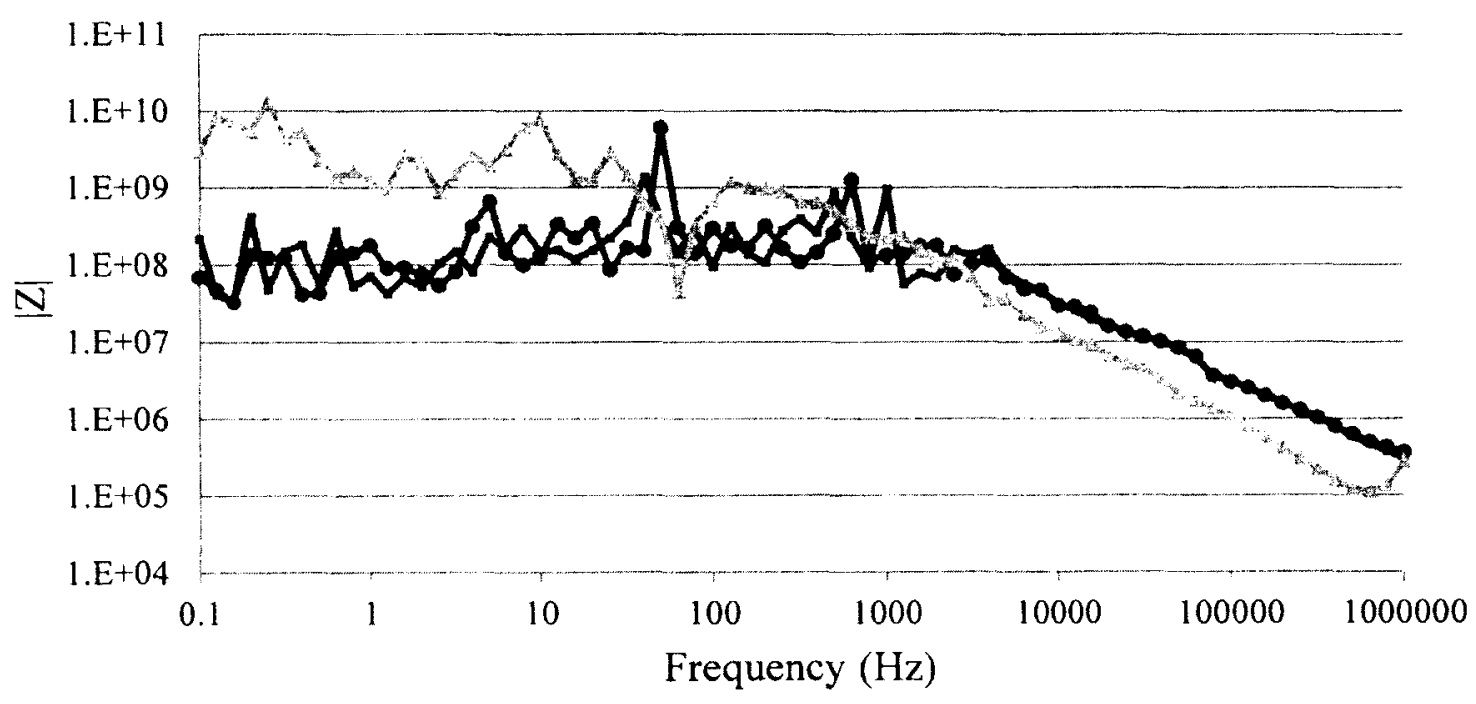

-Solartron 1 Location $1-$ Solartron 1 Location $2 \quad \cdots$ Solartron 2 Location 1

Figure 5-7 Open-lead spectra for Solartron 1 and Solartron 2.

From Figure 5-7, a lot of information can be drawn. There is no obvious difference between the spectra obtained at location 1 as compared to location 2; no significant extraneous electrical noise is demonstrated in the spectra. Concerning the system limits, it can be seen that Solartron 1 has an impedance measurement cap of approximately $100 \mathrm{M} \Omega$ starting at $1 \mathrm{kHz}$ and down to $100 \mathrm{mHz}$. In comparison, Solartron 2 has a higher impedance cap between $1 \mathrm{G} \Omega-10 \mathrm{G} \Omega$, subject to noise below $200 \mathrm{~Hz}$.

The open-lead spectra for Gamry 1 and Gamry 2 are shown in Figure 5-8. It can be seen that the performances of Gamry 1 and Gamry 2, as expected, are almost identical. 
They display much less noise than the Solartron instruments, and they have a higher impedance cap, $>1 \mathrm{~T} \Omega$ at $100 \mathrm{mHz}$. In order to once again compare location 1 and location 2, the open-lead spectra for Gamry 1 were recorded at both locations for comparison, Figure 5-9.

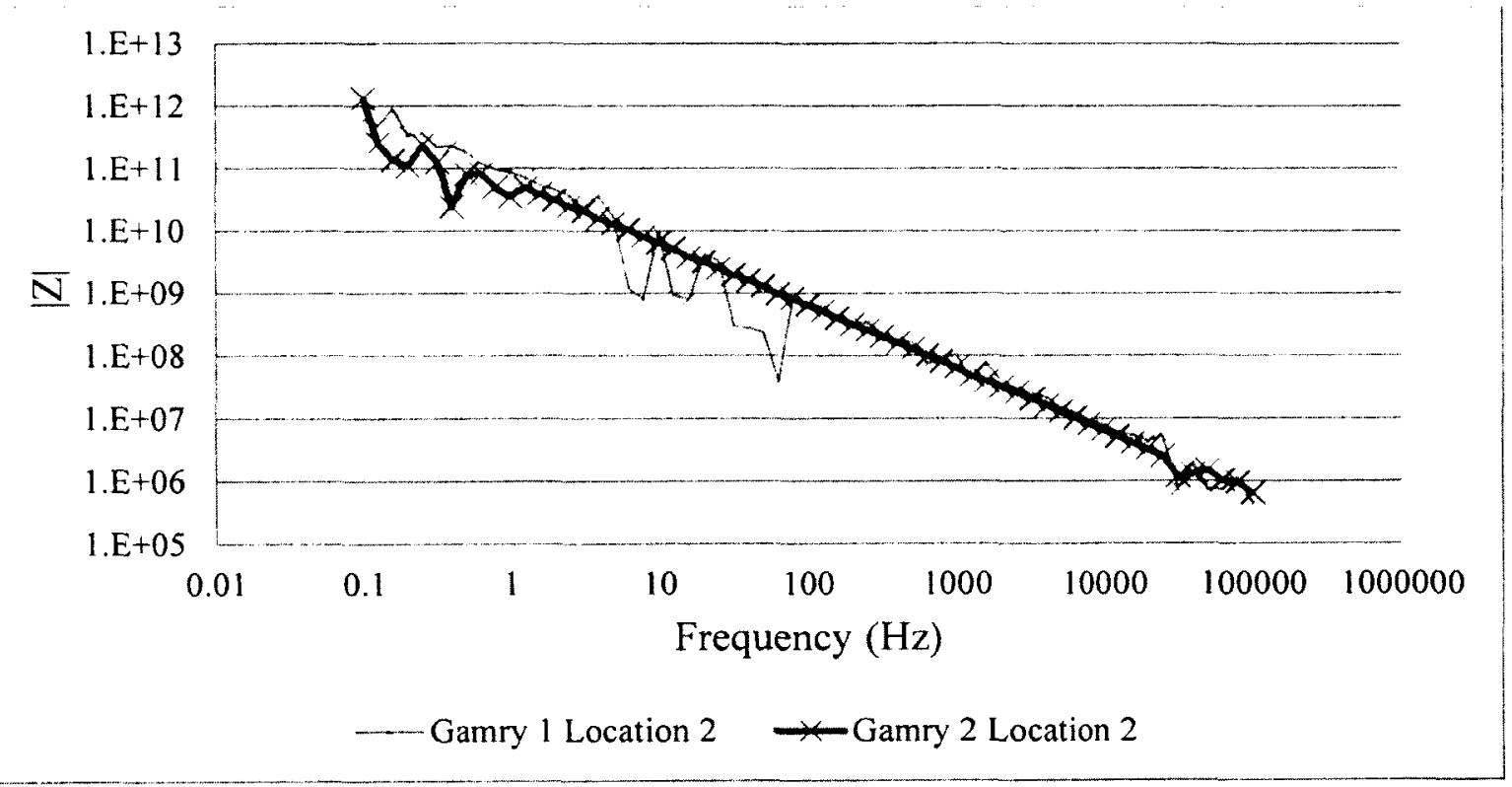

Figure 5-8 Open-lead spectra for Gamry 1 and Gamry 2.

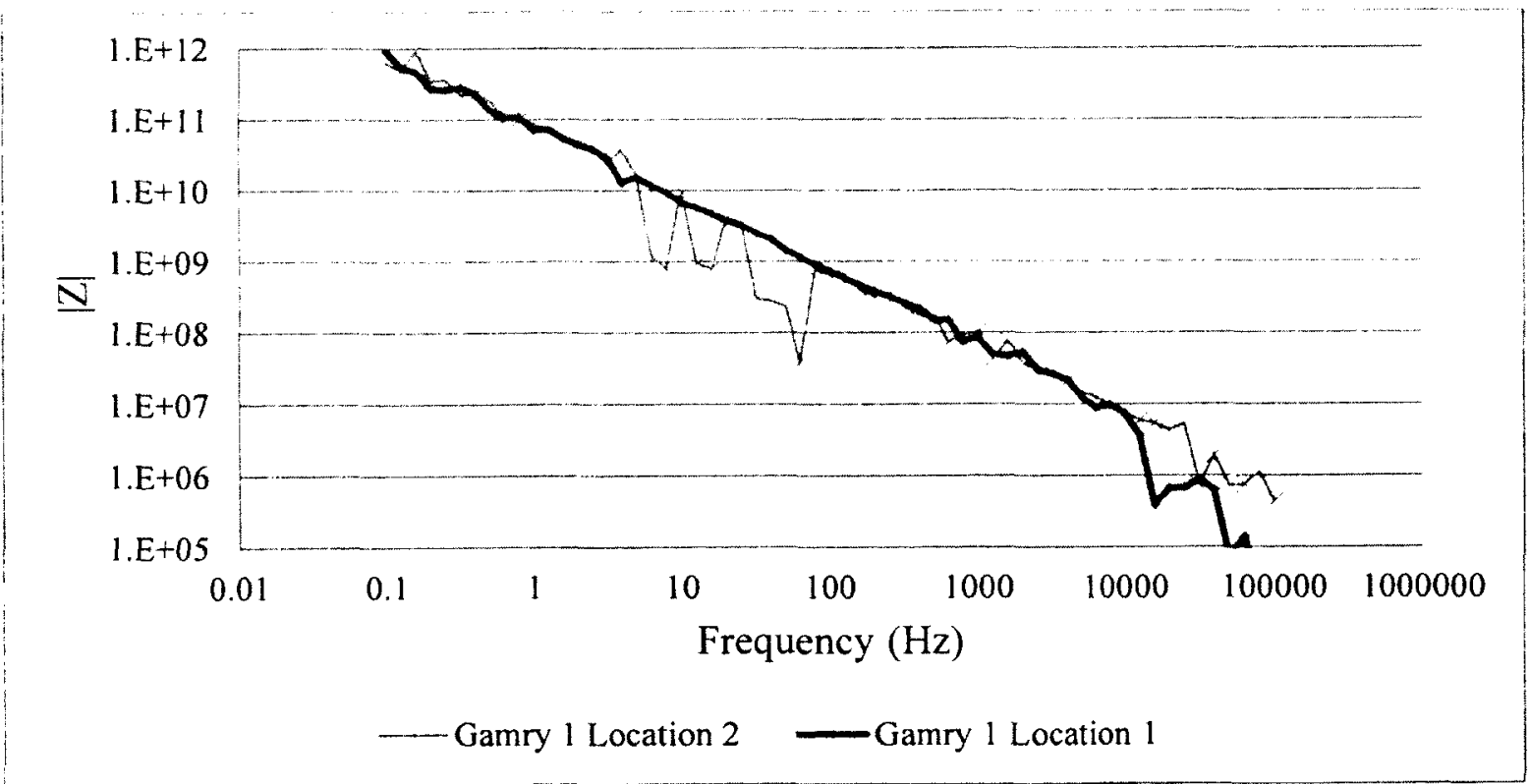

Figure 5-9 Open-lead spectra for Gamry 1 at location 1 and location 2. 
It can be seen from Figure 5-9 that for the most part both plots are almost identical, and demonstrate consistency in the performance of this instrument. The figure also shows that there is more noise picked up in location 2 than location 1 , above $1 \mathrm{G} \Omega$ $(<100 \mathrm{~Hz})$. All experiments henceforth were performed in location 1.

\subsubsection{Polymer Experiments}

Before recording the EIS spectra for coated-aluminum samples, a chemically inert, very

high-impedance polymer was studied and EIS data were collected.

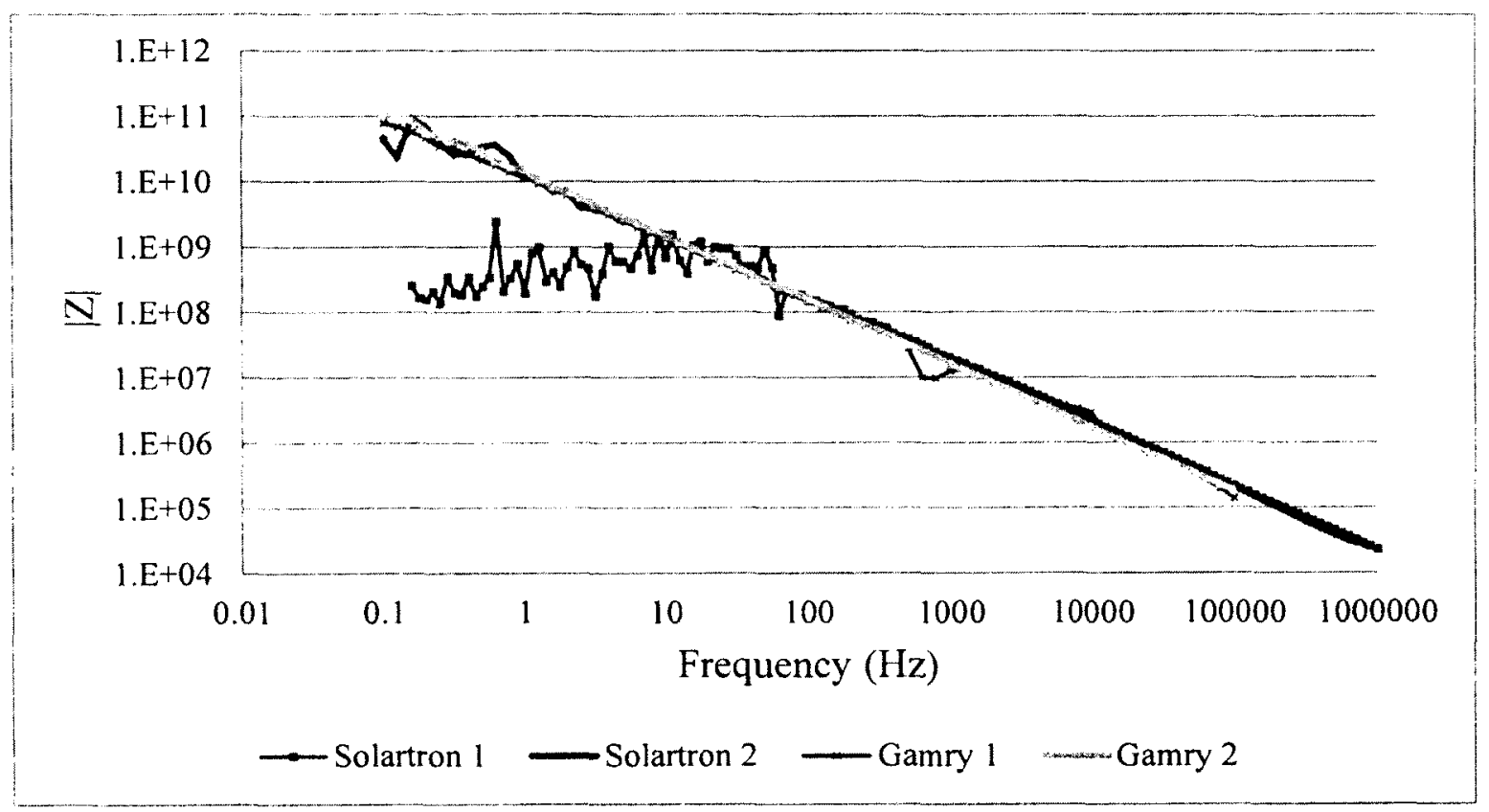

Figure 5-10 Impedance $(\Omega)$ measurements $\left(\mathrm{HC}, 1.0 \pm 0.1 \mathrm{~cm}^{2}\right)$ for polymer in water using Solartron 1, Solartron 2, Gamry 1 and Gamry 2.

Looking at Figure 5-10, it can be seen that the low-frequency impedance of the polymer is in excess of $100 \mathrm{G} \Omega$, far surpassing the measuring capabilities of Solartron 1, but lying within the measuring range for the other instruments. Notably, Solartron 2 performs better in this experiment than in the open-lead experiment (compare Figure 5-10 
and Figure 5-7), but appears to be at its limit. It displays much less noise than in Figure 5-7.

The Gamry instruments record lower high-frequency impedances for the polymer than for their open-lead measurements, implying that this is a true measurement and not a limit. This experiment demonstrated the consistency of the results between all the machines. Even though for this specific sample, Solartron 1 fails to provide good data around $1 \mathrm{G} \Omega$ and below $100 \mathrm{~Hz}$, it is still suitable for studying samples with impedances below $1 \mathrm{G} \Omega$ (see Figure 5-1 and Figure 5-2).

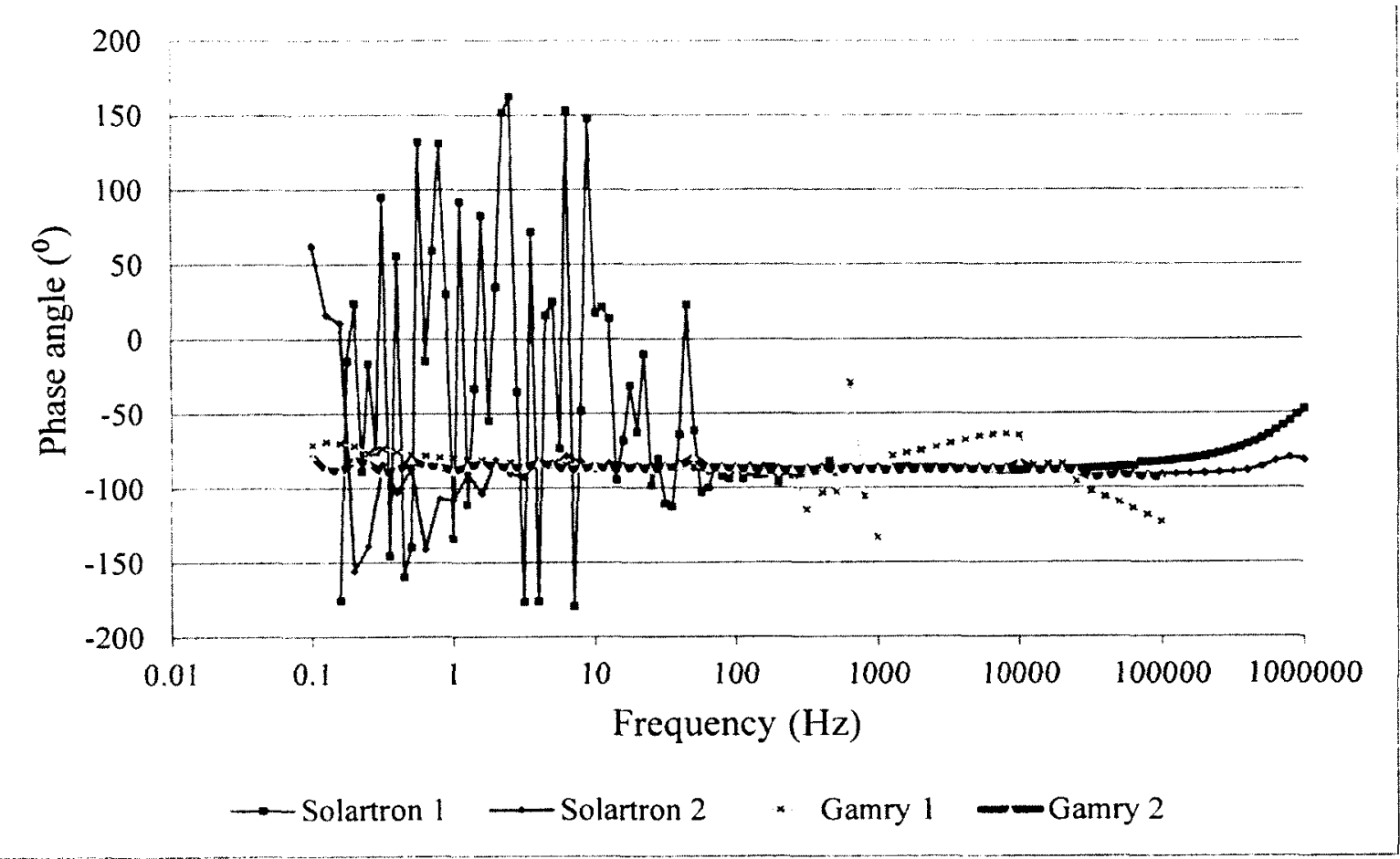

Figure 5-11 Phase angle measurements $\left(\mathrm{HC}, 1.0 \pm 0.1 \mathrm{~cm}^{2}\right.$ ) for polymer in water using Solartron 1, Solartron 2, Gamry 1 and Gamry 2.

On a first look at Figure 5-11, it can be seen that all the curves are consistent in giving a phase angle measurement of approximately $-90^{\circ}$ across the complete frequency spectrum, as expected for a high impedance polymer. It can also be seen that Solartron 1 
produces an exceptionally noisy phase angle plot below $100 \mathrm{~Hz}$, with an average value of $-90^{\circ}$. At first glance, this unstable spectrum might be attributed to electrical noise picked up by the instrument, but no such noise was picked up by the other instruments. After careful analysis of impedance and phase angle data, it appears that this spiking in the phase angle is due to the machine being pushed past its limits at an impedance of $1 \mathrm{G} \Omega$. On the other hand, Solartron 2 is not being pushed to its limit until impedances $\geq 10 \mathrm{G} \Omega$ (see Figure 5-10). Such impedance, $\geq 10 \mathrm{G} \Omega$, only occurs at frequencies $<10 \mathrm{~Hz}$, and as seen in Figure 5-11, the phase-angle plot for Solartron 2 starts to produce noise spikes below $10 \mathrm{~Hz}$.

\subsubsection{Fuel Cell Experiments}

After ensuring that all systems are comparable and consistent when collecting EIS data for high impedance coatings, experiments at the lower end of the impedance spectrum were performed to ensure consistency at all magnitudes of impedance and to investigate the phenomenon of mutual inductance. As an example of a low-impedance circuit, EIS spectra for a direct methanol fuel cell were collected using Gamry 1, Gamry 2 and Solartron $1[40]$. 


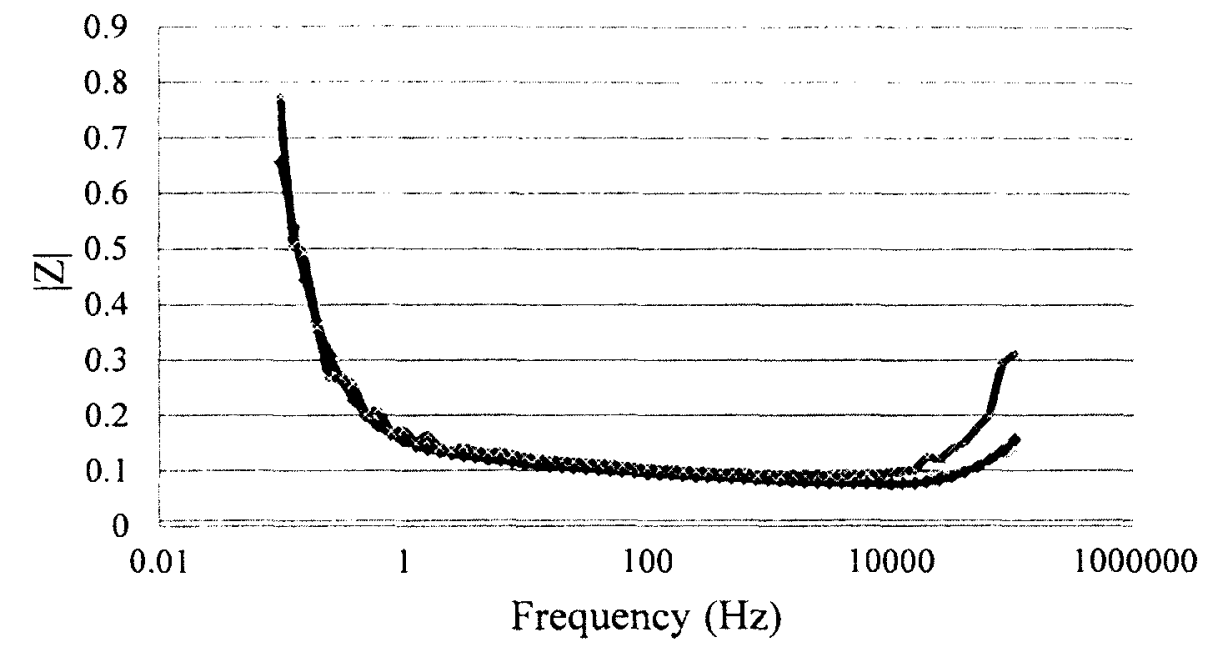

-.- Gamry 1 - Gamry 2 - Solartron 1

Figure 5-12 Impedance ( $\Omega$ ) measurements for fuel cell.

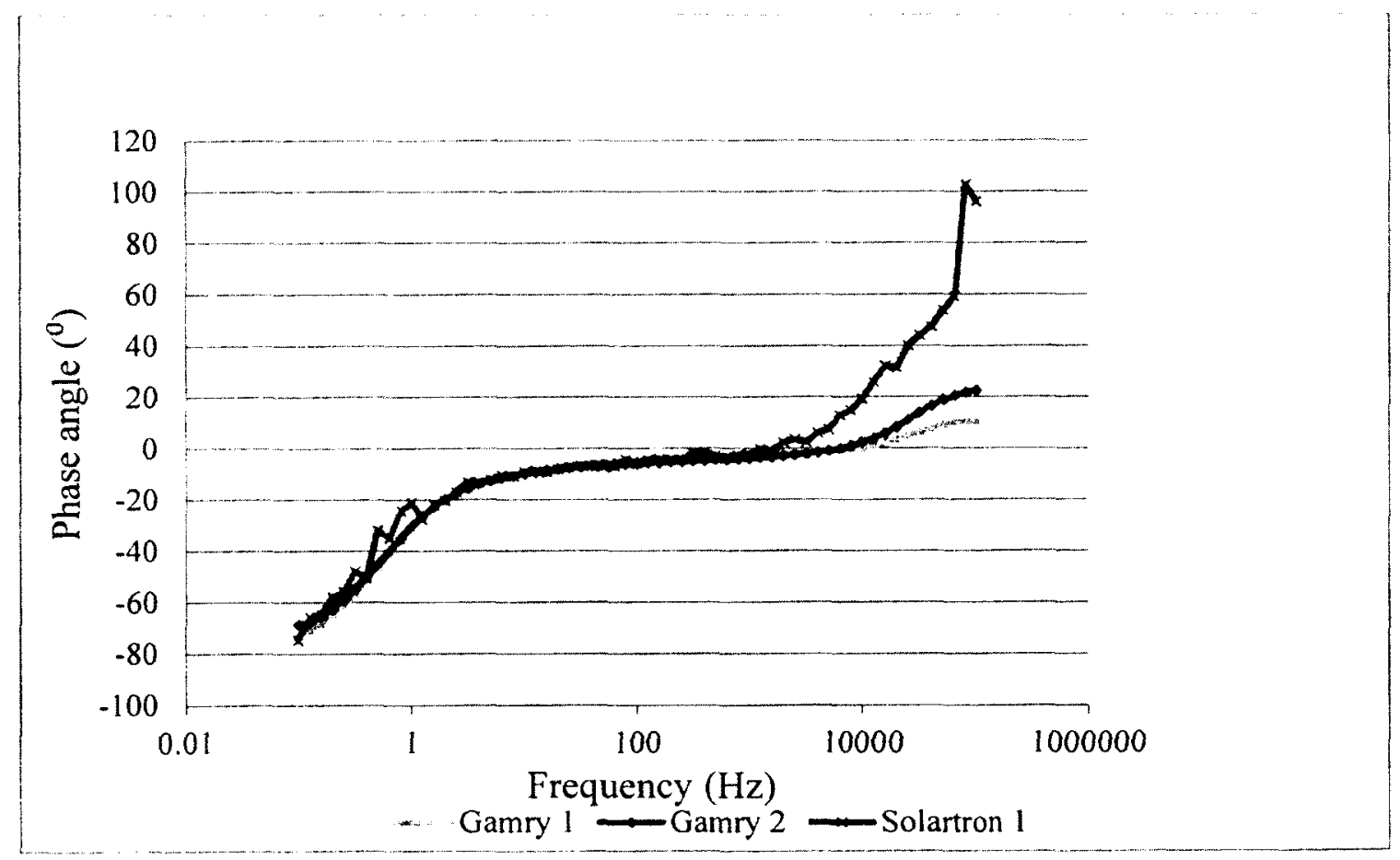

Figure 5-13 Phase angle measurements for fuel cell. 
From Figure 5-12 and Figure 5-13, it can be seen that the impedance measurements and phase angle measurements are almost identical over much of the spectra for all three systems. At the high end of the frequency spectrum, the mutual inductance effect can be observed. Solartron 1 shows a higher inductance as compared to Gamry 1 and Gamry 2. The performance of Solartron 1 becomes questionable for low impedance systems at frequencies above $10 \mathrm{kHz}$.

Overall it was confirmed that over most of the EIS spectrum (100 mHz $-40 \mathrm{kHz})$, the potentiostats have similar performances and provide consistent results for highimpedance and low-impedance systems.

\subsubsection{Test Circuits}

After checking the consistency of the potentiostats at very high impedances and very low impedances, it was important to check the consistency in mid-range. It is the custom of the manufacturers of EIS equipment to provide standard test circuits to check the quantitative accuracy of their instruments. Figure 5-14 shows such a circuit, consisting of a combination of resistors and capacitors in series and parallel.

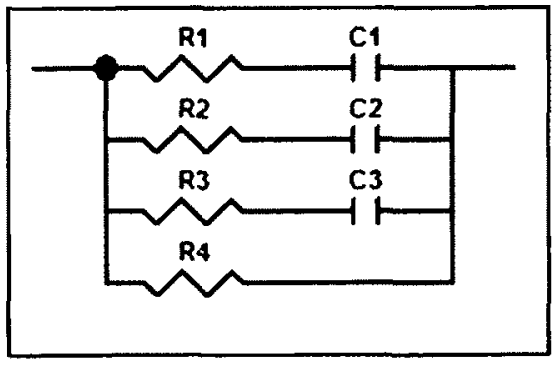

Figure 5-14 Components of test circuit. 
Table 5-1 provides the manufacturer's values of the different components, and includes their values derived from fits to the EIS data $^{2}$, along with their percentages of error, for the 4 different potentiostats. The results are very satisfactory.

\section{Table 5-1 Component values and their percentages of error ${ }^{3}$, as fitted to the circuit} data generated by the 4 different potentiostats.

\begin{tabular}{|c|c|c|c|c|c|c|c|c|c|}
\hline \multirow{2}{*}{$\begin{array}{l}\text { Circuit } \\
\text { element }\end{array}$} & \multirow{2}{*}{$\begin{array}{l}\text { OEM } \\
\text { value }^{4}\end{array}$} & \multicolumn{2}{|c|}{ Solartron 1} & \multicolumn{2}{|c|}{ Solartron 2} & \multicolumn{2}{|c|}{ Gamry 1} & \multicolumn{2}{|c|}{ Gamry 2} \\
\hline & & Value & $\%$ & Value & $\%$ & Value & $\%$ & Value & $\%$ \\
\hline RI & $1.54 \mathrm{E}+01$ & $1.58 \mathrm{E}+01$ & 1 & $1.57 \mathrm{E}+01$ & 1 & $1.59 E+01$ & 2 & $1.61 \mathrm{E}+01$ & 2 \\
\hline $\mathrm{R} 2$ & $3.01 E+04$ & $2.96 \mathrm{E}+04$ & 3 & $2.97 \mathrm{E}+04$ & 2 & $3.28 \mathrm{E}+04$ & 2 & $2.93 E+04$ & 2 \\
\hline R3 & $3.48 E+05$ & $3.46 \mathrm{E}+05$ & 8 & $3.44 \mathrm{E}+05$ & 3 & $3.63 E+05$ & 4 & $3.42 \mathrm{E}+05$ & 4 \\
\hline R4 & $1.00 \mathrm{E}+08$ & $1.06 \mathrm{E}+08$ & 7 & $9.49 \mathrm{E}+07$ & 5 & $9.48 \mathrm{E}+07$ & 6 & $9.64 \mathrm{E}+07$ & 6 \\
\hline $\mathrm{Cl}$ & $1.0 \mathrm{E}-07$ & $1.01 \mathrm{E}-07$ & 1 & $9.93 \mathrm{E}-08$ & 1 & $1.10 \mathrm{E}-07$ & 0 & $9.83 \mathrm{E}-08$ & 0 \\
\hline $\mathrm{C} 2$ & $2.2 \mathrm{E}-07$ & $2.29 \mathrm{E}-07$ & 3 & $2.29 \mathrm{E}-07$ & 2 & $2.27 \mathrm{E}-07$ & 2 & $2.28 \mathrm{E}-07$ & 2 \\
\hline $\mathrm{C} 3$ & $3.3 \mathrm{E}-07$ & $3.11 \mathrm{E}-07$ & 3 & $3.24 \mathrm{E}-07$ & 1 & $3.14 \mathrm{E}-07$ & 2 & $3.21 \mathrm{E}-07$ & 2 \\
\hline
\end{tabular}

In the following graphs, Figure 5-15 and Figure 5-16, the impedance and phaseangle measurements for the Solartron instruments and Gamry instruments again demonstrate their consistency in the measurement of the test circuit.

\footnotetext{
${ }^{2}$ ZPlot (for Solartron instruments) and EchemAnalyst (for Gamry instruments) were used to simulate the circuit in Figure 5-14 and estimate component values; see section 4.7.

${ }^{3}$ The Error estimates are calculated by ZPlot (for Solartron instruments) and EchemAnalyst (for Gamry instruments); see section 4.7

${ }^{4}$ OEM (original equipment manufacturer) errors were not available
} 


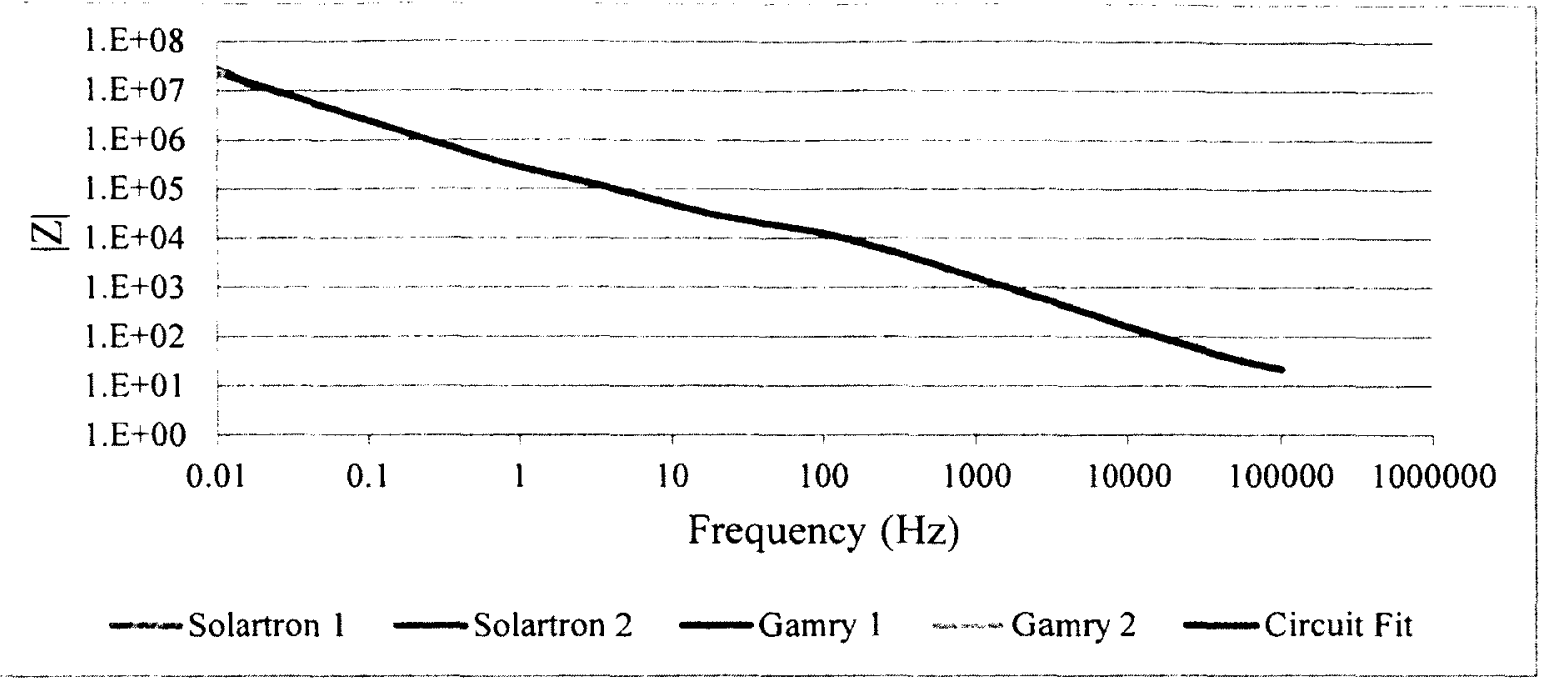

Figure 5-15 Impedance $(\Omega)$ measurements for test circuit.

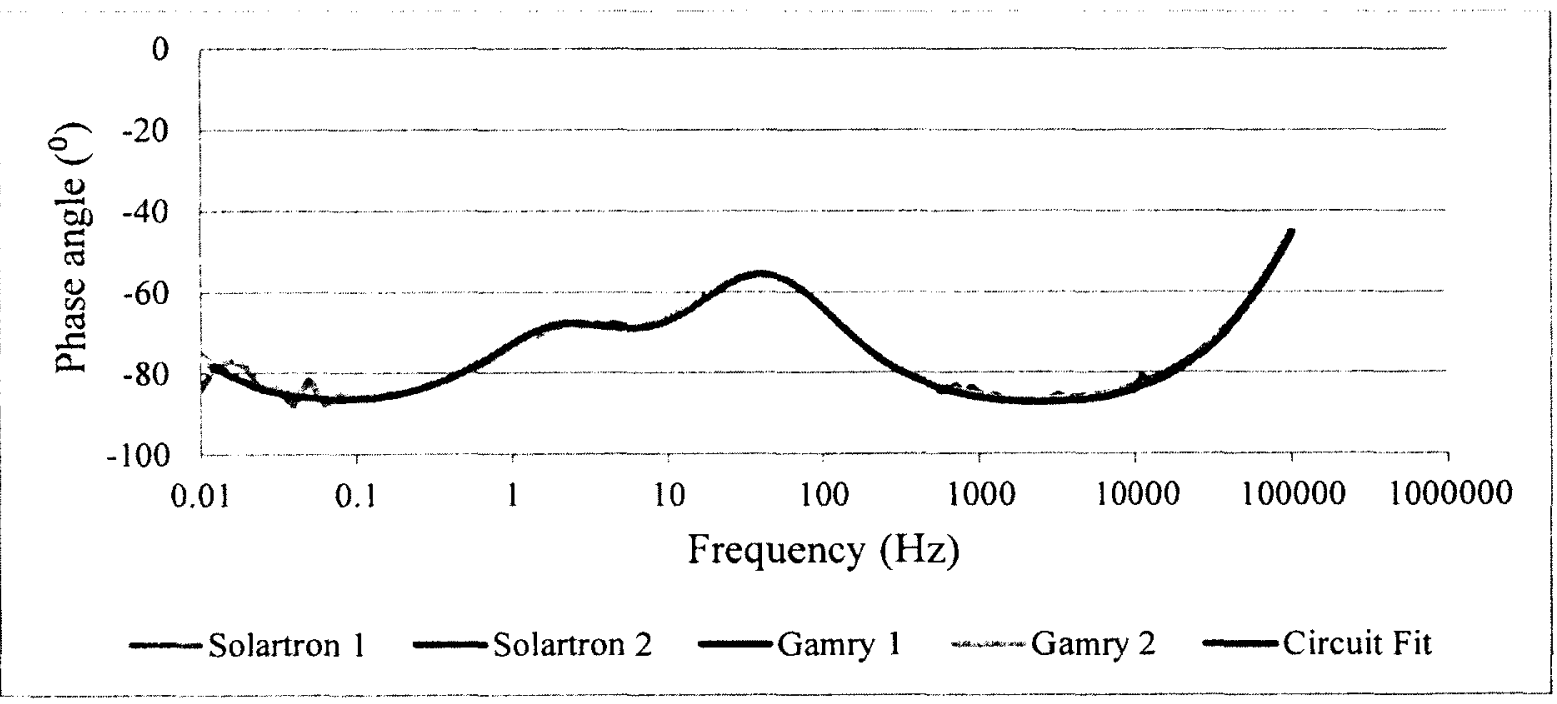

Figure 5-16 Phase angle measurements for test circuit.

\subsubsection{Other Tests on Solartron 1}

Since Solartron 1 was initially the primary machine used for EIS data collection, some additional basic tests were conducted to finalize the assessment of the instrument. EIS measurements were conducted on a $70 \Omega$ resistor, an $8 \mathrm{M} \Omega$ resistor and a $100 \mathrm{nF}$ capacitor (Figure 5-17 and Figure 5-18). 


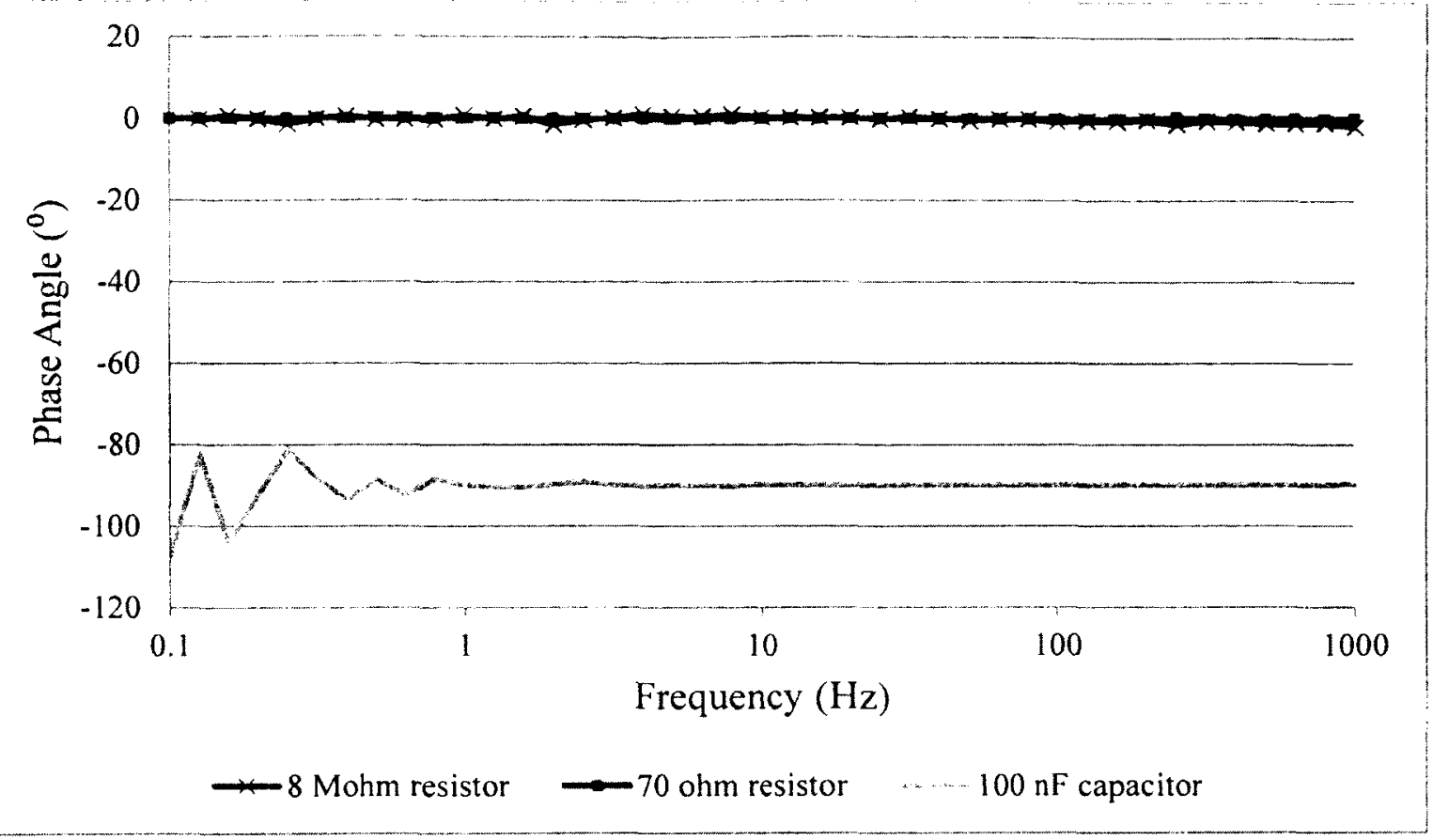

Figure 5-17 Phase Angle measurements for circuit elements using Solartron 1.

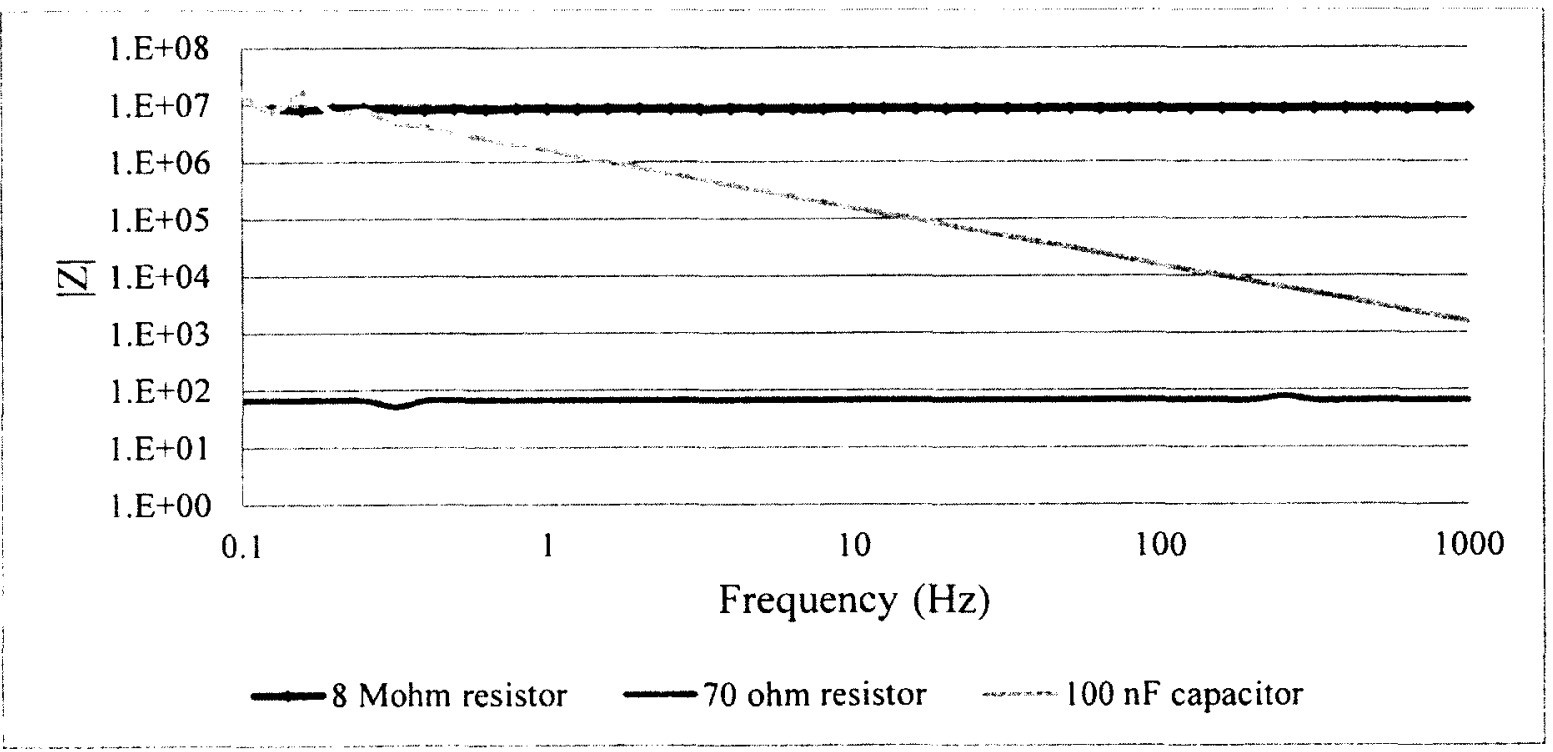

Figure 5-18 Impedance $(\Omega)$ measurements for circuit elements using Solartron 1.

The EIS output provided by Solartron 1 for the resistors and capacitors was correct. The resistors showed a phase angle of $0^{\circ}$ across the spectrum and the capacitor showed a phase angle of $-90^{\circ}$. In the impedance plots, the EIS measured the correct value 
of the resistor and showed a line with a slope of zero. A line with a slope of -1 was obtained, as expected, for the capacitor. When the capacitance of the capacitor was calculated using the Solartron circuit-fitting software, it provided a value for the capacitor in agreement with the manufacturers value, $103 \pm 2 \mathrm{nF}$. A little noise was observable as the capacitor's impedance approached $10 \mathrm{M} \Omega$.

\subsubsection{Coated Aluminum Samples}

After all the preliminary performance checks were done, the limits of operation of Solartron instruments and Gamry instruments had been identified. It was important to understand whether coated aluminum samples would fall within the operating range of the equipment or not, so EIS spectra were run using high quality, i.e. high-impedance, coated aluminum samples. The samples were placed in the vertical cell using water as the electrolyte, and tested in the manner used in previous experiments. 


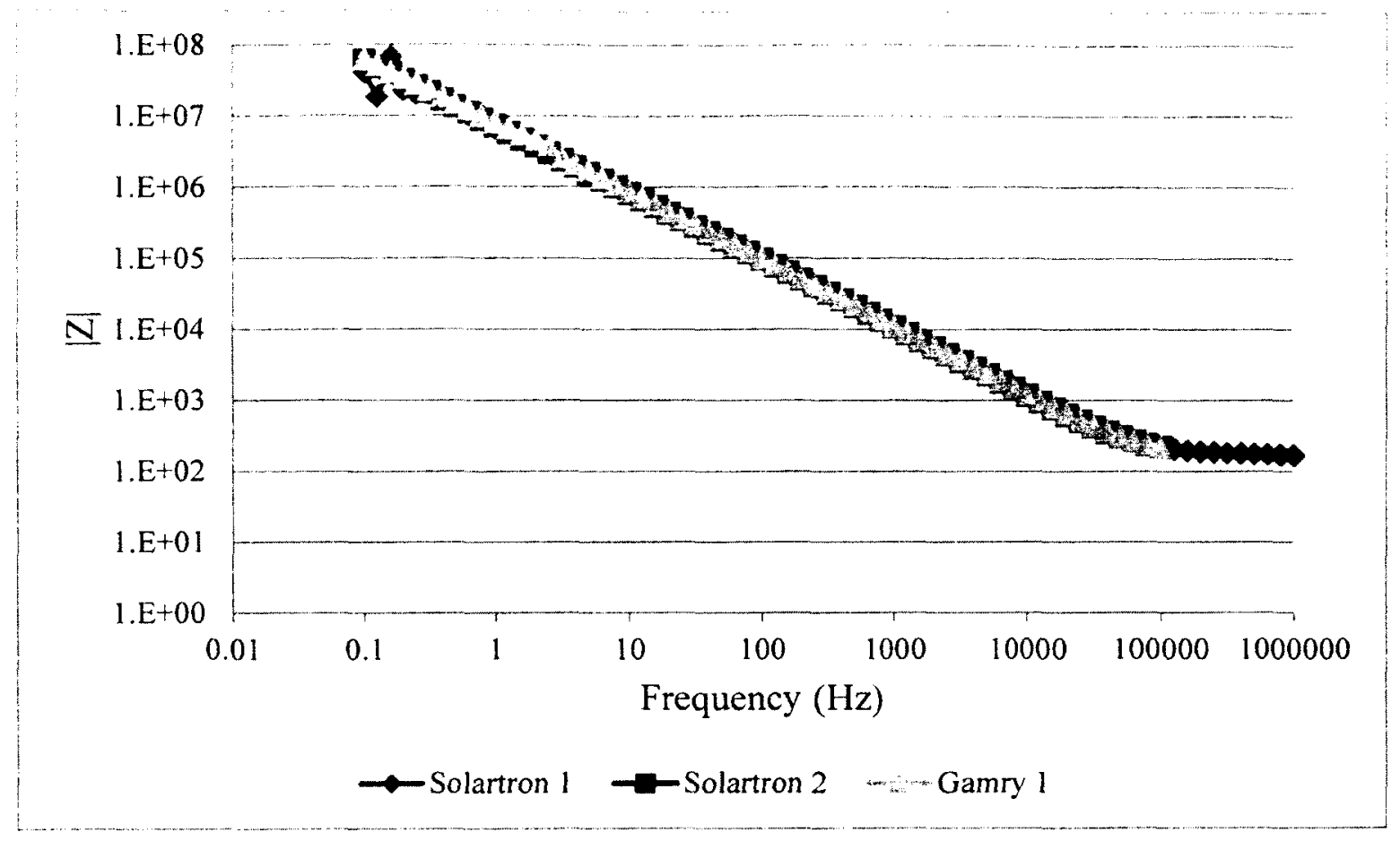

Figure 5-19 Impedance $(\Omega)$ measurements $\left(\mathrm{HC}, 1.0 \pm 0.1 \mathrm{~cm}^{2}\right)$ for intact coated aluminum sample.

Looking at Figure 5-19, the consistency of the potentiostats is once again confirmed. It is observed that the impedance of the coated aluminum samples is approximately $100 \mathrm{M} \Omega$ at $0.1 \mathrm{~Hz}$, which is within the operating range of Solartron 2, Gamry 1 and Gamry 2, but is close to the upper limit of impedance for Solartron 1. This means that all data collected at impedances below $100 \mathrm{M} \Omega$ will be accurately measured. In corrosive electrolytes, measured impedances for coated aluminum samples will be lower, and any changes in the EIS spectra that occur can be positively recorded as signatures of corrosion. 


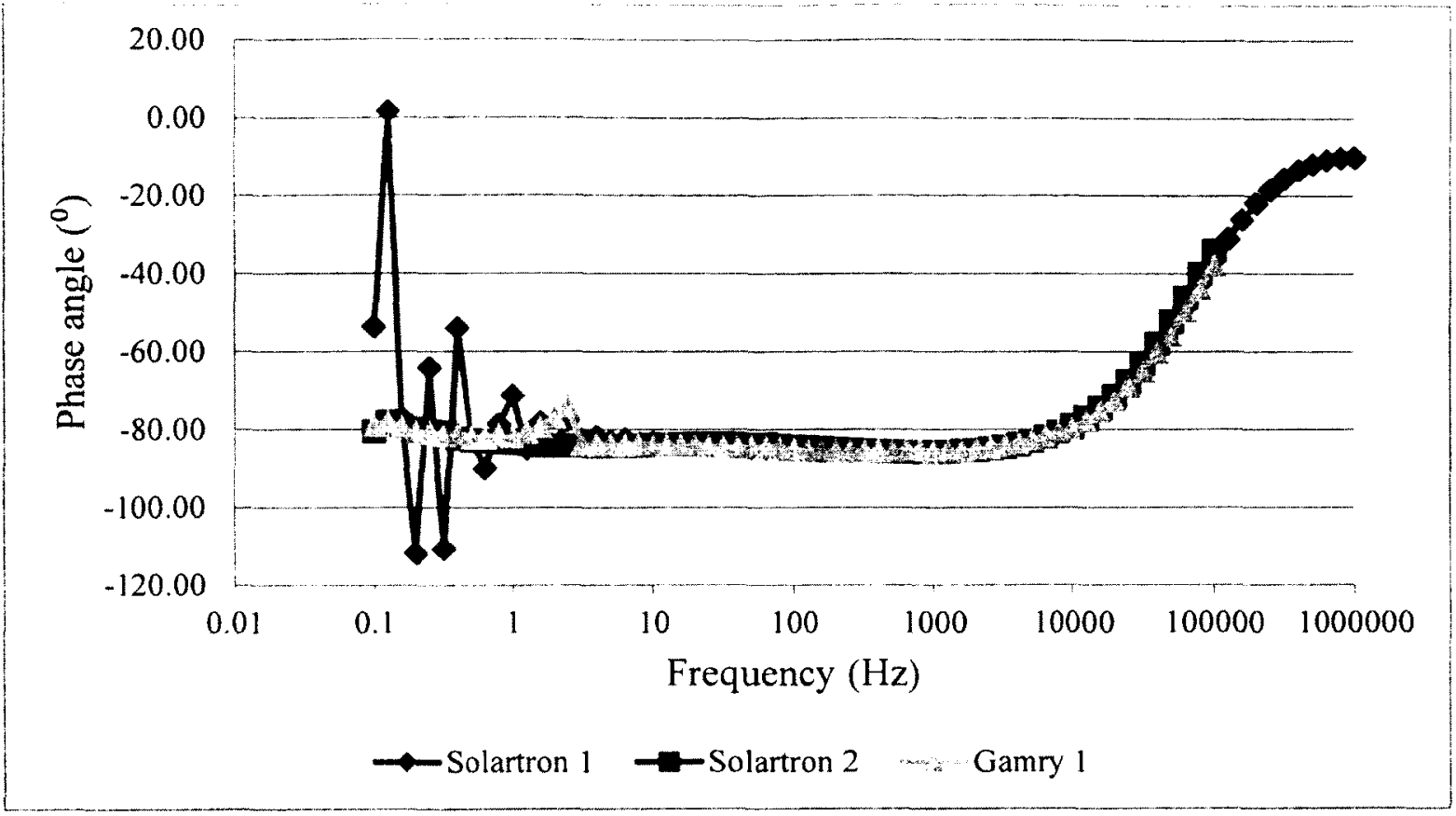

Figure 5-20 Phase angle measurements $\left(\mathrm{HC}, 1.0 \pm 0.1 \mathrm{~cm}^{2}\right)$ for intact coated
aluminum sample. For the most part, the phase-angle measurements for the different instruments are consistent, Figure 5-20. All the systems provide a phase-angle measurement between $-80^{\circ}$ and $-90^{\circ}$ over most of the spectrum. Note that the phase angle is not exactly $-90^{\circ}$ as observed for the capacitor and the polymer. The coated aluminum is not a pure capacitor.

Significant spikes are observed in the phase-angle data of Solartron 1, at frequencies dropping below $1 \mathrm{~Hz}$, which again is symptomatic of the fact that this instrument at these impedances and frequencies is close to its limits. As will be seen, once aluminum samples start to corrode, their impedance drops and this machine can operate comfortably within its limits. 


\subsubsection{Faraday Cell Experiments}

When the EIS spectra performed at location 1 were compared to the data from location 2, there was little difference in the results (Figures 5-7 to 5-9). Extraneous noise did not significantly affect the data. This was confirmed with the use of a Faraday cage, in which the electrochemical cell was placed. EIS results with and without a Faraday cage were identical. This showed that the potentiostats were not susceptible to external noise, or that there was no significant external noise present in either location. Hence, either location is suitable for performing EIS experiments. On the occasions that significant noise was observed, instruments were operating close to or beyond their capabilities at either end of the frequency spectrum.

\subsubsection{Results}

- Only within certain operational limits, all potentiostats are consistent and provide essentially identical results;

- Gamry 1 and Gamry 2 have an impedance cap of approximately $1 T \Omega$ at $100 \mathrm{mHz}$

- Solartron 2 has an impedance cap of approximately $10 \mathrm{G} \Omega$ at $100 \mathrm{mHz}$;

- Solartron 1 has an impedance cap lower than $100 \mathrm{M} \Omega$ at $100 \mathrm{mHz}$;

- The polymer samples have an impedance of approximately $100 \mathrm{G} \Omega$ at $100 \mathrm{mHz}$;

- The best aluminum samples have an impedance of approximately $100 \mathrm{M} \Omega$ at $100 \mathrm{mHz}$ when the vertical cell is used; 
- Faulty cables in the Gamry systems can amplify phase glitches, caused by switching ranges in the current-gain amplifiers, and provide misleading spikes and measurements in the phase-angle plots;

- Under certain conditions, the potentiostats can exhibit noise, particularly in the phase-angle measurements. In this respect, Gamry 2 was the best performer, Gamry 1 the next best, and Solartron 2 was superior to Solartron 1;

- Differences between the levels of electrical noise observed in two different buildings were less than the differences between different instruments, and much less than effects produced by faulty cables;

- When collecting EIS data using the Solartron instruments, for low-impedance systems at high frequencies, $>10 \mathrm{kHz}$, there were signs of interference from inductance in cables.

\subsubsection{Conclusions}

Looking at all the results, it can be concluded that all the instruments have varying operational limits, within which they all work well and provide consistent and reproducible results.

For the Gamry systems, it is very important to ensure that the cables used for the EIS measurements do not produce phantom peaks in the phase angle spectra. These peaks arise when the current-gain amplifier changes its range, and may be associated with inductance in the leads, because this sudden current change produces a marked positive spike in the phase angle.

By having very high-impedance measurement capabilities, Gamry 1 and Gamry 2 are very well suited to measure EIS spectra for all kinds of samples over a wide range of 
frequencies. Solartron 2 is also capable of providing reliable results for our highest impedance studies of polymers, although these are close to its limit. On the other hand, Solartron 1 is not able to perform EIS experiments for the polymer samples at frequencies below $100 \mathrm{~Hz}$. It can start to have problems at impedances of $\sim 100 \mathrm{M} \Omega$.

From the low impedance fuel cell experiments, it was determined once again that all the systems were reliable and provided essentially the same results. In such lowimpedance measurements, any of the systems are suitable to run EIS spectra, although care must be taken because of inductive effects from cables that can appear at high frequencies when using the Solartron instruments.

Coated aluminum samples have their highest impedance of approximately $100 \mathrm{M} \Omega$ at $100 \mathrm{mHz}$, when using the vertical cell, but their impedances are generally lower than this. All the systems are capable of providing reliable EIS data for such samples. Thus, changes observed to occur in the EIS spectra of coated aluminum samples as corrosion occurs, are real and can be quantitatively measured.

\subsection{Aluminum Foil Experiments}

\subsubsection{Introduction}

It was not known how the coated aluminum samples would react once they were exposed to corrosive solutions. The previous section demonstrated a baseline for a chemically inert impervious system, where no reactions were taking place in the corrosion cells. As a baseline for a chemically active system, a number of experiments were run using the shiny side of kitchen aluminum foil using Solartron 1. It was expected that corrosion 
would occur in these experiments, and signatures for corrosion would be recorded and later compared with the corroding coated-aluminum samples.

\subsubsection{Al Foil Exposed to Tap Water}

As a starting point, tap water was used as the electrolyte to give a baseline for aluminum foil impedance data. Figure 5-21 shows the impedance and phase angle measurements for an aluminum foil sample in tap water. At the high frequency range $\left(10^{5} \mathrm{~Hz}-10 \mathrm{~Hz}\right)$, the impedance plot is a straight line with a slope of 0 , a resistor, indicating the solution resistance, with no coating resistance. Below $10 \mathrm{~Hz}$, the impedance plot becomes more like a capacitor. The capacitance is assigned to that of the metal/oxide/electrolyte interface on the surface. At the low end of the frequency spectrum, $\sim 0.2 \mathrm{~Hz}$, a transition from capacitive to resistive behaviour is observed. This is thought to be the resistance of the oxide on the metal.

The limiting impedance of $10 \mathrm{k} \Omega$ at $0.1 \mathrm{~Hz}$ is considerably lower than that of the polymer $(>100 \mathrm{G} \Omega$, Figure $5-10)$ and the coated aluminum samples $(\sim 100 \mathrm{M} \Omega$, Figure 5-19). In fact this low impedance indicates a poorly protective coating (probably only oxide in this case), and one would expect to see corrosion occurring quickly in aggressive solutions. 

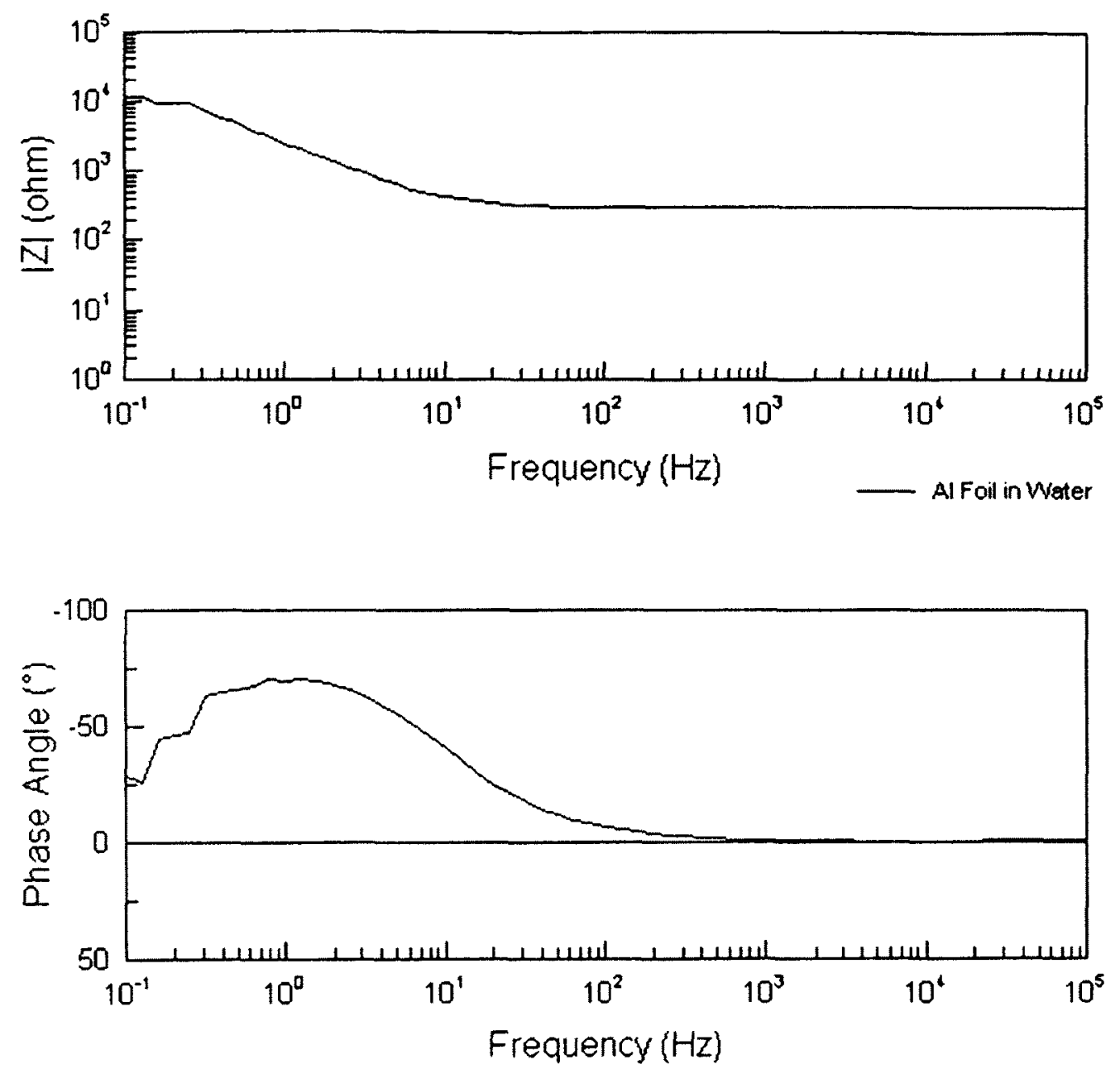

Figure 5-21 A Bode plot (using Solartron 1, VC, 34.0 $\pm 0.5 \mathrm{~cm}^{2}$ ) showing the impedance and phase angle for aluminum kitchen foil exposed to tap water. Compare this with data for an uncoated sample in Figure 3-14.

\subsubsection{Al Foil Exposed to $0.1 \mathrm{M} \mathrm{NaCl}$}

Upon exposure of the aluminum foil to a more corrosive solution than water, viz., $0.1 \mathrm{M}$ $\mathrm{NaCl}$ which is known to cause pitting $[14,15,16]$, there were immediately some notable differences in the behaviour of the EIS spectra. In line with the increased solution conductivity, conducting ions penetrated the coating/oxide and reached the underlying aluminum substrate. Due to the lower electrolyte resistance, the impedance associated 
with the capacitance is now dominant over a wider frequency range $(1 \mathrm{~Hz}-1 \mathrm{kHz})$ as compared to the sample in water $(0.2 \mathrm{~Hz}-10 \mathrm{~Hz})$. The impedance plot for the entire spectrum has dropped by orders of magnitude except for the frequency range of $1 \mathrm{~Hz}-10 \mathrm{~Hz}$ where it is measuring the value of the capacitance at the metal/oxide/electrolyte interface.

When water was the electrolyte, a transition from resistive to capacitive behavior was noted at $10 \mathrm{~Hz}$, while it occurred at $1000 \mathrm{~Hz}$ when the electrolyte was $0.1 \mathrm{M} \mathrm{NaCl}$. Also, a capacitive to resistive transition occurred at $0.1 \mathrm{~Hz}$ in water and $1 \mathrm{~Hz}$ in the $0.1 \mathrm{M}$ $\mathrm{NaCl}$ solution. Looking at the phase angle plot for the sample in $0.1 \mathrm{M} \mathrm{NaCl}$, as the frequency falls below $1 \mathrm{~Hz}$, a positive phase angle is observed in Figure 5-22. 

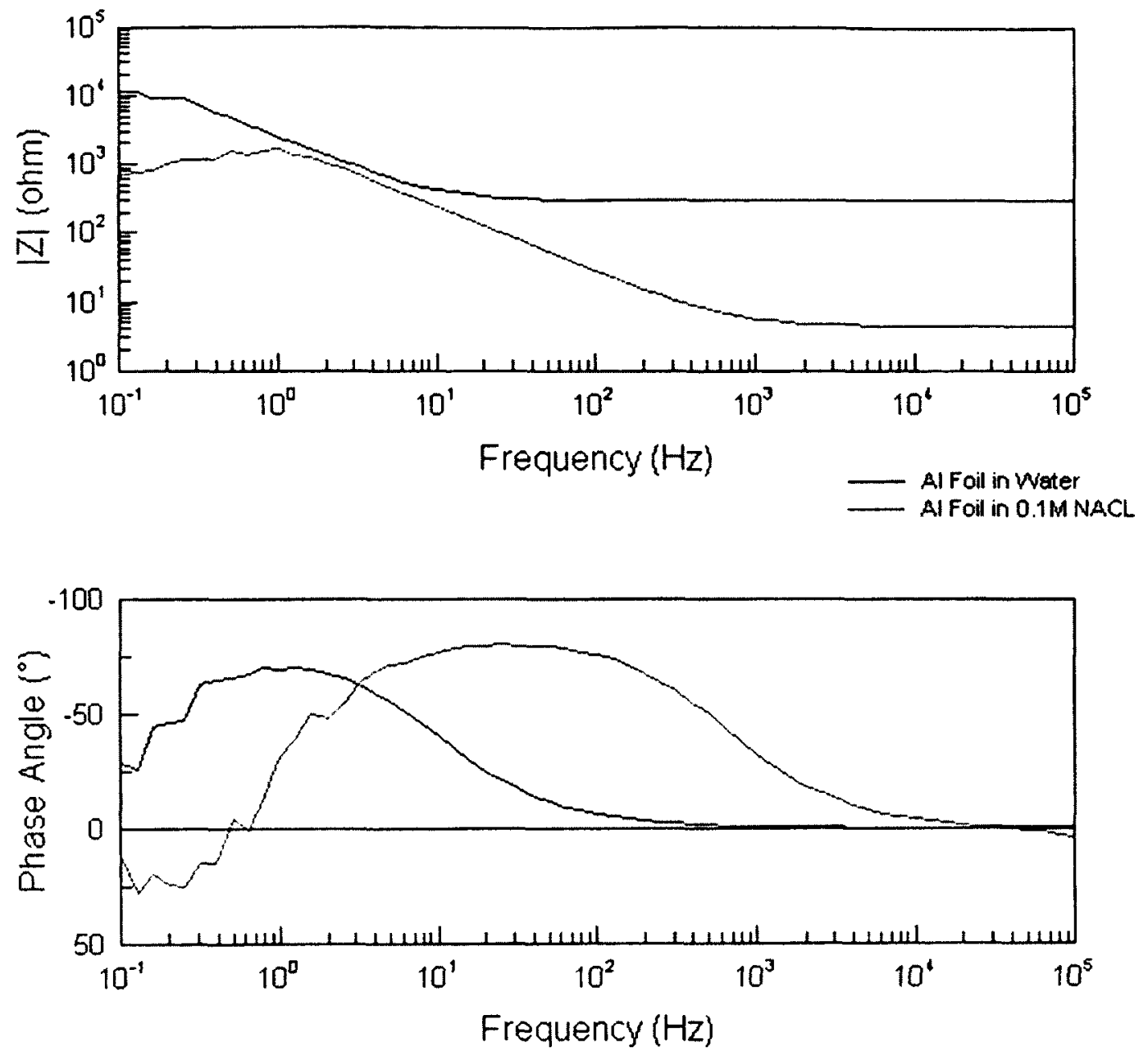

Figure 5-22 A Bode plot (using Solartron 1, VC, 34.0 $\pm 0.5 \mathrm{~cm}^{2}$ ) showing the impedance and phase angle for aluminum kitchen foil exposed to $0.1 \mathrm{M}$ NaCl.

\subsubsection{Al Foil Exposed to $1 \mathrm{M} \mathrm{NaCl}$}

To further investigate the changes occurring in the Bode plot, the electrolyte was changed to $1 \mathrm{M} \mathrm{NaCl}$. When the $\mathrm{Al}$ foil was exposed to this even more aggressive solution of $1 \mathrm{M}$ $\mathrm{NaCl}$ [15], there was yet another drop in impedance across the frequency spectrum: low frequency impedance was markedly lower. An increase in the magnitude of the positive phase angles was also observed; $+25^{\circ}$ in $0.1 \mathrm{M} \mathrm{NaCl}$ and $+50^{\circ}$ in $1 \mathrm{M} \mathrm{NaCl}[7,63-65]$. 
After 3 hours of exposure to $1 \mathrm{M} \mathrm{NaCl}$ there was a sudden drop in impedance at $0.4 \mathrm{~Hz}: 4 \mathrm{k} \Omega$ down to $<1 \mathrm{k} \Omega$, Figure $5-23$. The phase angle was observed to transition from capacitive to resistive at a higher frequency: $1 \mathrm{~Hz}$ to $10 \mathrm{~Hz}$ as shown in Figure $5-24$.

As time passed, the high frequency impedance $\left(>10^{3} \mathrm{~Hz}\right)$ was observed to increase slightly. This can be due to the fact that as the coating is getting breached, liquid is entering the narrow aperture between the coating and the aluminum substrate causing an increasing effective solution resistance to dominate over the coating capacitance. Another explanation for such behavior might be an increase in corrosion by-products in the electrolyte reducing its conductivity. The capacitance may also be decreasing as aluminum metal is consumed, changing the surface area.

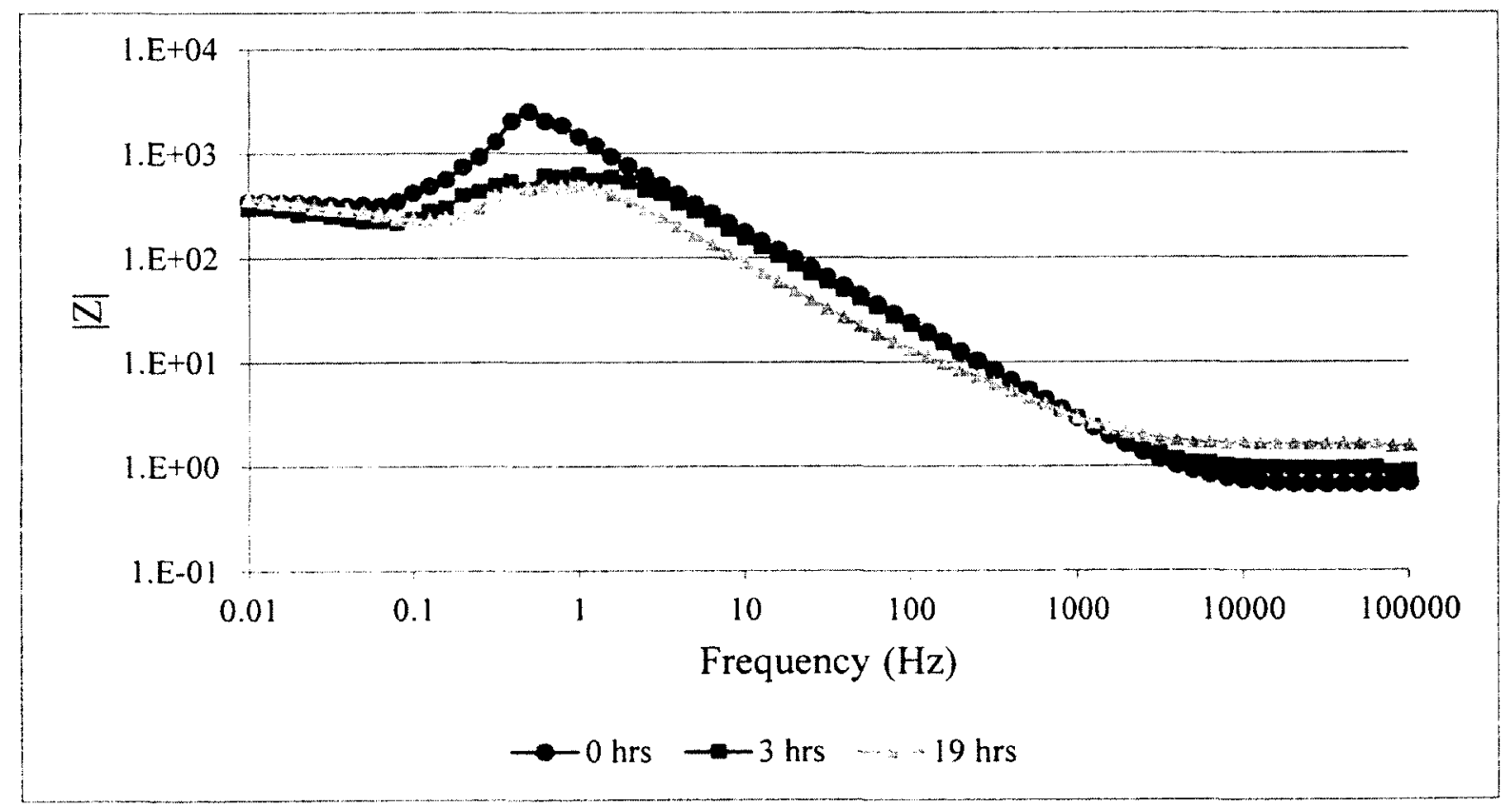

Figure 5-23 Impedance ( $\Omega$ ) measurements (using Solartron 1, VC, $34.0 \pm 0.5 \mathrm{~cm}^{2}$ ) for aluminum kitchen foil exposed to $1 \mathrm{M} \mathrm{NaCl}(\mathrm{pH} \mathrm{8})$. Note the increase in the high frequency impedance and drop in the low frequency impedance. 


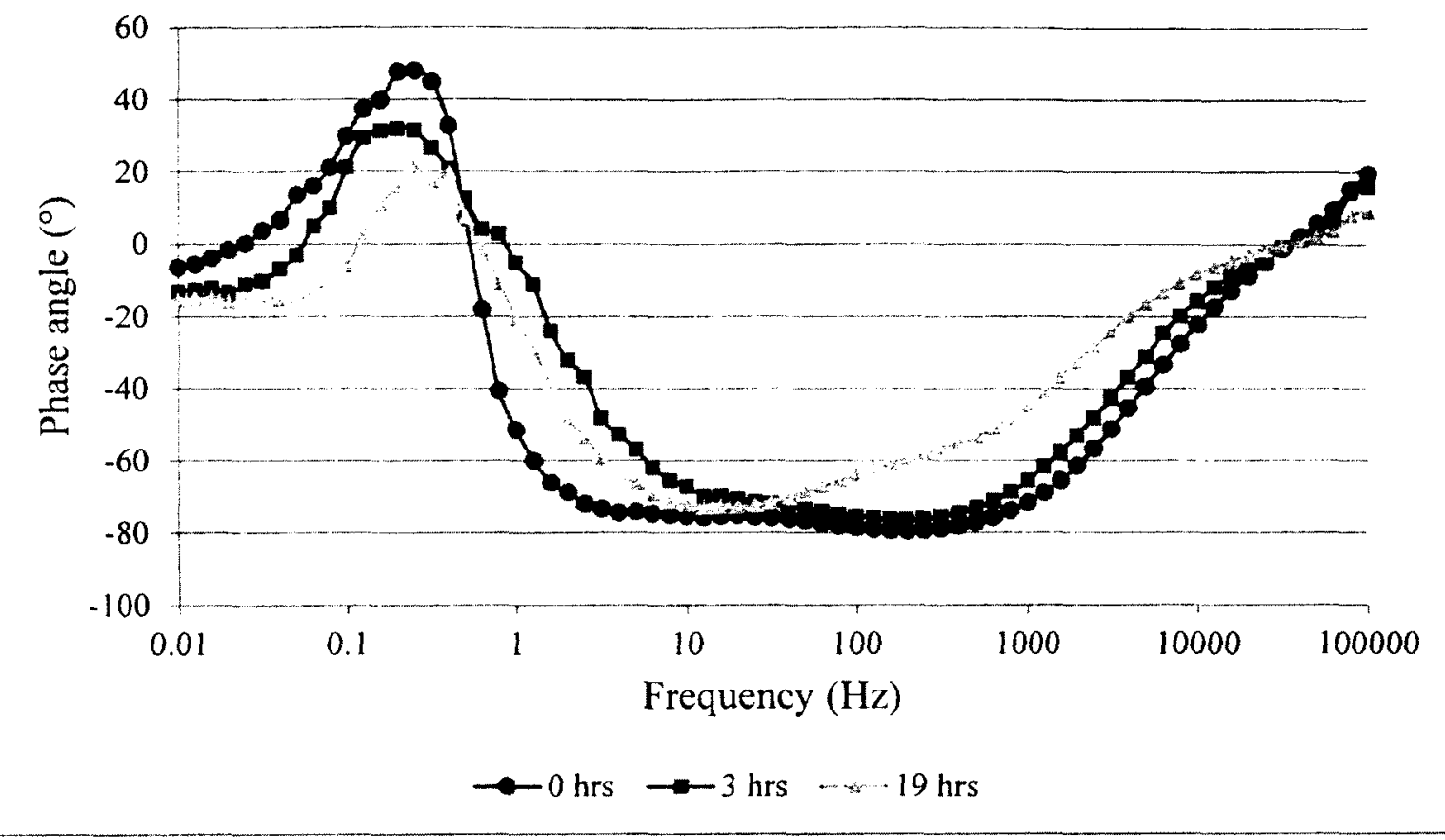

Figure 5-24 Phase angle measurements (using Solartron 1, VC, $34.0 \pm 0.5 \mathrm{~cm}^{2}$ ) for aluminum kitchen foil exposed to $1 \mathrm{M} \mathrm{NaCl}$ (pH 8). (the positive phase angle at the highest frequencies is attributed to inductance in the cables as previously discussed in section 5.2.2).

It is observed that the phase plot is becoming more complex when compares to passive non-corroding systems (c.f. Figure 5-21). No attempt has been made to model this obviously complicated system. 


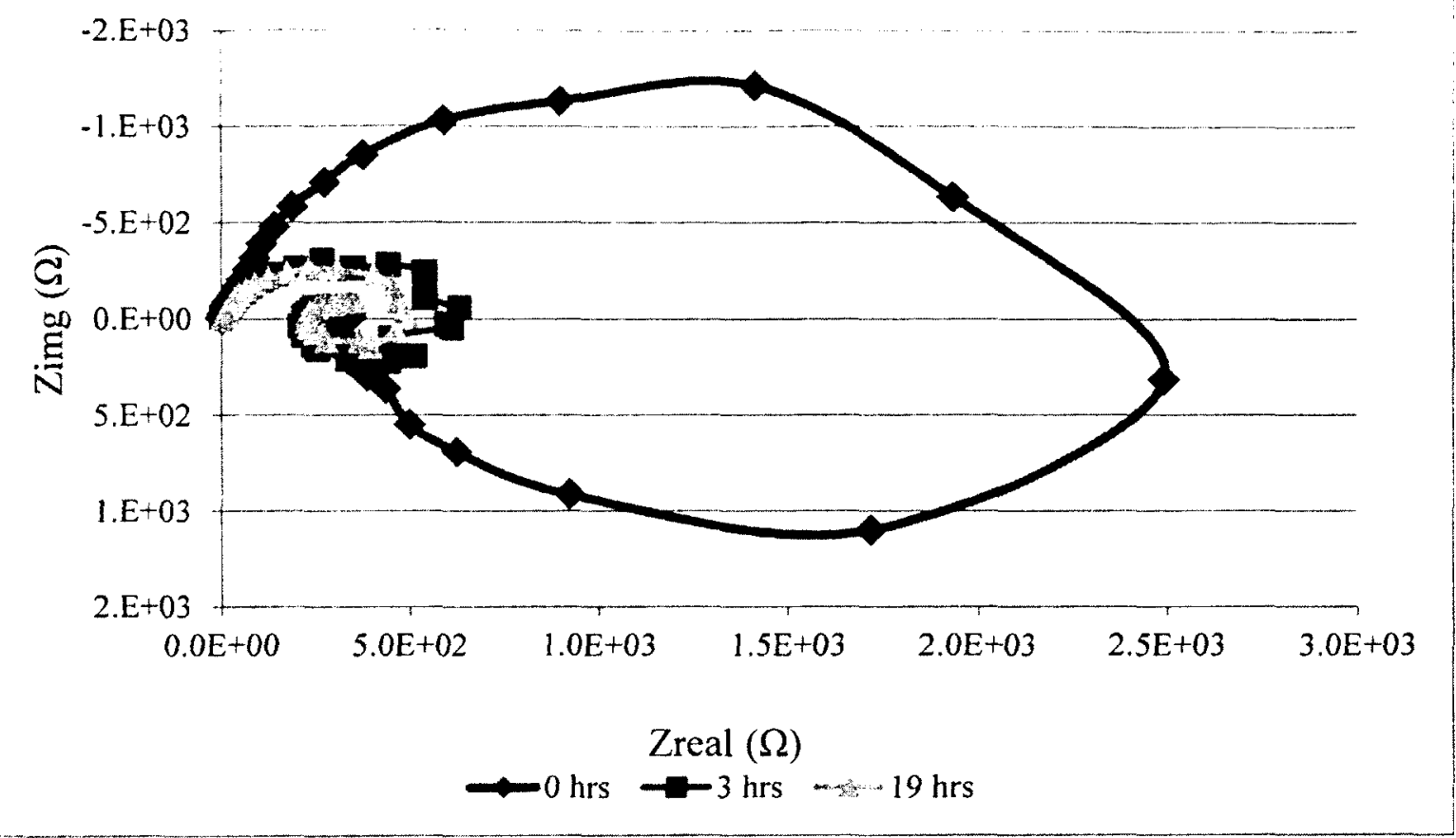

Figure 5-25 Nyquist plot (using Solartron 1, VC, 34.0 $\pm 0.5 \mathrm{~cm}^{2}$ ) for aluminum kitchen foil exposed to $1 \mathrm{M} \mathrm{NaCl}(\mathrm{pH} \mathrm{8})$. Note the inductive effects (positive imaginary impedance) appearing with sample degradation.

Upon examining the foil sample after experiments were complete, it was found to have many pinholes that completely penetrated the foil, Figure 5-26. It is believed that:

- the decrease in impedance at the lower frequencies;

- the capacitive to resistive transition occurring at higher frequencies;

- $\quad$ and the occurrence of positive phase angles (inductive effects)

are all indicative of chemical activity, viz., corrosion. To investigate these phenomena, DC polarization experiments were performed as explained in the next section. 


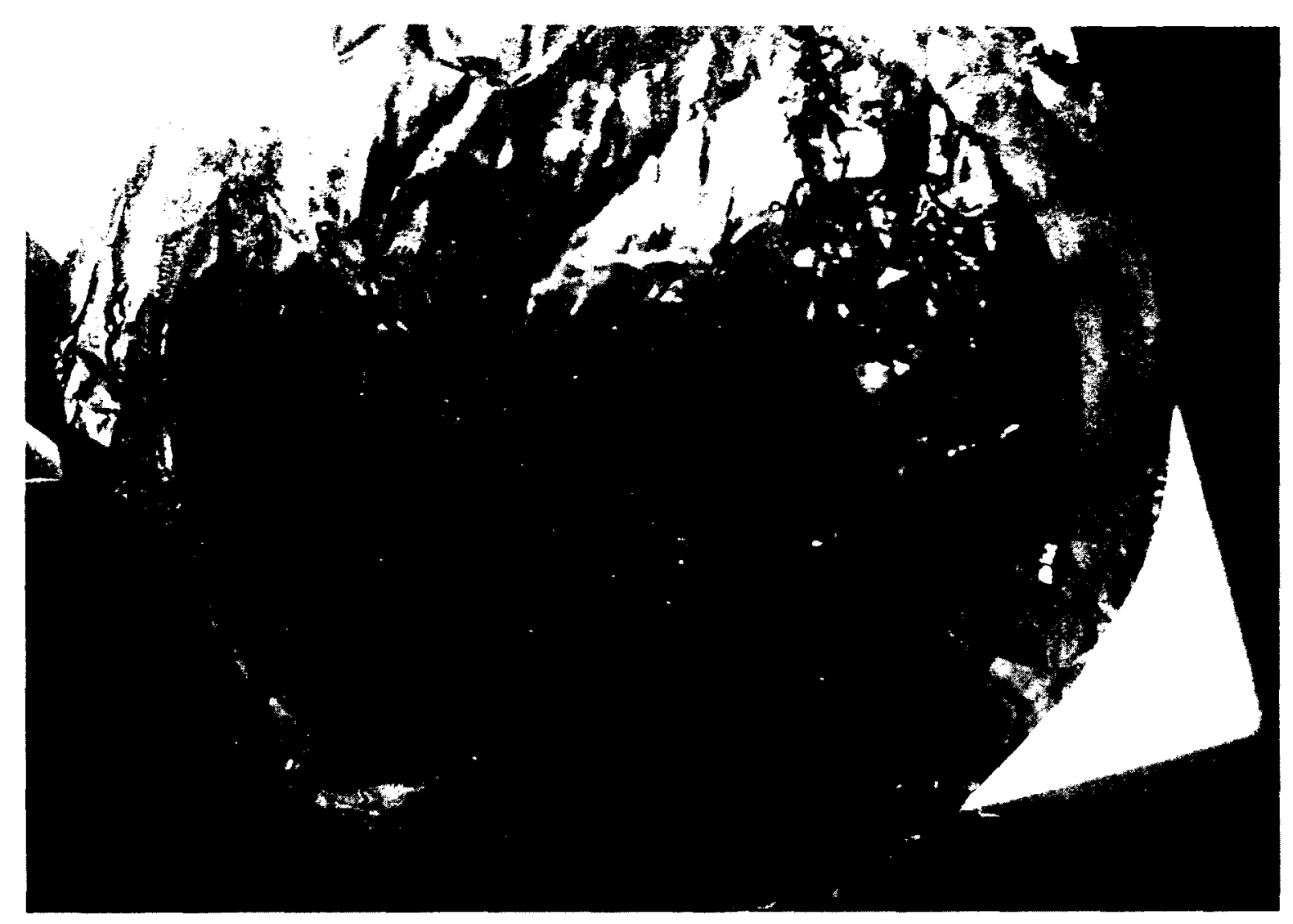

Figure 5-26 Photograph showing pinholes (numerous white dots) in aluminum foil after exposure to $1 \mathrm{M} \mathrm{NaCl}$.

\subsubsection{Polarization Experiments}

As pointed out in the previous section, the occurrence of inductive effects in the EIS data was attributed to corrosion. While running EIS scans, there is an option for applying a DC voltage, positive or negative, simultaneously with the AC voltage. The application of cathodic polarization, i.e., a negative voltage $(\sim-1 \mathrm{~V})$, pushes the system into the immunity region where corrosion essentially stops. This is discussed in section 3.3 . The application of such a voltage stopped the phase angle going positive and stopped the fall in impedance at low frequencies. The application of a small positive voltage $(\sim 1 \mathrm{~V})$ did the reverse (see Figure 5-27). It appeared that corrosion could be controlled by the 
application of small DC voltages to the foil $[7,15,16,66-68]$, indicating that a real inductor cannot be present in the circuit. Inductors cannot be turned off by applying a DC potential, and hence, a pseudo-inductive element is present. The disappearance of the positive phase angles during cathodic polarization strengthens the hypothesis that positive phase angles are indicative of corrosion.
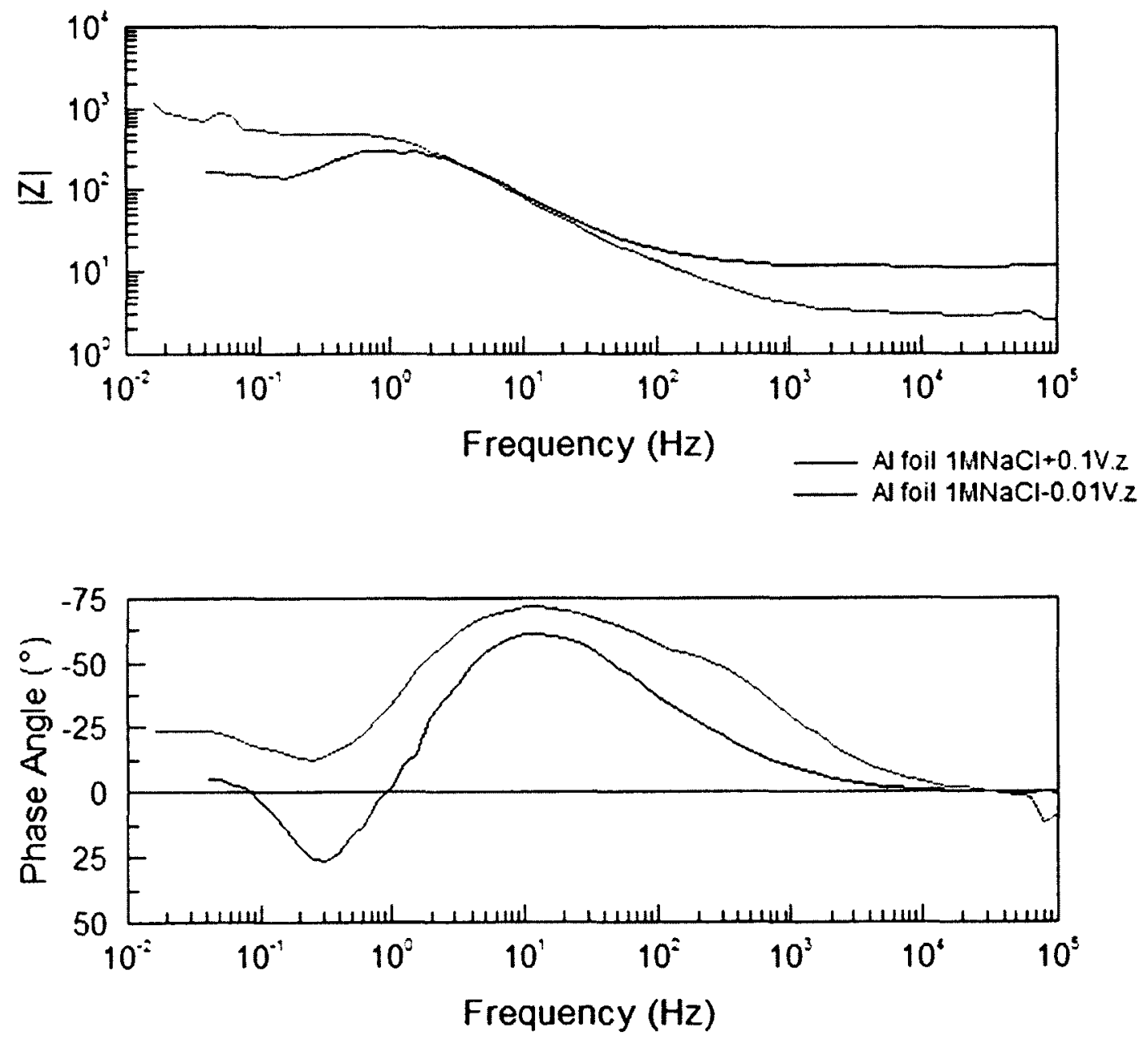

Figure 5-27 Bode plot (using Solartron 1, VC, 34.0 $\pm 0.5 \mathrm{~cm}^{2}$ ) showing the effects of the application of small DC voltages to aluminum kitchen foil in $1 \mathrm{M}$ $\mathrm{NaCl}$. 


\subsubsection{Results}

- The Bode plots for weak or flawed coatings are resistive at the high frequency range, capacitive at the mid frequency range and resistive at the low frequency end of the spectrum;

- For a weak or flawed coating, when the electrolyte concentration is increased, the impedance decreases across the frequency spectrum;

- With an increase in solution conductivity, the impedance attributed to the coating or oxide capacitance dominates over a wider frequency range;

- When the electrolyte is aggressive and the aluminum foil sample is corroding, a capacitive to resistive transition occurs at higher frequencies, $0.2 \mathrm{~Hz}$ for sample in water, $1 \mathrm{~Hz}$ for sample in $0.1 \mathrm{M} \mathrm{NaCl}$ and $10 \mathrm{~Hz}$ after 3 hours of exposure to $1 \mathrm{M}$ $\mathrm{NaCl}$;

- When a sample is actively corroding, positive phase angles are observed on the phase plot at low frequencies;

- A small DC voltage can be applied to turn corrosion on or off;

- When cathodic polarization is applied, positive phase angles disappear and an impedance that drops with lowering frequency is no longer observed;

- With prolonged exposure to $1 \mathrm{M} \mathrm{NaCl}$, the high frequency impedance was observed to slightly increase. 


\subsubsection{Conclusions}

By looking at all the results, it can be concluded that EIS can be used to determine the permeability of the coatings by varying the electrolyte concentration: the higher the concentration of electrolyte, the lower the impedance of a permeable coating.

Provided that the underlying metal is effectively protected from corrosion, the low-frequency impedance of a coated metal never decreases with decreasing frequency. In corroding systems, however, the impedance is lower at the lowest frequencies.

With the degradation of the aluminum foil samples, the capacitive-to-resistive transition frequency was observed to increase. As shown in section 2.5 , this is indicative of coating degradation. At the same time, there was a significant jump in the phase angle to positive values: inductive effects were observed. In the literature [7,63-65], such effects have been observed in EIS spectra of corroding metals. Although there are different views on how the inductive loop arises, in all cases it is agreed that it is associated with an active corrosion process. As observed in the DC polarization experiments, these inductive effects disappeared with the application of cathodic polarization. Hence, the conclusion that inductive effects at low frequencies indicate corrosion is widely supported. Corrosion was detected by EIS before it was visually observable.

In the EIS spectra, unexpected changes in the high frequency impedance were observed; the impedance slightly increased with exposure to $1 \mathrm{M} \mathrm{NaCl}$. These effects have not been fully explored, but possible explanations have been hypothesized: this effect may arise from a change in the dielectric constant of the coating or double layer on the aluminum surface due to solution entering between the coating and the aluminum 
substrate. This may also be explained by a reduction in the effective conductivity (higher impedance) due to an increase in corrosion by-products at the surface of the aluminum, and in flaws and pores.

Note that the aluminum foil samples have very weak protection as compared to the coated aluminum samples. When the aluminum foil samples are exposed to aggressive solutions, their coating is almost instantly penetrated with very minimal chemical resistance to the solution. The samples are fully protected at one instant and then fully breached the next instant: no intermittent corrosion taking place. The EIS signatures of intermittent corrosion can only be recorded by using samples with a strong coating which will resist the aggressive solutions for a period of time. This will be investigated in subsequent chapters.

\subsection{The Effect of Varying the Sample Size and Electrolyte}

\section{Concentration}

\subsubsection{Introduction}

After performing the aluminum foil experiments, it was important to investigate a few more parameters prior to collecting EIS data for actively corroding coated-aluminum samples. It was crucial to:

- Determine the effect of varying the sample surface area;

- Record differences in Bode Plot for Good and Poor Coatings;

- Confirm the effect of changing electrolyte concentrations on the EIS data. 


\subsubsection{Effect of Sample Size}

As previously stated in section 4.8 , two corrosion cells were available for collecting the EIS data. In the horizontal cell (HC), $1.0 \pm 0.1 \mathrm{~cm}^{2}$ of the sample is exposed to the electrolyte, while in the vertical cell (VC) a surface area of $34.0 \pm 0.5 \mathrm{~cm}^{2}$ is exposed. This

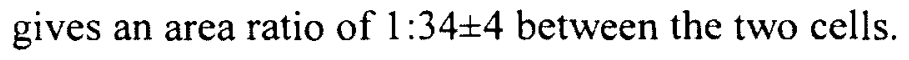

As explained in section 2.4 , the impedance of a capacitor is given by equation 2-15 and the capacitance, $C$, is calculated using equation 2-16. Hence, increasing the surface area by a factor of 34 should lead to impedance that is 34 times smaller for the samples in the vertical cell, compared to the horizontal cell.

Looking at Figure 5-19, EIS for a coated-aluminum sample in the vertical cell, the measured impedance is $\sim 100 \mathrm{M} \Omega$ at $0.1 \mathrm{~Hz}$. If the aforementioned prediction is right, a sample in the horizontal cell should have an impedance of approximately $3.4 \mathrm{G} \Omega$ at $0.1 \mathrm{~Hz}$. EIS data for the two corrosion cells were collected using Gamry 1 and Solartron 2; Figure 5-28. 


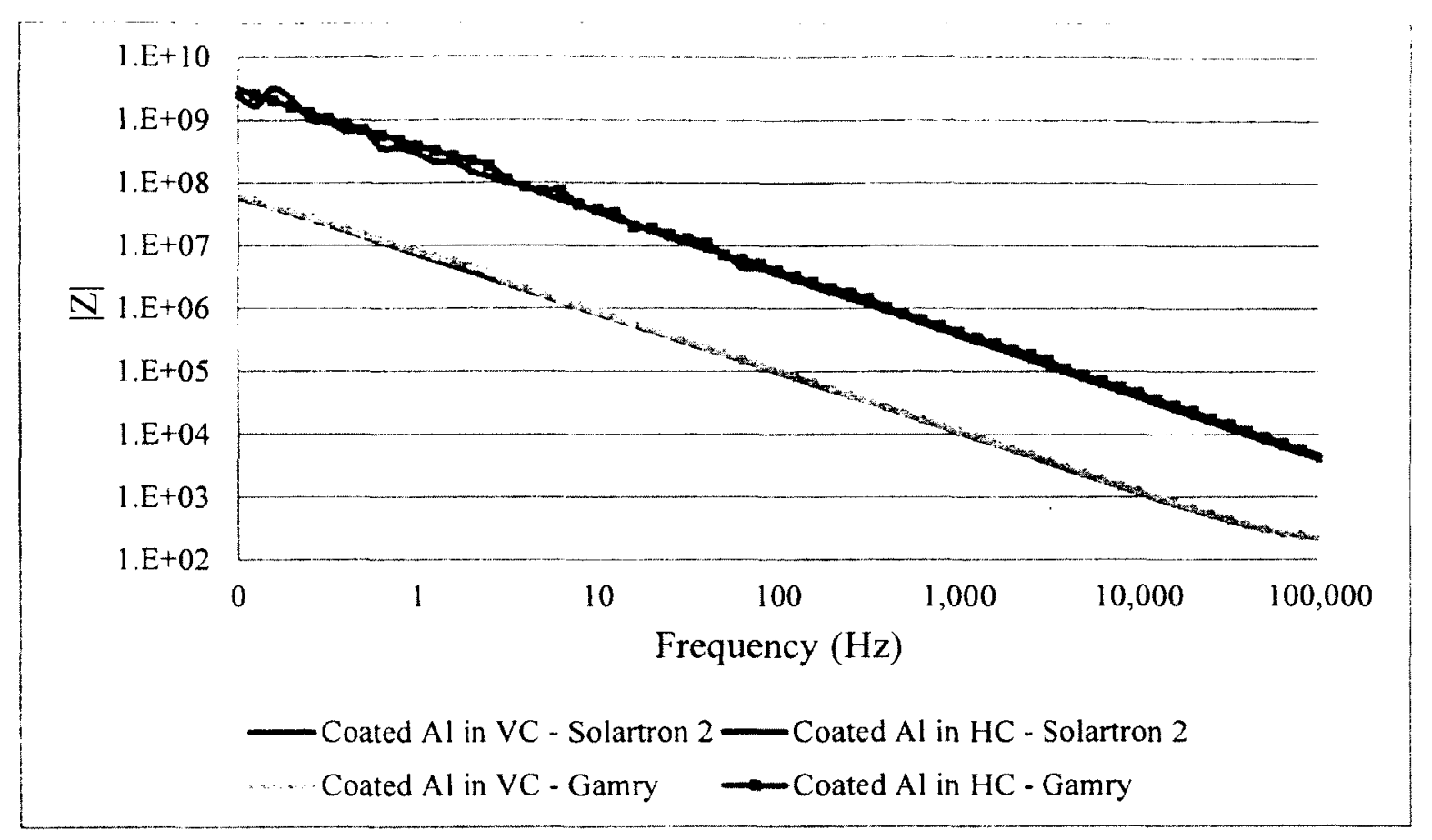

Figure 5-28 Impedance $(\Omega)$ measurements for coated-aluminum samples to show the effect of varying the sample surface area.

From Figure 5-28 it can be seen that, as predicted, the impedance value for an intact coated-aluminum sample is slightly higher than $3 \mathrm{G} \Omega$ at $0.1 \mathrm{~Hz}$. The ratio of the impedances, at all frequencies, was calculated and resulted in an average value of 37 for Solartron 2, and 40 for Gamry 1. In some of the experiments in section 6.4 , a shift in the impedance plot was observed indicating a change in the exposed surface area.

Using fitting software, a Randles circuit was fitted to the collected data and capacitance values were derived. The capacitances provided by the different machines were almost identical. The ratio of the capacitance values was also almost identical to the ratio of the impedances, see Table 5-2. 
Table 5-2. Calculated capacitance values, using fitting software, for the coated aluminum samples and their ratio.

\begin{tabular}{|c|c|c|c|c|}
\hline & \multicolumn{2}{|c|}{ Solartron 2 } & \multicolumn{2}{c|}{ Gamry 1 } \\
\cline { 2 - 5 } & Vertical cell & Horizontal cell & Vertical cell & Horizontal \\
& (VC) & (HC) & (VC) & cell (HC) \\
\hline Capacitance (F) & $1.73 \mathrm{E}-8$ & $4.57 \mathrm{E}-10$ & $1.72 \mathrm{E}-8$ & $4.35 \mathrm{E}-10$ \\
\hline Error $^{5}(\%)$ & 2 & 1.8 & $<0.1 \%$ & $<0.1 \%$ \\
\hline $\begin{array}{c}\text { Capacitance } \\
\text { Ratio (VC/HC) }\end{array}$ & \multicolumn{2}{|c|}{$\mathbf{3 7 . 8 \pm 1 . 4}$} & \multicolumn{2}{c|}{$\mathbf{3 9 . 5 \pm 0 . 1}$} \\
\hline
\end{tabular}

Even though the theoretical ratio of the areas is $1: 34 \pm 4$ and the experimental ratios of impedance values and capacitance values are 1:37.8 (Solartron 2) and 1:39.5 (Gamry 1), this implies that there is a linear relationship between the sample surface area ratio and the resulting impedance and capacitance measurements. A change in the working electrode surface area is reflected by a vertical shift in the impedance plot. The fact that there is not exact agreement between the theoretical and practical impedance ratios, may reflect the fact that the working electrode in the horizontal cell is more occluded and the counter electrode is much smaller than in the vertical cell, causing the horizontal cell to have a relatively higher impedance than expected.

Since a lower impedance implies a proportionally higher current, for a given $\mathrm{AC}$ voltage, the vertical cell will have higher currents and therefore much less signal noise than the horizontal cell [57,59], allowing the EIS instruments to measure comfortably high impedances in the vertical cell.

\footnotetext{
${ }^{5}$ The Error estimates are calculated by ZPlot (for Solartron instruments) and EchemAnalyst (for Gamry instruments); see section 4.7 .
} 
In this thesis, all plotted values of impedance will be in Ohms $(\Omega)$, and the crosssectional area of the sample will be given in the caption. To calculate the impedance for a sample with a new area, multiply the provided impedance values by the given sample area, and then divide by the new area. Impedance may sometimes have units of $\Omega$ $\mathrm{cm}^{2}$. In these instances, the measured values are multiplied by the cross-sectional area of the sample. In a similar way, capacitances are sometimes quoted in $\mathrm{F} / \mathrm{cm}^{2}$. However, in this thesis, changes in impedance will be measured after small defects are introduced into the coating samples. In these instances, very large currents can go through these tiny defects, i.e., the current density locally becomes extremely high, but because the active areas of the defects are not well known, current densities cannot be calculated, and impedances are not scalable by ratios of sample areas. Hence, absolute values of impedance for different defects cannot be directly compared.

\subsubsection{Differences in Bode Plot for Good and Poor Coatings}

Typically for a coating, at the highest frequency, the impedance measured is the solution resistance, $R_{s o l}$, in series with the essentially zero capacitive impedance of the coating, $C_{c}$ (see section 2.5); the slope of the Bode plot is zero, and the phase angle is zero. Then as the frequency decreases, the capacitive impedance increases and the solution resistance becomes negligible; the slope of the impedance plot approaches -1 (on a log-log scale). In the case of a polymer film that has resistance pathways tracking through it, e.g., porosity or flaws, the slope gradually changes from -1 to 0 . As the frequency further decreases, the capacitive impedance, $\mathrm{C}_{\mathrm{c}}$, becomes much higher than the parallel lower resistance $\mathrm{R}_{\mathrm{c}}$, and the impedance measured at the low-end of the frequency spectrum is the value of $R_{c}$. The impedance slope becomes 0 , and the phase angle is zero again. In Figure 5-10, the value 
of $R_{c}$ is so high, that it cannot be determined from the data; it would require measurements at much lower frequencies, probably $10^{-3} \mathrm{~Hz}$, which would take a very long time. The slope of the Bode impedance plot for the good coating in Figure $5-10$ is -1 (log-log scale) over the whole frequency range, whereas the slope for the minimal coating or oxide in Figure 5-21 is -1 for only a fraction of a decade. This is a way of differentiating between good and bad coatings.

From Figure 5-19, Figure 5-20 and Figure 5-28, it can be seen that an intact coated aluminum sample has a purely capacitive Bode plot in the frequency range of $10^{5} \mathrm{~Hz}-0.1 \mathrm{~Hz}$, with an impedance of $\sim 10^{8} \Omega-10^{9} \Omega$ at $0.1 \mathrm{~Hz}$. In the phase-angle plot, this is demonstrated by a phase angle of $-90^{\circ}$ in the frequency range of $10^{5} \mathrm{~Hz}-0.1 \mathrm{~Hz}$.

As explained in section 3.3.1, a chemically active metal will have a distinct polarization curve. In order to confirm the state of the samples at hand, active (flawed/corroding samples) versus inactive (intact samples), potentiodynamic experiments were performed after initial EIS data were collected.

First, potentiodynamic data for a high-impedance polymer, a chemically-inactive system, were collected using the horizontal cell and Solartron 2 as shown in Figure 5-29. The applied voltage was cycled between $-2 \mathrm{~V}$ and $1.5 \mathrm{~V}$, and the current density was monitored. 


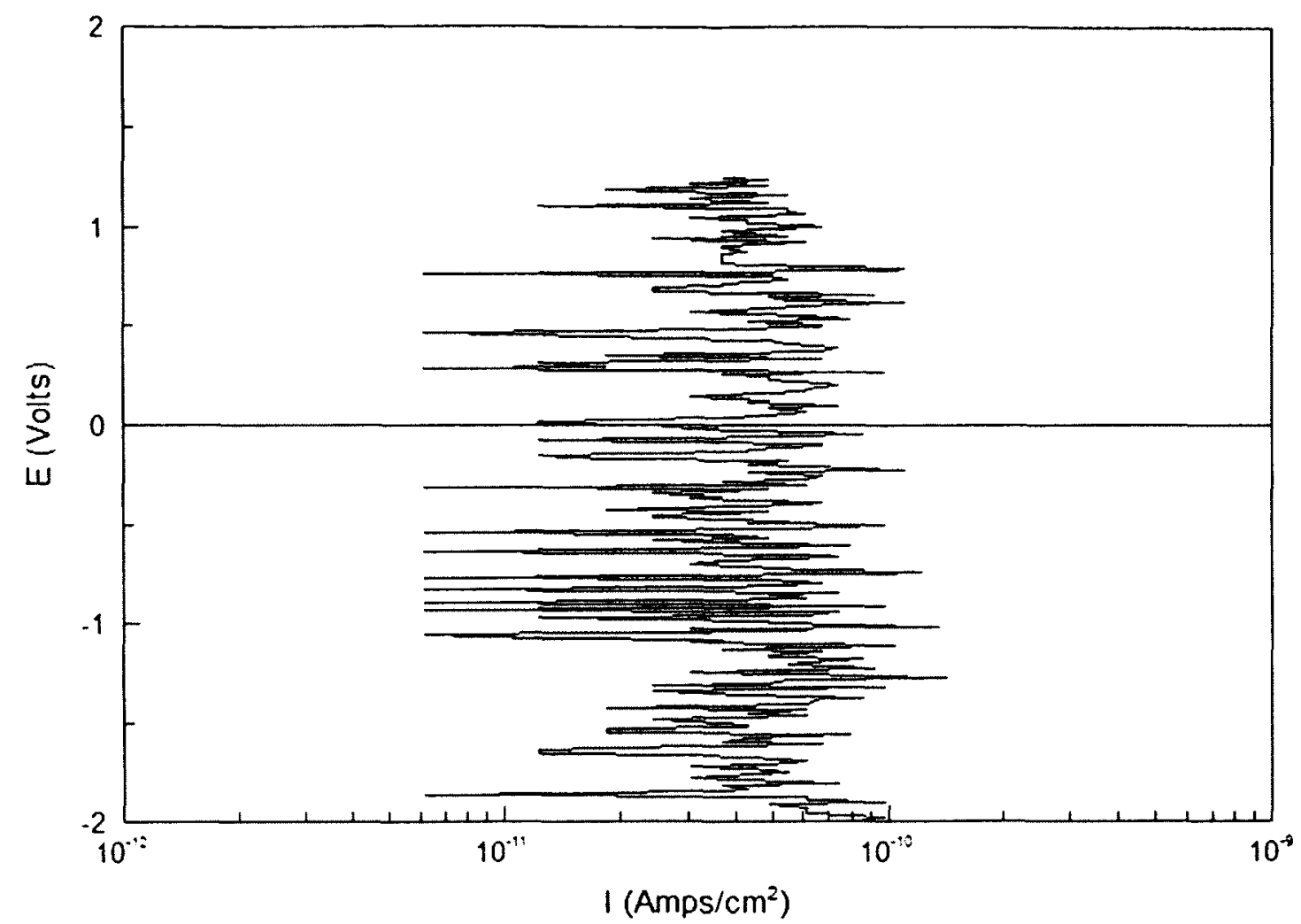

Figure 5-29 Potentiodynamic scan using Solartron 2 for the high-impedance epoxy polymer

With the changing voltage, there were no changes in the current density; a vertical line at $10^{-11} \mathrm{~A} / \mathrm{cm}^{2}-10^{-10} \mathrm{~A} / \mathrm{cm}^{2}$. This is similar to the potentiodynamic data collected for an open circuit, when leads are not connected to a cell, nor to each other. This gave a baseline for what a chemically inactive potentiodynamic scan looks like. It was expected that an intact coated-aluminum sample should produce a similar plot.

The high-impedance polymer sample was replaced with a coated aluminum sample and potentiodynamic data were collected. As can be seen from Figure 5-30, the potentiodynamic scan for the intact coated-aluminum sample is similar to the polymer sample. The current density is not changing with the applied voltage; it is producing a vertical line. This demonstrates that there are no chemical reactions taking place on the 
electrode surface. Therefore, an intact coated-aluminum sample should produce a vertical line at $\sim 10^{-10} \mathrm{~A} / \mathrm{cm}^{2}-10^{-11} \mathrm{~A} / \mathrm{cm}^{2}$ in a potentiodynamic plot.

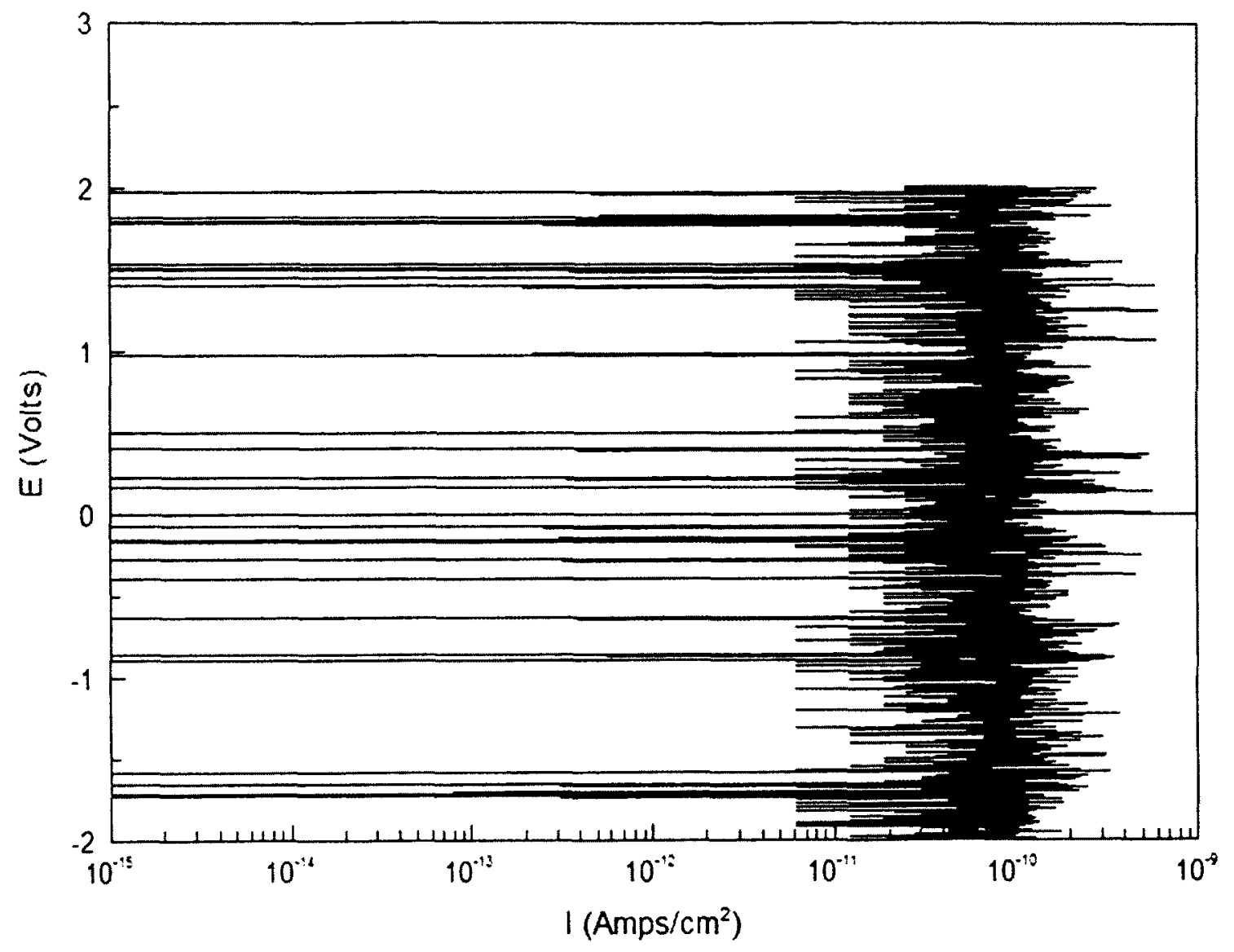

Figure 5-30 Potentiodynamic scan using Solartron 2 for an intact coated-aluminum sample.

\subsubsection{Effect of Changing Electrolyte Concentration}

To investigate the effect of changing the electrolyte concentrations on intact coatings, EIS data were collected for the intact sample of Figure 5-30 with two different solutions, $1 \mathrm{M} \mathrm{NaCl}$ and $1 \mathrm{M} \mathrm{HCl}$. Figure 5-31 shows the impedance and phase-angle plots for the sample in two different solutions. It can be seen that changing the solution has no effect on the EIS data. Since the coating is not flawed at the time of measurement, the solution 
has not penetrated the surface, has not reached the underlying substrate, and has not changed the impedance in any way.

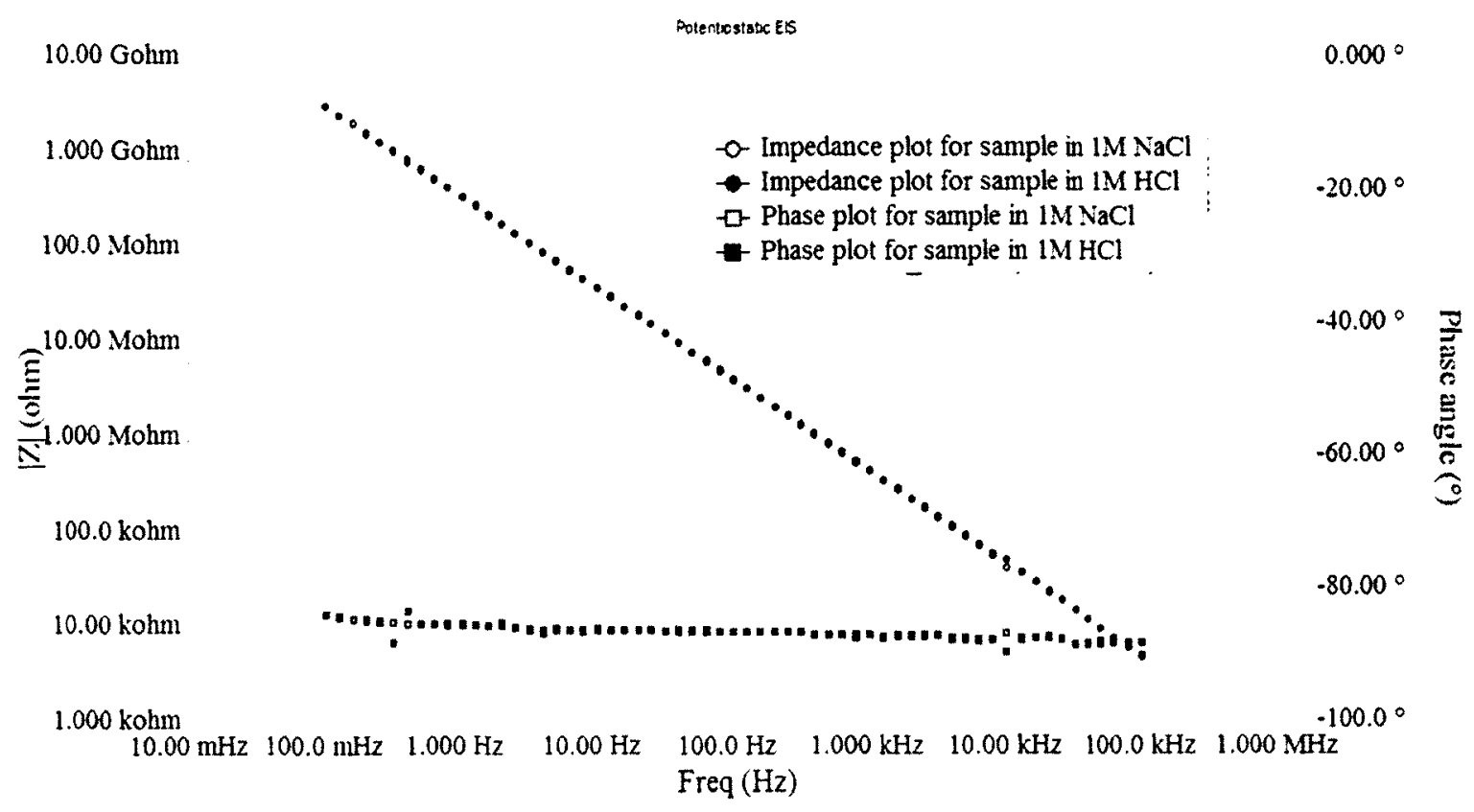

Figure 5-31 Bode plot using Gamry $1\left(\mathrm{HC}, 1.0 \pm 0.1 \mathrm{~cm}^{2}\right)$ for intact coated aluminum sample in two solutions, $1 \mathrm{M} \mathrm{NaCl}$ and $1 \mathrm{M} \mathrm{HCl}$.

\subsubsection{Conclusions}

- At neutral pH corroding aluminum readily passivates;

- By changing the sample surface area, the capacitance and impedance values change proportionally:

- Using a larger sample allows for better flaw detection which can be incorporated as a quality assurance technique;

- Using a smaller sample, the probability of the presence of surface defects is lower but this allows local testing of the coating quality;

- EIS can detect corrosion and its severity before it is visually observable; 
- An intact coated-aluminum sample can be identified by:

- a purely capacitive Bode plot in the frequency range of $10^{5} \mathrm{~Hz}-0.1 \mathrm{~Hz}$;

- an impedance of $\sim 10^{9} \Omega$ at $0.1 \mathrm{~Hz}$;

- the measured current density in a potentiodynamic plot does not change with the applied voltage; the plot will produce a vertical line at $10^{-10} \mathrm{~A} / \mathrm{cm}^{2}-10^{-11} \mathrm{~A} / \mathrm{cm}^{2}$;

- the Bode plot does not change when the electrolyte concentration is changed. 


\section{Chapter 6. Results for EIS of Coated Aluminum Samples}

\subsection{Introduction}

This chapter presents the results for the EIS experiments performed on the coated aluminum samples. The ability of EIS to differentiate between intact and flawed coatings was tested by investigating deliberately damaged coatings using $\mathrm{AC}$ (EIS) and DC (potentiodynamic) methods. To explore the effects of varying the $\mathrm{pH}$ and oxygen content on the performance of the coated aluminum samples, aerated and deaerated solutions were utilized (explained in section 4.3).

Sodium chloride solutions and hydrochloric acid solutions were utilized for the testing of the coated aluminum samples since they cause rapid corrosion and pitting of aluminum when the protective lacquer coating is breached, as discussed in section 3.3.2. As will be seen, this generates corrosion currents that are readily detected by our EIS instrumentation.

For the verification of the results, experiments were performed several times, using two different corrosion cells (see section 4.8). This chapter will only present one set of data for each condition; multiple experiments on different samples showed that all results were qualitatively reproducible (for example, see Figure 5-31).

\subsection{The Effect of Surface Defects}

Many of the samples had surface scratches. In order to see if EIS could detect flaws or imperfections in coatings, a number of experiments were performed using coatings that had been deliberately damaged by introducing a $0.5 \mathrm{~cm}$ long surface scratch. EIS data along with potentiodynamic data were collected before and after the scratch. 
Figure 6-1 shows the EIS data and Figure 6-2 show the potentiodynamic data for a sample in $0.1 \mathrm{M} \mathrm{NaCl}(\mathrm{pH} \mathrm{8)}$, before the scratch was introduced. As explained in section 5.4.3, the very high impedance at low frequencies $\left(>10^{9} \Omega\right.$ at $\left.0.1 \mathrm{~Hz}\right)$ and the constant phase angle of $-90^{\circ}$ over the complete frequency range are all traits of a purely capacitive intact sample. The very low measured current density in Figure 6-2, $10^{-10} \mathrm{~A} / \mathrm{cm}^{2}-10^{-11} \mathrm{~A} / \mathrm{cm}^{2}$, confirms that this sample was intact.
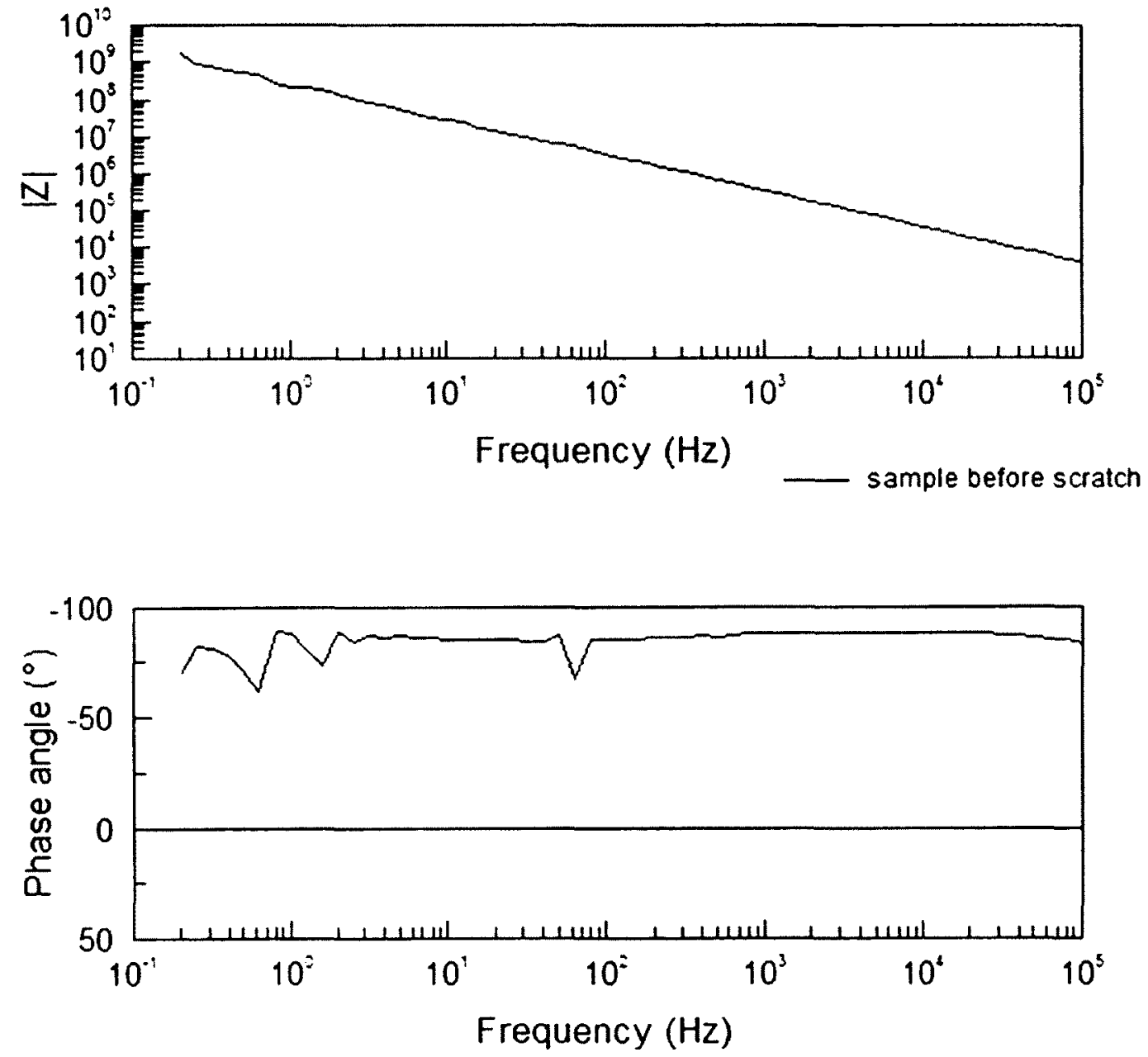

Figure 6-1 Bode plot (using Solartron 2, $\mathrm{HC}, 1.0 \pm 0.1 \mathrm{~cm}^{2}$ ) for a coated aluminum sample, immersed in $0.1 \mathrm{M} \mathrm{NaCl}(\mathrm{pH} \mathrm{8})$, before introducing a surface defect. Note the very high impedance at low frequencies, and the constant phase angle of $-90^{\circ}$, indicating that this is a good capacitor, over the complete frequency range. 


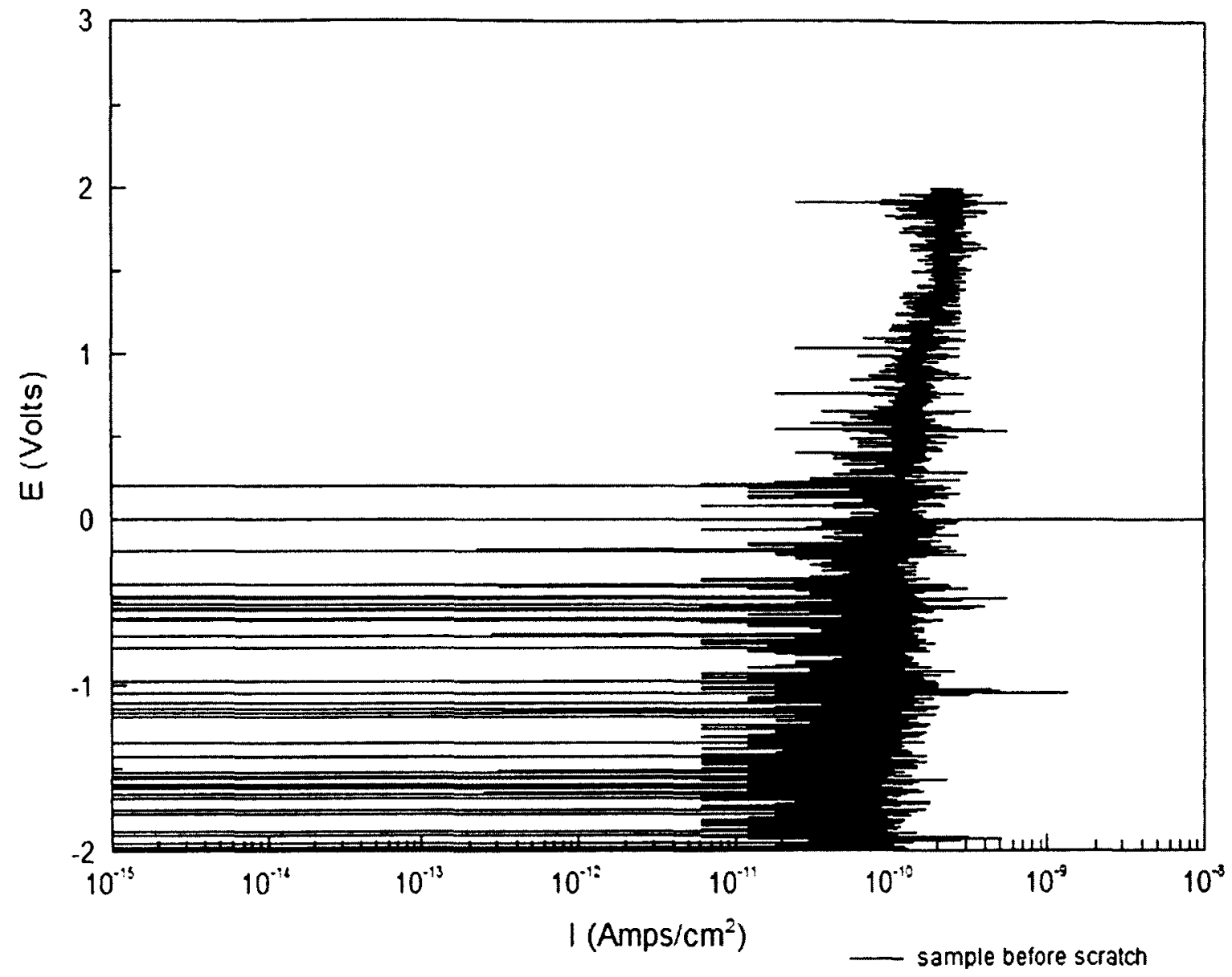

Figure 6-2 Potentiodynamic scan using Solartron $2\left(\mathrm{HC}, 1.0 \pm 0.1 \mathrm{~cm}^{2}\right)$ for a coated aluminum sample in Figure 6-1 (before introducing a surface defect). This is a low current density, at the detection level of the potentiostat.

After introducing a scratch onto the surface, EIS and potentiodynamic data were once again collected. The solution was then changed from $0.1 \mathrm{M} \mathrm{NaCl}(\mathrm{pH}$ 8) to $1 \mathrm{M}$ $\mathrm{NaCl}(\mathrm{pH}$ 8) to see the effect of changing electrolyte concentration on a flawed sample.

Examining the EIS data, it can be seen that the scratch caused dramatic changes. From Figure 6-3, it can be seen that the purely capacitive Bode plot (for the intact sample) is now showing Resistive-Capacitive-Resistive behaviour in both the impedance and phase-angle plots (for scratched sample). In the impedance plot, compared to the intact sample, a great drop in the impedance values across the whole frequency spectrum 
was observed. The impedance at $0.1 \mathrm{~Hz}$ has dropped from $\sim 3 \mathrm{G} \Omega$ down to $200 \mathrm{k} \Omega$ : 4 orders of magnitude.

In the phase plot, at high frequency, the sample is resistive, at mid frequency the sample is capacitive, and at the low frequency the phase angle crosses the zero line for a resistor and becomes positive $\left(+25^{\circ}\right.$ at $\left.0.1 \mathrm{~Hz}\right)$. This positive phase angle is observed when the frequency drops to $1 \mathrm{~Hz}$; similar to the kitchen aluminum foil experiment in Figure 5-22. This is indicative of actively corroding aluminum.

By switching to a more aggressive solution, $1 \mathrm{M} \mathrm{NaCl}$, there was yet another drop in the impedance, $10 \mathrm{k} \Omega$ at $0.1 \mathrm{~Hz}$; a drop of 1 order of magnitude. The same trend in the impedance plot, Resistive-Capacitive-Resistive, was also observed in the phase-angle plot.

The drop in impedance is due to the higher conductivity of $1 \mathrm{M} \mathrm{NaCl}$ as opposed to $0.1 \mathrm{M} \mathrm{NaCl}$; more $\mathrm{Cl}^{-}$ions are penetrating into the coating and reaching the metal substrate. The phase plot is similar for both solutions, but an even more positive phase angle, $+50^{\circ}$, is observed when using $1 \mathrm{M} \mathrm{NaCl}$ for the electrolyte, as opposed to $+25^{\circ}$ for $0.1 \mathrm{M} \mathrm{NaCl}$; this is comparable with corroding aluminum foil in Figure 5-24. 


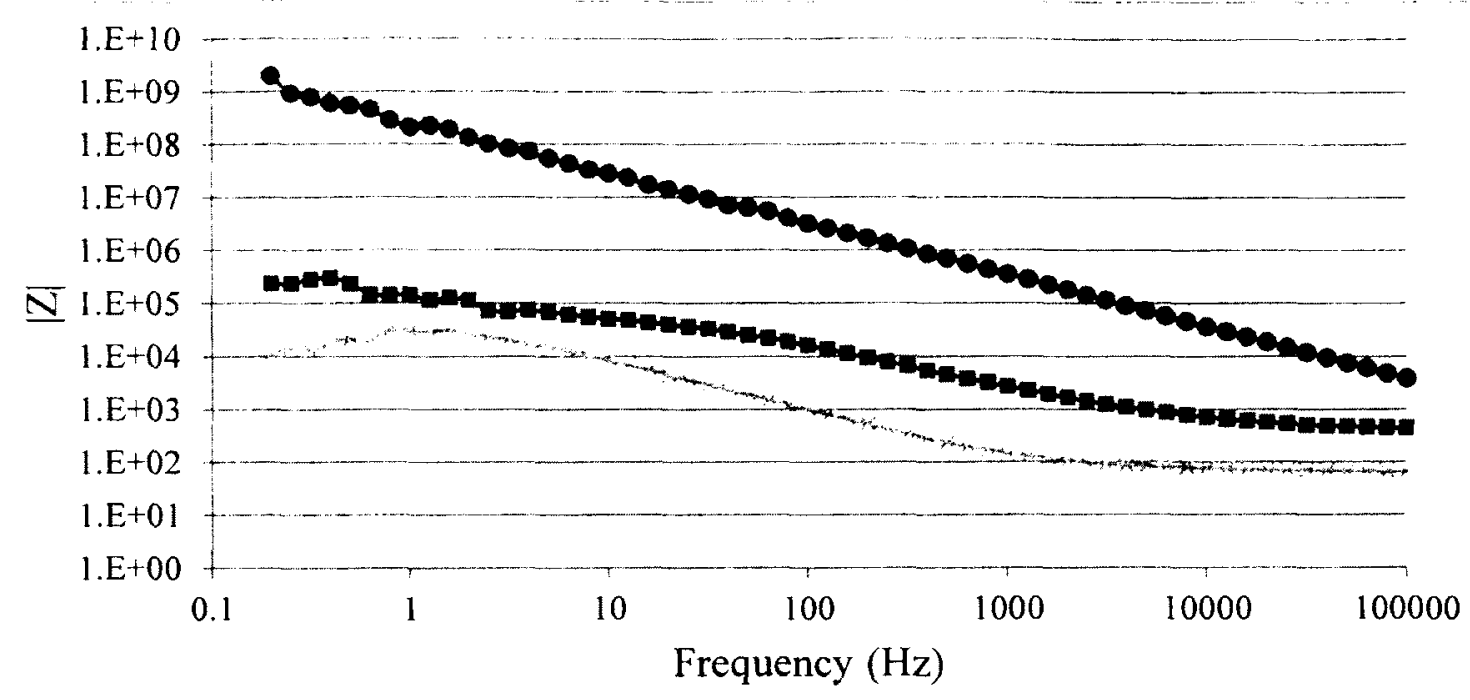

Sample before scratch $\rightarrow-0.1 \mathrm{M} \mathrm{NaCl}$ with scratch $-\rightarrow-1 \mathrm{M} \mathrm{NaCl}$ with scratch

Figure 6-3 Impedance ( $\Omega$ ) plot using Solartron $2\left(\mathrm{HC}, 1.0 \pm 0.1 \mathrm{~cm}^{2}\right.$ ) for a coated aluminum sample before a surface scratch in $0.1 \mathrm{M} \mathrm{NaCl}(\mathrm{pH}$ 8), and after introducing a surface scratch (in $0.1 \mathrm{M} \mathrm{NaCl}(\mathrm{pH} 8)$ and $1 \mathrm{M} \mathrm{NaCl}$ (pH 8)). Note the drop in impedance by several orders of magnitude after the scratch, and by changing solution concentration.

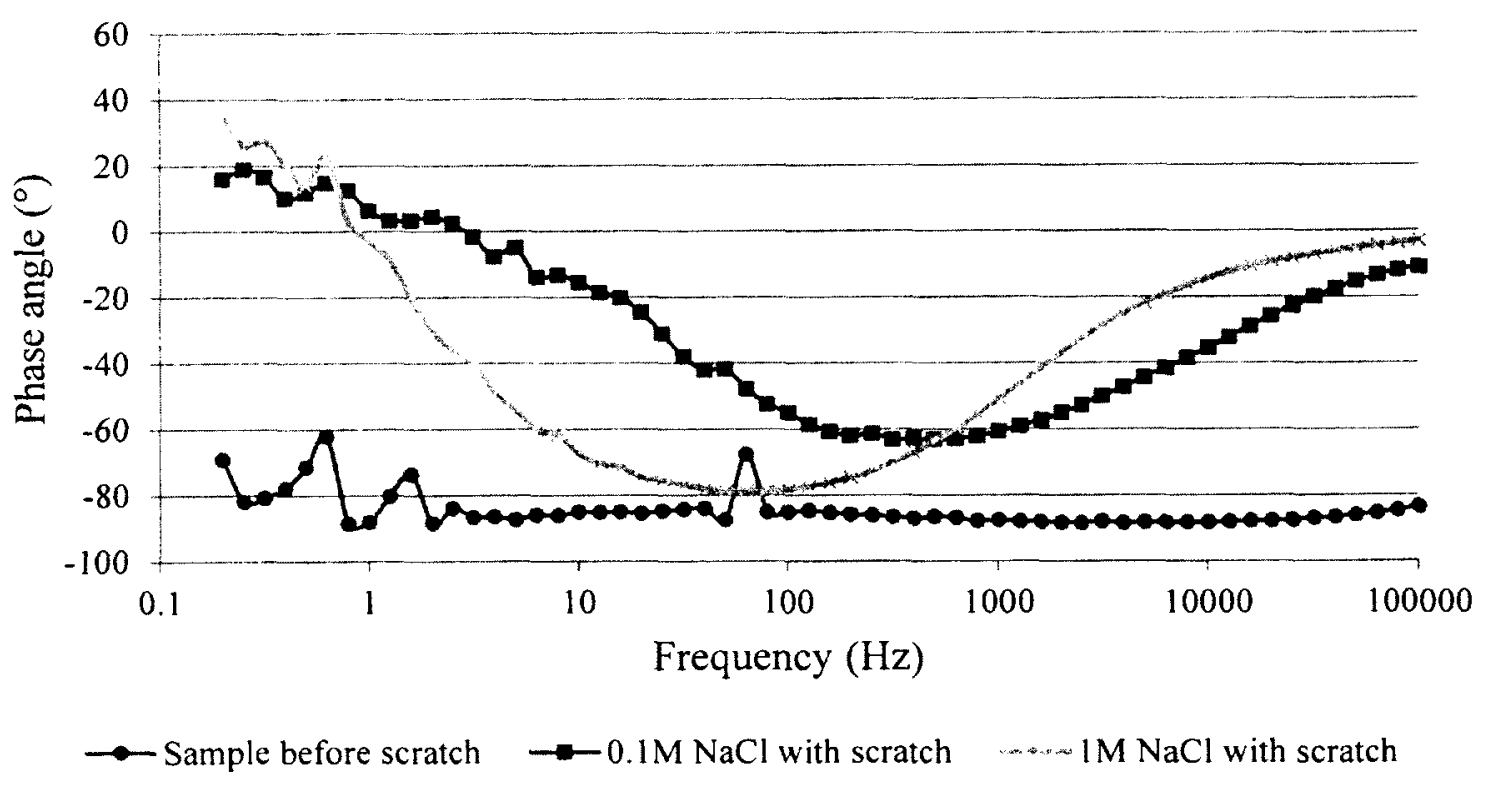

Figure 6-4 Phase angle plot using Solartron $2\left(\mathrm{HC}, 1.0 \pm 0.1 \mathrm{~cm}^{2}\right)$ for a coated aluminum sample before and after a surface scratch, and after changing the electrolyte from $0.1 \mathrm{M} \mathrm{NaCl}(\mathrm{pH} \mathrm{8})$ to $1 \mathrm{M} \mathrm{NaCl}(\mathrm{pH} \mathrm{8})$. Note that the magnitude of the positive phase angle increased with increasing solution conductivity. 


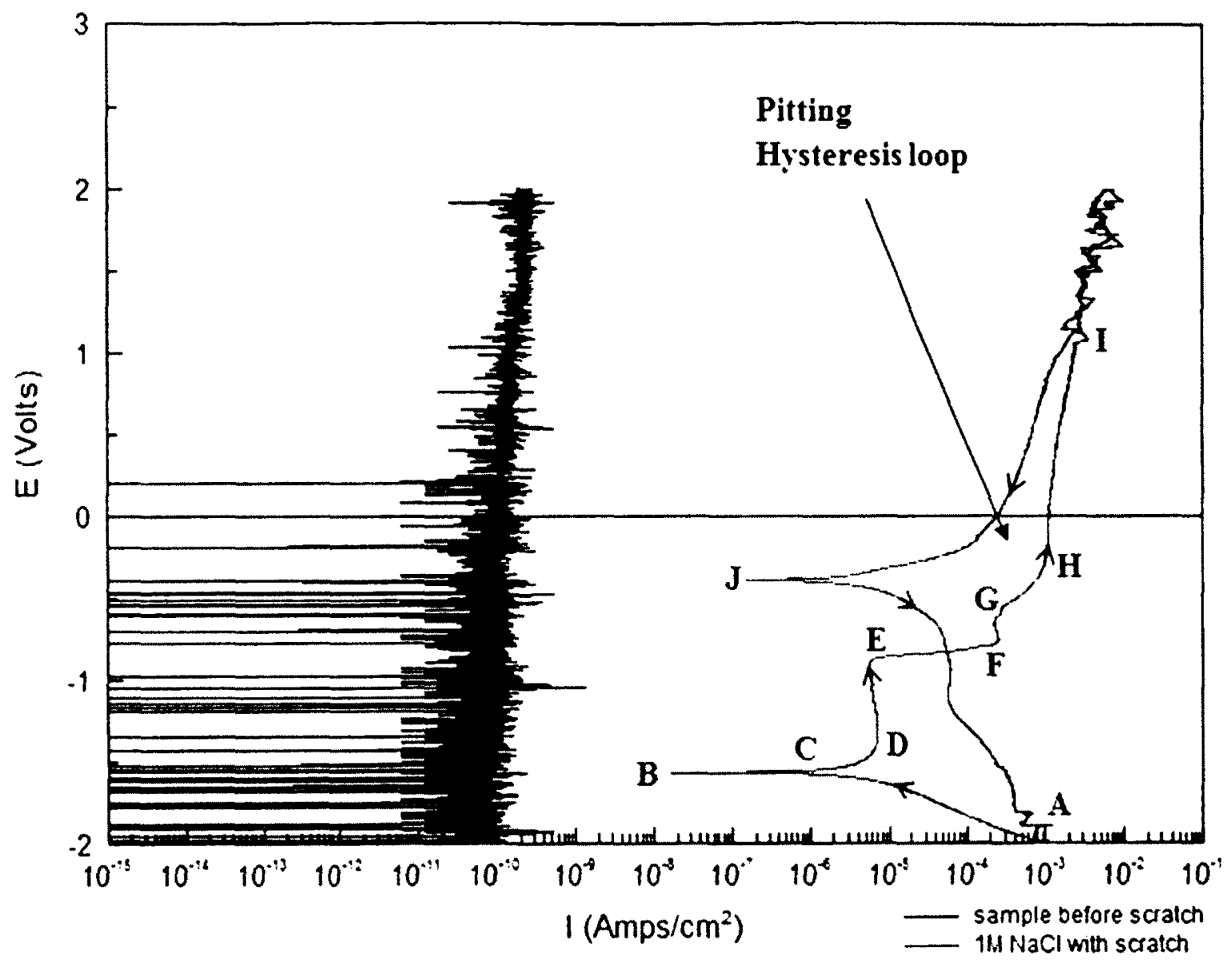

Figure 6-5 Potentiodynamic scan using Solartron $2\left(\mathrm{HC}, 1.0 \pm 0.1 \mathrm{~cm}^{2}\right)$ for a coated aluminum sample, in $0.1 \mathrm{M} \mathrm{NaCl}(\mathrm{pH} 8)$ before scratch and in $1 \mathrm{M}$ $\mathrm{NaCl}(\mathrm{pH} 8)$ after a surface scratch.

Looking at the potentiodynamic data, Figure 6-5, there were also dramatic changes. The potentiodynamic curve after the scratch is that of a chemically active sample (explained in section 3.3.1). Point A demonstrates the starting point of the potentiodynamic curve. Since the starting potential, $-2 \mathrm{~V}$, is lower than the corrosion potential, point $\mathrm{B}$, the polarization curve follows a cathodic path until the applied potential is equal to the corrosion potential.

As the potential is increased from the corrosion potential (point B), the measured current density increases due to an increase in the rate of corrosion. As the potential 
reaches point $\mathrm{C}$, the critical passivation potential, an oxide film forms on the metal surface and the metal transitions from an active state to a passive state. Due to the formation of the oxide film causing passivity of the metal, the current density starts to decrease at the Flade Nose/potential (Point D).

The Flade potential is generally close to the critical passivation potential, at which the metal dissolution occurs at a constant rate and the dissolved film is immediately replaced by a new film and a net balance is maintained between the dissolution and film reformation; the current density becomes virtually independent of potential and remains virtually stationary. At this point the scratch is covered by an imperfect passive oxide.

As the potential is farther increased, a sudden and large increase in the current density is observed at point $\mathrm{E}$. Typically such a sudden increase in current density is indicative of pitting [16], starting at the pitting potential. The current density then reaches point $F$, where there appears to be a second Flade Nose. The current density once again reaches a steady value as a function of potential. The presence of this feature means that the alloy has a higher finite corrosion rate at this potential (factor of 100 higher than at E), although the surface again appears to be undergoing some type of passivation process or chemistry change [69-71]. Since aluminum does not change valency (it is either 0 or 3 ) this most likely indicates the formation and repassivation of metastable pits.

As the potential reaches point $\mathrm{G}$, the current density rises again, and is now in excess of $1 \mathrm{~mA} / \mathrm{cm}^{2}$ indicating a rapidly corroding sample. As point $H$ is reached, the current density is observed to be no longer increasing with the potential. Spiking of the measured current density starts to appear as the applied potential approaches a value of $+1 \mathrm{~V}$, point $\mathrm{I}$; this is indicative of stable pitting occurring in the sample. 
The scan is reversed when the potential reaches a value of $+2 \mathrm{~V}$. The loop created between the forward and reverse curves is known as the pitting hysteresis loop. The hysteresis is created by the current density difference between the forward and reverse portions of the scan at the same potential. It is a result of the change in the corroding surface structure, which occurred during the increase in potential.

The hysteresis loop can be classified as either positive or negative. Sometimes, polarization to more noble potentials makes the surface more passive so that when the same potential is reached on the return portion of the scan, the current density is lower because the surface is more passive than during the forward scan. Hence, the current density is lower and the reverse scan occurs to the left of the forward scan. This is a "negative" hysteresis loop [41] and is what we have in Figure 6-5.

A "positive" hysteresis loop results from the opposite effect. Polarization to more noble potentials causes a decrease in passivity, sometimes by the initiation of localized corrosion (pitting), so that when the same potential is reached on the return portion of the scan, the current density is greater because the surface is less passive. Hence, the current density is greater, and the reverse curve is to the right of the forward scan. This phenomenon is usually a reflection of localized corrosion such as pitting or crevice corrosion.

From a practical standpoint, a negative hysteresis usually signifies that the alloy will be more resistant to localized corrosion than one which shows a positive hysteresis [41]. A large positive hysteresis actually reflects greater difficulty in repassivating an actively corroding surface as the potential is decreased back toward the corrosion potential. The larger is this positive hysteresis loop, the greater is the work required to 
restore passivity, and usually the greater is the risk of localized corrosion [69-71]. A large negative hysteresis loop indicates that the surface is passivating more rapidly than the original surface before an anodic voltage was applied, and this surface is likely to show less corrosion and less pitting than the original sample, and less than its positive-hysteresis counterpart.

In Figure 6-5, after the applied potential is reversed, for a short time the return scan follows the same spiking path as the upward scan, with no sign of repassivation, indicating active corrosion. When the potential approaches $+1 \mathrm{~V}$, point $\mathrm{I}$, the current density is observed to decrease in magnitude, indicating some repassivation. This is followed by a negative hysteresis loop, confirming that some passivation is taking place on the surface. Along the return curve, the current density starts to decrease, due to repassivation, until it reaches point $\mathrm{J}$ known as the repassivation potential (or protection potential) [69-71].

The potentiodynamic plot shown in Figure 6-5 is very complicated, however, it can be concluded that the scratch in the coating allows the electrolyte to reach the metal substrate; chemical reactions, consisting of both general and localized corrosion (metastable/stable pitting), are taking place. It should be noted, however, that the pitting only occurs at higher impressed voltages. At C and D, close to the corrosion potential, B, general corrosion and passivation occur.

By looking at Figure 6-6, the Nyquist plot prior to introducing the surface scratch, a virtually vertical line is observed. Such a curve corresponds to a capacitive element; once again confirming that the sample was intact [12]. 


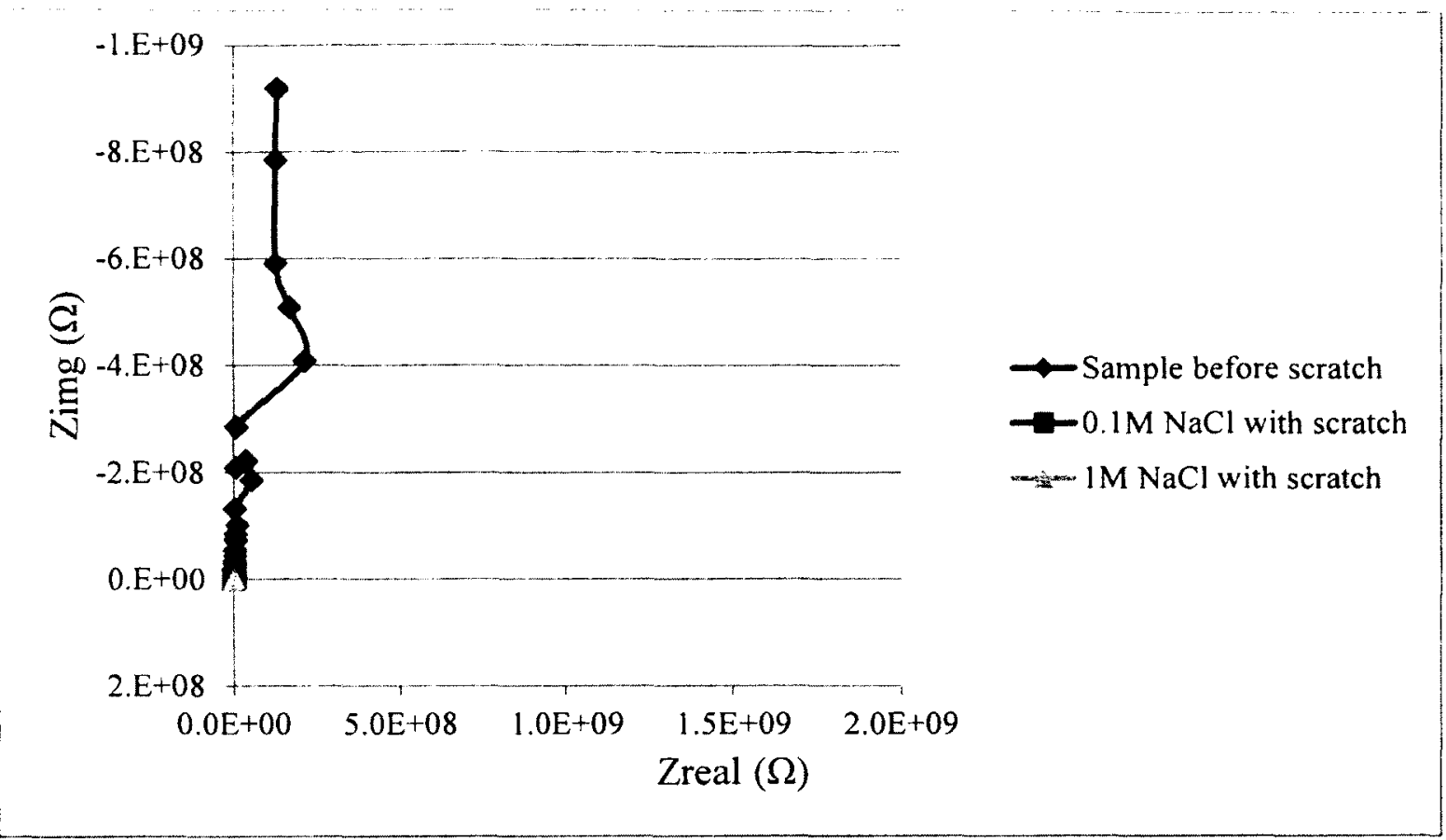

Figure 6-6 Nyquist plot using Solartron $2\left(\mathrm{HC}, 1.0 \pm 0.1 \mathrm{~cm}^{2}\right)$ for a coated aluminum sample before and after a surface scratch in $0.1 \mathrm{M} \mathrm{NaCl}(\mathrm{pH} 8)$ and in $1 \mathrm{M} \mathrm{NaCl} \mathrm{(pH} \mathrm{8)} \mathrm{after} \mathrm{a} \mathrm{surface} \mathrm{scratch.} \mathrm{The} \mathrm{unscratched} \mathrm{sample}$ behaves like a capacitor [12]. See also Figure 6-7 for a better view of the plots for the scratched sample.

The recorded Nyquist plots, after the introduction of the surface scratch, are not clearly visible in Figure 6-6 because of the difference in magnitudes of the recorded impedances; these curves are enlarged and shown in Figure 6-7.

The Nyquist plots of the scratched samples are no longer straight vertical lines, but rather form circular plots which indicate a vulnerable corroding metal substrate, as a result of a damaged coating (explained in section 2.5). By changing the solution concentration, it is observed that the magnitude of the Nyquist plots decrease when the solution conductivity is increased. In this case, both the real and imaginary impedances have decreased indicating less protection of the metal by the coating. 
It is also important to take note of the curves extending below the horizontal axis and looping around it; these are pseudo-inductive effects. Such occurrences are attributed to active corrosion occurring on the metal substrate. Such pseudo-inductive effects have been observed whenever corrosion happens. This was shown earlier in corroding aluminum foil (see Figure 5-25). Such pseudo-inductive effects also have low-frequency positive phase angles in Bode plots.

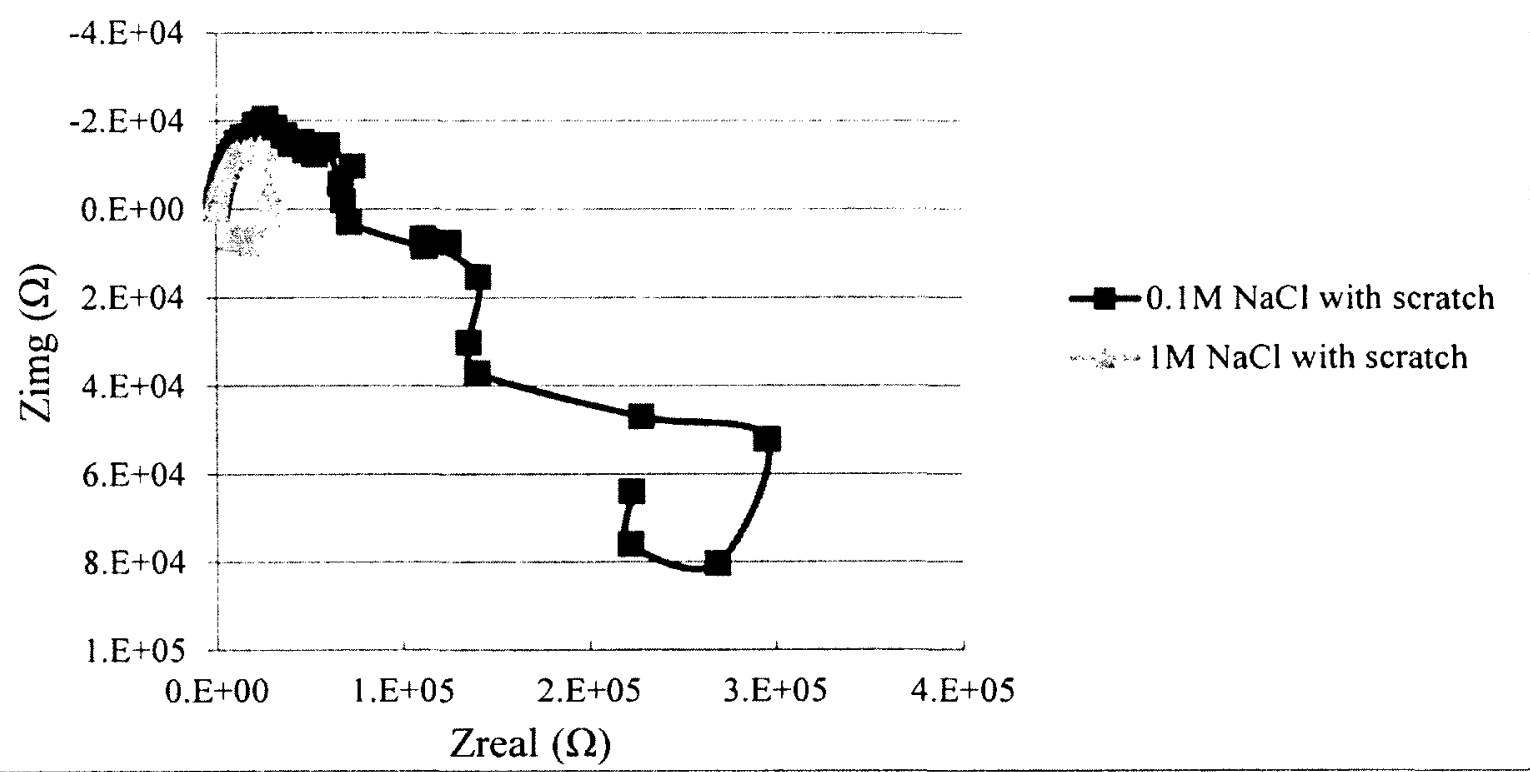

Figure 6-7 Nyquist plot using Solartron $2\left(\mathrm{HC}, 1.0 \pm 0.1 \mathrm{~cm}^{2}\right)$ for a coated aluminum sample after a surface scratch in $0.1 \mathrm{M} \mathrm{NaCl}(\mathrm{pH} 8)$ and in $1 \mathrm{M} \mathrm{NaCl}$ (pH 8). Note the pseudo-inductive effects (positive Zimag) observed in the plot. Note the decrease in magnitude of both Zreal and Zimg when the electrolyte was more concentrated.

\subsection{Experiments in Aerated Solutions}

\subsubsection{Introduction}

This section presents the results of a series of studies in which coated aluminum samples are exposed to aerated solutions. The samples were tested in aerated sodium chloride 
solutions and aerated hydrochloric acid solutions. These studies will be compared with similar studies in deaerated solutions described in section 6.4 to understand the effect of dissolved oxygen

A SEM image of an intact coated-aluminum sample is shown in Figure 6-8. From this image, the interior and exterior coatings covering the aluminum can be seen. As will be shown later, the difference between intact and damaged coatings is very apparent in the SEM images.

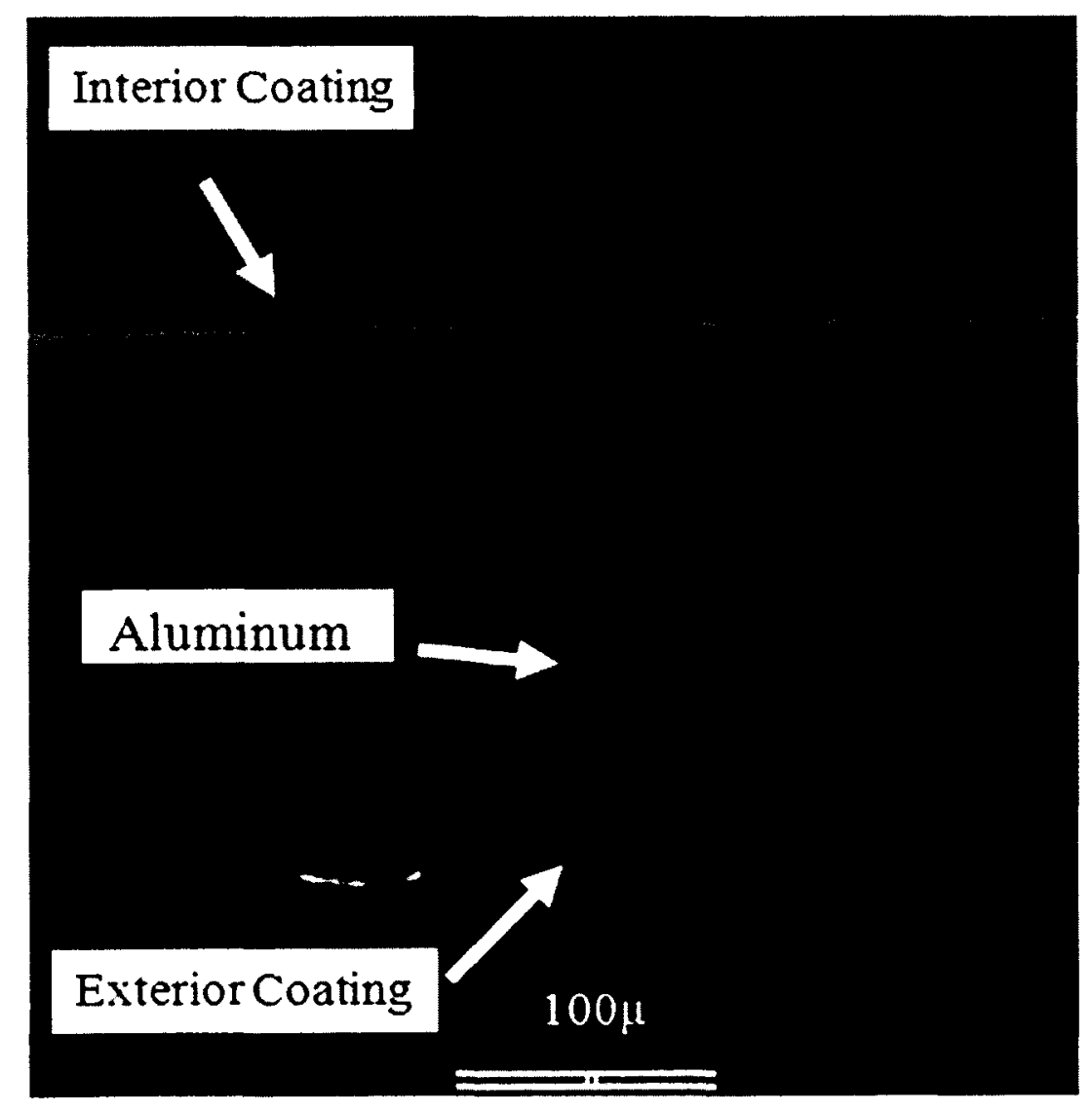

Figure 6-8 SEM image of an intact coated-aluminum sample. 


\subsubsection{Neutral Solutions}

\subsubsection{1. $1 \mathrm{M} \mathrm{NaCl}(\mathrm{pH} \mathrm{8})$}

Samples exposed to this solution showed active corrosion at pits and sometimes relatively large areas of complete dissolution of the aluminum.

A sample was placed in the horizontal cell with $1 \mathrm{M} \mathrm{NaCl}$ as the electrolyte and EIS data were collected using Gamry 1. Figure 6-9 shows that at 0 hours, the impedance of this sample is approximately $10^{7} \Omega$ at $0.01 \mathrm{~Hz}$, indicating a poorly protective coating likely containing a small natural flaw, not visibly obvious.

After 1 hour of exposure, a dramatic change is observed in the impedance plot: the impedance increased to $>10^{10} \Omega$ at $0.01 \mathrm{~Hz}$, and is purely capacitive. Surprisingly, this indicates a flaw-free, protective coating. The most probable cause for this occurrence is diffusion of the electrolyte through the existing flaw(s), leading to an oxide layer forming and blocking the conductive path. The surface is completely passivated, supporting the discussion of Figure 6-5. It seems, however, that in the case of this small flaw, passivation is much better than with the large scratch (section 6.2). This electrolyte has a $\mathrm{pH}$ of 8 which explains the stability of the aluminum oxide formed (see section 3.3.1).

The same observations can be seen in the phase-angle plot, Figure 6-10. At 0 hours, the phase plot starts off capacitive with capacitive-to-resistive transition observed at a frequency of $1000 \mathrm{~Hz}$ (also observed in Figure 6-9). Also, at the low end of the frequency spectrum, spikes were observed indicating some chemical reactions switching on and off at the metal-electrolyte interface. After 1 hour, the phase plot demonstrates a purely capacitive curve, indicated by a phase angle of $-90^{\circ}$ across the frequency spectrum. It is also important to note that the spikes no longer exist when the 
conductive paths are blocked. Hence, suggesting that spikes in the Bode plot are indeed indicative of random chemical reactions occurring at the surface of the metal substrate.

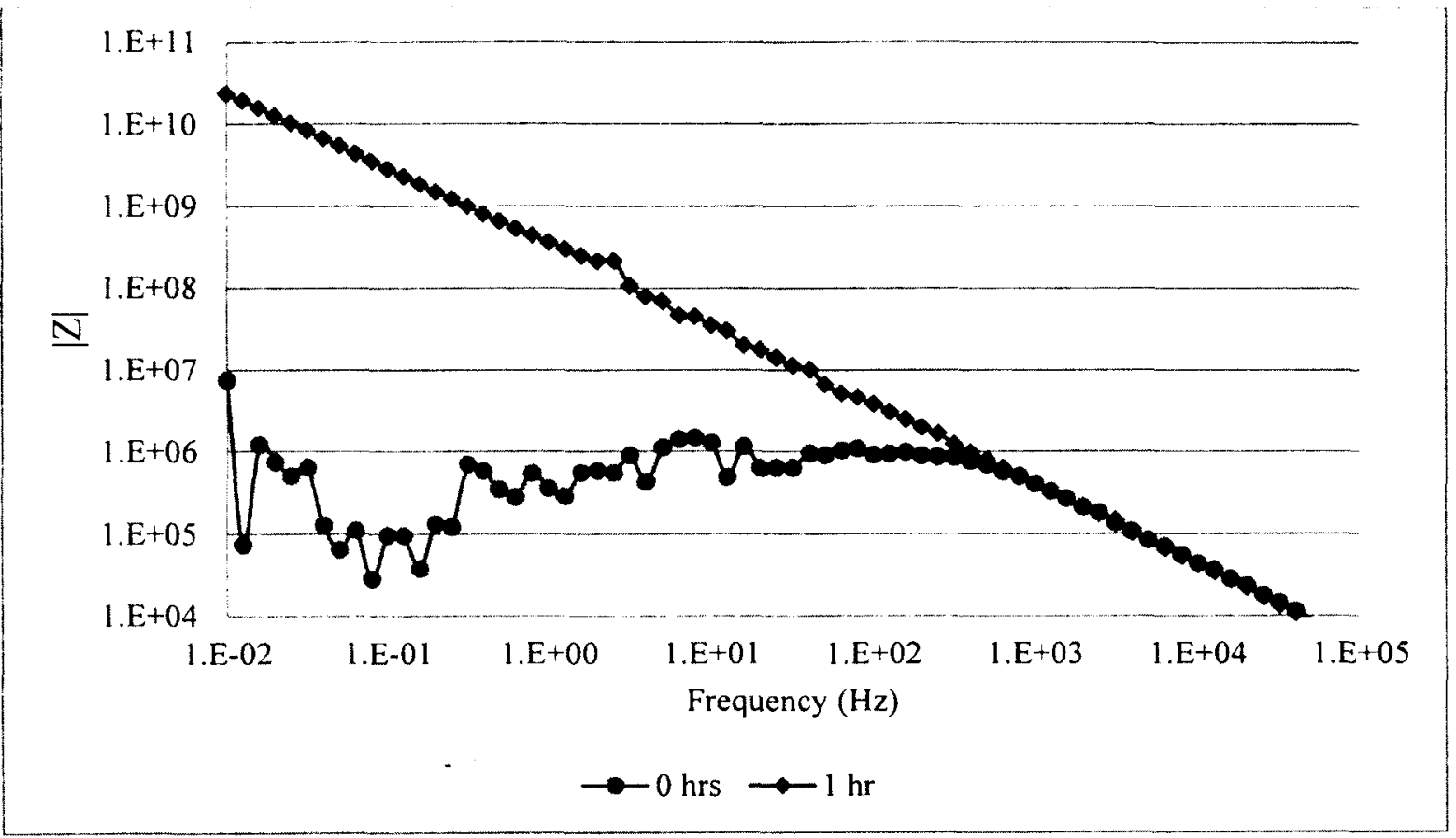

Figure 6-9 Impedance ( $\Omega$ ) measurements using Gamry $1\left(\mathrm{HC}, 1.0 \pm 0.1 \mathrm{~cm}^{2}\right)$ for coated aluminum sample in $1 \mathrm{M} \mathrm{NaCl}(\mathrm{pH} \mathrm{8})$. Note the passivation occurring that leads to an increase in the impedance. 


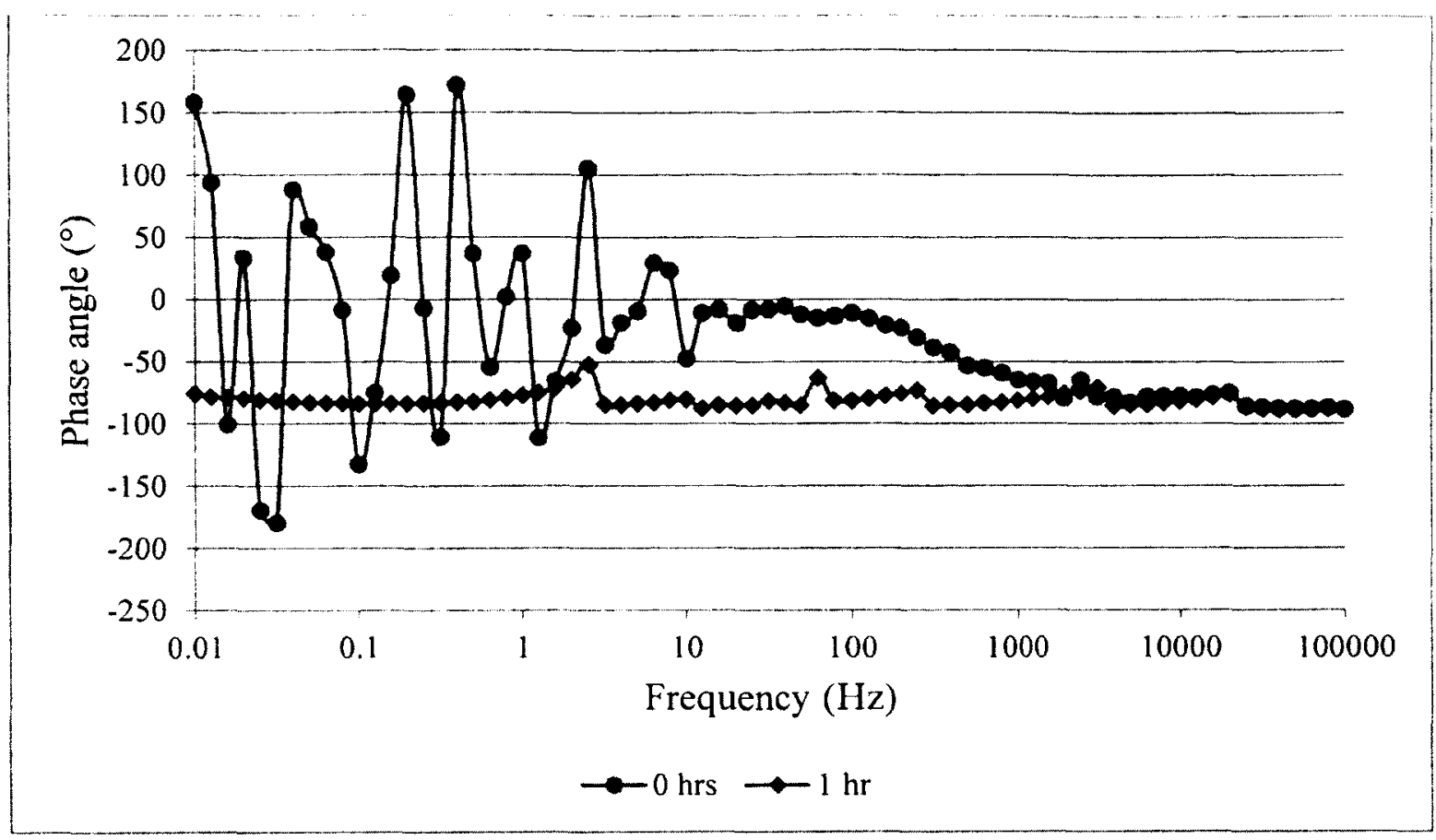

Figure 6-10 Phase angle measurements using Gamry $1\left(\mathrm{HC}, 1.0 \pm 0.1 \mathrm{~cm}^{2}\right)$ for coated aluminum sample in $1 \mathrm{M} \mathrm{NaCl}$ (pH 8). Note the disappearance of spikes and the purely capacitive plot after 1 hour of exposure.

Upon further exposure, as seen in Figure 6-11 and Figure 6-12, spiking starts to appear in both the impedance and phase plots after 79 hours. Shortly after, at 81 hours, a drop is observed in the impedance plot; $>10^{10} \Omega$ down to $<10^{9} \Omega$ at $0.01 \mathrm{~Hz}$, indicating a breach in the coating. By looking at the phase plot, the purely capacitive curve at 79 hours, is now capacitive-resistive at 81 hours. This confirms that the coating had been penetrated and the solution is reaching the aluminum substrate and some chemical reaction is taking place.

The impedance is then observed to rise again at 84 hours, indicating some repassivation occurring in the sample. It is also supported by a capacitive phase plot (at 84 hours). Two hours later, 86 hours of exposure, a large drop is noted in the impedance plot: down to $10^{6} \Omega$ at $0.01 \mathrm{~Hz}$, with a more resistive phase plot. The changes in 
impedance and phase angles are indications of intermittent passivation occurring on the metal surface. The impedance rises and the phase plots are more capacitive due to oxide forming on the metal surface. The oxide is not completely stable at $\mathrm{pH} 8$ (explained in section 3.3.1), it eventually breaks down causing the drop in impedance and the resistive values for the phase angles.

The phase and impedance plots show that the capacitive to resistive transition frequency is, as expected, increasing, which is consisting with an actively corroding sample.

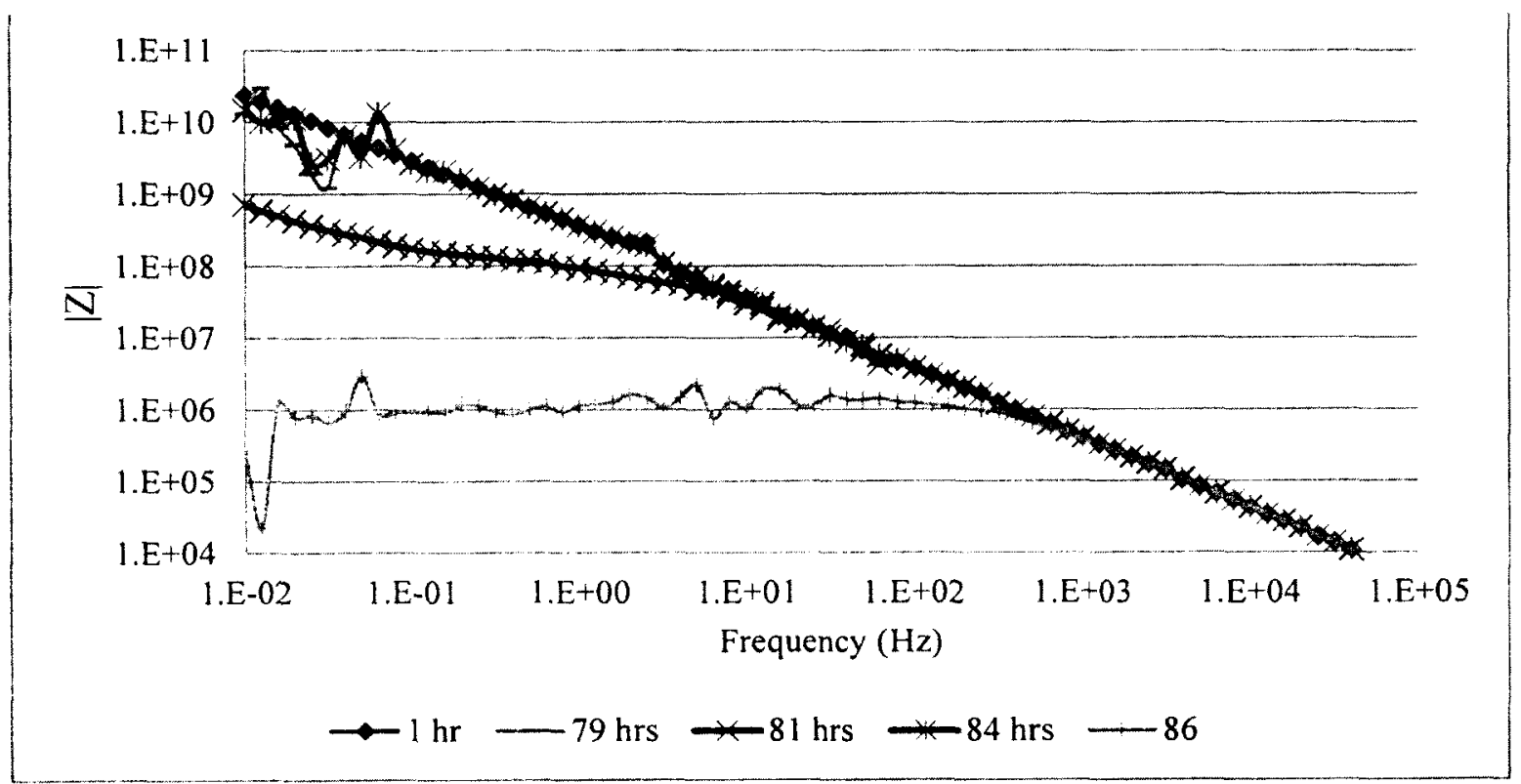

Figure 6-11 Impedance ( $\Omega$ ) measurements using Gamry $1\left(\mathrm{HC}, 1.0 \pm 0.1 \mathrm{~cm}^{2}\right)$ for coated aluminum sample in $1 \mathrm{M} \mathrm{NaCl}(\mathrm{pH} \mathrm{8})$. Note the rising and falling impedance indicating some intermittent passivation occurring on the surface of the metal substrate and intermittent blocking of the conductive paths. 


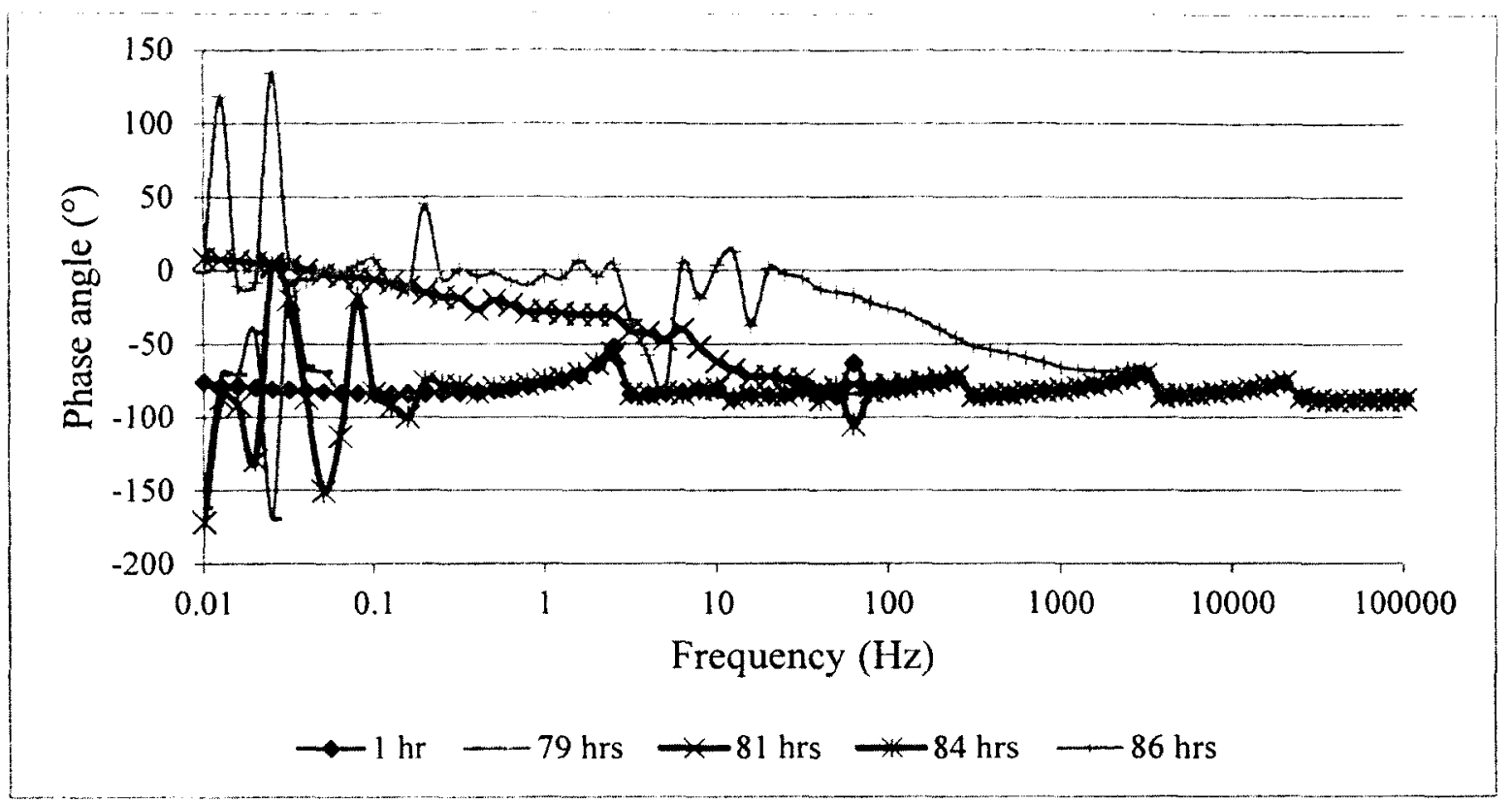

Figure 6-12 Phase angle measurements using Gamry $1\left(\mathrm{HC}, 1.0 \pm 0.1 \mathrm{~cm}^{2}\right)$ for coated aluminum sample in $1 \mathrm{M} \mathrm{NaCl}$. Note the intermittent passivation occurring on the surface of the metal indicated by changes in the phase angles from capacitive to resistive, and back to capacitive.

Figure 6-13 and Figure 6-14 demonstrate the remainder of the EIS data collected for this sample. The impedance keeps rising and falling, with an overall trend to decrease. The capacitive-to-resistive transition frequency is also observed to be increasing with prolonged exposure.

By examining the EIS data, a trend was observed in the impedance and phase plots. With exposure, spikes start to appear and increase in magnitude, and at some point a drop is observed in the impedance plot. At the new impedance, the spikes then start to shrink and after some time, a smooth curve is observed. This is then usually followed by an increase in the impedance. This shows that spiking is an indication of electrolyte penetrating through the coating and reaching the metal substrate. The increased chemical activity leads to current fluctuations leading to these spikes. Once the oxide is also breached and pits start to form on the metal surface, the impedance drops. 
Aluminum oxide is somewhat stable at this $\mathrm{pH}$. Oxide forming at the pitting sites explains the decrease in the magnitude of the spikes. If the actively corroding sites are all blocked by the aluminum oxide, the impedance is seen to rise: passivation. This can be seen in Figure 6-13; the impedance at 151 hours is greater than at 150 hours, and similarly the impedance at 161 hours is greater than at 160 hours.

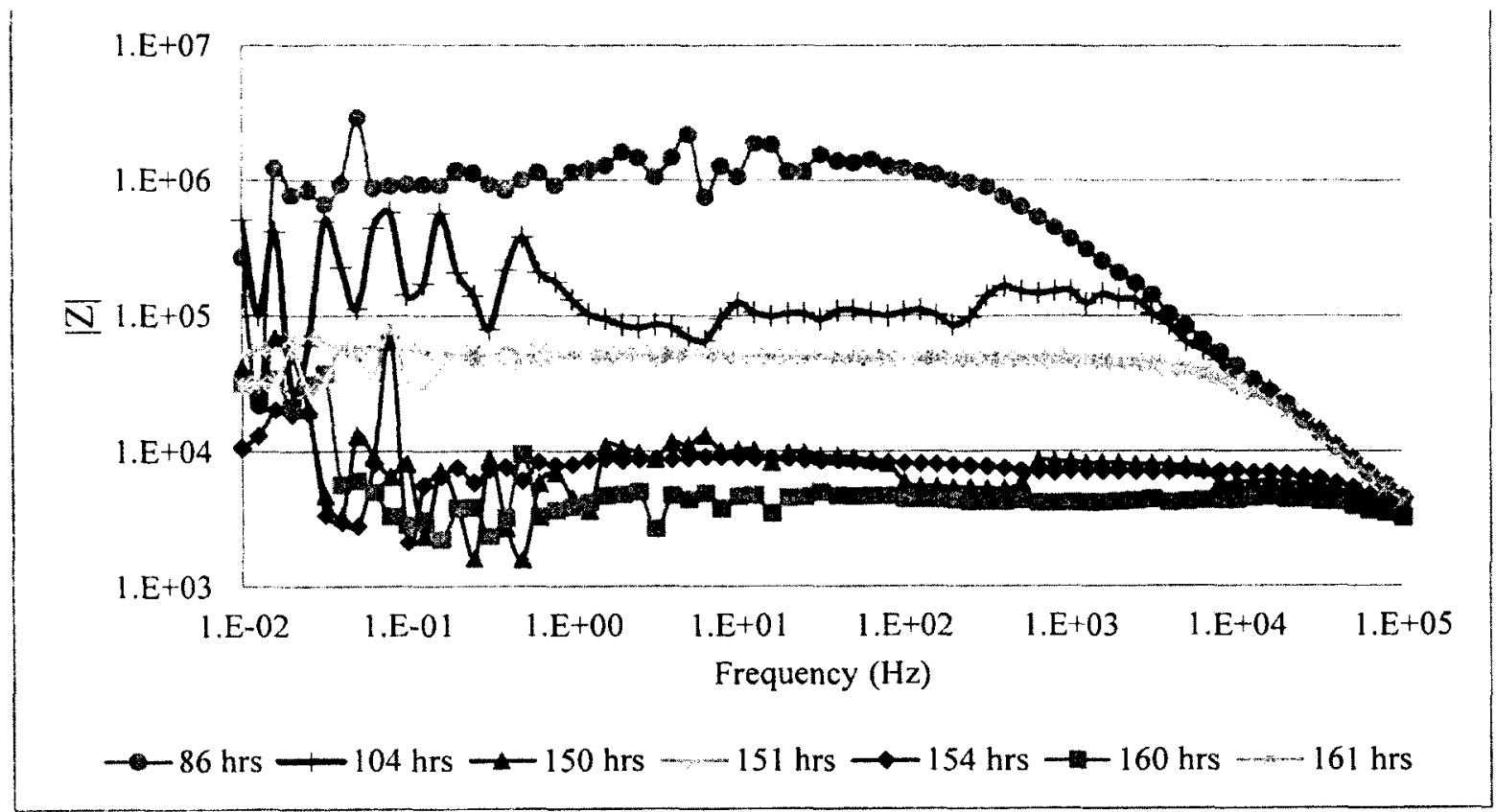

Figure 6-13 Impedance $(\Omega)$ measurements using Gamry $1\left(\mathrm{HC}, 1.0 \pm 0.1 \mathrm{~cm}^{2}\right)$ for coated aluminum sample in $1 \mathrm{M} \mathrm{NaCl}(\mathrm{pH} 8)$. Note the rise and fall in impedance with exposure and the increasing capacitive-to-resistive transition frequency. 


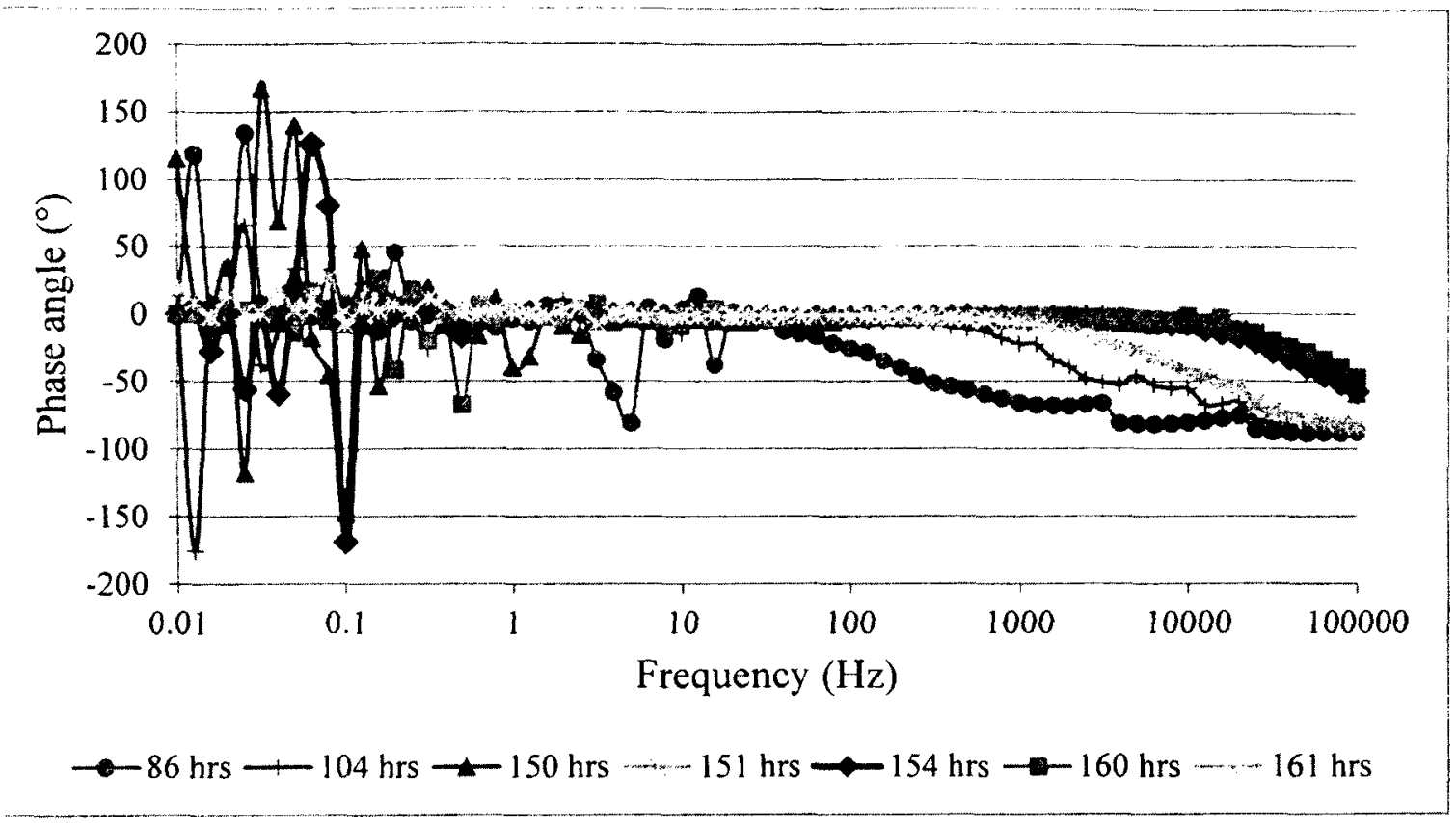

Figure 6-14 Phase angle measurements using Gamry $1\left(\mathrm{HC}, 1.0 \pm 0.1 \mathrm{~cm}^{2}\right)$ for coated aluminum sample in $1 \mathrm{M} \mathrm{NaCl} \mathrm{(pH} \mathrm{8).} \mathrm{Note} \mathrm{the} \mathrm{increasing}$ capacitive-to-resistive transition frequency with exposure time.

Figure 6-15 was captured while the experiment was running. A white cloud was observed near the sample, which is attributed to aluminum oxide. As mentioned above, the samples repassivate for a while but then it appears that the oxide becomes permeable, some oxide is ejected, and the impedance drops. This interpretation is consistent with the appearance of this oxide cloud. In some experiments oxide trees were observed to grow out of pits. 


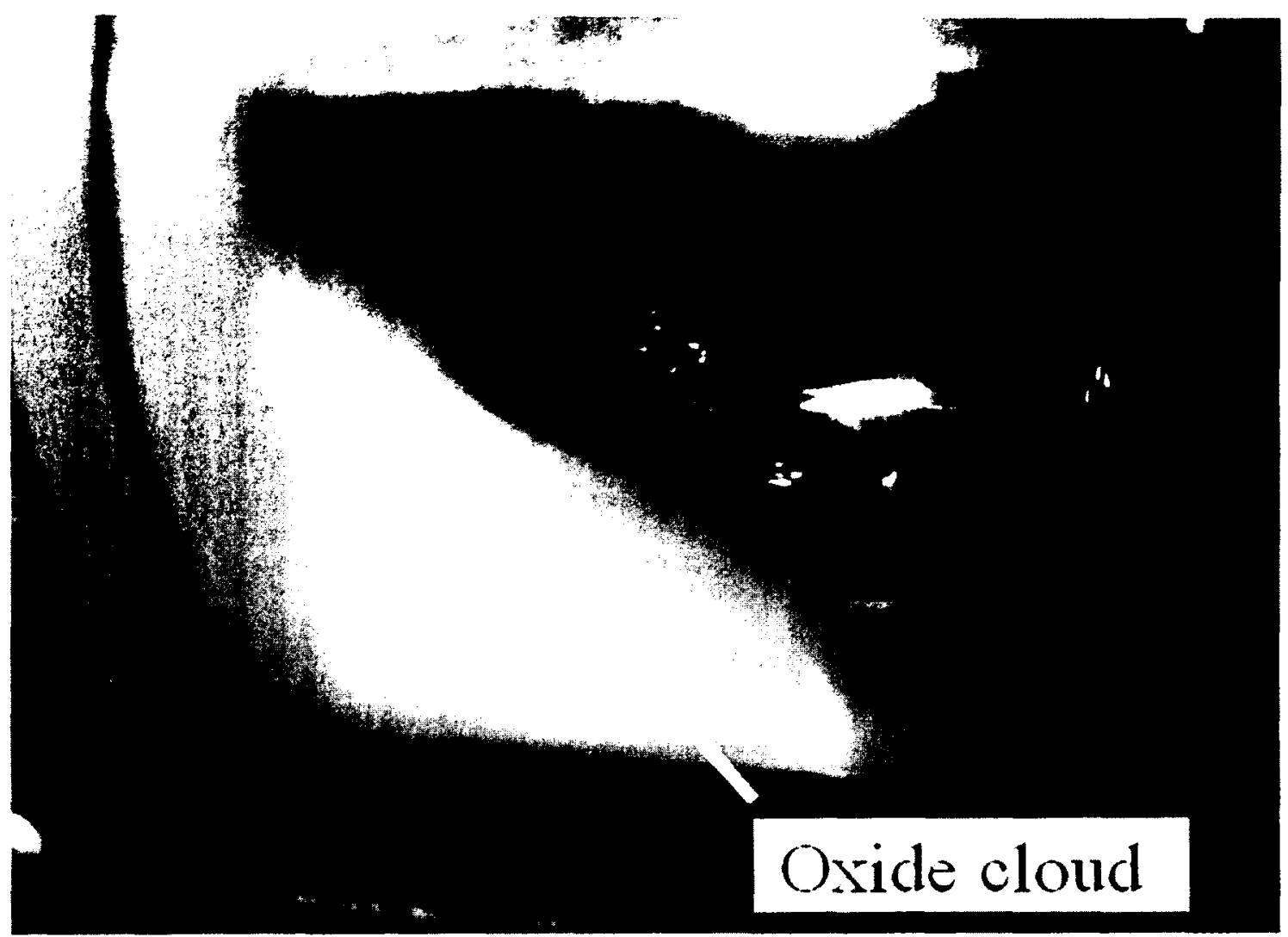

Figure 6-15 Oxide cloud observed in the horizontal cell (Figure 4-2) during the immersion of a coated aluminum sample in $1 \mathrm{M}$ NaCl (pH 8).

During the experiment, bubbles were observed close to the sample as shown in Figure 6-16. These bubbles are believed to be hydrogen due to the cathodic process (equation 3-7) occuring in the corroding coated aluminum sample. Another explanation for the apparent intermittent rising and falling of the impedance could be hydrogen bubbles forming in pits and expelling solution and thus causing the impedance to rise. This is considered unlikely because corrosion occurred over such relatively large areas, but further investigation is required. 


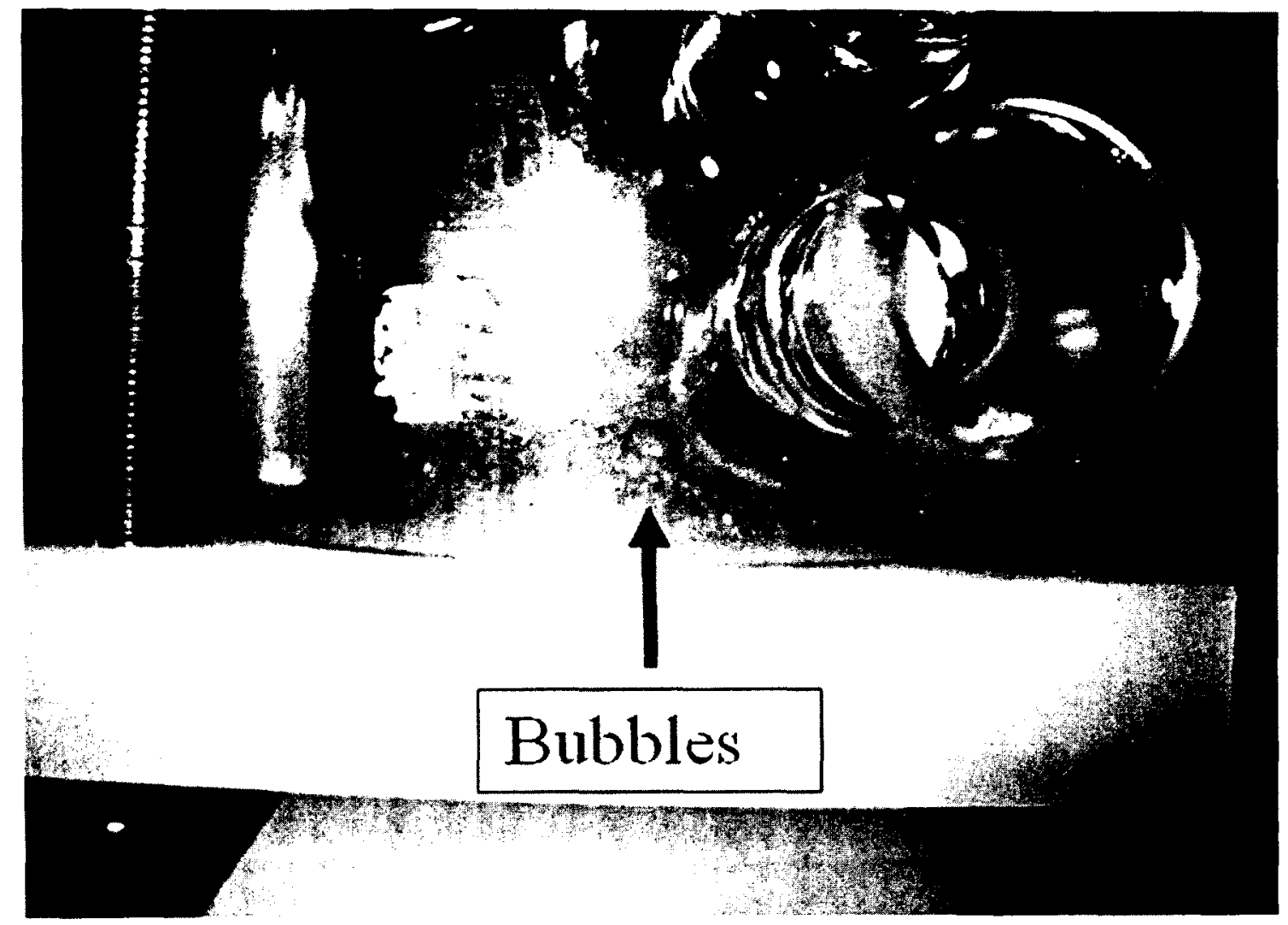

Figure 6-16 Bubbles on the sample are observed in the horizontal cell (Figure 4-2) during the exposure of a coated aluminum sample in $1 \mathrm{M} \mathrm{NaCl}(\mathrm{pH} \mathrm{8})$.

Figure 6-17 shows the final condition of the coated aluminum sample. Several pits (dark spots) and through holes (white spots) are observed on the sample. This sample failed after a total exposure time of 161 hours approximately. 


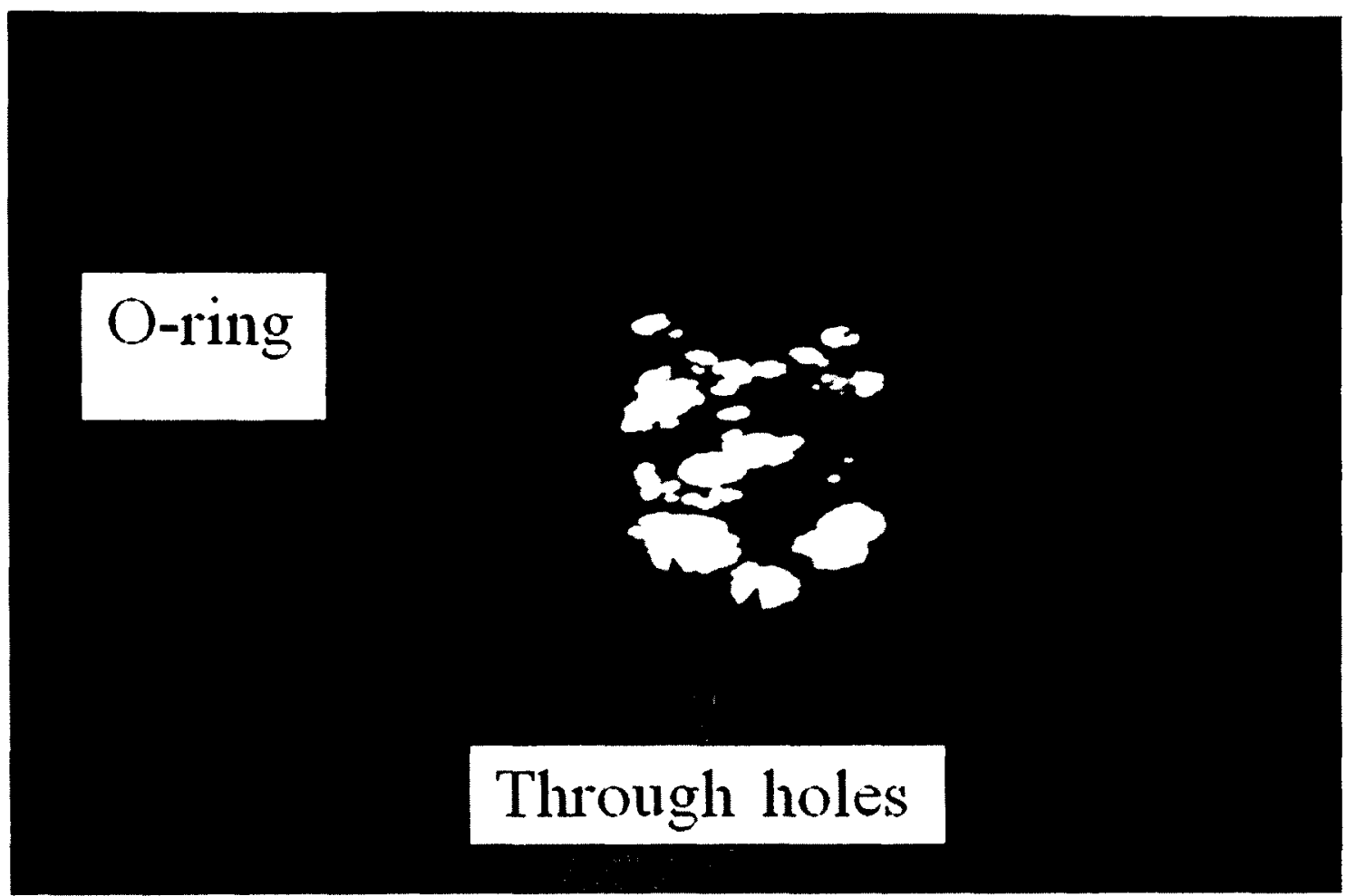

Figure 6-17 Condition of a coated aluminum sample after exposure to $1 \mathrm{M} \mathrm{NaCl}$ (pH 8 ) for $161 \mathrm{hrs}$ in the horizontal cell (Figure 4-2).

\subsubsection{0.1 $\mathrm{M} \mathrm{NaCl}(\mathrm{pH} \mathrm{8})$}

An intact coating was exposed to $0.1 \mathrm{M} \mathrm{NaCl}(\mathrm{pH} \mathrm{8).} \mathrm{The} \mathrm{Bode} \mathrm{and} \mathrm{phase} \mathrm{plots} \mathrm{are}$ shown in Figure 6-18 and Figure 6-19. As explained in section 5.2.3, Solartron 1 cannot measure impedances higher than $10^{8} \Omega$. It is working at its limit, which explains the spiking occurring in the impedance and phase-angle plots (Figure 6-18 and Figure 6-19).

The results for this sample are similar to the sample in $1 \mathrm{M} \mathrm{NaCl}$, but the data shows a sudden change that was not seen previously. Looking at Figure 6-18, it can be seen that at 7 hours, the impedance 'suddenly' increases from $10^{6} \Omega$ to $10^{8} \Omega$ at $0.01 \mathrm{~Hz}$, followed by increased impedance across the whole frequency spectrum obtained at 8 hours. This sudden sustained increase in impedance suggests that passivation is occurring in neutral solutions. 


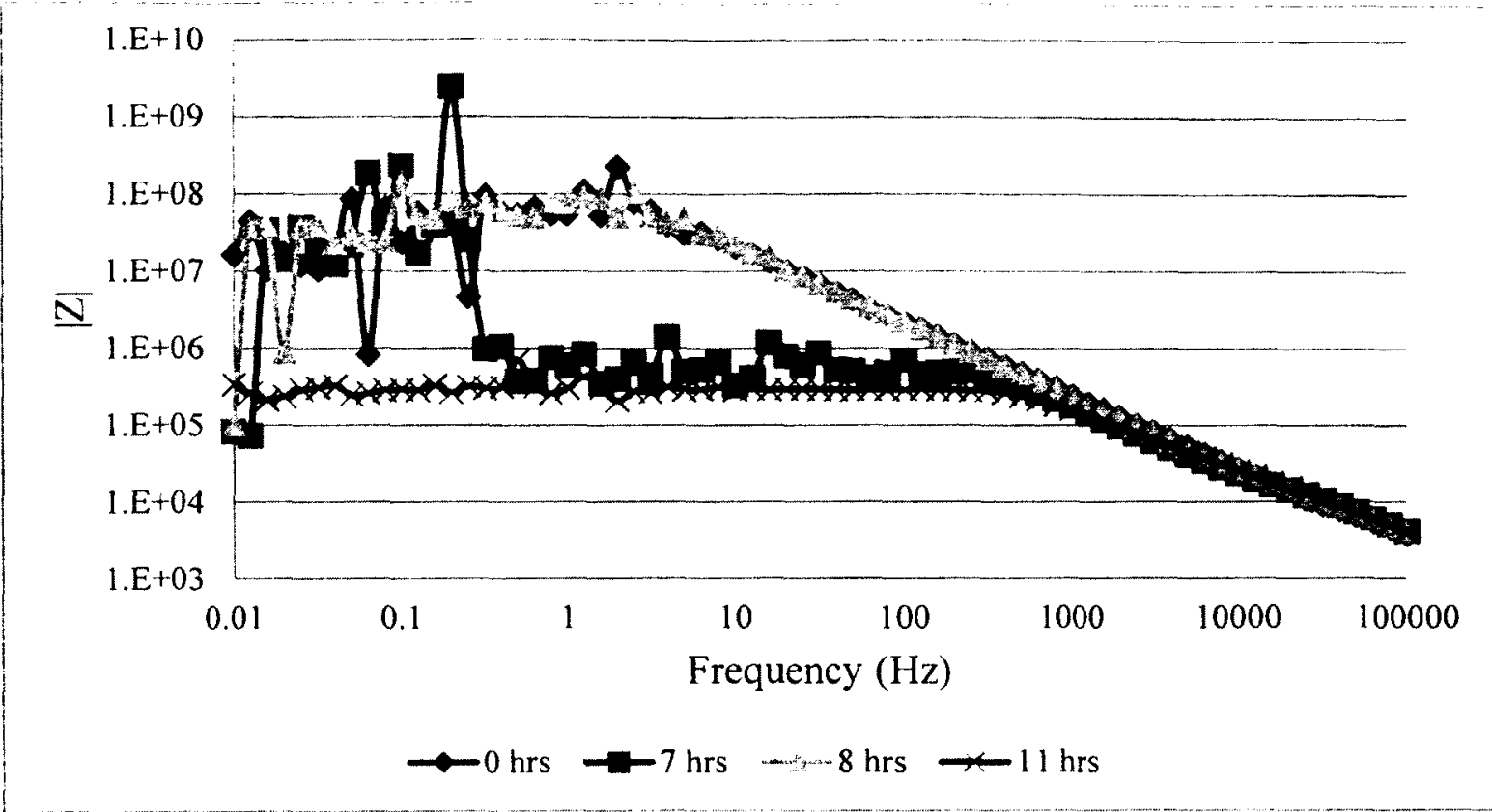

Figure 6-18 Impedance $(\Omega)$ measurements using Solartron $1\left(\mathrm{VC}, 34.0 \pm 0.5 \mathrm{~cm}^{2}\right)$ for a coated aluminum sample in $0.1 \mathrm{M} \mathrm{NaCl}$ ( $\mathrm{pH}$ 8). Note the sudden increase in impedance at $7 \mathrm{hrs}$, indicating passivation.

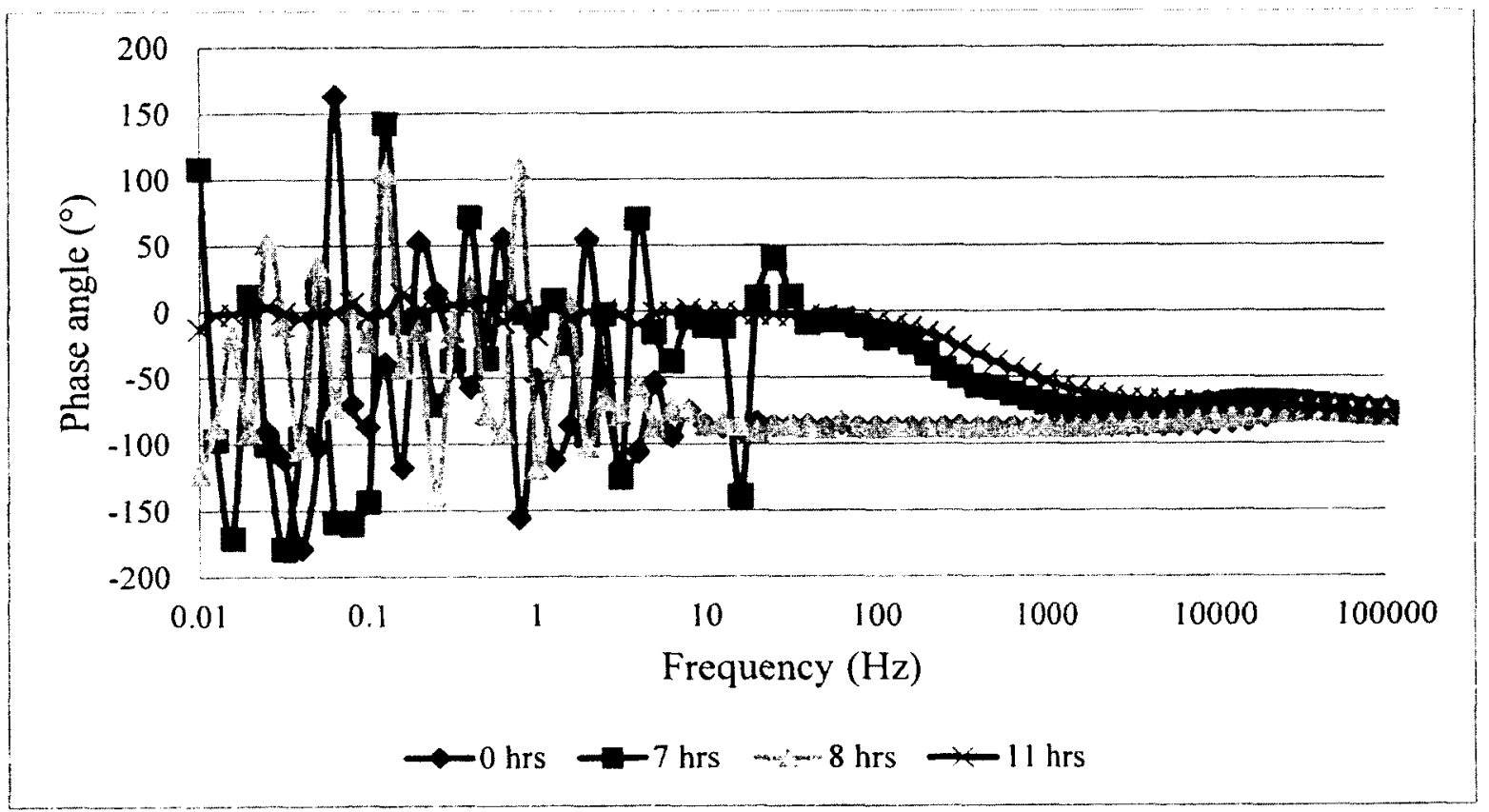

Figure 6-19 Phase angle measurements using Solartron $1\left(\mathrm{VC}, 34.0 \pm 0.5 \mathrm{~cm}^{2}\right)$ for a coated aluminum sample in $0.1 \mathrm{M} \mathrm{NaCl}(\mathrm{pH} \mathrm{8})$. 
Figure 6-20 and Figure 6-21 show more of the EIS data collected for the sample in $0.1 \mathrm{M} \mathrm{NaCl}$. Looking at Figure 6-20, the intermittent passivation, once again, is evident at 16 and 26 hours. It is important to note, that with prolonged exposure and the degradation of the sample, the spiking occurring in both the impedance and phase plots, is decreasing in magnitude. Also, the capacitive to resistive transition frequency increases with sample degradation, as observed before.

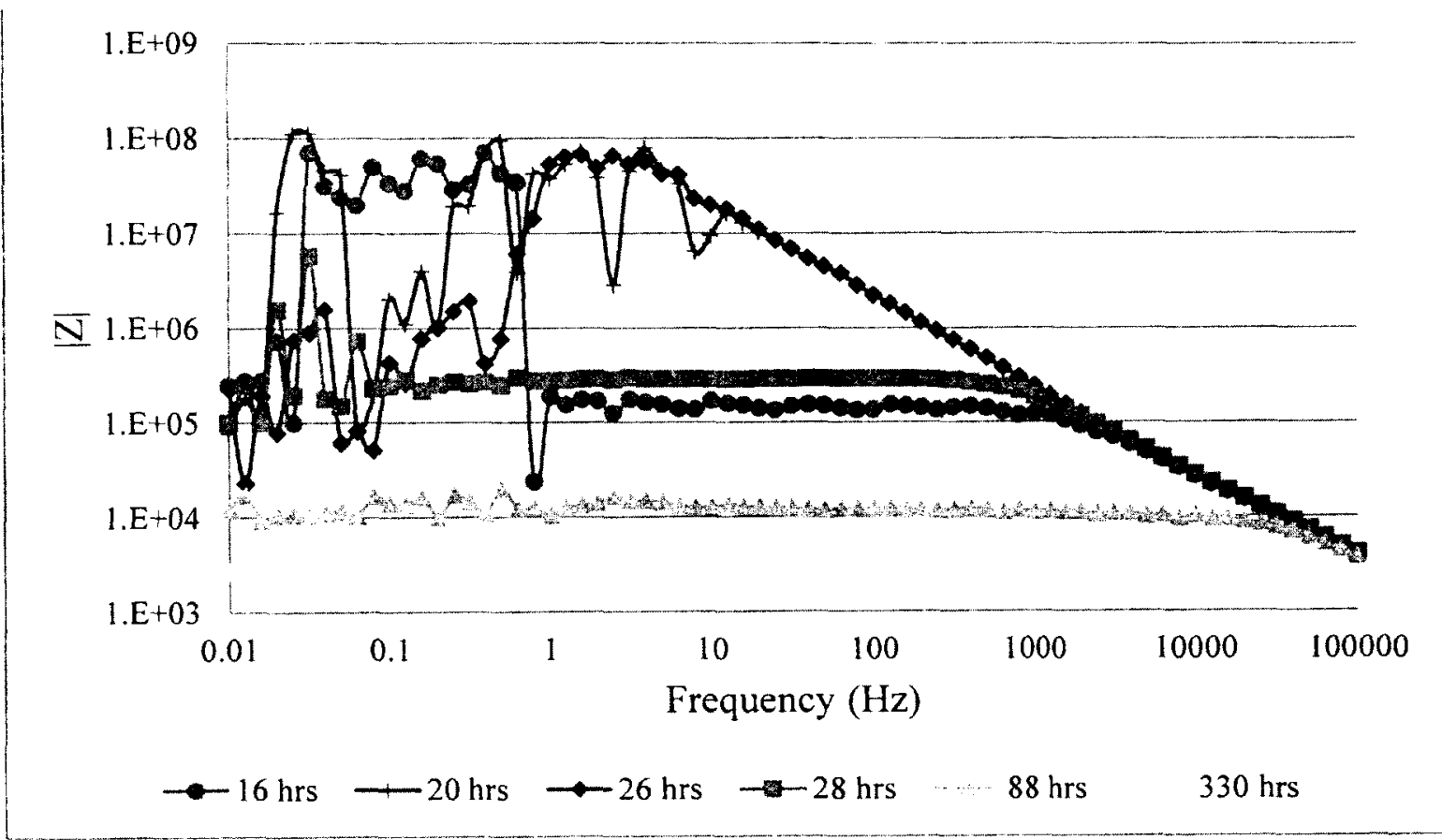

Figure 6-20 Impedance $(\Omega)$ measurements using Solartron $1\left(\mathrm{VC}, 34.0 \pm 0.5 \mathrm{~cm}^{2}\right)$ for a coated aluminum sample in $0.1 \mathrm{M} \mathrm{NaCl}(\mathrm{pH} \mathrm{8})$. Note the sudden drop in impedance at $26 \mathrm{hrs}$ suggesting the opening of a conductive path in the coating. 


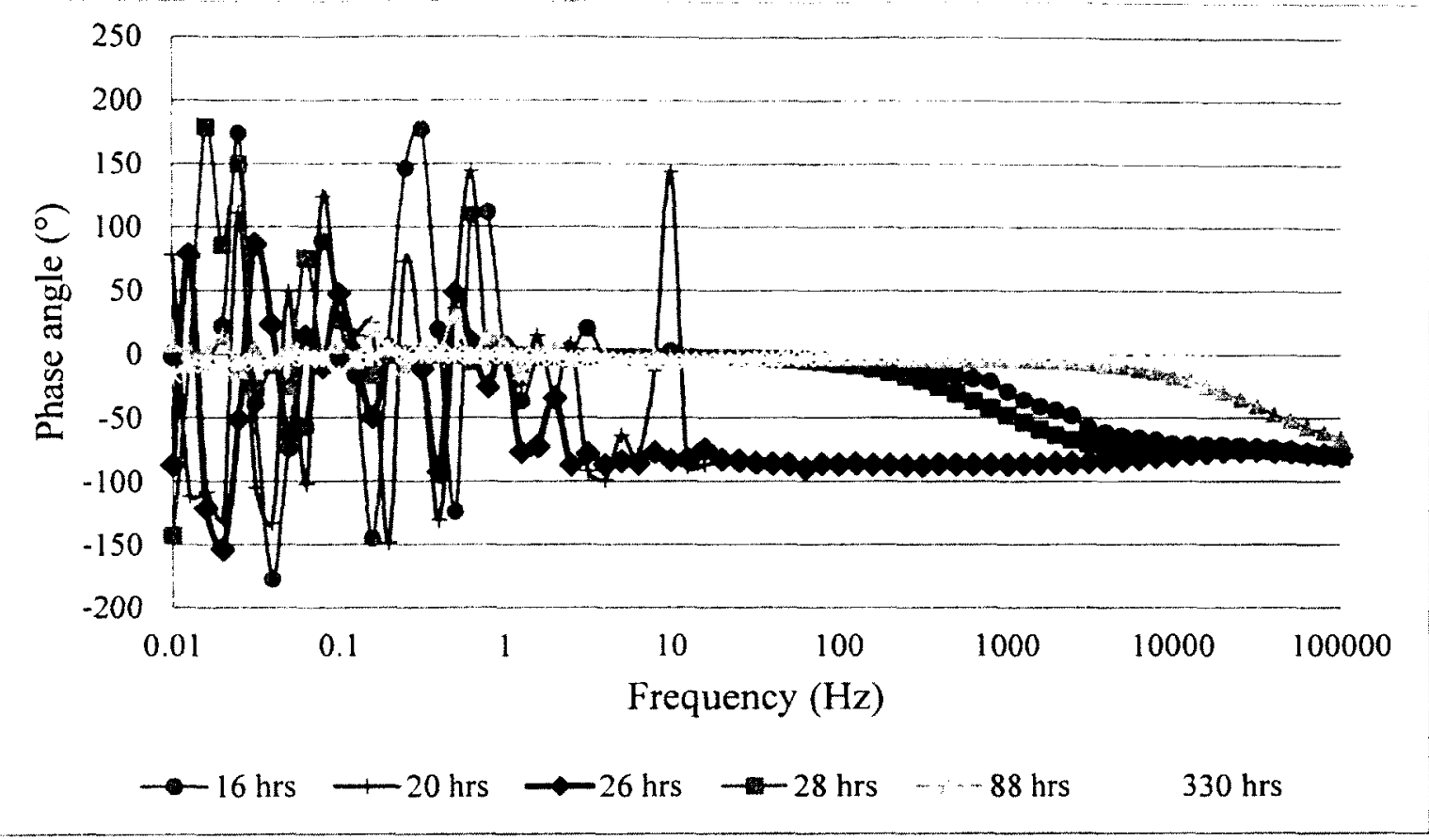

Figure 6-21 Phase angle measurements using Solartron $1\left(\mathrm{VC}, 34.0 \pm 0.5 \mathrm{~cm}^{2}\right)$ for a coated aluminum sample in $0.1 \mathrm{M} \mathrm{NaCl}$ (pH 8).

This experiment was performed in the vertical cell and it was possible to observe the sample surface. As with the experiment in $1 \mathrm{M} \mathrm{NaCl}$, bubbles occurred on the surface which corresponded to the pitting sites. The bubbles are undoubtedly hydrogen gas evolving due to the corrosion of the metal. Note that changes in the Bode plots were observed much earlier than any visible changes on the metal surface.

From the bubbling/pitting sites, a white solid was seen to evolve: aluminum oxide forming in the pits. The oxide is not particularly soluble at this $\mathrm{pH}$ level, but it is not adherent either. It does not form a physically stable passive film. Over time it is extruded from the corrosion sites. This is why it was observed floating in the solution as seen in Figure 6-22. This provides an explanation for the intermittent passivation occuring in the EIS data. During corrosion, an oxide film forms in the pitting sites, but this film is intermittently penetrated by solution and the oxide is intermittently extruded from the pit. 


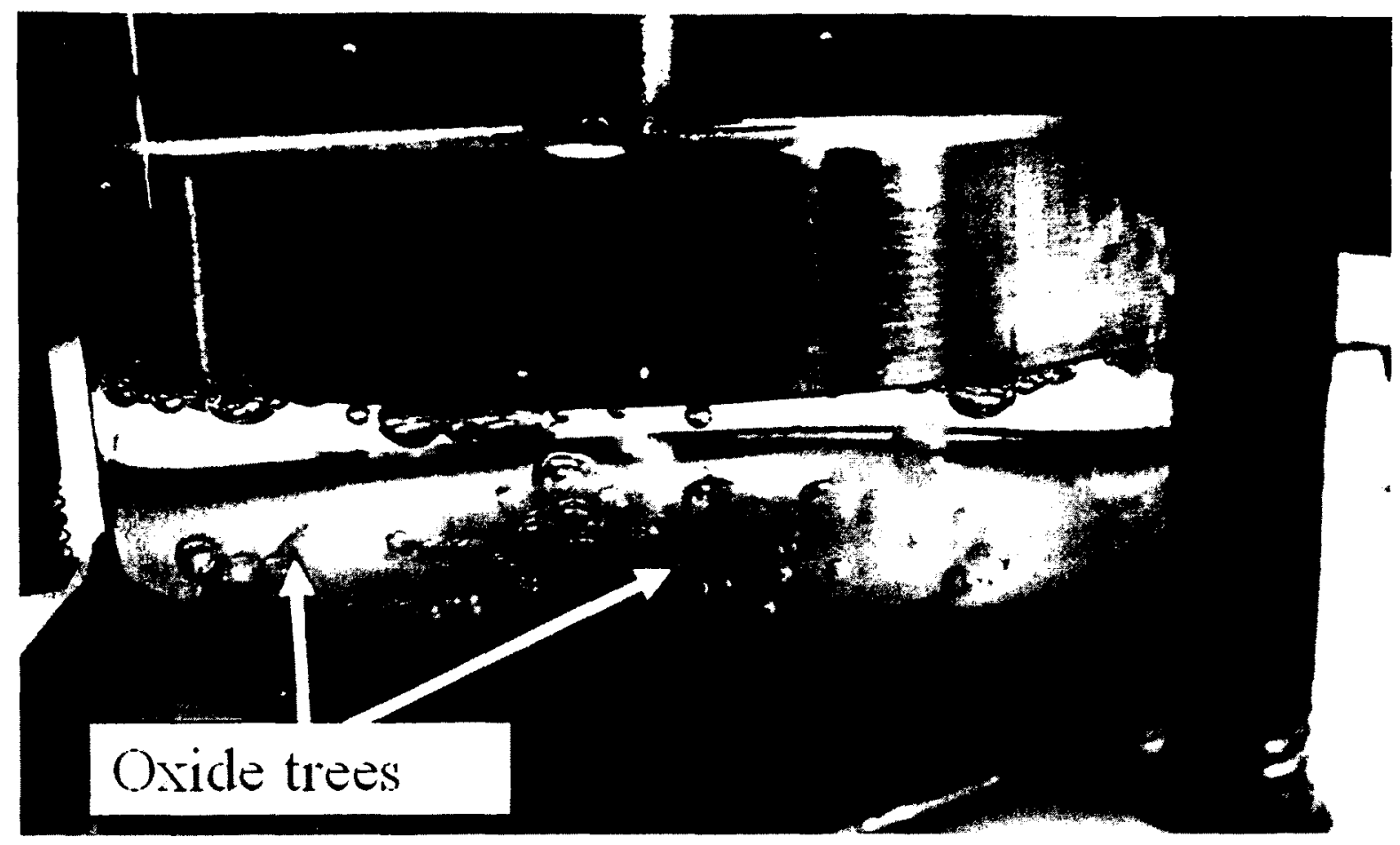

Figure 6-22 Oxide trees observed to emerge from pitting sites, in the vertical cell (Figure 4-1), during the immersion of a coated aluminum sample in 0.1 $\mathrm{M} \mathrm{NaCl}$ (pH 8). Some of the oxide was seen flaoting in the solution

After 330 hours of exposure, the impedance of the sample was between $10^{3} \Omega$ and $10^{4} \Omega$ at all frequencies, which is believed to be a measure of the solution resistance, demonstrating a conductive path and total penetration of the sample. This is also observed in the phase plot by a phase angle of $0^{\circ}$ across the frequency spectrum.

When the sample was removed, several pits and through-holes were observed in the surface as shown in Figure 6-23. This sample in $0.1 \mathrm{M} \mathrm{NaCl}$ failed after 330 hours, while the sample in $1 \mathrm{M} \mathrm{NaCl}$ failed after 161 hours. This is most likely due to the different aggressiveness of the solutions, and partly due to sample variability. 


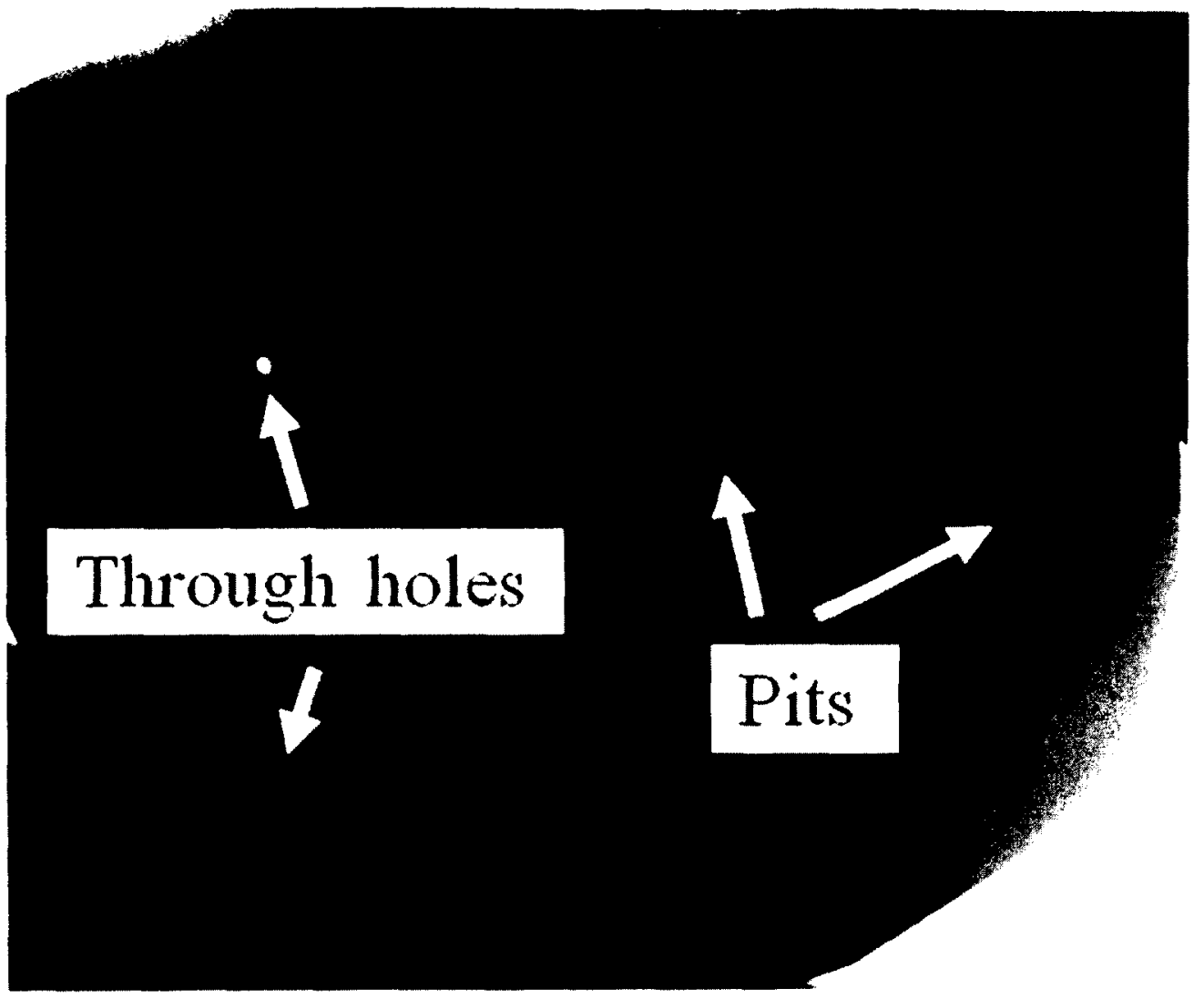

Figure 6-23 Condition of a coated aluminum sample after exposure to $0.1 \mathrm{M} \mathrm{NaCl}$ (pH 8) for 330 hours in the vertical cell (Figure 4-1). Pits and through holes were found on the surface of the sample.

Figure 6-24 shows a SEM image of a cross-section of a pitting site. Around the pit, the interior coating still exists, but in the pit, aluminum and some aluminum oxide are observed. It is likely that some aluminum oxide was lost when the cross-section was cut. 


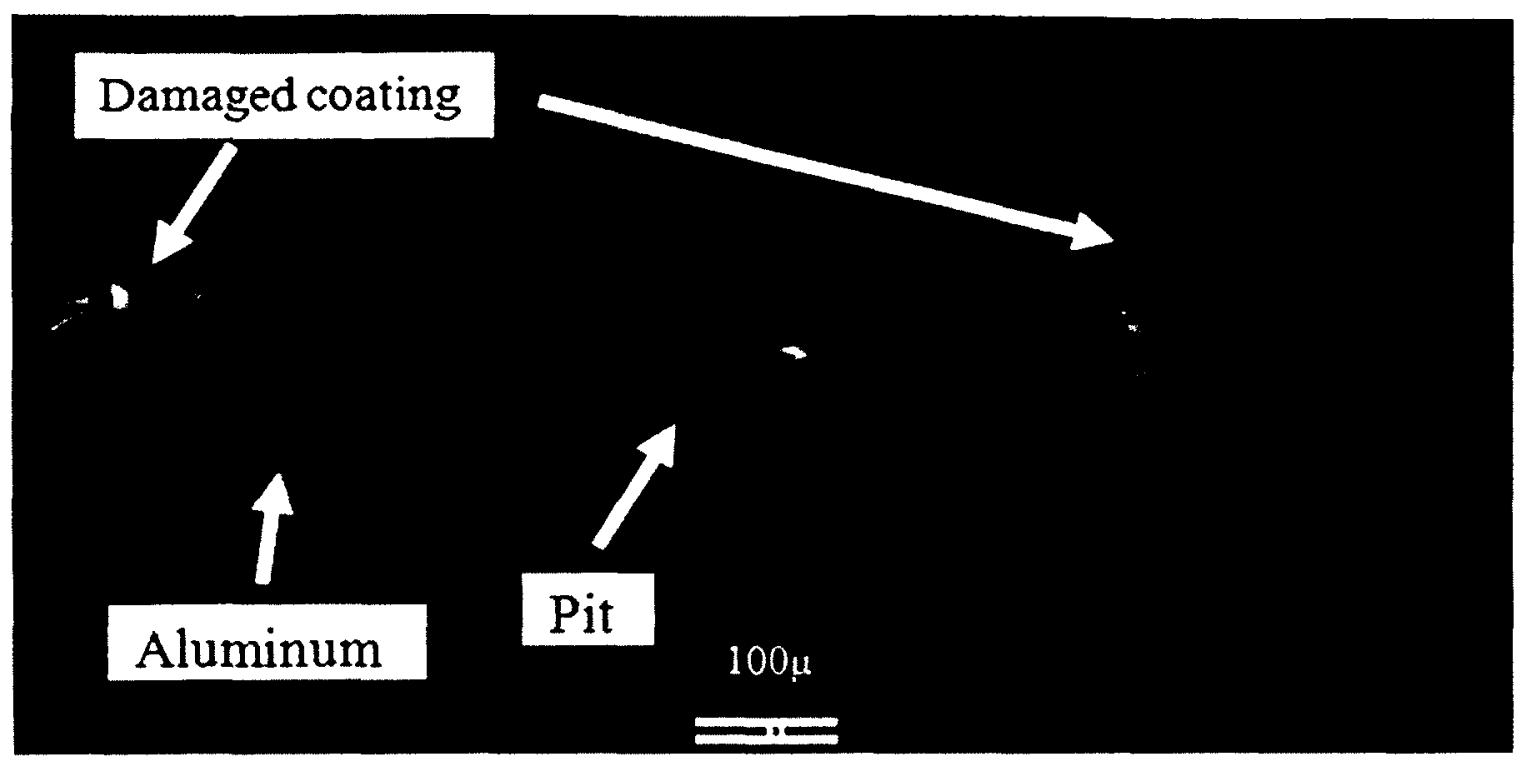

Figure 6-24 SEM image of a pit in the sample exposed to $0.1 \mathrm{M} \mathrm{NaCl}(\mathrm{pH} \mathrm{8})$.

The image in Figure 6-25 was taken at a higher angle and without cutting the sample. It shows the condition of the sample where it seems that a bubble was formed under the coating and had burst. Inside the cavity, aluminum is observed, but also aluminum oxide (corrosion product) is visible. This agrees with the observation of the white oxide clouds/trees forming in the pits.

The question arises again as to whether hydrogen accumulating under the coating can provide some passivation-like properties by ejecting solution and limiting the current path (see earlier discussion at the end of section 6.3.1.1). This explanation needs more investigation. 


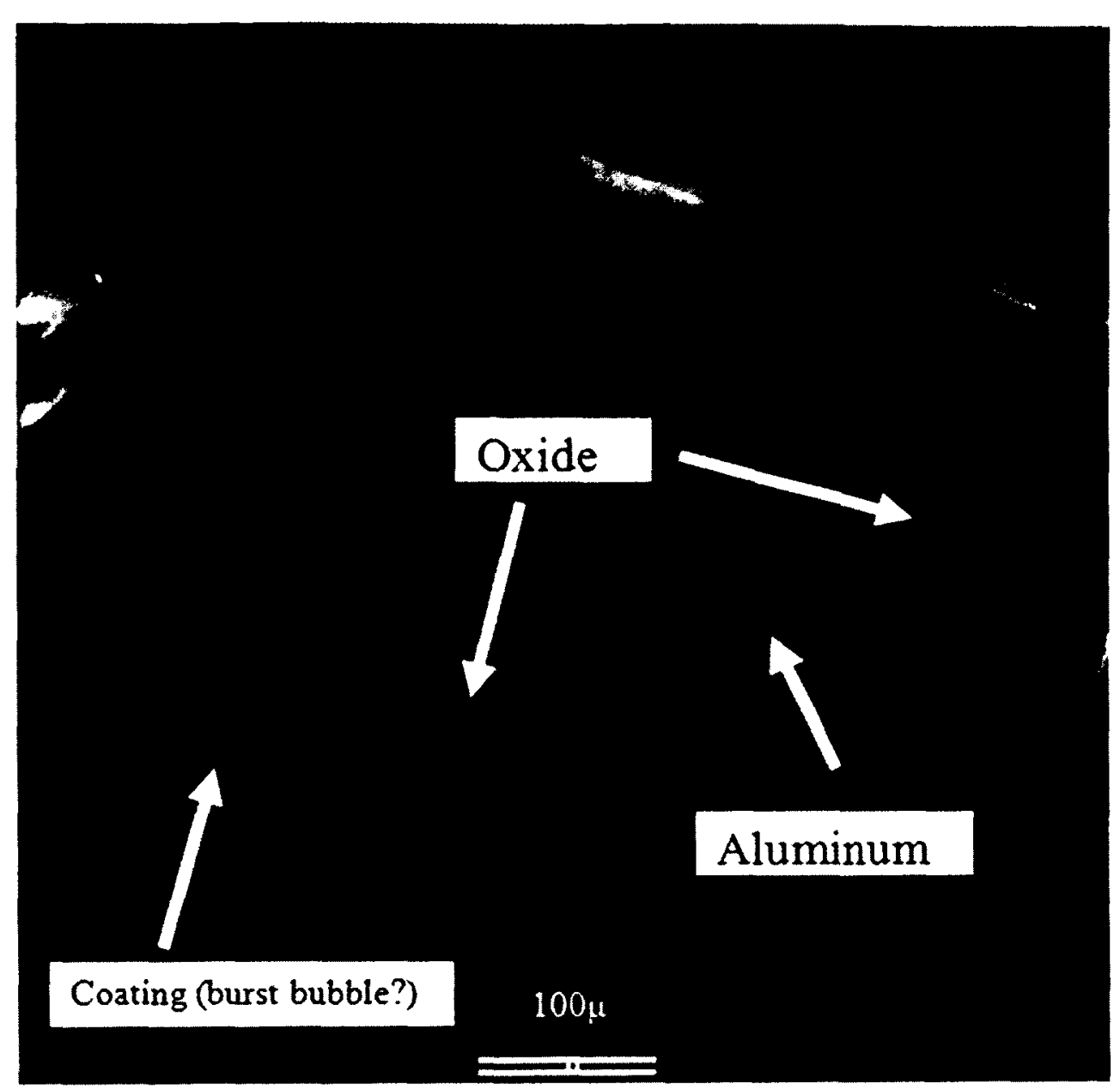

Figure 6-25 SEM image of a pit in the sample exposed to $0.1 \mathrm{M} \mathrm{NaCl}(\mathrm{pH} 8)$.

\subsubsection{Acidic Solutions}

\subsubsection{1. $1 \mathrm{M} \mathrm{HCl}(\mathrm{pH} \mathrm{0.1})$}

After performing experiments at a $\mathrm{pH}$ of $\sim 8$, experiments at the lower end of the $\mathrm{pH}$ scale were done. A sample was placed in a $1 \mathrm{M} \mathrm{HCl}$ solution ( $\mathrm{pH} 0.1$ ) and EIS data were collected (Figure 6-26). For approximately 850 hours, no changes in the impedance or phase plots were observed; it was purely capacitive compared with the samples in $\mathrm{NaCl}$ solutions at $\mathrm{pH} 8$, the coating seemed to be more resistant under these acidic conditions. 


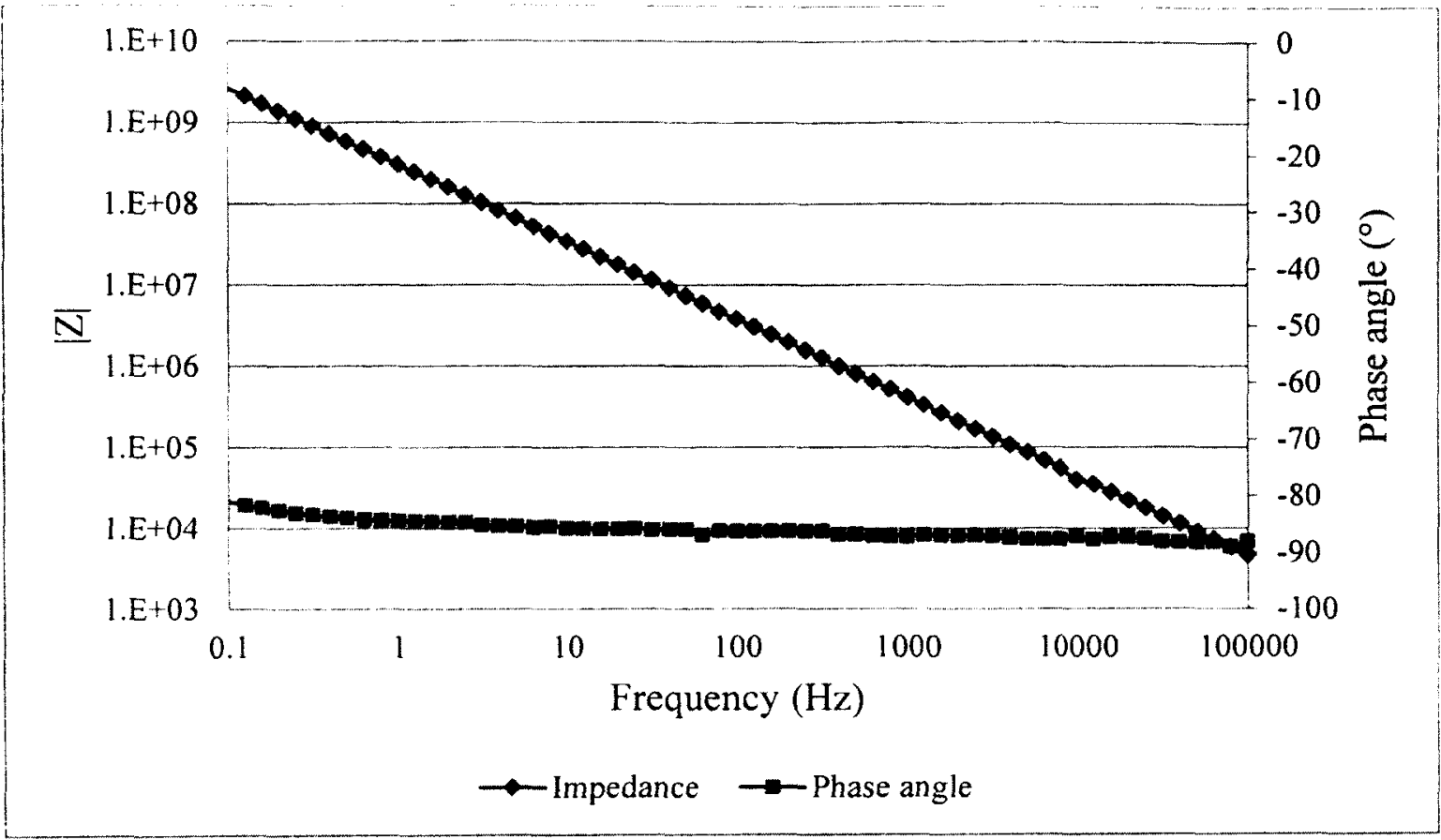

Figure 6-26 Bode plot (using Gamry 1) for sample in $1 \mathrm{M} \mathrm{HCl} \mathrm{(pH} \mathrm{0.1)} \mathrm{in} \mathrm{HC}$ $\left(1.0 \pm 0.1 \mathrm{~cm}^{2}\right)$. No changes were observed in the Bode plot for 850 hours.

Figure 6-27 shows the Nyquist plot for the same intact coating. As expected, from the literature [12], such samples should have an almost straight vertical line in a Nyquist plot, which, as an aside, could be used to differentiate between intact and flawed coatings. 


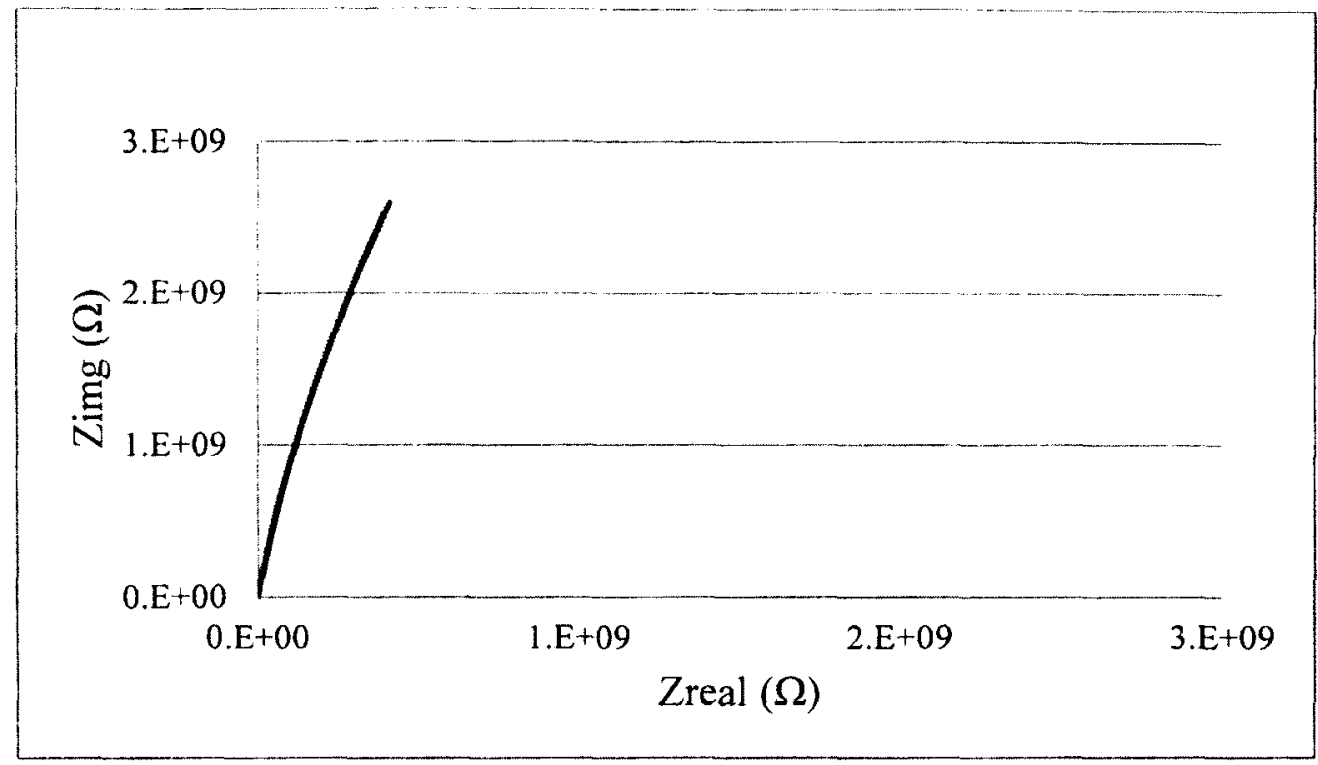

Figure 6-27 Nyquist plot (using Gamry 1) for sample in $1 \mathrm{M} \mathrm{HCl}(\mathrm{pH} \mathrm{0.1)}$ in $\mathrm{HC}$ $\left(1.0 \pm 0.1 \mathrm{~cm}^{2}\right)$.

The EIS data for a similar experiment are shown in Figure 6-28 and Figure 6-29; another sample in $1 \mathrm{M} \mathrm{HCl}(\mathrm{pH} 0.1)$, but now showing how the spectra vary with time. Only after 900 hours was a drop in the impedance observed, $10^{8} \Omega$ down to $10^{6} \Omega$ at $0.01 \mathrm{~Hz}$. Unlike the samples in $\mathrm{NaCl}$ solutions, no passivation was observed in the impedance plots; the impedance continuously dropped. No passivation was expected to occur at such a low $\mathrm{pH}$ level, because under such conditions, the oxide is unstable and soluble, and hence does not form; as discussed in section 3.3.1.

With continued exposure, the impedance continued to drop and the spikes, once again, continued to decrease in size. As with the samples in neutral solutions (section 6.3.1), the capacitive to resistive transition frequency increased. The sample was judged to have failed after a total of 1200 hours of exposure, which was 300 hours after the first drop in impedance at 900 hours. 
After the total penetration of the sample, the final impedance was $100 \Omega$ for all frequencies, which is the solution resistance. This is lower when compared to the $1 \mathrm{M}$ $\mathrm{NaCl}$ and $0.1 \mathrm{M} \mathrm{NaCl}$ solutions, because of the increased conductivity of $\mathrm{pH} 0.1 \mathrm{HCl}$ solution.

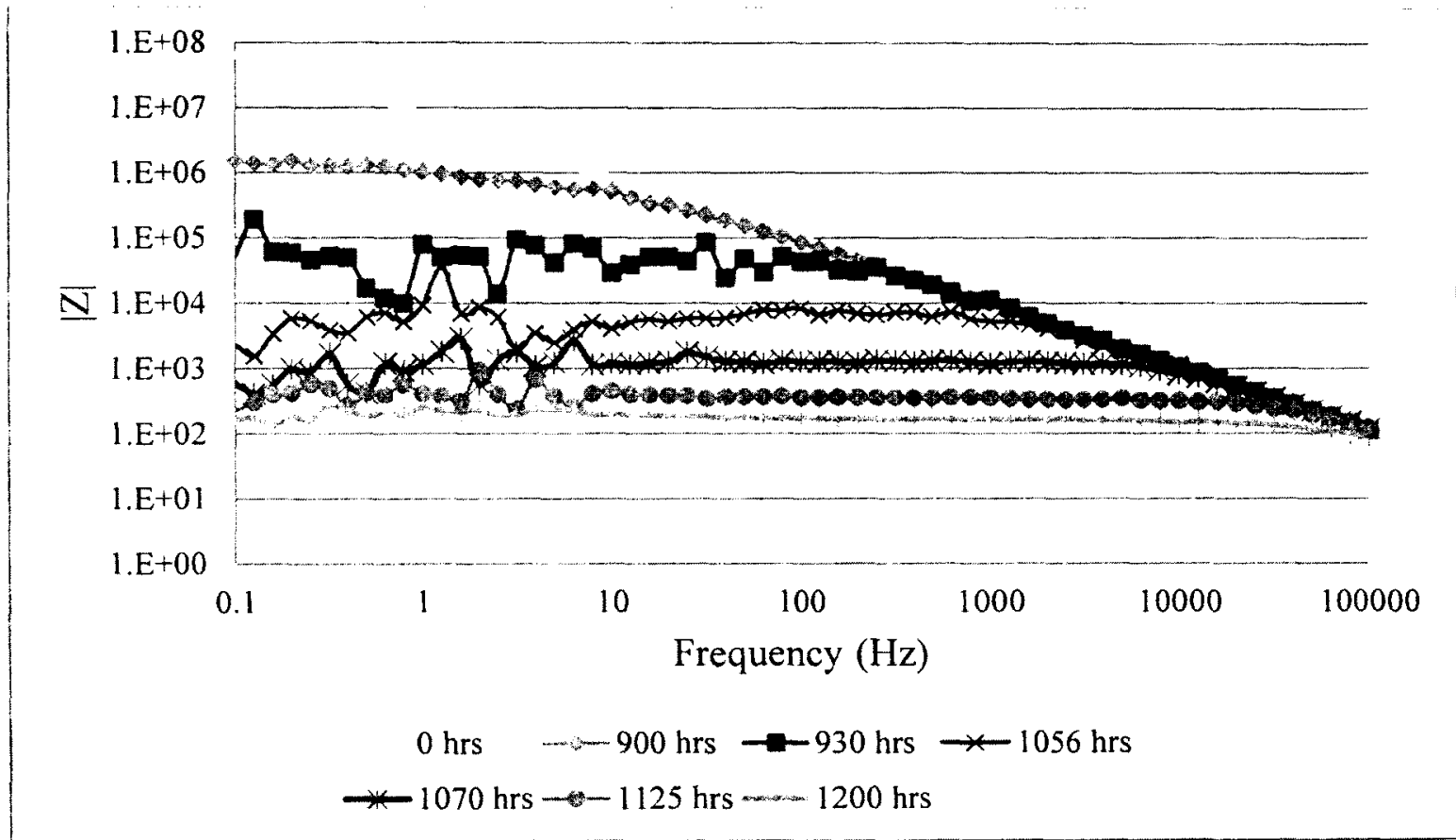

Figure 6-28 Impedance $(\Omega)$ measurements using Gamry $1\left(\mathrm{VC}, 34.0 \pm 0.5 \mathrm{~cm}^{2}\right)$ for a coated aluminum sample in $1 \mathrm{MHCl}(\mathrm{pH} \mathrm{0.1})$. 


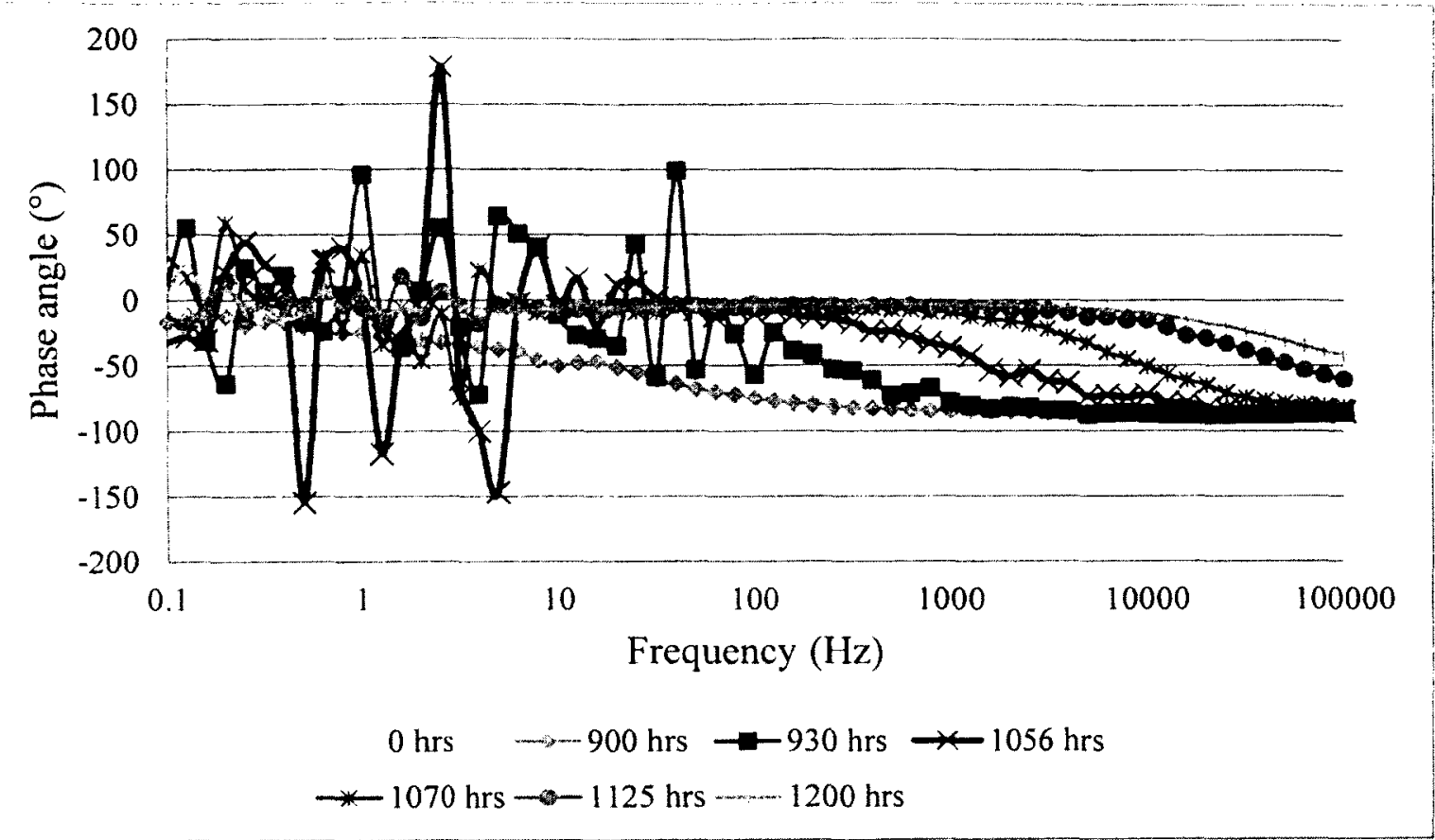

Figure 6-29 Phase angle measurements using Gamry $1\left(\mathrm{VC}, 34.0 \pm 0.5 \mathrm{~cm}^{2}\right)$ for coated aluminum sample in $1 \mathrm{M} \mathrm{HCl}(\mathrm{pH} 0.1)$.

Similar to the samples exposed to $\mathrm{NaCl}$ solution, bubbles were observed forming on the surface of the sample. The number of bubbles and pitting sites, however was much higher compared to the samples exposed to $\mathrm{NaCl}$ (Figure 6-30 and Figure 6-31). 


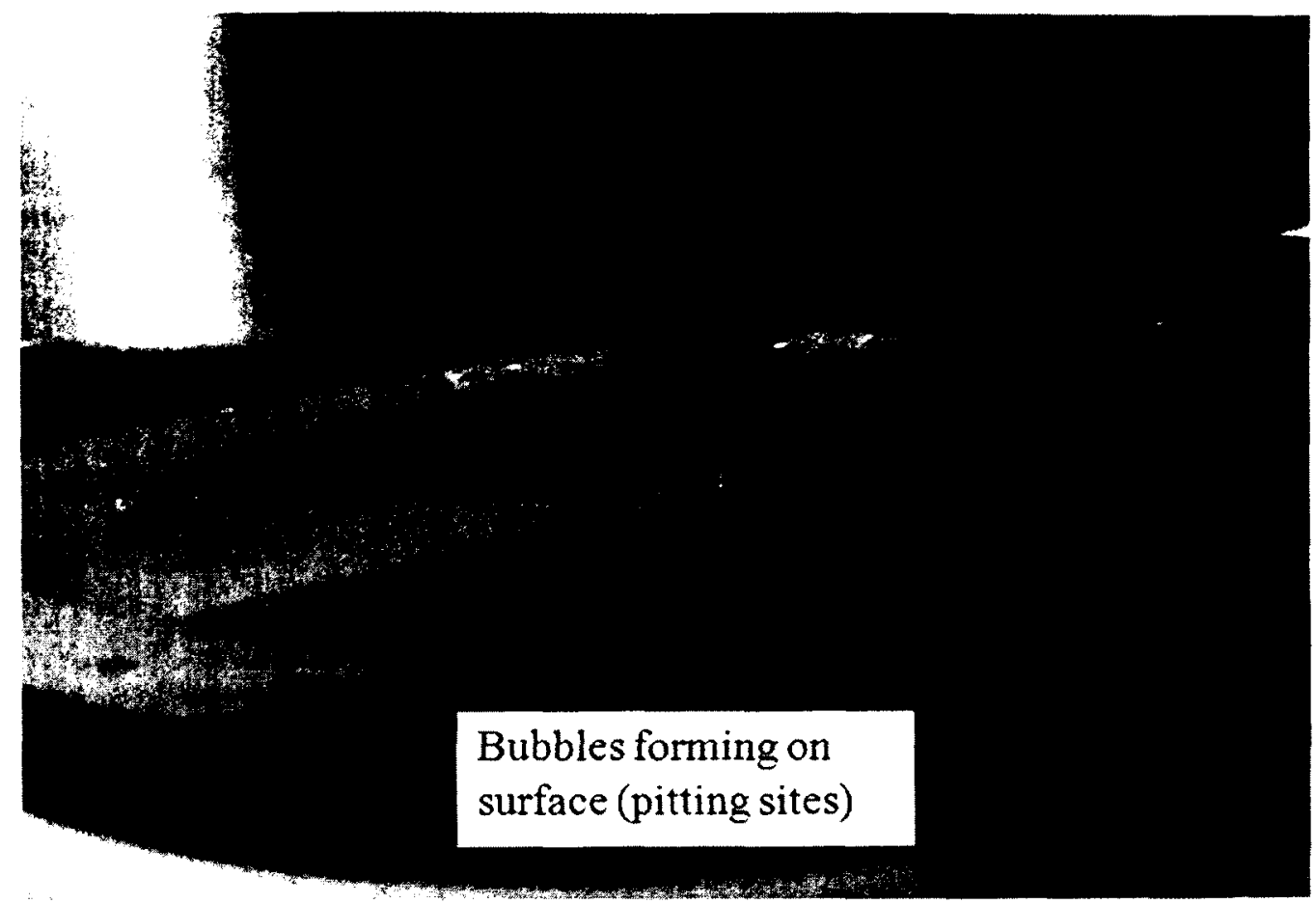

Figure 6-30 Bubbles starting to form on the surface of a sample exposed to $1 \mathrm{M} \mathrm{HCl}$ (pH 0.1) in the vertical cell (Figure 4-1).

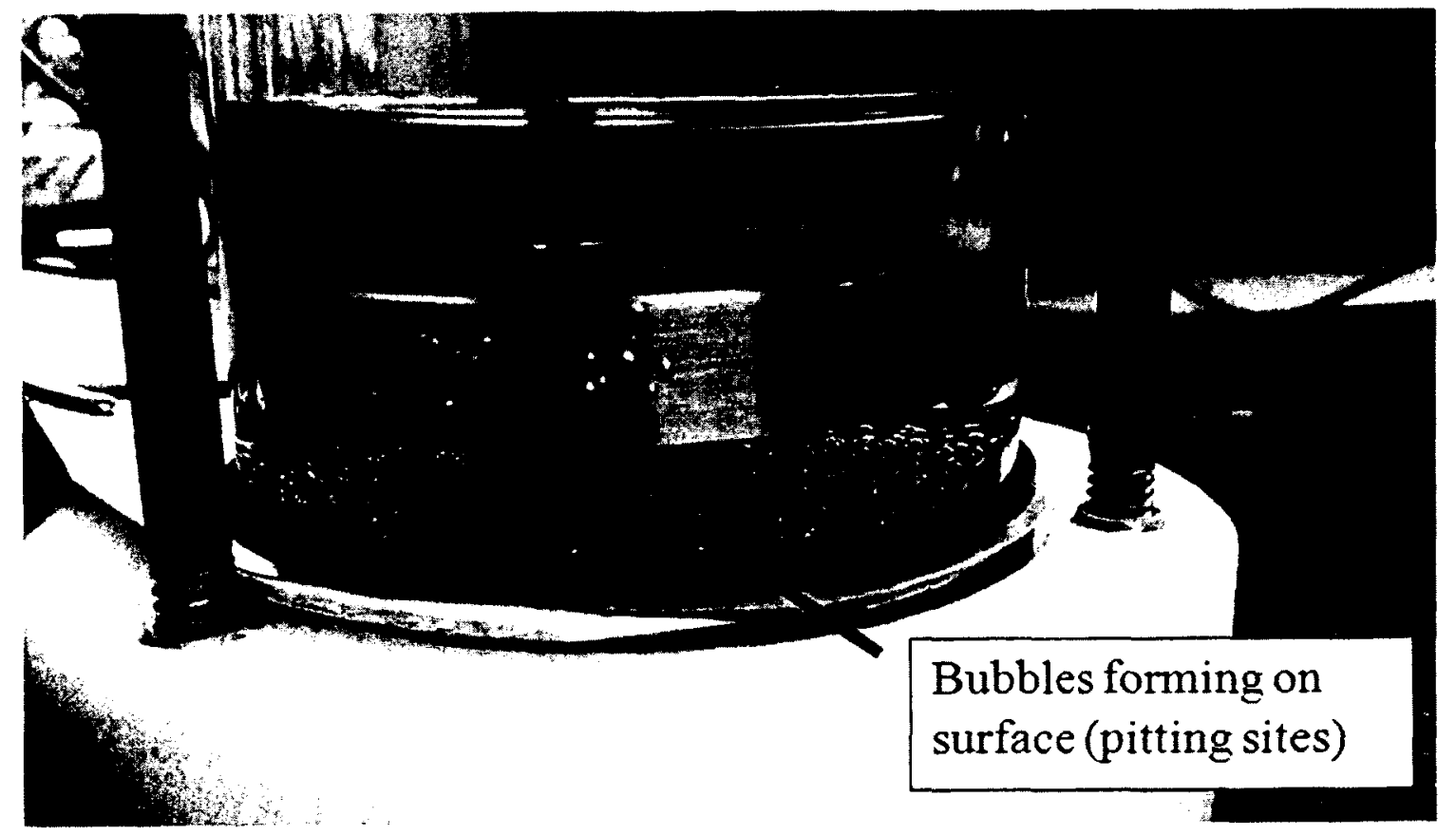

Figure 6-31 Bubbles on the surface of a sample exposed to $1 \mathrm{M} \mathrm{HCl}(\mathrm{pH} \mathrm{0.1)}$ in the vertical cell (Figure 4-1). 
After examining the sample, several pits and through holes were found on the surface, Figure 6-32. A SEM image was produced for a pitted site in the sample, Figure 6-33. The image shows that the original coating, which initially covered the pitted region, no longer exists; unlike the samples in $\mathrm{NaCl}$ (see Figure 6-25). It appears that in $\mathrm{pH} 0.1$ acid the coatings may have disappeared. This needs further investigation.

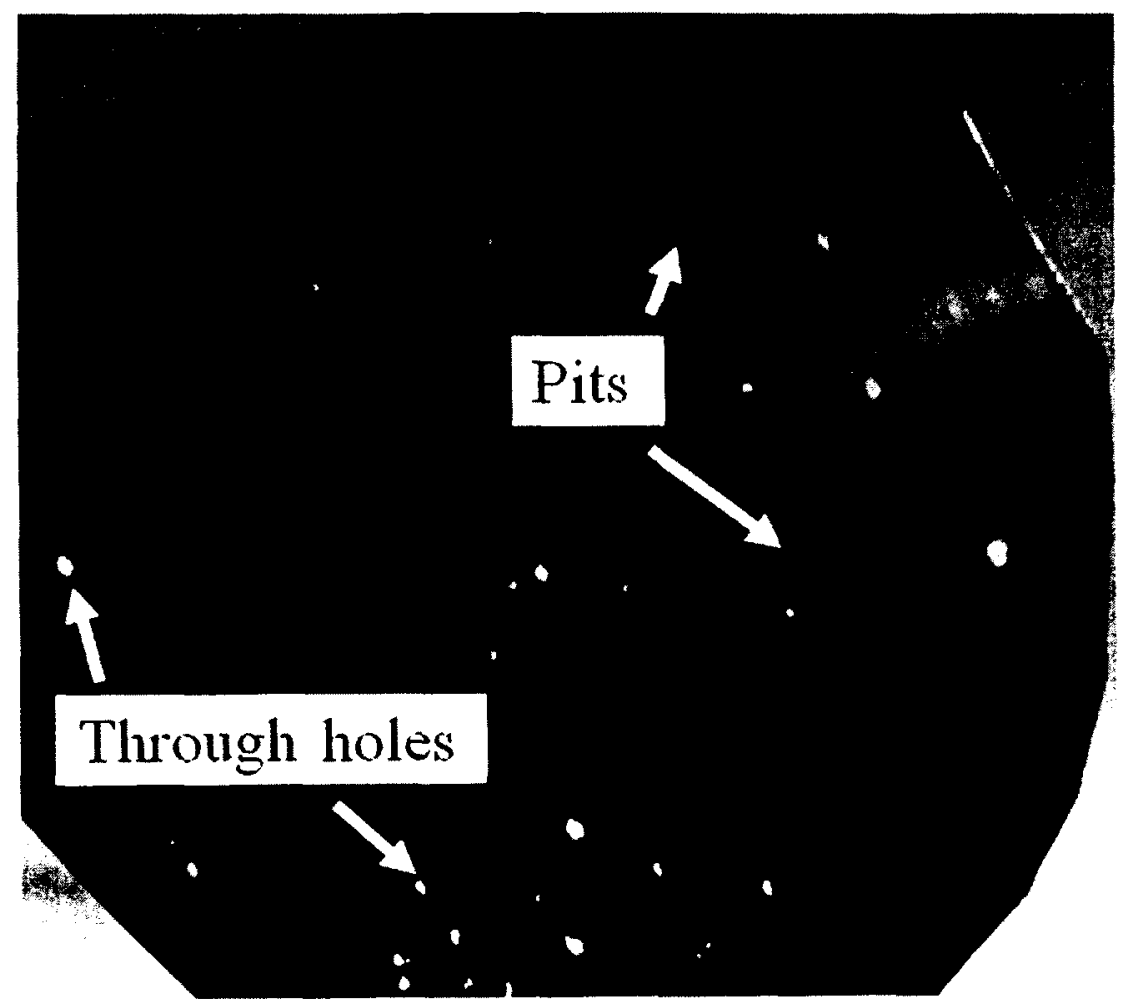

Figure 6-32 Condition of a coated aluminum sample after exposure to $1 \mathrm{M} \mathrm{HCl}$ (pH 0.1) for 1200 hours in the vertical cell (Figure 4-1). Many pits and through holes formed on the surface. 


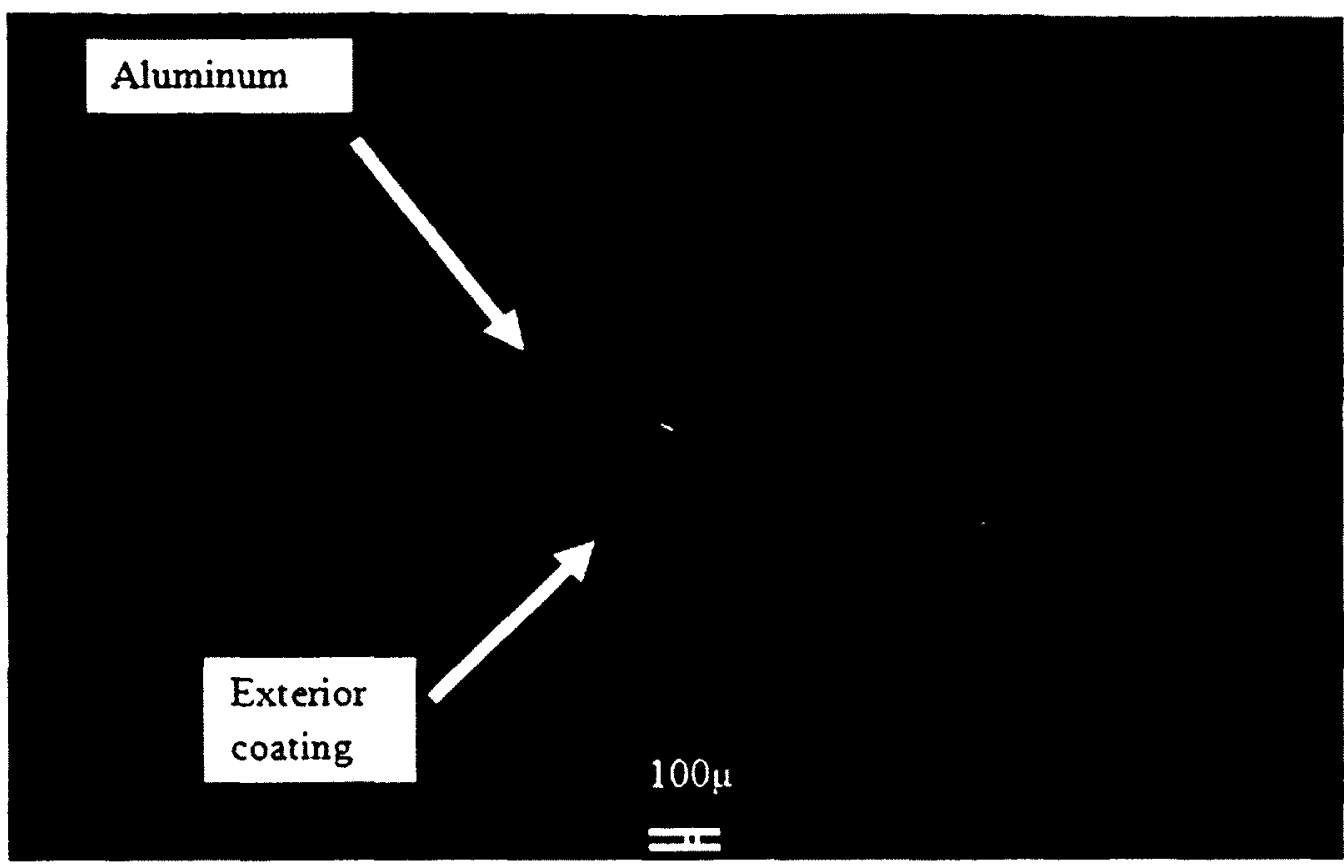

Figure 6-33 SEM image of a pit in the sample exposed to $1 \mathrm{M} \mathrm{HCl}(\mathrm{pH} 0.1)$. Note the state of the coating around the pitting site: Some of the coating appears to be missing.

\subsubsection{2. $\mathrm{pH} 2.6 \mathrm{HCl}$}

Once it was known how the samples performed in low $\mathrm{pH}$ environments and neutral environments, the samples were then tested at a $\mathrm{pH}$ near service conditions. $\mathrm{HCl}$ acid solution was prepared and the $\mathrm{pH}$ was adjusted to a value of 2.6 , which is similar to canned beverages (as measured in the laboratory). A sample was placed in the horizontal cell, and EIS data were collected using Solartron 2.

Figure 6-34 and Figure 6-35 show some of the EIS data for this sample. Upon initial immersion, this sample had an impedance of $>10^{8} \Omega$ at $1 \mathrm{~Hz}$ and a phase angle of $-90^{\circ}$ across the frequency range, indicating an intact coating. Once again, due to the potentiostat being pushed to its measuring limits, spikes were observed in the impedance and phase plots. 
After 53 hours of immersion, a drastic drop was observed in the impedance plot, $10^{9} \Omega$ down to $10^{6} \Omega$ at $0.01 \mathrm{~Hz}$. This early drop in impedance was indicative that the coating had a weak spot that was penetrated by the electrolyte.

The phase plot showed a mostly resistive behavior which indicated that a breach of the coating had taken place and the metal substrate should be under chemical attack.

Samples exposed to neutral solutions $(\mathrm{pH} \sim 8)$ intermittently passivated causing the impedance to rise at times, while samples in aggressive acids $(\mathrm{pH} \sim 0.1)$ did not passivate at all. The current sample was in a solution of $\mathrm{pH} \sim 2.6$. Upon examining the Pourbaix diagram, Figure 3-2, it is not obvious whether this sample should passivate or not, i.e., whether aluminum oxide is sufficiently stable. By looking at the impedance plot, Figure $6-34$, at 70 hours, it is noted that the impedance starts to rise at the low end of the frequency spectrum, $0.1 \mathrm{~Hz}$. At 86 hours, the impedance measurements are comparable to the impedance measurements at 0 hours, indicating the occurrence of passivation.

By looking at the data up to 255 hours of immersion (Figure 6-34-Figure 6-39), this sample's performance is seen to resemble that of a sample in neutral solution; passivation occurring intermittently with a general drop in the impedance and an increasing capacitive-to-resistive transition frequency. 


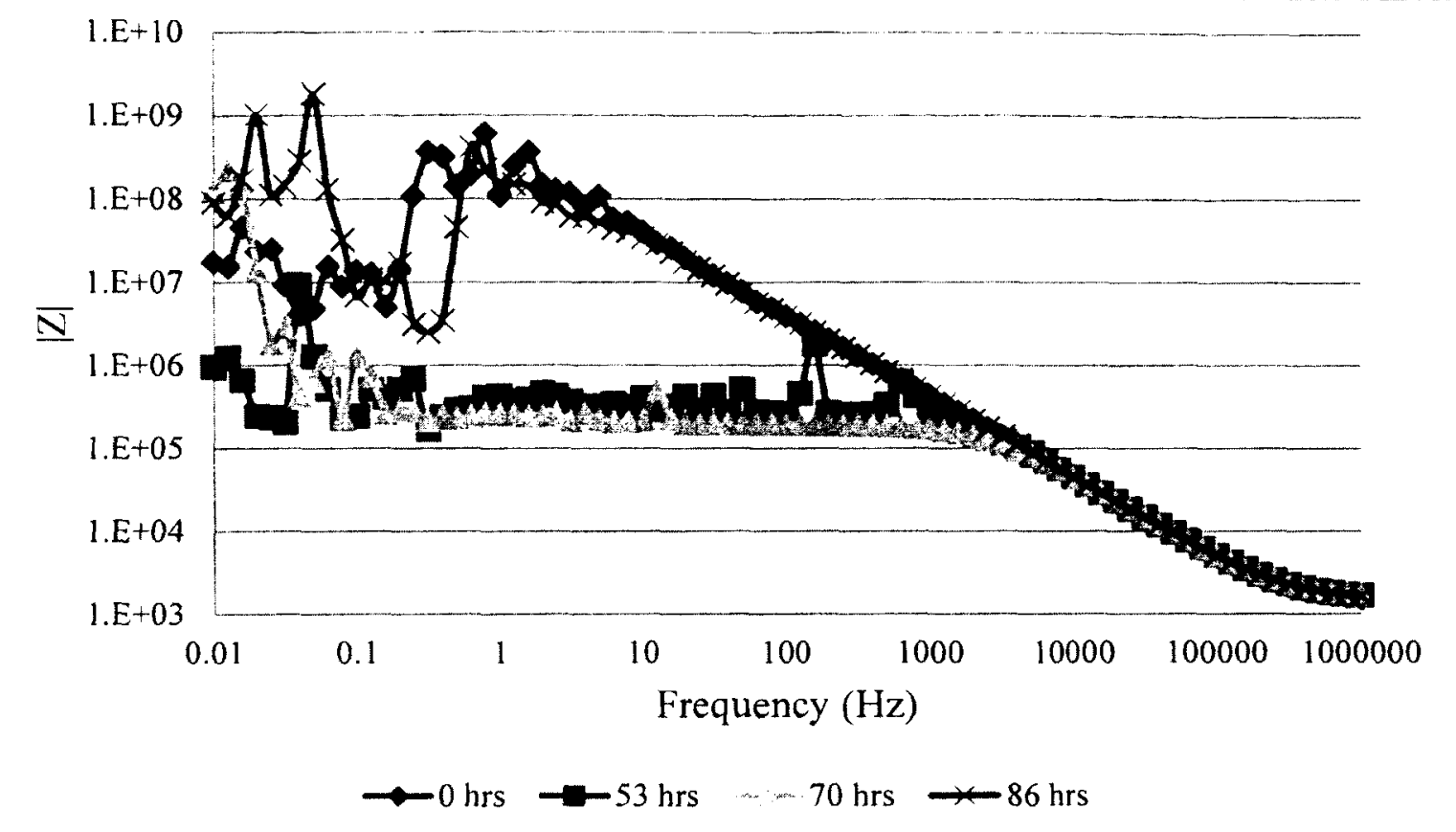

Figure 6-34 Impedance $(\Omega)$ measurements using Solartron $2\left(\mathrm{HC}, 1.0 \pm 0.1 \mathrm{~cm}^{2}\right)$ for coated aluminum sample in $\mathrm{HCl}$ (pH 2.6). Note the drop in impedance at 53 hours of exposure, probably indicating a pre-existing flaw.

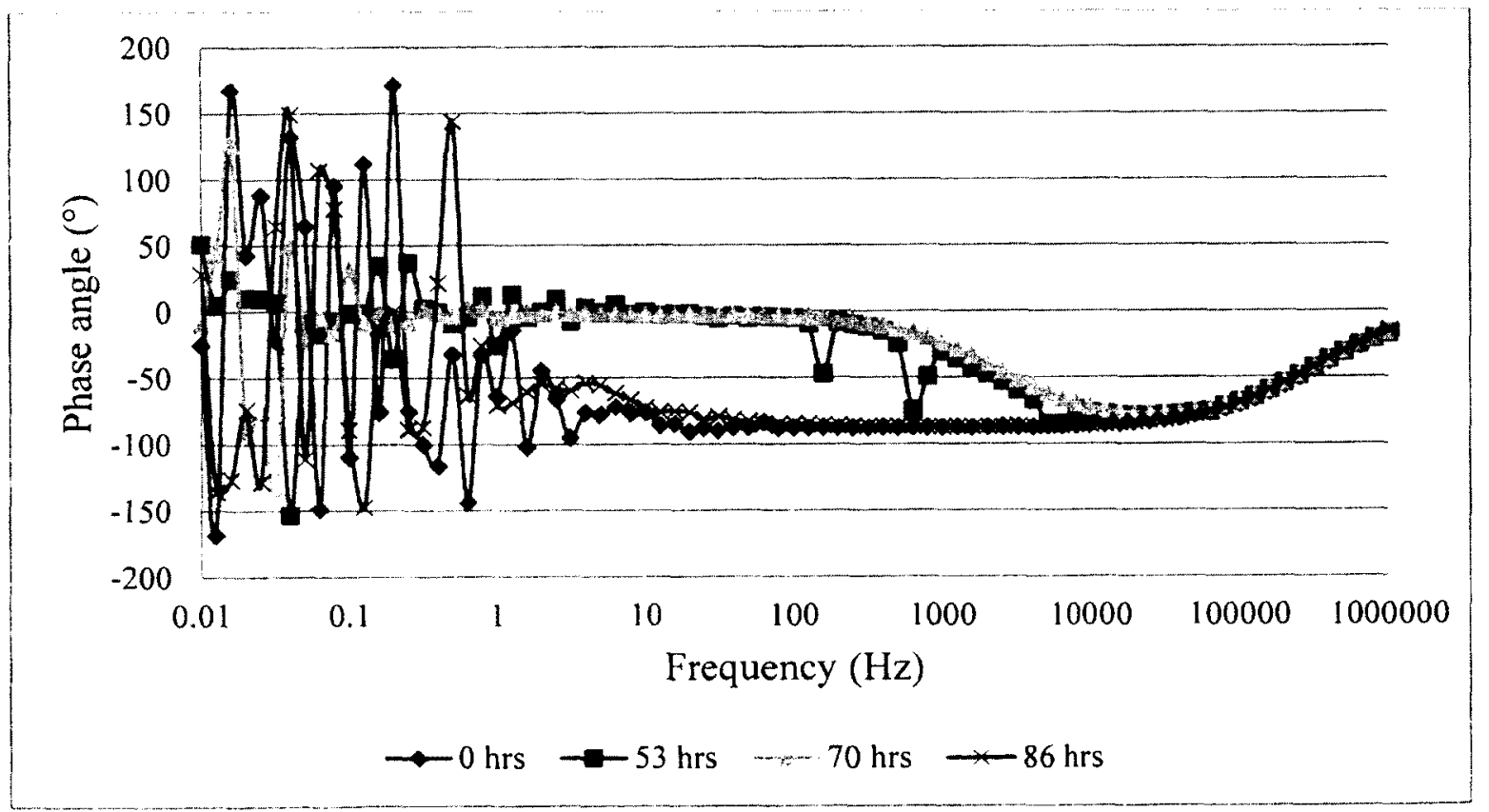

Figure 6-35 Phase angle measurements using Solartron $2\left(\mathrm{HC}, 1.0 \pm 0.1 \mathrm{~cm}^{2}\right)$ for coated aluminum sample in $\mathrm{HCl}$ ( $\mathrm{pH} \mathrm{2.6)}$. 


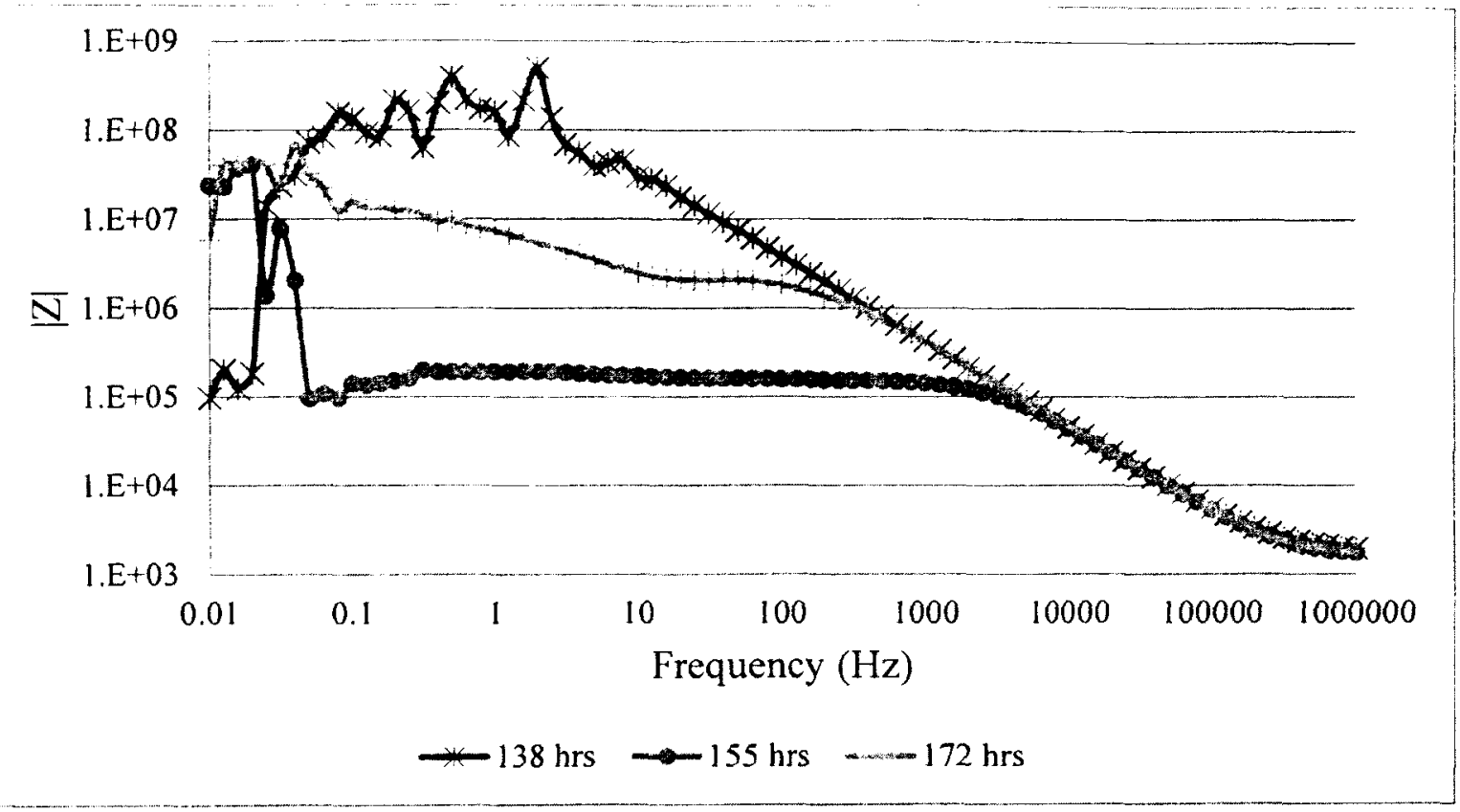

Figure 6-36 Impedance $(\Omega)$ measurements using Solartron $2\left(\mathrm{HC}, 1.0 \pm 0.1 \mathrm{~cm}^{2}\right)$ for coated aluminum sample in $\mathrm{HCl}$ (pH 2.6). Note the drop in impedance (breach in the oxide) at $138 \mathrm{hrs}$ and the increase in impedance (passivation) at 155 hrs.

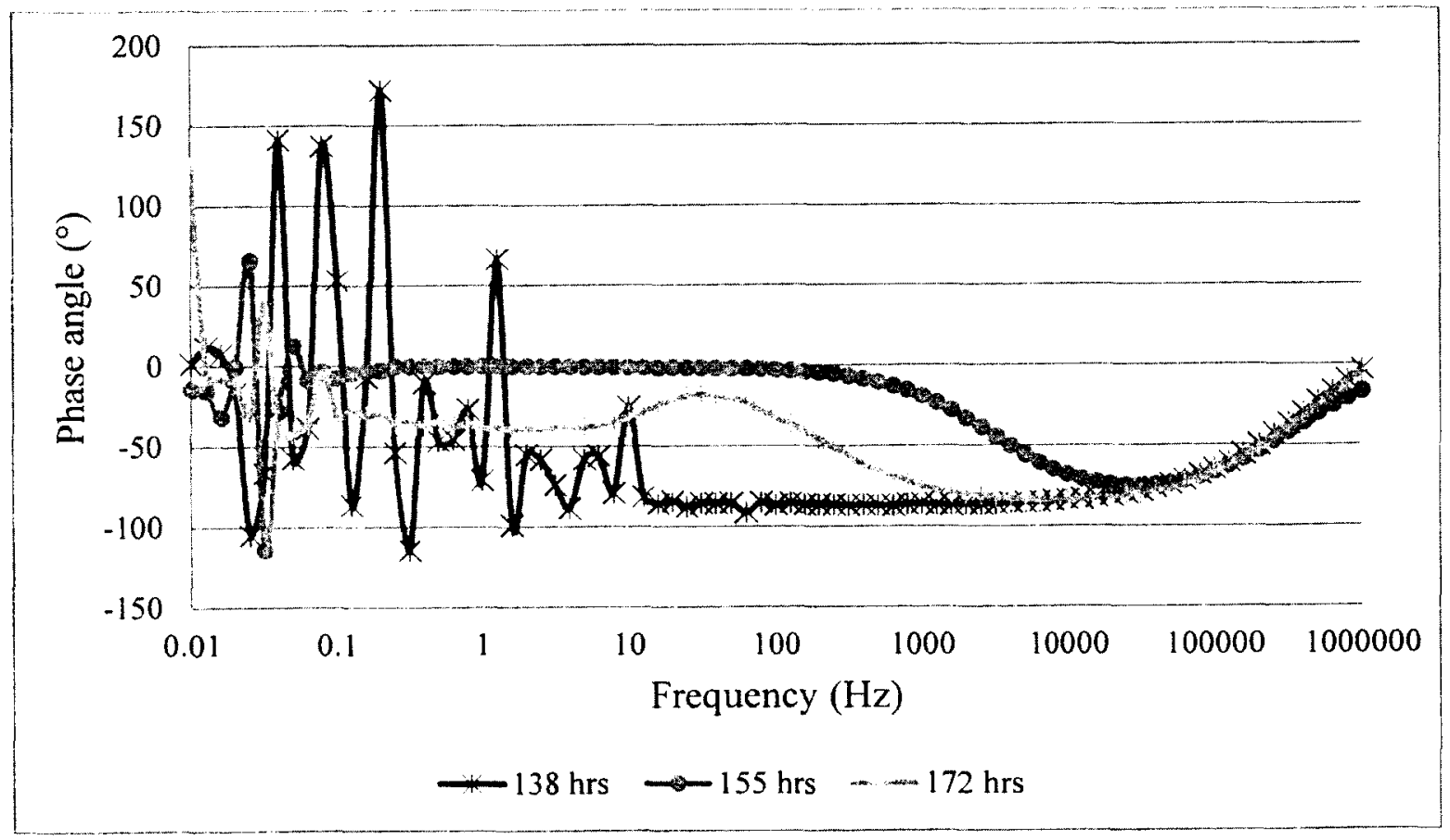

Figure 6-37 Phase angle measurements using Solartron $2\left(\mathrm{HC}, 1.0 \pm 0.1 \mathrm{~cm}^{2}\right)$ for coated aluminum sample in $\mathrm{HCl}$ (pH 2.6). 
After 288 hours of immersion, an increase in the high frequency $(>1000 \mathrm{~Hz})$ impedance was observed. This increase in impedance continued for approximately 150 hours, after which it returned to its original value at 437 hours, Figure 6-38. At 497 hours, the high frequency impedance increased again and remained for the duration of the experiment; until 650 hours no changes were observed. Such an increase in impedance was also observed when aluminum-foil was exposed to $1 \mathrm{M} \mathrm{NaCl}$, Figure 5-23.

By looking at Figure 6-38 and Figure 6-39, at 288 hours, not only does the high frequency impedance change, but the impedance and phase plots are increasing in complexity. However, this was not always observed. Due to the complexity of the impedance and phase plots, and the different kinds of corrosion that may be occurring, several explanations may be hypothesized for such occurrences. For the present engineering purposes, it is sufficient to know that an increase in the complexity of the EIS data, corrosion of some kind is occurring: the coating has failed. 


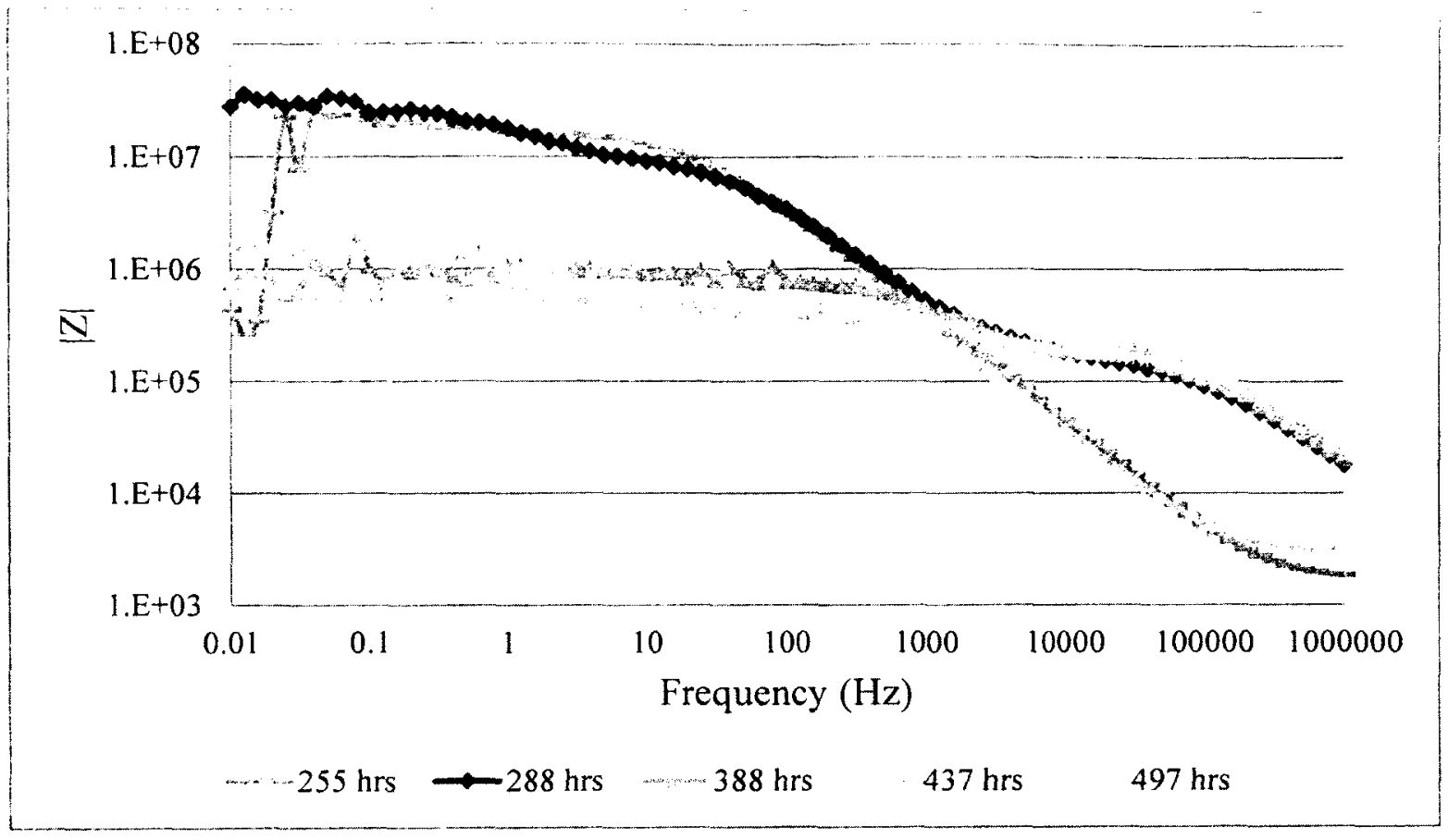

Figure 6-38 Impedance $(\Omega)$ measurements using Solartron $2\left(\mathrm{HC}, 1.0 \pm 0.1 \mathrm{~cm}^{2}\right)$ for coated aluminum sample in $\mathrm{HCl}(\mathrm{pH} \mathrm{2.6)}$. Note the increase in highfrequency impedance at $288 \mathrm{hrs}$ and the increasing complexity of the plot. 


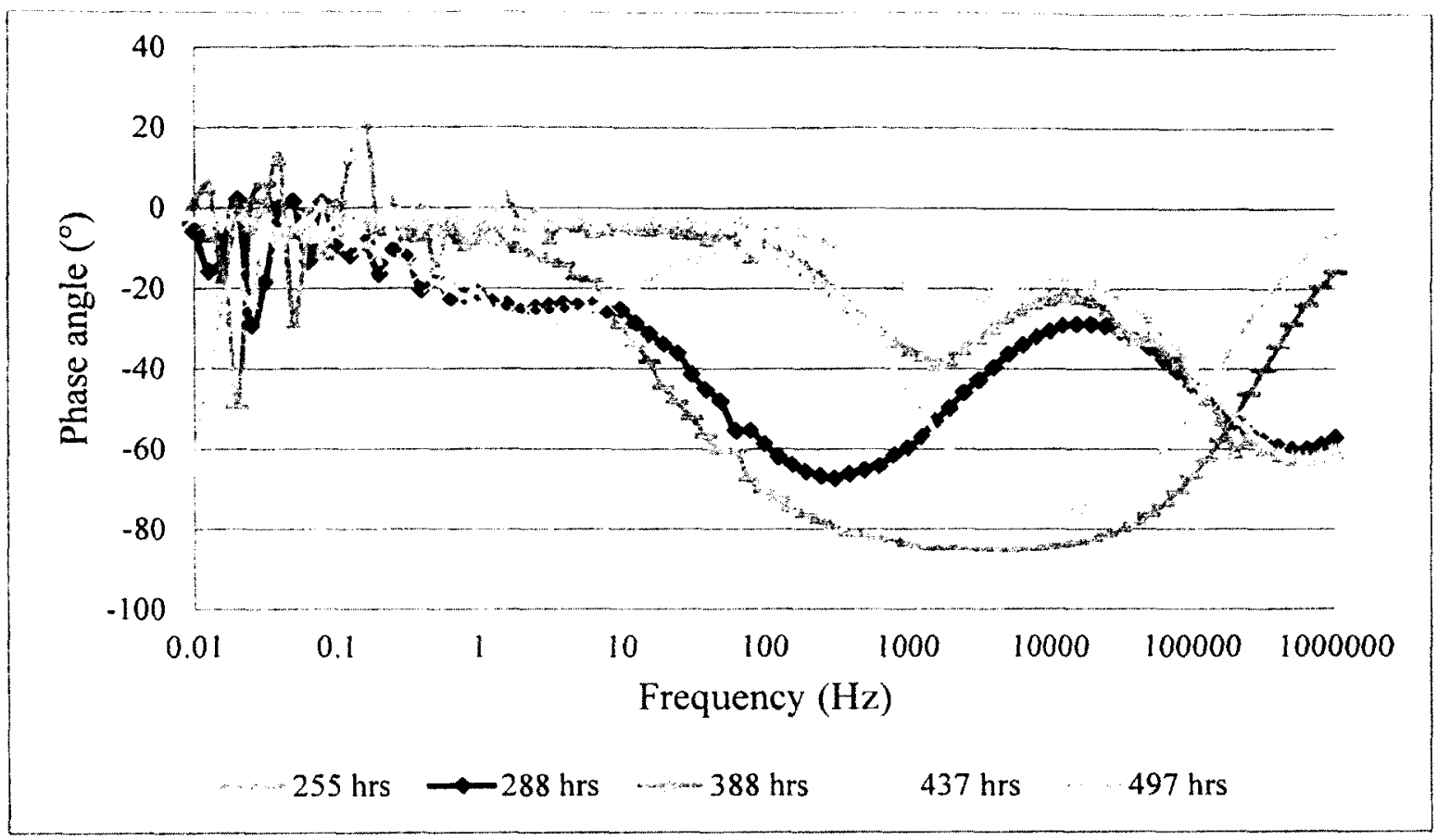

Figure 6-39 Phase angle measurements using Solartron $2\left(\mathrm{HC}, 1.0 \pm 0.1 \mathrm{~cm}^{2}\right)$ for coated aluminum sample in $\mathrm{HCl}$ ( $\mathrm{pH}$ 2.6). Note the increasing complexity in the phase plot.

The experiment was stopped after 650 hours of exposure to examine the sample. Several pits were found to have formed on the surface, and a white substance was observed in these pits; Figure 6-40. It is speculated that this white material is the 'stable' oxide forming in the pits. During this experiment, no oxide cloud was found in the solution and the sample was not penetrated. 


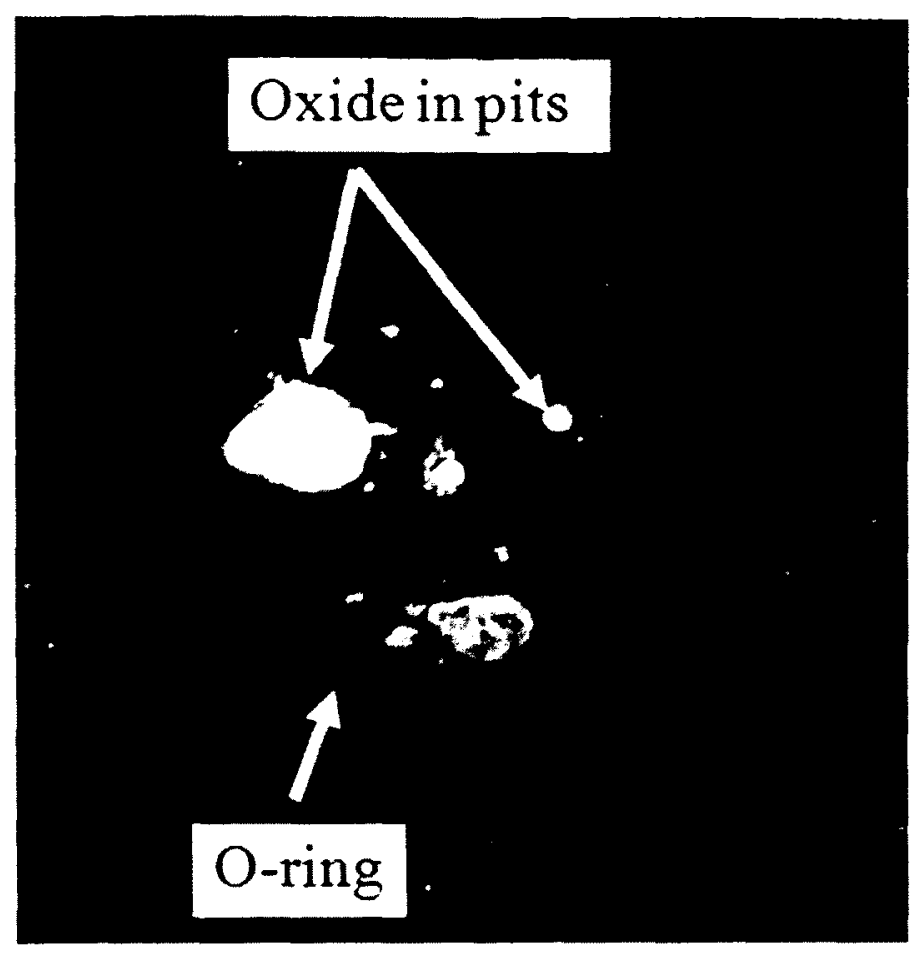

Figure 6-40 Condition of a coated aluminum sample after exposure to pH $2.6 \mathrm{HCl}$ for 650 hours in the horizontal cell (Figure 4-2). Note the oxide formed in the pits. There was no penetration.

\subsubsection{Polarization}

As demonstrated in section 5.3.5, preliminary experiments, the application of a DC voltage can turn corrosion on or off in the aluminum foil experiments. Similar experiments were performed with the coated aluminum samples.

EIS measurements were made with an applied DC potential with a corroding sample in $\mathrm{pH} 0.2 \mathrm{HCl}$, Figure 6-41 and Figure 6-42. Before the application of the DC voltage, the sample had an impedance of $10^{4} \Omega$ at $0.1 \mathrm{~Hz}$, and a resistive phase angle of $\left(\sim 0^{\circ}\right)$ for a wide frequency interval. In the phase plot, spikes were evident at frequencies $<10 \mathrm{~Hz}$, which is indicative of a corroding sample.

Initially, a small DC voltage of $-0.1 \mathrm{~V}$ was applied and EIS data were collected. Figure 6-41 shows the impedance increased over the entire frequency range up to 
$10^{6} \Omega$ at $1 \mathrm{~Hz}$. Also, once the frequency reached $1 \mathrm{~Hz}$, a sharp rise in the impedance to $10^{6} \Omega$ was observed. Looking at the phase plot, Figure $6-42$, the spikes at the low frequencies have disappeared along with the positive phase angles, and capacitive traits are observed for a larger portion of the plot: capacitive to resistive transition frequency decreased.

This experiment with an applied potential of $-0.1 \mathrm{~V}$ was followed by an application of -1 V DC. The application of such a voltage led to another increase in the impedance, $>10^{6} \Omega$ at $0.1 \mathrm{~Hz}$. Both the impedance and phase plots demonstrated capacitive traits for a larger portion of the plots (see Figure 2-15). The disappearance of the positive phase angles during cathodic polarization supports the hypothesis that positive phase angles and spiking are indicative of corrosion (see section 5.3).

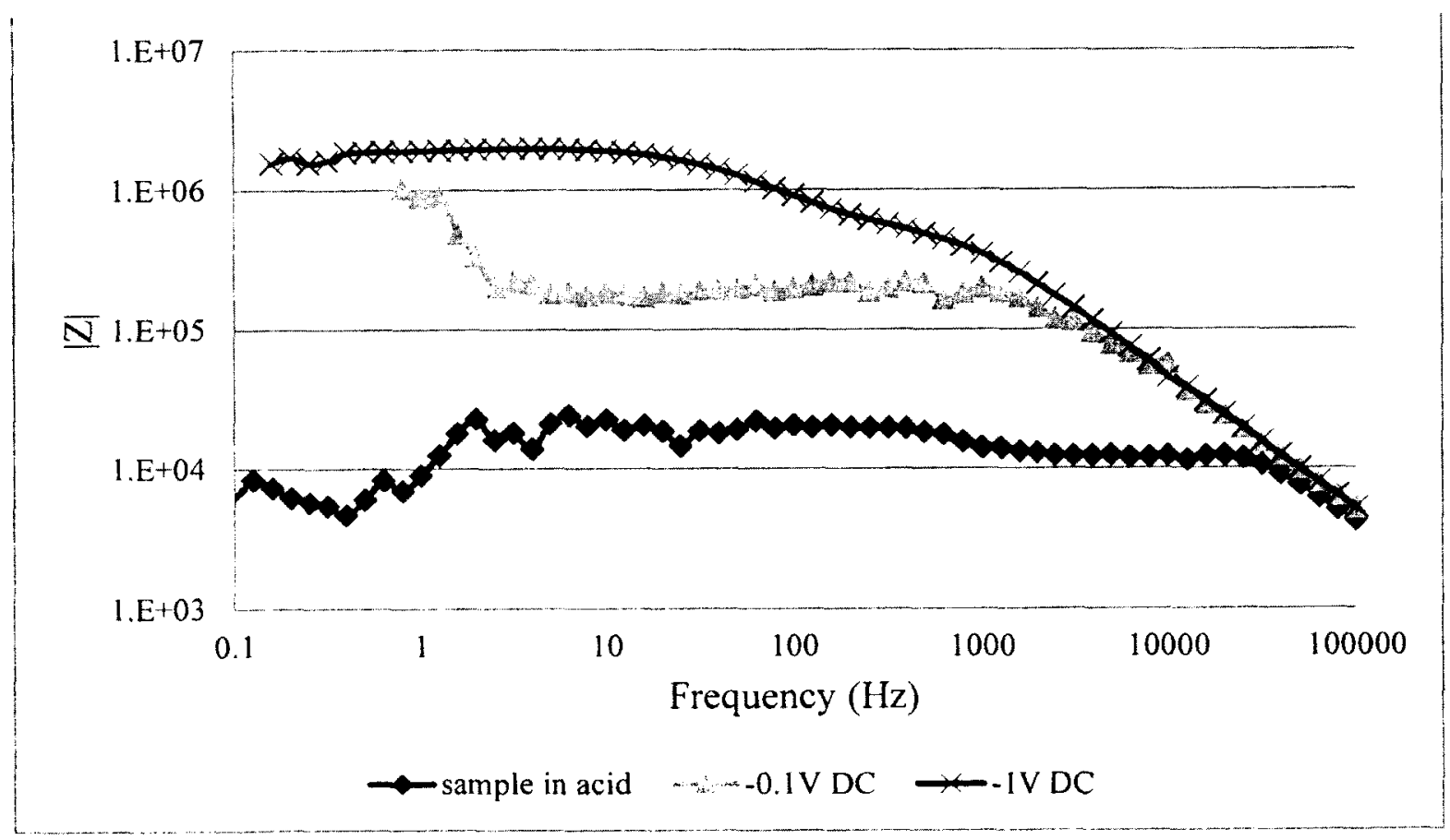

Figure 6-41 Impedance ( $\Omega$ ) measurements using Gamry $1\left(\mathrm{HC}, 1.0 \pm 0.1 \mathrm{~cm}^{2}\right)$ demonstrating the effects of applying small $D C$ voltages to coated aluminum in $\mathrm{pH} 0.2 \mathrm{HCl}$. Note the increase in impedance with the application of a cathodic (negative) voltage. 


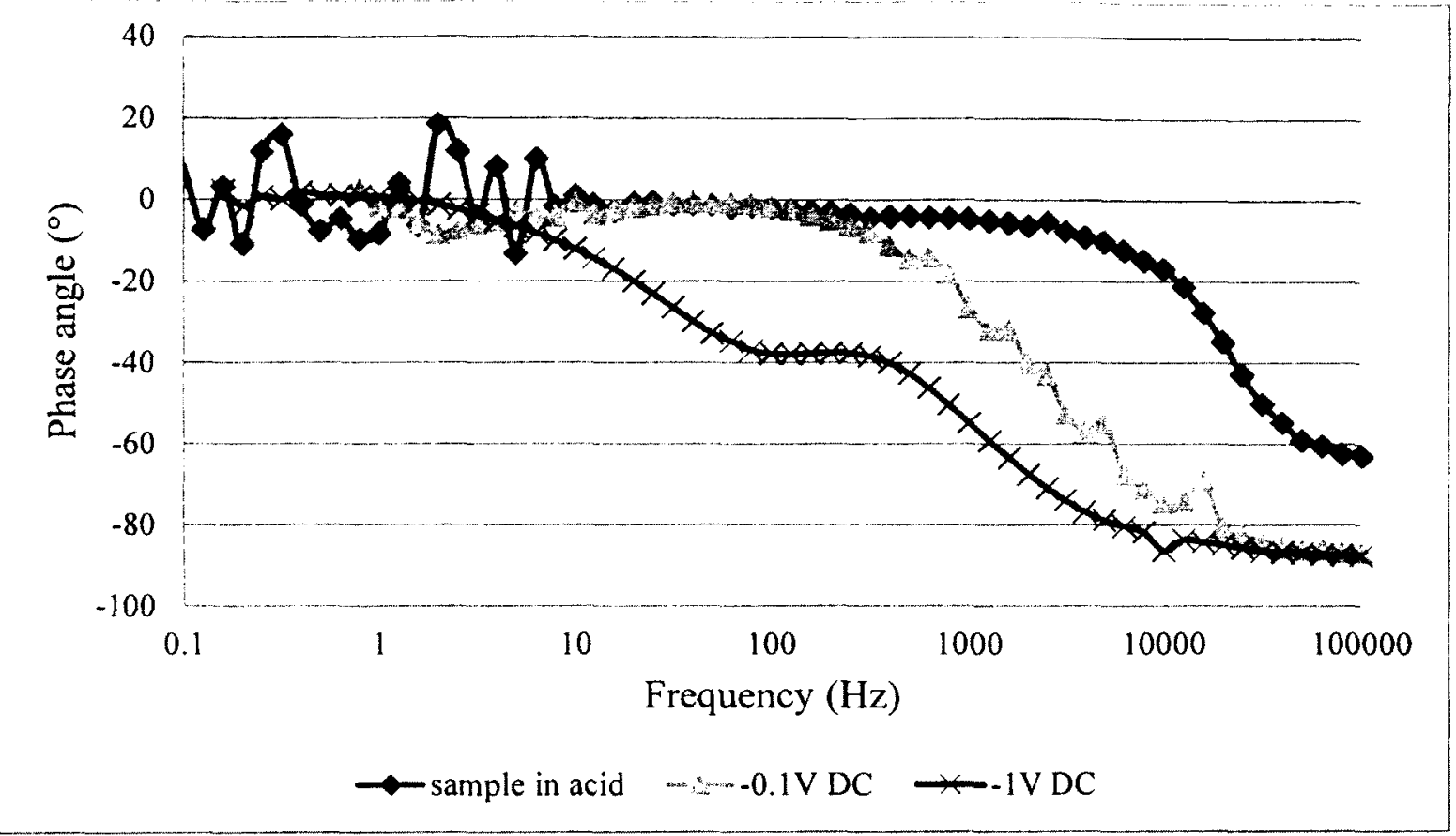

Figure 6-42 Phase angle measurements using Gamry 1 (HC, $1.0 \pm 0.1 \mathrm{~cm}^{2}$ ) demonstrating the effects of applying small DC voltages to coated aluminum in pH $0.2 \mathrm{HCl}$. With the application of a cathodic (negative) voltage, spikes disappeared and the phase plots are more capacitive.

\subsection{Experiments in Deaerated Solutions}

\subsubsection{Introduction}

In all the EIS experiments performed in aerated solutions, the coatings failed within a timeframe varying from instantaneous (flawed samples), two weeks (in neutral $\mathrm{pH}$ ) and two months (in acidic pH). In all cases, pitting was observed, often with complete penetration of the aluminum.

During service, coatings in beverage cans typically display a much longer shelf life before any failure is observed. These cans contain beverages that are saturated in dissolved $\mathrm{CO}_{2}$ and contain no oxygen. From the literature it is known that the oxygen content has a great effect on corrosion rate (see Section 3.3.2). In order to test the effect 
of dissolved $\mathrm{CO}_{2}$ and lack of oxygen, EIS experiments were conducted in deaerated solutions with and without $\mathrm{CO}_{2}$.

\subsection{2. $1 \mathrm{M} \mathrm{NaCl}$ (pH 8) Deaerated with Dry Ice}

The first experiment was conducted in deaerated $1 \mathrm{M} \mathrm{NaCl}(\mathrm{pH} \sim 8$ ) solution using Solartron 1. Dry ice was added until no further bubbling/reaction took place; the $\mathrm{CO}_{2}$ from the dry ice was assumed to have replaced all of the $\mathrm{O}_{2}$ in the solution. As soon as the solution was placed in the corrosion cell, the cell was sealed and EIS data were collected.

Figure 6-43 shows the impedance plot and Figure 6-44 shows the phase plot for the sample in deaerated $1 \mathrm{M} \mathrm{NaCl}$. This sample had a starting impedance of $10^{8} \Omega$ at $0.01 \mathrm{~Hz}$ and a phase plot of $-90^{\circ}$ for the frequency range down to $10 \mathrm{~Hz}$. Spikes are observed in both plots at frequencies below $10 \mathrm{~Hz}$, but as mentioned earlier in section 5.2.3, these spikes arise due to the potentiostat trying to produce measurements beyond its capabilities.

Samples exposed to $1 \mathrm{M} \mathrm{NaCl}$ normally fail within two weeks of exposure, however, no indicators of coating penetration were observed in either the impedance plot or the phase-angle plot. Usually, the impedance starts to drop at the low frequency end and an increase in the capacitive to resistive transition frequency is observed. In this case however, a small shift in the entire plot was observed in the impedance plot. It seemed that the impedance values were slightly decreasing across the frequency spectrum, but the impedance remained capacitive.

Such a decrease in the impedance may indicate an increase in the capacitance of the sample, given by equation 2-16. Several factors can contribute to the increase in $C$ : an 
increase in the dielectric constant $\varepsilon$ or a decrease in coating thickness $d$. Both factors are plausible. An increase in the dielectric constant can be the result of electrolyte diffusing into the coating. A decrease in the effective thickness can result from an attack on the coating. However, the most probable cause for this occurrence is electrolyte penetrating the coating, but due to the lack of oxygen, no corrosion has happened.

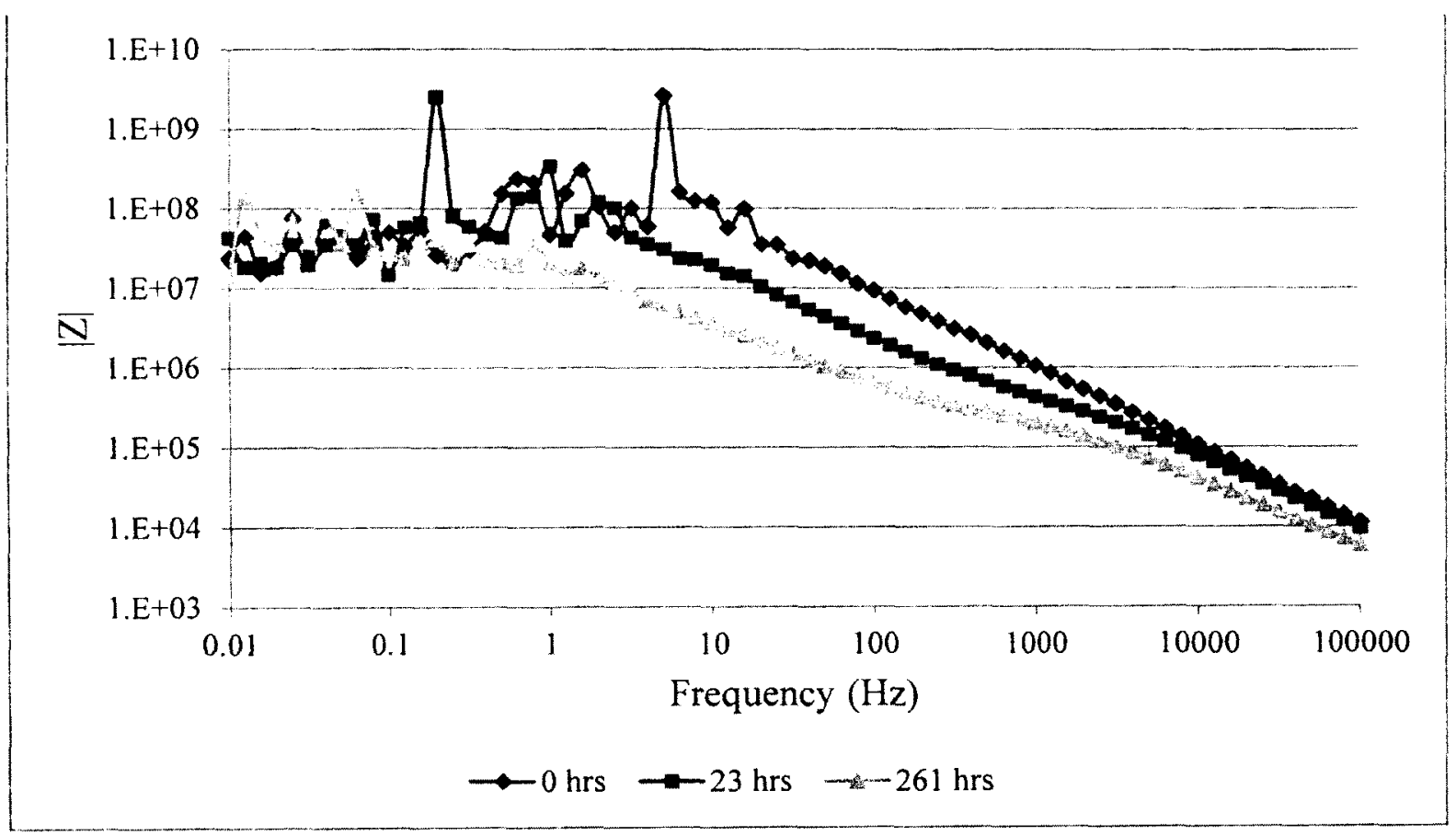

Figure 6-43 Impedance $(\Omega)$ measurements, using Solartron $1\left(\mathrm{HC}, 1.0 \pm 0.1 \mathrm{~cm}^{2}\right)$, for coated aluminum sample in deaerated $1 \mathrm{M} \mathrm{NaCl}(\mathrm{pH} 8)$. Note the downward shift in the impedance plot with exposure. 


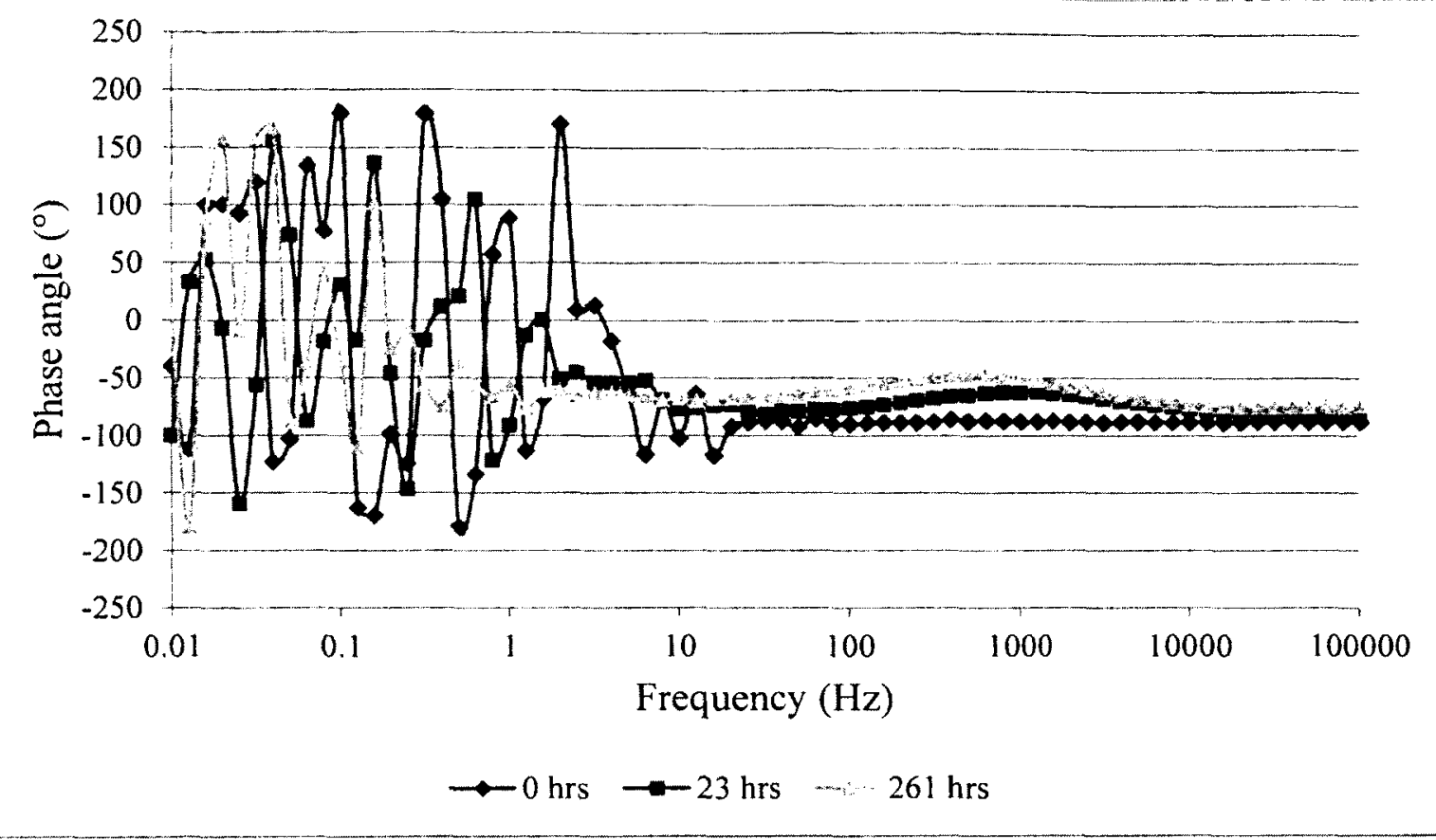

Figure 6-44 Phase angle measurements, using Solartron $1\left(\mathrm{HC}, 1.0 \pm 0.1 \mathrm{~cm}^{2}\right)$, for coated aluminum sample in deaerated $1 \mathrm{M} \mathrm{NaCl}$ (pH 8).

\subsection{3. $1 \mathrm{M} \mathrm{NaCl}(\mathrm{pH} \mathrm{8})$ Deaerated with Nitrogen Gas}

Similar to the previous section, a sample was tested in deaerated $1 \mathrm{M} \mathrm{NaCl}(\mathrm{pH} 8)$ solution. This time, the solution was deaerated with the use of $\mathrm{N}_{2}$ gas. The impedance and phase plots are shown in Figure 6-45 and Figure 6-46. This sample had a starting impedance of $10^{8} \Omega$ at $0.01 \mathrm{~Hz}$ and a phase angle of $-90^{\circ}$ for the entire frequency spectrum. Once again, spikes are observed in the plots due to the measuring limitations of the instrument at $10^{8} \Omega$.

For 1650 hours, no drops were observed in the impedance plot, except for a slight downward shift in the impedance; similar to the previous section. Two explanations were hypothesized for the shift in the impedance, a change in the effective dielectric constant or a change in the effective thickness of the coating. This kind of change with the 
impedance slowly dropping over the whole spectrum was not observed in any of the previous experiments in aerated neutral solutions.

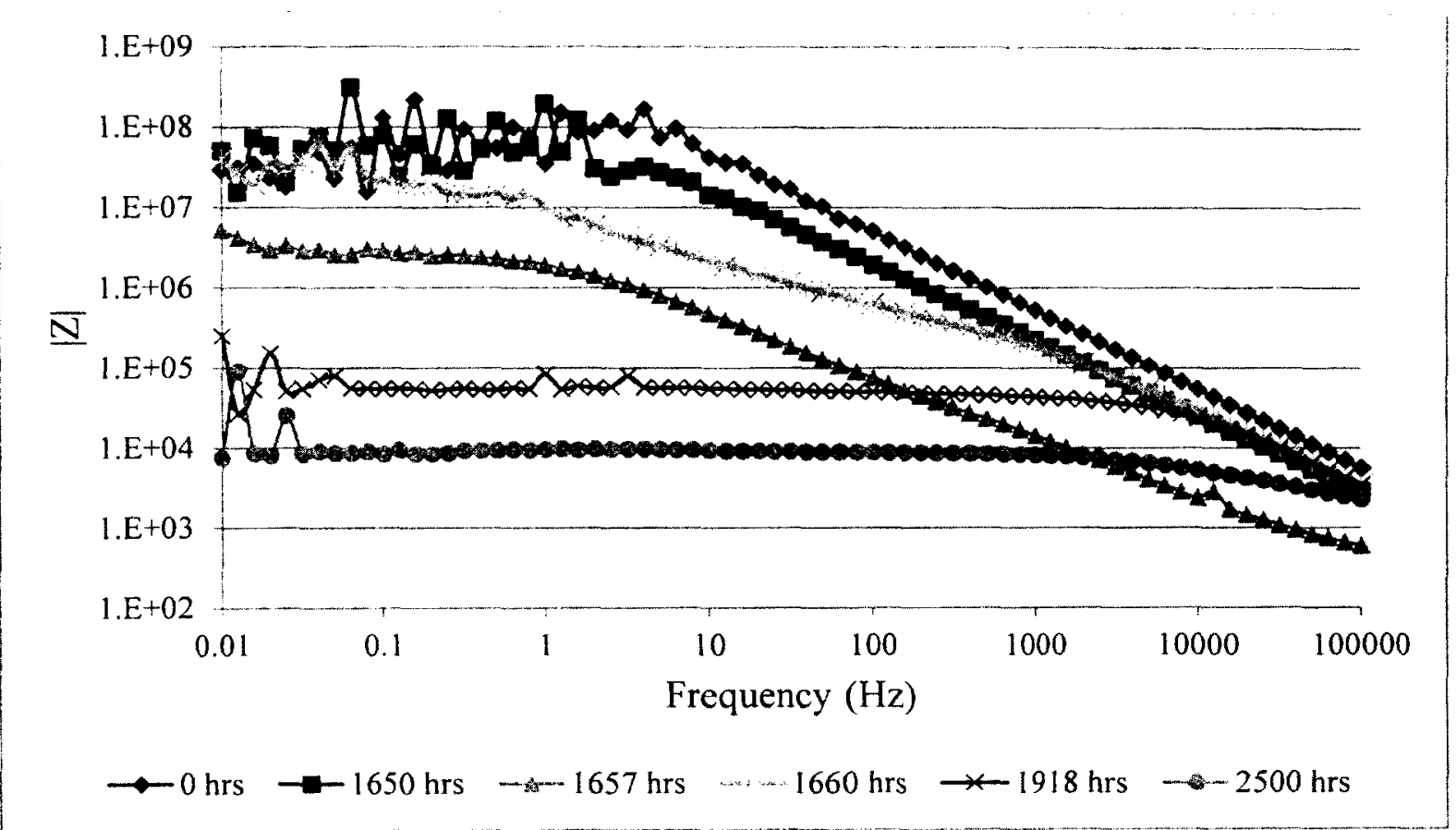

Figure 6-45 Impedance ( $\Omega$ ) measurements, using Solartron $1\left(\mathrm{HC}, 1.0 \pm 0.1 \mathrm{~cm}^{2}\right)$, for coated aluminum sample in deaerated $1 \mathrm{M} \mathrm{NaCl}(\mathrm{pH} \mathrm{8})$. Note the downward shift in impedance between 0 and 1650 hours of exposure. After aeration of the solution at 1657 hours, a large downward shift is observed in the impedance plot, 1657 hours, after which it shifted back.

After 1650 hours of exposure, the experiment was paused and the solution was aerated. EIS data at 1657 hours were collected as soon as the aeration process was complete. As shown in the impedance plot, after aeration, a significant downward shift was observed over the whole impedance curve. It appears that the capacitance of the coating suddenly increased and the coating resistance decreased. When the aeration process was started, the bubbled oxygen might have caused some passivating oxide to be blown away from the surface causing a drop in the impedance across the frequency spectrum. 
Now that the electrolyte was saturated with oxygen, in view of previous results in sections 6.2 and 6.3, one would anticipate corrosion to occur. As Figure 6-45 and Figure 6-46 demonstrate, soon after aeration, all the corrosion indicators could be observed: the low frequency impedance started to decrease; spiking occurred in the impedance and phase plots; the capacitive-to-resistive transition frequency increased; and positive phase angles appeared.

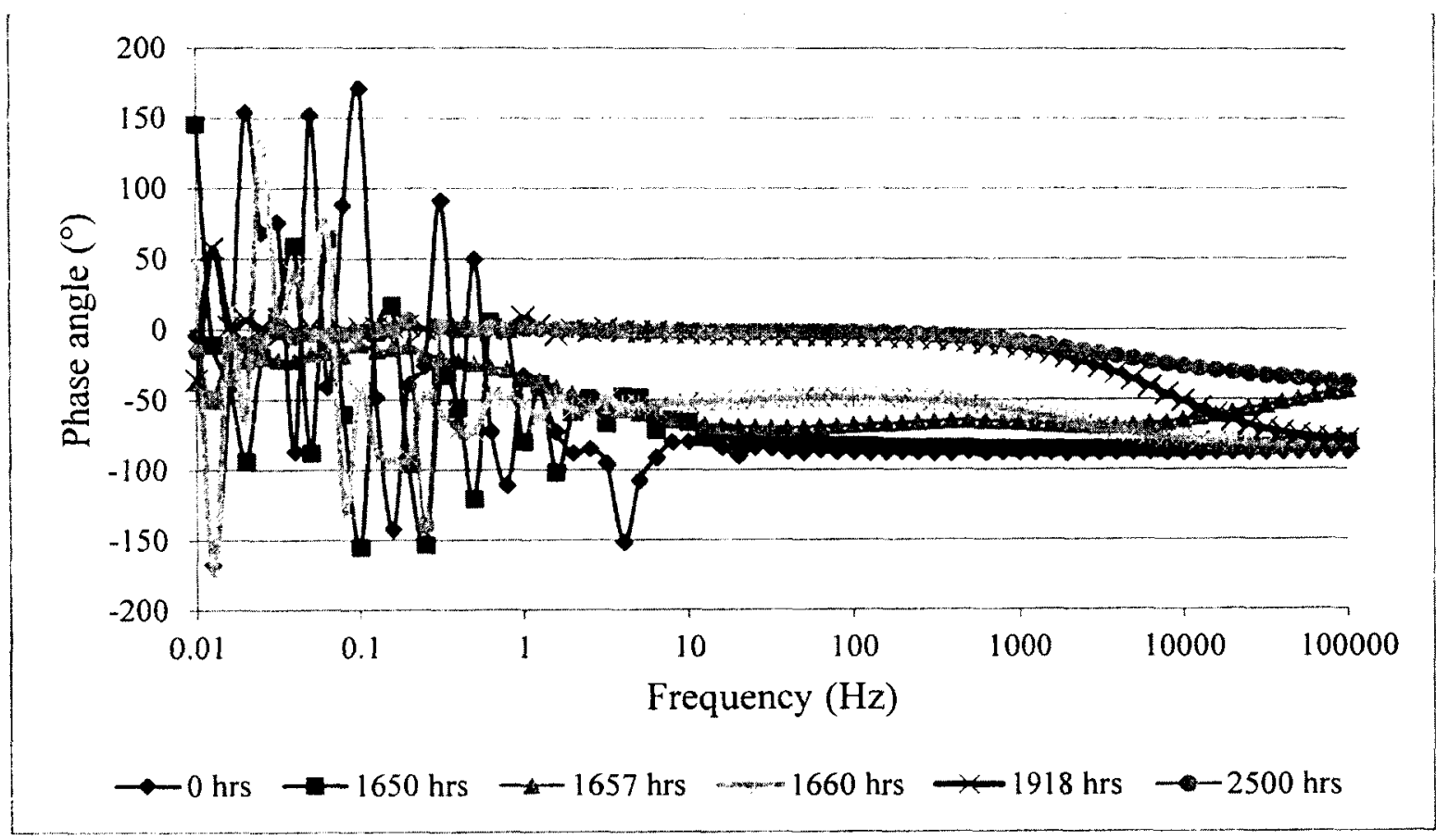

Figure 6-46 Phase angle measurements, using Solartron $1\left(\mathrm{HC}, 1.0 \pm 0.1 \mathrm{~cm}^{2}\right)$, for coated aluminum sample in deaerated $1 \mathrm{M} \mathrm{NaCl}(\mathrm{pH} \mathrm{8)}$. After aeration of the solution, a more resistive phase plot is observed, 1657 hours, which went back to being capacitive at 1660 hours.

During this experiment, gas bubbles and a white oxide cloud were observed only after aeration of the electrolyte. In addition, intermittent passivation was observed in the EIS data. After 2500 hours of exposure, the impedance indicated sample failure.

Upon inspection of the sample, it was found that the aluminum between the exterior (public side) and interior (beverage side) coatings had disappeared completely, 
Figure 6-47. Since the sample is bound by an O-ring, producing a compressive boundary, the solution was stopped at this boundary from travelling further across the sample and causing more corrosion.

The complete disappearance of the aluminum between the layers of coating is strong evidence for an acidic solution being produced in the actively corroding area where access to the bulk neutral pH solution is limited. This is in agreement with articles in the literature $[14-16,68]$.

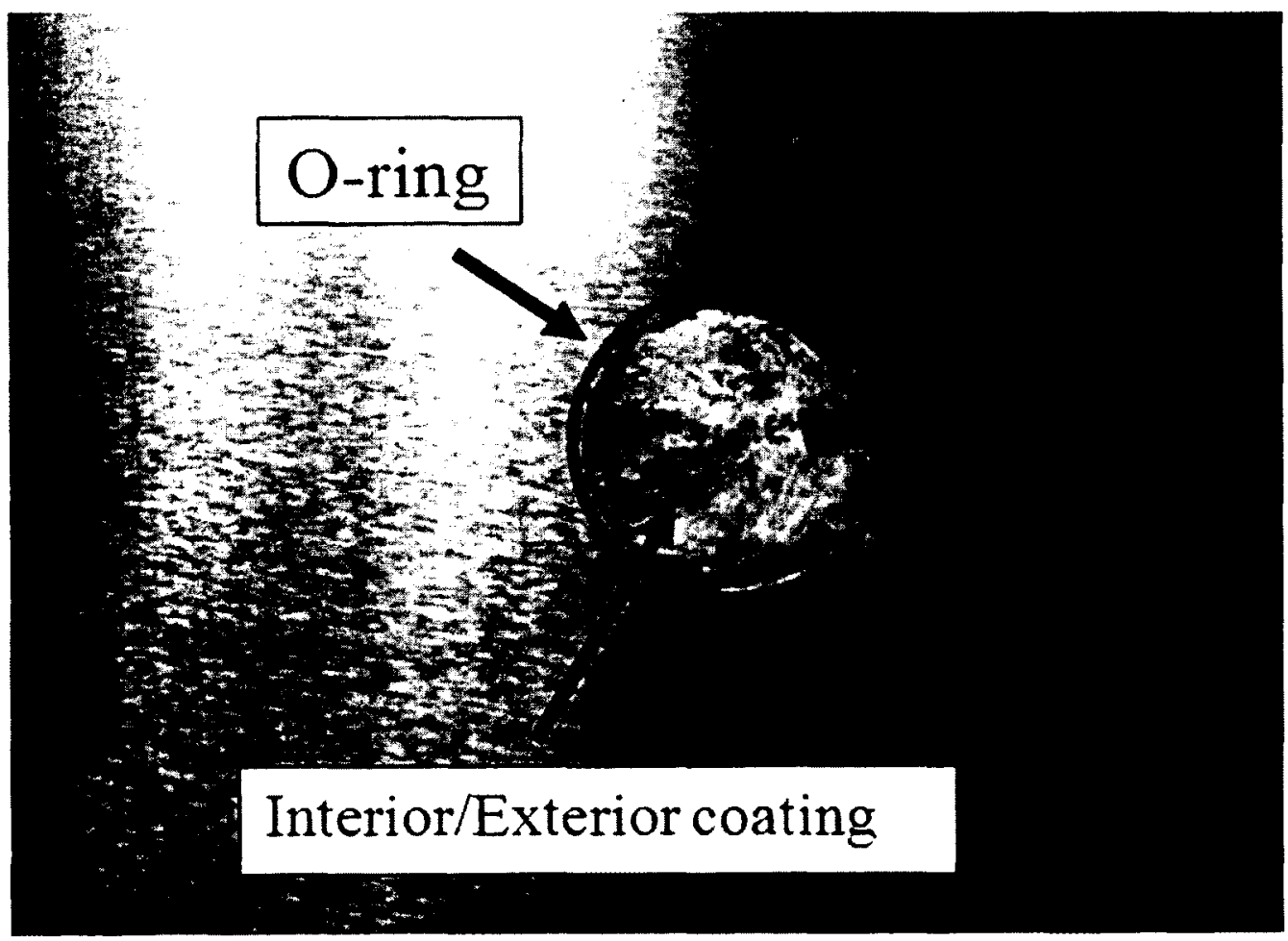

Figure 6-47 Condition of a coated aluminum sample after exposure to $1 \mathrm{M} \mathrm{NaCl}(\mathrm{pH}$ 8) for $\mathbf{2 5 0 0}$ hours in the horizontal cell (Figure 4-2). Initially the solution was deaerated with $N_{2}$ gas, then aerated after 1650 hours. Note that all the aluminum corroded away and only the coating on both sides was left. This is consistent with the build up of an acid solution between the two coating layers.

From this experiment, it can be seen that deaerating the electrolyte caused the corrosion process to stop and re-aerating the electrolyte caused corrosion. Referring to 
Figure 3-5, the passive zone where no corrosion is to occur is represented by lines 4-5. This means that the starting point, before the deaeration process, must be on lines $6-7$; above the pitting potential. Pitting has occurred in all EIS experiments with aerated coated aluminum samples, suggesting that in all cases the oxidizer level produced conditions in the samples above the pitting potential.

\subsection{4. $1 \mathrm{M} \mathrm{HCl}(\mathrm{pH}$ 0.1) Deaerated with Dry Ice}

As shown in section 6.3.2, samples in aerated acidic solutions start to fail only after a long exposure time. To test the samples in deaerated acid solutions, it was decided to start with a flawed sample, otherwise, the experiments might run for a long time. If the deaeration of acid was to have an effect on corrosion, then it was hoped that these effects would appear in the EIS data even for a flawed sample.

A $1 \mathrm{M} \mathrm{HCl}(\mathrm{pH} 0.1)$ solution was deaerated with dry ice and EIS data were collected as shown in Figure 6-48 and Figure 6-49. Since this sample was flawed to start with, as expected, the impedance measurements were low $\left(10^{5} \Omega\right.$ at $\left.0.01 \mathrm{~Hz}\right)$ and the phase plot is mostly resistive. Both plots showed spiking and positive phase angles were observed in the phase plot. These spikes are real, since the instrument is well within its comfortable measuring zone, and they arise due to corrosion currents.

After the deaeration of the solution, the impedance continued to drop and the capacitive to resistive transition frequency increased; the sample failed in a manner similar to samples exposed to aerated acid solutions. The EIS data for this sample demonstrate that deaeration of the acid solution had no measureable effect on the corrosion process once it was ongoing. No passivation or repassivation could occur in this strong acid solution. Once a flaw is there, there is no mechanism to stop corrosion. 


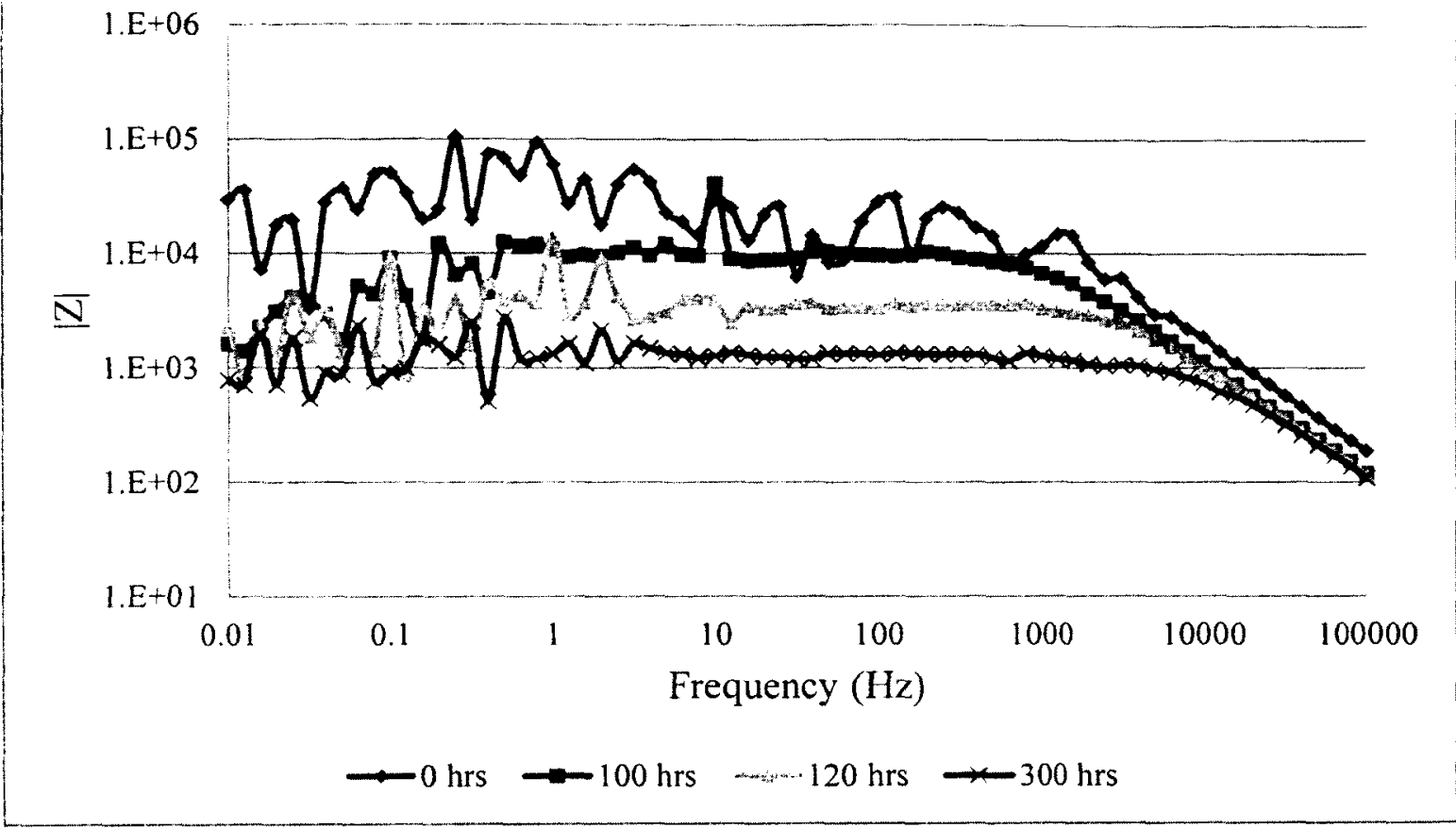

Figure 6-48 Impedance $(\Omega)$ measurements, using Solartron $1\left(\mathrm{VC}, 34.0 \pm 0.5 \mathrm{~cm}^{2}\right)$, for coated aluminum sample in deaerated $1 \mathrm{M} \mathrm{HCl}(\mathrm{pH} 0.1)$.

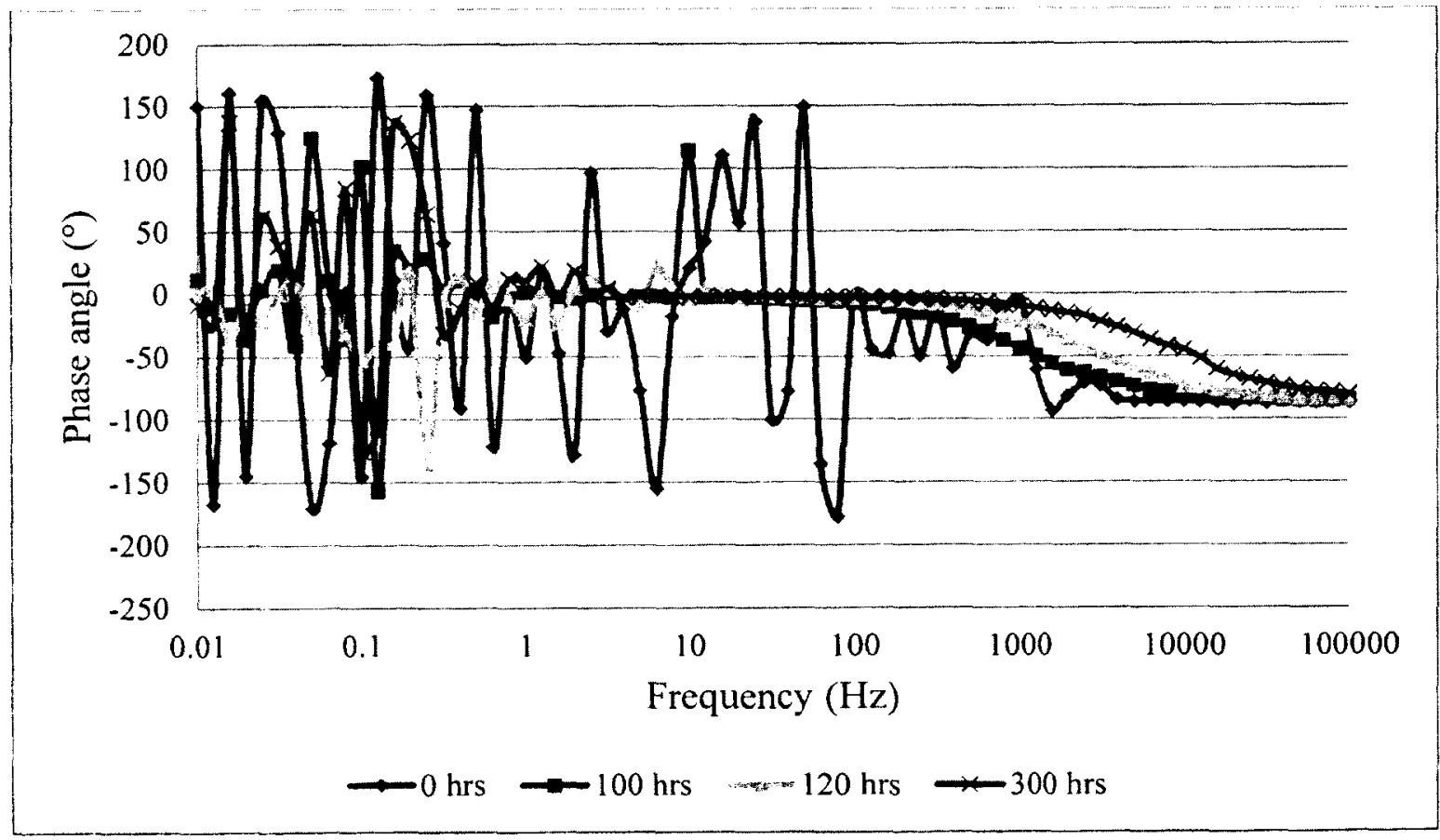

Figure 6-49 Phase angle measurements, using Solartron $1\left(\mathrm{VC}, 34.0 \pm 0.5 \mathrm{~cm}^{2}\right)$, for coated aluminum sample in deaerated $1 \mathrm{M} \mathrm{HCl}(\mathrm{pH} 0.1)$. 


\subsection{Experiments on Formed Can-Ends}

The study of can ends is in its preliminary stages. Attempts to study can ends using the available corrosion cells were unsuccessful due to configuration issues. Currently, modifications are being implemented into both corrosion cells so that can ends can be accommodated.

As explained in section 4.2 , can ends with two different coatings were available for testing. Samples of each were placed in a fume hood and filled with $1 \mathrm{M} \mathrm{HCl}(\mathrm{pH}$ 0.1). Discoloration, bubbles on the surface, and pitting were recorded. Data are summarized in Table 6-1.

Table 6-1 Observations from placing can ends in $1 \mathrm{M} \mathrm{HCl}$ solution

\begin{tabular}{|c|c|c|}
\hline \multirow{2}{*}{ Exposure in days } & \multicolumn{2}{|c|}{ Observations } \\
\cline { 2 - 3 } & Coating 1 & Coating 2 \\
\hline 0 & Samples placed in acid \\
\hline 3 & Bubbles on surface & Slight discoloration \\
\hline 8 & Pitting on surface & No change \\
\hline 10 & No change & Few bubbles on surface \\
\hline 17 & No change & Pitting on surface \\
\hline
\end{tabular}

Although it did not change colour, the sample with coating 1 was actively corroding after only 3 days, as evidenced by gas bubbles on the surface. It was readily apparent that all the corrosion sites were associated with the stamping on the can end (Figure 6-50). It appears that corrosion is more likely to occur at sites where the coating has been strained. 
Day 3

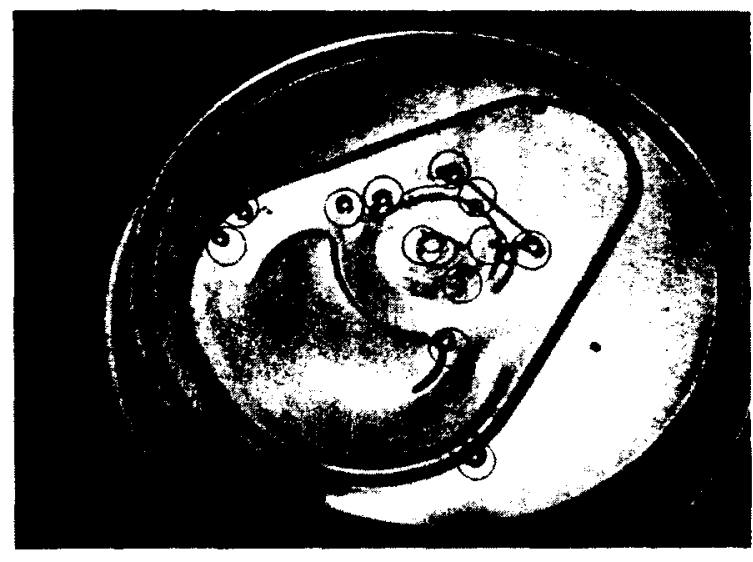

Day 8

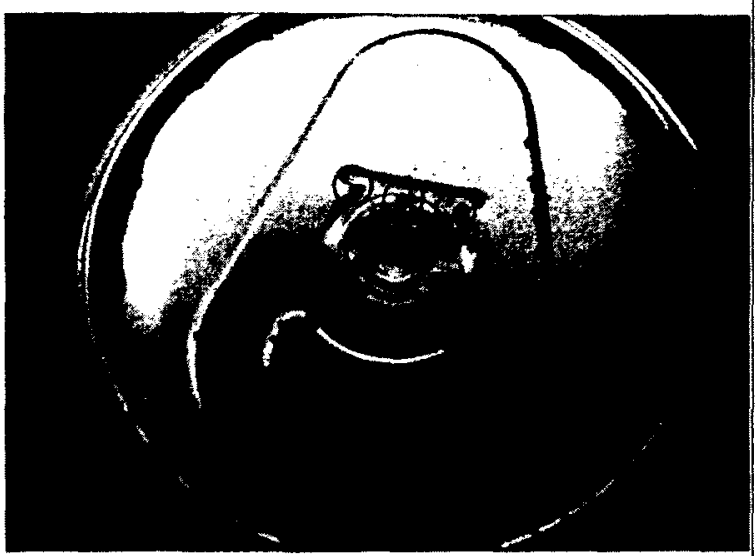

Figure 6-50 Sample with coating 1 showed the start of corrosion within 3 days. The corrosion sites, indicated by gas bubbles, are all in areas stressed by stamping of the can end.

On the other hand, the sample with coating 2 showed discoloration after 3 days. Bubbling on the surface was observed after 10 days and pitting was observed after 17 days. Once again, it was readily apparent that all the corrosion sites were associated with the stamping on the can end (Figure 6-51). It appears that corrosion is more likely to have happened at sites where the coating has been strained. 
Day 3

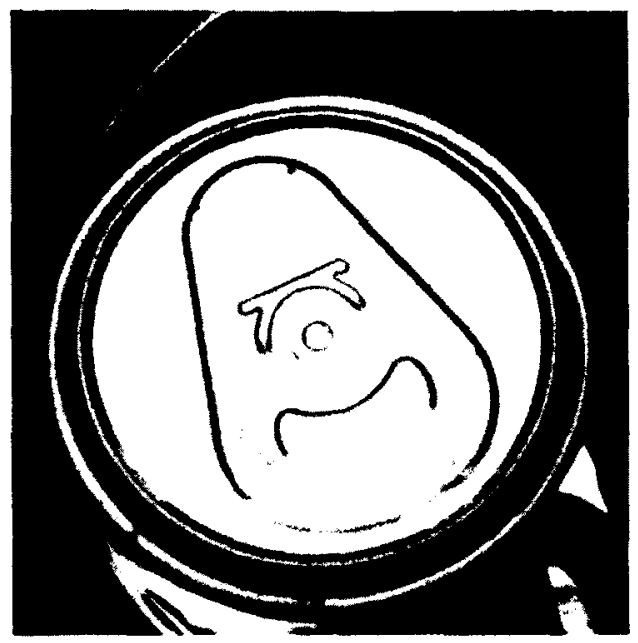

Day 17

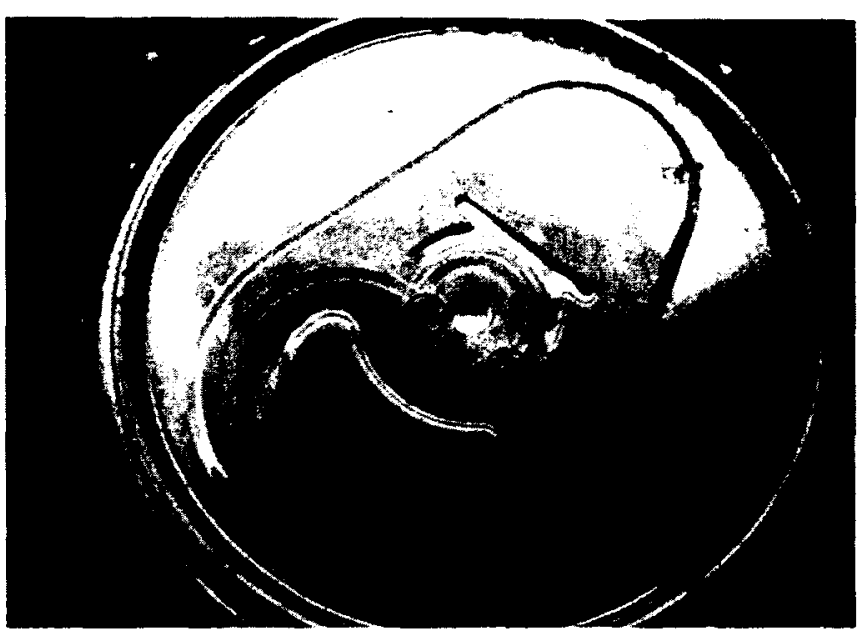

Figure 6-51 Sample with coating 2 showed discolouration after 3 days and pitting after 17 days.

\subsection{Conclusions}

\subsubsection{Conclusions from the Deliberate Flaws Experiments}

- An intact coated-aluminum sample can be identified by:

- Purely capacitive Bode and Nyquist plots in the frequency range of $10^{5} \mathrm{~Hz}-0.1 \mathrm{~Hz}$

- An impedance of $\geq 10^{8} \Omega$ at $0.1 \mathrm{~Hz}$;

- The measured current density in a potentiodynamic plot does not change with the applied voltage; the plot will produce a vertical line at $10^{-10} \mathrm{~A} / \mathrm{cm}^{2}-10^{-11} \mathrm{~A} / \mathrm{cm}^{2}$

- The Bode plot does not change when the electrolyte concentration is changed.

- A flawed coated-aluminum sample can be identified by: 
- The Bode plots are no longer purely capacitive, but are resistivecapacitive-resistive (high frequency $\rightarrow$ low frequency). Pseudo-inductive positive phase angles are observed at low frequencies $(<1 \mathrm{~Hz})$ in the phase plot, and loops with positive imaginary impedances are observed in the Nyquist plots;

- The impedance is smaller than an intact sample by orders of magnitude, being only $10^{4} \Omega-10^{6} \Omega$ at $0.1 \mathrm{~Hz}$;

- With increasing electrolyte concentration, the impedance drops, the magnitude of the low-frequency positive phase angle increases and the Nyquist curves decrease in magnitude;

- In a potentiodynamic plot, the measured current density is large (up to $1 \mathrm{~mA} / \mathrm{cm}^{2}$ ) and is strongly dependent on the applied voltage; corrosion can be clearly observed;

- As the DC voltage is increased, large and sudden increases in current demonstrate pitting corrosion; some pits are metastable and repassivate, while others are stable and do not repassivate;

- The size and direction (positive/negative) of the hysteresis loop in a potentiodynamic plot indicates the extent of corrosion occurring in the sample, and the ease of repassivation;

- At neutral $\mathrm{pH}$, samples attempted to repassivate and corrosion was slowed down by incomplete passivation. 


\subsubsection{Conclusions from Experiments in Aerated Solutions}

- For each solution, coated aluminum samples had similar failure times:

- Instantaneous for flawed samples;

- Up to two weeks for samples in neutral $\mathrm{pH}$;

Up to two months for samples in acidic solutions (the coatings were more resistant to acid).

- Indicators of corrosion were the same for all samples:

- Spiking at low frequencies;

- Drop in low frequency impedance $\left(10^{8} \Omega \rightarrow 10^{6} \Omega\right.$ at $\left.0.1 \mathrm{~Hz}\right)$;

- An increasing capacitive to resistive transition frequency;

- Inductive effects: positive phase angles in the phase plots at low frequencies and inductive loops in the Nyquist plots;

- Increase in the high-frequency $(>1 \mathrm{kHz})$ impedance attributed to stable oxide formation and loss of aluminum which alters the coating capacitance values (observed for some of the experiments);

- A downward shift in the impedance plot (observed for some of the experiments- see Figure 6-43 and Figure 6-45);

- Increased complexity of the Bode plots (new inflections and peaks observed for some of the experiments, see Figure 6-38 and Figure 6-39).

- EIS provided data that coincide with literature data:

- Some passivation occurred in neutral $\mathrm{pH}$ solutions (unstable oxide formed);

- No passivation occurred in low-pH solutions (no oxide formed); 
- Some passivation occurred and stable-oxide formed in mid-range $\mathrm{pH}$.

- DC polarization can be used to turn corrosion on and off; observed in sections 5.3.5 and 6.3.3;

- In service-condition $\mathrm{pH}$ solutions, spikes and positive phase angles still appeared but the drop in impedance is slower when compared to samples exposed to acidic and alkaline electrolytes; coatings are more resistant for service conditions;

- The presence of oxygen elevates the aluminum potential into the pitting region.

\subsubsection{Conclusions from Experiments in Deaerated Solutions}

- In neutral $\mathrm{pH}$, deaeration of the electrolyte completely prevents corrosion from occurring. After aeration of the solution, the samples rapidly failed;

- In deaerated solution, the aluminum is in the passive region below the pitting potential;

- Deaeration of aggressive acidic solutions ( $\mathrm{pH} 0.1$ ) does not hinder corrosion;

- No passive oxide forms in acidic solutions;

- With extended exposure, more indicators are observed in the Bode plots:

- Shift in the impedance plot (large drop after aeration of the solution);

- Occurrence of increased complexity (new inflections and peaks) in the Bode plots.

\subsubsection{Conclusions from Can-End Experiments}

- Corrosion sites were associated with the stamping on the can end;

- Corrosion is more likely to have happened at sites where the coating has been strained. 


\subsubsection{General Conclusions}

- Both EIS and potentiodynamic scans can be used to differentiate between an intact and a flawed sample;

- Both EIS and potentiodynamic scans can detect corrosion and its severity before it is visually observable;

- Initially unflawed samples last longer in acid solutions than in neutral solutions;

- Samples remain intact longer in deaerated solutions than aerated;

- There are clear signatures for corrosion in Bode and Nyquist plots. Sometimes these signatures are complex and cannot be quantitatively understood, but they do indicate corrosion of flawed samples;

- Several kinds of corrosion occur, uniform, stable and metastable pitting, and corrosion confined between coating layers. It appears that acid solutions occur locally between the coating layers when the aluminium is eaten away by corrosion in this confined space;

- Several parameters, such as the vertical shift in the impedance plot and increasing high-frequency impedance (Figure 6-38 and Figure 6-43) have not been fully explored. Further experimentation is required to fully understand the meaning of these occurrences;

- Indicators of corrosion were observed in the EIS data before they were visually observable on the surface of the samples. Spikes in the Bode plots and drops in impedance occurred before bubbles, oxide trees/clouds, or pits were visible in the corrosion cells; 
- With the use of EIS and potentiodynamic scans, together or separately, a specific testing procedure can be formulated to test coatings;

- The application of a positive DC voltage during an EIS scan makes the investigation more sensitive to defects. 


\section{Chapter 7. Conclusions and Recommendations}

\subsection{General}

The integrity of an aluminum beverage or food can depends on a polymeric coating (lacquer) that protects the can from a chemical environment containing highly aggressive solutions. Without this protective coating, cans would rapidly fail by corrosion within minutes. Epoxy resins are used as a protective coating in metal-based food and beverage cans. Bisphenol-A (BPA) is used as an intermediate in the production of epoxy resins. BPA can migrate from cans with epoxy coating into foods, especially at elevated temperatures (for example, for hot-fill or heat-processed canned foods).

BPA is one of the 23,000 chemical substances on the CEPA (Canadian Environmental Protection Act) Domestic Substance List (DSL) identified for further evaluation under government of Canada's Chemical Management Plan (CMP). Bisphenol-A, along with approximately 200 other chemicals, was identified as a high priority under the Government's Chemicals Management Plan; it was identified as a chemical that could affect reproduction. The risk assessment performed by Health Canada confirms that exposure levels are below those that could cause health effects, however, due to the uncertainty raised in some studies relating to the potential effects of low levels of Bisphenol-A, the Government of Canada is taking action to enhance the protection against BPA. Besides the effects of exposure to BPA, Environment Canada has identified concerns about the levels of Bisphenol-A that are being released to the environment. Thus, new regulations are currently being implemented and Health Canada's Food Directorate continues to place a high priority on the timely evaluation of pre-market submissions for BPA-free can coatings. This would mean that the coatings 
currently used to protect food and beverage must be replaced by new formulations. At present there is no fast and convenient method to ratify coatings before or during service, or to test new formulations.

This has initiated a need to develop, test and use new formulations of coatings/lacquers, which are as yet untested. The objective of this on-going research is primarily to develop detailed, standardized, accelerated-testing techniques and protocols using Electrochemical Impedance Spectroscopy (EIS), along with other electrochemical techniques, to evaluate the durability of Can End Stock (flat stock and converted ends).

EIS is a very powerful technique that can be used to study the structure of very high impedance coatings on materials, and monitor their degradation and failure. Cracks, holes, pores and blisters are all detectable with EIS from earlier in their history than by any non-electrochemical method. This method will enable evaluation of new coatings in a much shorter timeframe (weeks) than can currently be done with other techniques. The signatures in the EIS spectra that are quantitative indicators of coating failure and corrosion have been identified. A preliminary testing procedure has been developed and upon completion of future testing, robust laboratory procedures to perform these measurements will be finalized.

The main experimental method used in this research is EIS, complimented with Potentiodynamic scans. The research was carried out in three main stages. Stage 1, Preliminary Experiments (Chapter 5), was performed to determine if available EIS instruments are functional and produce consistent data. Samples included test circuits, fuel cells, kitchen aluminum foil, coated aluminum samples and a high-impedance epoxy 
polymer. Samples ranged from completely non-corrosive to highly corrosive. Signatures for corrosion and deteriorating coatings were recorded.

Stage 2 of the testing involved performing EIS experiments in aerated solutions (sections 6.2 and 6.3). The effects on the coated-aluminum samples of continuous exposure to different aggressive solutions, containing varied $\mathrm{pH}$ levels and salt concentrations were investigated. The signatures for corrosion were confirmed. These studies also established the background for the development of an accelerated testing procedure for aluminum lacquers.

Stage 3, the final stage, involved EIS experiments in de-aerated solutions (section 6.4) During Stage 1 and Stage 2 testing, samples failed in times varying from a few days to a few weeks, while in practice, in the field, similar cans last a very long time before they fail. Since in service conditions the coated-aluminum samples are exposed to deaerated environments, the effects of such conditions were explored to help understand the prolonged service life of aluminum beverage cans.

\subsection{Conclusions from Preliminary Experiments}

- Instrumentation Analysis: Two instruments were used for the gathering of EIS spectra: a Solartron 1260 impedance gain/phase analyzer (Solartron 1), and a Gamry PC4/300 (Gamry 1). Later in the investigation, a Solartron 1287 potentiostat with a Solartron 1255 gain/phase analyzer (Solartron 2) and a second Gamry PC4/300 (referred to as Gamry 2) were used for data comparison and verification, and to perform the DC experiments.

- All the instruments have varying operational limits, within which they all work well and provide consistent and reproducible results; 
- For the Gamry systems, it is very important to ensure that the cables used for the EIS measurements do not produce phantom peaks in the phase angle spectra. These peaks arise when the current-gain amplifier changes its range, and may be associated with inductance in the leads, because this sudden current change produces a marked positive spike in the phase angle;

- Gamry 1 and Gamry 2 are very well suited to measure EIS spectra for all kinds of samples over a wide range of frequencies; up to $10^{12} \Omega$ at $0.1 \mathrm{~Hz}$. Solartron 2 is capable of providing reliable results for impedances up to $10^{10} \Omega$ at $0.1 \mathrm{~Hz}$ while Solartron 1 is able to measure impedances up to $10^{8} \Omega$ at $0.1 \mathrm{~Hz}$;

- From the low impedance fuel cell experiments, it was determined once again that all the systems were reliable and provided essentially the same results.

\section{- Corrosion}

- Preliminary corrosion indicators were identified during the degradation of kitchen aluminum foil samples. It was observed that as increasing degradation and corrosion occurred with time, the capacitive-to-resistive transition frequency increased and there was a significant jump in the phase angle at low frequencies to positive values. These effects correlated with coating degradation;

- With the application of cathodic polarization, which is known to stop corrosion by dropping the potential below the corrosion potential into the immunity region, the inductive effects disappeared. This supports our assertion that inductive effects indicate corrosion; 
- In the EIS spectra, unexpected changes in the high frequency impedance were observed; the impedance slightly increased with exposure; this may be due to the disappearance of aluminum metal in between the layers of protective polymer;

- At neutral $\mathrm{pH}$ corroding aluminum readily passivates;

\section{- Sample surface area}

- By changing the sample surface area, the capacitance and impedance values change proportionally;

- Using a larger sample allows for better flaw detection which can be incorporated as a quality assurance technique;

- Using a smaller sample, the probability of the presence of surface defects is lower but this allows local testing of the coating quality;

\subsection{Conclusions from Experiments in Aerated Solutions}

- An intact coated-aluminum sample can be identified by:

- Purely capacitive Bode and Nyquist plots in the frequency range of $10^{5} \mathrm{~Hz}-0.1 \mathrm{~Hz}$;

- An impedance of $\geq 10^{8} \Omega$ at $0.1 \mathrm{~Hz}$;

- The measured current density in a potentiodynamic plot does not change with the applied voltage; the plot will produce a vertical line at $10^{-10} \mathrm{~A} / \mathrm{cm}^{2}-10^{-11} \mathrm{~A} / \mathrm{cm}^{2}$

- The Bode plot does not change when the electrolyte concentration is changed. 
- A flawed coated-aluminum sample can be identified by:

- The Bode plots are no longer purely capacitive, but are resistivecapacitive-resistive (high frequency $\rightarrow$ low frequency). Pseudo-inductive positive phase angles are observed at low frequencies $(<1 \mathrm{~Hz})$ in the phase plot, and loops with positive imaginary impedances are observed in the Nyquist plots;

- The impedance is smaller than an intact sample by orders of magnitude, being only $10^{4} \Omega-10^{6} \Omega$ at $0.1 \mathrm{~Hz}$;

- With increasing electrolyte concentration, the impedance drops, the magnitude of the low-frequency positive phase angle increases and the Nyquist curves decrease in magnitude;

- In a potentiodynamic plot, the measured current density is large (up to $1 \mathrm{~mA} / \mathrm{cm}^{2}$ ) and is strongly dependent on the applied voltage; corrosion can be clearly observed;

- As the DC voltage is increased, large and sudden increases in current demonstrate pitting corrosion; some pits are metastable and repassivate, while others are stable and do not repassivate;

- The size and direction (positive/negative) of the hysteresis loop in a potentiodynamic plot indicates the extent of corrosion occurring in the sample, and the ease of repassivation;

- At neutral $\mathrm{pH}$, samples attempted to repassivate and corrosion was slowed down by incomplete passivation. 
- For each solution, coated aluminum samples had similar failure times:

- Instantaneous for flawed samples;

- Up to two weeks for samples in neutral $\mathrm{pH}$;

- Up to two months for samples in acidic solutions (the coatings were more resistant to acid).

- Indicators of corrosion were the same for all samples:

- Spiking at low frequencies;

- Drop in low frequency impedance $\left(10^{8} \Omega \rightarrow 10^{6} \Omega\right.$ at $\left.0.1 \mathrm{~Hz}\right)$;

- An increasing capacitive to resistive transition frequency;

- Inductive effects: positive phase angles in the phase plots at low frequencies and inductive loops in the Nyquist plots;

- Increase in the high-frequency $(>1 \mathrm{kHz})$ impedance attributed to stable oxide formation and loss of aluminum which alters the coating capacitance values (observed for some of the experiments);

- A downward shift in the impedance plot (observed for some of the experiments- see Figure 6-43 and Figure 6-45);

- Increased complexity of the Bode plots (new inflections and peaks observed for some of the experiments, see Figure 6-38 and Figure 6-39).

- EIS provided data that coincide with literature data:

- Some passivation occurred in neutral $\mathrm{pH}$ solutions (unstable oxide formed);

- No passivation occurred in low-pH solutions (no oxide formed);

- Some passivation occurred and stable-oxide formed in mid-range $\mathrm{pH}$. 
- DC polarization can be used to turn corrosion on and off; observed in sections 5.3.5 and 6.3.3;

- In service-condition pH solutions, spikes and positive phase angles still appeared but the drop in impedance is slower when compared to samples exposed to acidic and alkaline electrolytes; coatings are more resistant for service conditions;

- The presence of oxygen elevates the aluminum potential into the pitting region.

\subsection{Conclusions from Can-End Experiments}

- Corrosion sites were associated with the stamping on the can end;

- Corrosion is more likely to have happened at sites where the coating has been strained.

\subsection{Conclusions from Experiments in Deaerated Solutions}

- In neutral $\mathrm{pH}$, deaeration of the electrolyte completely prevents corrosion from occurring. After aeration of the solution, the samples rapidly failed;

- In deaerated solution, the aluminum is in the passive region below the pitting potential;

- Deaeration of aggressive acidic solutions ( $\mathrm{pH} 0.1$ ) does not hinder corrosion;

- No passive oxide forms in acidic solutions;

- With extended exposure, more indicators are observed in the Bode plots:

- Shift in the impedance plot (large drop after aeration of the solution);

- Occurrence of increased complexity (new inflections and peaks) in the Bode plots. 


\subsection{General Conclusions}

- EIS can be utilized as a tool for fast indication of a protective coating versus a flawed coating. It can be used as a quality assurance test.

- A protective coating will have a purely capacitive Bode plot and no changes in current density with the applied voltage in a potentiodynamic plot;

- A flawed coating will have a resistive-capacitive-resistive Bode plot and in a potentiodynamic scan will have large changes in current with the applied voltage. A totally penetrated coating will have a low impedance resistive Bode plot;

- By changing the electrolyte concentration, large changes in the Bode plot will be observed for a flawed coating, while for an intact coating, no changes will be observed.

- EIS can be used to detect degradation/corrosion before it is visually observable. Changes in EIS data are observed long before any indications are observed in the corrosion cell. EIS corrosion indicators include:

- Spikes in impedance and phase plots;

- An increasing capacitive to resistive transition frequency;

- Inductive effects such as positive phase angles;

- Transitions in the impedance plot (generally downwards);

- An increase in the high frequency impedance;

- Increased complexity in the Bode plots; 
- Running EIS and polarization simultaneously has shown that spikes in impedance and phase-angles plus positive phase angles occur when a sample is corroding. Cathodic polarization turns corrosion off, and when it was applied, the impedance increased, while spiking and positive phase angles were all diminished, implying that these effects are related to corrosion;

- Drops in impedance only occurred after spiking had been observed;

- Increases in impedance (passivation) were observed after the spikes disappeared. It seems that spikes correlate with the formation of flaws (pits) and once these pits are blocked due to the oxide (actually observed during experiments), the impedance increased once again.

- Using a single parameter, such as low frequency impedance, to rank coatings is not very practical

- In the passivation region, EIS data has shown that the impedance periodically falls and rises with time, probably due to formation of oxides on the metal surface. The formed oxide blocks the pore/flaws/pits and stops conduction between the electrolyte and the metal substrate;

- Both drops and rises in low-frequency impedance can happen when chemistry occurs at the metal/oxide/solution interface, therefore lowfrequency impedance measurements can be confounded by chemistry, meaning that individual measurements may not be good indicators of the coating quality; 
- Using the low frequency impedance as an indicator is not practical due to oxide formation. The value of low frequency impedance at one particular time is not indicative of the protective quality of the coating but rather the availability of a conduction path between the electrolyte and the metal substrate.

- The testing conditions greatly affect the performance of the coated aluminum samples. During testing, some samples failed in as little as a few days, while others lasted several months.

- Samples lasted longer in acid solutions than in neutral solutions, contrary to expectations, because protective aluminium oxide does not form at low $\mathrm{pH}$;

- Samples last much longer in deaerated neutral solutions than in aerated solutions;

- The time to failure of the samples depended on:

- the $\mathrm{pH}$;

- presence of oxygen;

- Concentration of $\mathrm{Cl}^{-}$ions.

- EIS can provide an insight on the type of corrosion occurring in the sample; different signatures can be observed for different types of corrosion

- The sudden appearance of inductive effects and spiking in the Bode plots indicate the formation of pitting in the sample. Depending on the testing conditions, oxide might form on the surface and the metastable pits become clogged. On the other hand, if a compact oxide layer is unable to 
form, the metastable pits become stable pits after which large drops are observed in the Bode impedance plots.

\subsection{Recommended Testing Procedure}

The following conditions are recommended to be used for testing new coatings:

- Potentiostat: The potentiostat with the highest measuring capabilities is the Gamry PC4/300. At the same time, a Solartron 1287 potentiostat with a Solartron 1255 gain/phase analyzer can also be used. However, a standalone Solartron 1260 will have noise in the Bode plots, at the low frequencies, for high impedance samples and therefore not recommended for use;

- Corrosion cell: Either the vertical or the horizontal cell can be used for performing EIS experiments. One advantage of the vertical cell is the visibility of the sample under test. Also one is more likely to locate defects, because it uses large samples. However, depending on the quality of the coating, it is sometimes difficult to find a defect-free sample for use with this cell. Since samples in the horizontal cell are much smaller, compared to the vertical cell, finding a defect-free sample is an easier task. A big disadvantage is that the surface of the corroding sample cannot readily be seen.

- Testing Conditions: The aim of this testing procedure is to test several coatings in the shortest time possible. This can be achieved by:

○ using a solution of aerated $1 \mathrm{M} \mathrm{NaCl}(\mathrm{pH} 8)$ as the electrolyte;

- collecting EIS data in the frequency range of $10^{-1} \mathrm{~Hz}-10^{5} \mathrm{~Hz}$;

- collecting EIS data with an AC excitation of $20 \mathrm{mV}$ and $1 \mathrm{~V}$ DC; 


\subsection{Recommendations for Future Work}

- Several observation, such as the downward/upward shift in impedance Bode plot (see Figure 6-43 and Figure 6-45), increasing impedances at high frequencies (see Figure 6-38) and increasing complexity in the phase plots (see Figure 6-39) have not been fully explored. It is not known whether, with extended exposure, the solutions affect the coating thickness or not. Further experimentation is required to understand fully the meaning of these occurrences. Several samples can be exposed to the different solutions under different conditions, and thickness and mass measurements can be taken periodically;

- During this research, samples were tested in acidic $\mathrm{pH}$, neutral $\mathrm{pH}$ and midrange $\mathrm{pH}$. Experiments in alkaline solutions might reveal information that was not previously observed;

- With some modifications, the corrosion cells can accommodate formed can-ends, and experiments can be repeated to observe the performance of the final product as opposed to the performance of the coated aluminum sheet. 


\section{Bibliography}

[1] Alcoa, "How aluminum cans are made," [Online]. Available: http://www.alcoa.com/rigid_packaging/en/about/making_cans.asp. [Accessed 20 Sep 2012].

[2] Health Canada, "Update on the Food Directorate's Risk Management Commitments for Bisphenol A as a Part of Canada's Chemicals Management Plan," [Online]. Available: http://www.hc-sc.gc.ca/fn-an/securit/packag-emball/bpa/bpa rm-gr-2012-09-eng.php. [Accessed 20 September 2012].

[3] Environment Canada, "Notice requiring the preparation and implementation of pollution prevention plans with respect to Bisphenol-A in industrial effluents," Canada Gazette, vol. 146, p. 139, 2012.

[4] Gamry Instruments, "A snapshot of electrochemical impedance spectroscopy," Application Note, 2011.

[5] D. Loveday, P. Peterson and B. Rodgers, "Evaluation of Organic Coatings with Electrochemical Impedance Spectroscopy Part 1: Fundamentals of Electrochemical Impedance Spectroscopy," Gamry Instrument, 2004.

[6] Gamry Instruments, "Two-, three-, and four-electrode experiments," Application Note, 2011.

[7] J. R. Macdonald, Impedance spectroscopy: Emphasizing solid materials and systems, Chapel Hill, NC: John Wiley \& Sons, 1987.

[8] Gamry Instruments, "Basics of electrochemical impedance spectroscopy," Application Note, 2010.

[9] E. Cano, D. Lafuente and D. M. Bastidas, "Use of EIS for the evaluation of the protective properties of coatings for metallic cultural heritage: a review," J. Solid State Electrochem, vol. 14, pp. 381-391, 2010.

[10] A. S. Khanna, High-performance organic coatings, Woodhead Publishing, 2008.

[11] F. D. P. L. Bonora and L. Fedrizzi, "Electrochemical impedance spectroscopy as a tool for invesitgating underpaint corrosion," Electrochimica Acta, vol. 41, pp. 1073-1082, 1996.

[12] D. Loveday, P. Peterson and B. Rodgers, "Evaluation of Organic Coatings with Electrochemical Impedance Spectroscopy Part 2: Application of EIS to coatings," Gamry Instruments, 2004.

[13] Gamry Instruments, "EIS of organic coatings and paints," Application Note, 2011.

[14] R. W. Revie and H. H. Uhlig, Corrosion and Corrosion Control, Hoboken New Jersey : John Wiley \& Sons, 2008.

[15] R. W. Revie, Uhlig's Corrosion Handbook, Pennington New Jersey: John Wiley \& Sons, 2000.

[16] P. Marcus, Corrosion Mechanisms in theory and practice, Boca Raton, FL: Taylor \& Francis group, 2012.

[17] Argentum Solutions, "Pitting Corrosion," 2012. [Online]. Available:

http://www.argentumsolutions.com/wiki/en/Pitting_Corrosion\#General_Mechanism_Information. [Accessed August 2012]. 
[18] A. Augustyniak and W. Ming, "Early detection of aluminum corrosion via turn-on fluorescence in smart coatings," Progress in organic coatings, vol. 71, pp. 406-412, 2011.

[19] M. G. Fontana, Corrosion Engineering, Ohio: McGraw-Hill Inc., 1986.

[20] G. McRae, Lecture Notes: MAAE 4906C-Corrosion and corrosion control, Ottawa, ON, 2012.

[21] "Corrosion Anodic protection," [Online]. Available: http://events.nace.org/library/corrosion/AnodProtect/passivecurve.asp. [Accessed July 2012].

[22] Z. Szklarska-Smialowska, "Pitting corrosion of aluminum," Corrosion science, vol. 41, pp. 1743. $1767,1999$.

[23] R. Baboian, Corrosion Tests and standards: Application and interpretation, Baltimore MD: ASTM, 2005.

[24] M. Poelman, M. Olivier, N. Gayarre and J. Petitjean, "Electrochemical study of different ageing tests for the evaluation of a cataphoretic epoxy primer on aluminium," Progress in Organic coatings, vol. 54, pp. 55-62, 2005.

[25] R.G. Hu, S. Zhang, J.F. Bu, C.J. Lin and G.L. Song, "Recent progress in corrosion protection of magnesium alloys by organic coatings," Progress in Organic coatings, vol. 73, pp. 129-141, 2012 .

[26] F. Mansfeld, "A correlation between salt spray and electrochemical impedance spectroscopy test results for conversion-coated aluminum alloys," Corrosion science, vol. 54, pp. 595-597, 1998.

[27] G. Bierwagen, C. Jeffcoate, J. Li, S. Balbyshev, D. Tallman and D. Mills, "The use of electrochemical noise methods (ENM) to study thick, high impedance coatings," Progress in Organic coatings, vol. 29, pp. 21-29, 1996.

[28] S. Skale, V. Dolecek and M. Slemnik, "Substitution of the constant phase element by Warburg impedance for protective coatings," Corrosion Science, vol. 49, pp. 1045-1055, 2007.

[29] D. D. MacDonald, "Reflections on the history of electrochemical impedance spectroscopy," Electrochimica Acta, vol. 51, pp. 1376-1388, 2006.

[30] B. R. Hinderliter, S. G. Croll, D. E. Tallman, Q. S. Q and G. P. Bierwagen, "Interpretation of EIS data from accelerated exposure of coated metals based on modeling of coating physical properties," Electrochimica Acta, vol. 51, pp. 4505-4515, 2006.

[31] F. Gui and R. Kelly, "Performance assessment and prediction of corrosion prevention compounds with electrochemical impedance spectroscopy," Corrosion science, vol. 61, pp. 119-129, 2005.

[32] E. V. Westing, G. Ferrari and J. d. Wit, "The determination of coating performance with impedance measurements - I. Coating polymer properties," Corrosion science, vol. 34, pp. 1511 $1530,1993$.

[33] G. Bierwagen, D. Tallman, J. Li, L. He and C. Jeffcoate, "EIS studies of coated metals in accelerated exposure," Progess in Organic coatings, vol. 46, pp. 148-157, 2003.

[34] F. L. Floyd, S. Avudaiappan, J. Gibson, B. Mehta, P. Smith, T. Provderd and J. Escarsega, "Using electrochemical impedance spectroscopy to predict the corrosion resistance of unexposed coated metal panels," Progress in Organic coatings, vol. 66, pp. 8-34, 2009. 
[35] J. N. Murray, "Electrochemical test methods for evaluating organic coatings on metals: an update. Part I. Introduction and generalities regarding electrochemical testing of organic coatings," Progress in Organic Coatings, vol. 30, pp. 225-233, 1997.

[36] F. Mansfeld, "Use of electrochemical impedance spectroscopy for the study of corrosion protection by polymer coatings," Journal of applied electrochemistry, vol. 25, pp. 187-202, 1995.

[37] J. N. Murray, "Electrochemical test methods for evaluating organic coatings on metals: an update. Part 1II: Mutliple test parameter measurements," Progress in Organic Coatings, vol. 31, pp. 375$390,1997$.

[38] U. Rammelt and G. Reinhard, "Application of electrochemical impedance spectroscopy (EIS) for characterizing the corrosion-protective performance of organic coatings on metals," Progress in Organic Coatings, vol. 21, pp. 205-226, 1992.

[39] B. S. Skerry and D. Eden, "Electrochemical testing to assess corrosion protective coatings," Progress in Organic Coatings, vol. 15, pp. 269-285, 1987.

[40] J.T. Zhang, J.-M. Hu, J.Q. Zhang and C.N. Cao, "Studies of water transport behavior and impedance models of epoxy coated metals in $\mathrm{NaCl}$ solution by EIS," Progress in Organic Coatings, vol. 51, pp. 145-151, 2004.

[41] M. Zheludkevich, K. Yasakau, A. Bastos, O. Karavai and M. Ferreira, "On the application of electrochemical impedance spectroscopy to study the self-healing properties of protective coatings," Electrochemistry communications, vol. 9, pp. 2622-2628, 2007.

[42] C. Chiavari, A. Colledan, A. Frignani and G. Brunoro, "Corrosion evaluation of traditional and new bronzes for artistic castings," Materials Chemistry and Physics, vol. 95, pp. 252-259, 2006.

[43] C. Chiavari, K. Rahmouni, H. Takenouti, S. Joiret and P. V. P. e. al, "Composition and electrochemical properties of natural patinas of outdoor bronze monuments," Electrochimica Acta, vol. 52, pp. 7760-7769, 2007.

[44] M. Bernard, E. Dauvergne, M. Evesque, M. Keddam and H. Takenouti, "Reduction of silver tarnishing and protection against subsequent corrosion," Corrosion Science, vol. 47, pp. 663-679, 2005.

[45] M. Evesque, M. Keddam and H. Takenouti, "The formation of self assembling membrane of hexadecane-thiol on silver to prevent the tarnishing," Electrochimica Acta, vol. 49, pp. 29372943, 2004.

[46] F. Bovard, T. Burleigh and A. Smith, "Electrochemical Impedance spectroscopy of electrocoated aluminium food cans," Electrochimica Acta, vol. 40, pp. 201-207, 1995.

[47] J. Grandle and S. Taylor, "Electrochemical Impedance Spectroscopy of Coated Aluminum Beverage Containers: Part 1- Determination of an optimal parameter for large sample evaluation," Corrosion Science, vol. 50, pp. 792-803, 1994.

[48] J. Grandle and S. Taylor, "Electrochemical Impedance Spectroscopy of Coated Aluminum Beverage Containers: Part 2- Statistical analysis of performance," Corrosion Science, vol. 53, pp. 347-355, 1997. 
[49] M. Stratmann, A. Leng, W. Furbeth, H. Streckel, H. Gehmecke and K. G. Brinkhaus, "The scanning Kelvin probe; a new technique for the in situ analysis of the delamination of organic coatings," Progress in Organic Coatings, vol. 27, pp. 261-267, 1996.

[50] R. Souto, B. Normand, H. Takenouti and M. Keddam, "Self-healing processes in coil-coated cladding studied by the scanning vibrating electrode," Electrochimica Acta, vol. 55, pp. 4551$4557,2010$.

[51] G. Baril, C. Blanc, M. Keddam and N. Pébère, "Local electrochemical impedance spectroscopy applied to the corrosion behavior of an AZ91 magnesium alloy," J.Electrochem. Soc., vol. 150, pp. B488-493, 2003.

[52] A. Mierisch and S. Taylor, "Understanding the degradation of organic coatings using local electrochemical impedance methods," J. Electrochem. Soc., vol. 150, p. B303, 2003.

[53] L. Philippe, G. Walter and S. Lyon, "Investigating localized degradation of organic coatings," $J$. Electrochem. Soc., vol. 150, p. B111, 2003.

[54] C. Zhong, X. Tang and Y. Cheng, "Corrosion of steel under the defected coating studied by localized electrochemical impedance spectroscopy," Electrochimica Acta, vol. 53, pp. 4740-4747, 2008.

[55] V. M. Huanga, S.-L. Wu, M. E. Orazem, N. Pébère, B. Tribollet and V. Viviera, "Local electrochemical impedance spectroscopy: A review and some recent developments," Electrochimica Acta, vol. 56, pp. 8048-8057, 2011.

[56] P. L. Cabot, J. A. Garrido, E. Perez and J. Virgili, "High Potential Cathodic Polarization of Oxidized Aluminium Electrodes in Neutral and Acid Media," Corrosion Science, vol. 26, p. 357-369, 1986.

[57] S. Analytical, 1260 Impedance/Gain-Phase Analyzer Operating Manual, Hampshire: Solartron, 1996.

[58] Gamry Instruments, "EIS Measurement of a Very Low Impedance Lithium Ion Battery," Application note, 2011.

[59] Gamry Instruments, PC4 Potentiostat/Galvanostat/ZRA Operator's manual, USA: Gamry Instruments, 1999.

[60] "Tait Cell," Princeton Applied Research, 2010. [Online]. Available: http://www.princetonappliedresearch.com/Our-Products/Accessories/Tait-Cell-Kit.aspx. [Accessed 12 August 2012].

[61] "Flat Cell," Princeton Applied Research, 2010. [Online]. Available: http://www.princetonappliedresearch.com/Our-Products/Accessories/Flat-Cell-Kit.aspx. [Accessed 15 August 2012].

[62] D. Chan, D. Ouellette, E. Matida, C. Cruickshank and G. McRae, "Electrochemical impedance spectroscopy study on a flowing electrolyte-direct methanol fuel cell," in 11th International Conference on Sustainable Energy Technologies (SET-2012), Vancouver, Canada, 2012.

[63] F. Farelas, M. Galicia, B. Brown, S. Nesic and H. Castaneda, "Evolution of dissolution processes at the interface of carbon steel corroding in a $\mathrm{CO} 2$ environment studied by EIS," Corrosion Science, vol. 52, pp. 509-517, 2010. 
[64] Y. Xin, C. Liu, W. Zhang, J. Jiang, G. Tang, X. Tian and P. K. Chu, "Electrochemical Behavior A12O3/Al Coated Surgical AZ91 Magnesium Alloy in Simulated Body Fluids," Journal of The Electrochemical Society, vol. 155, pp. C178-C182, 2008.

[65] B. A. Boukamp, "Interpretation of an 'inductive loop' in the impedance of an oxygen ion conducting electrolyte/metal electrode system," Solid State Ionics, vol. 143, pp. 47-55, 2001.

[66] D. Z. Ahmad, "The kinetics of anodic and cathodic polarization of aluminium and its alloys," Anti-Corrosion Methods and Materials, vol. 33, pp. 4-11, 1986.

[67] K. Nisancioglu, "Cathodic polarization of commercially pure aluminium," Corrosion Science, vol. 19 , no. 8, pp. 537-552, 1979.

[68] S. Moon and S. Pyun, "The corrosion of pure aluminium during cathodic polarization in aqueous solutions," Corrosion Science, vol. 39, pp. 399-408, 1997.

[69] M. Darabara, L. Bourithis, S. Zinelis and G. D. Papadimitriou, "Susceptibility to localized corrosion of stainless steel and NiTi endodontic instruments in irrigating solutions," International Endodontic Journal, vol. 37, pp. 705-710, 2004.

[70] B. Wilde, "A Critical Appraisal of Some Popular Laboratory Electrochemical Tests for Predicting the Localized Corrosion Resistance of Stainless Alloys in Seawater," Corrosion, vol. 28, p. 283, 1972.

[71] D. C. Silverman, "Tutorial On Polexpert And The Cyclic Potentiodynamic Polarization Technique," [Online]. Available: http://www.argentumsolutions.com/tutorials/polexpert_tutorialpg5.html. [Accessed September 2012]. 